\title{
Preparative Fluorous Mixture Synthesis of Diazonium-Functionalized Oligo(Phenylene Vinylene)s
}

\author{
Huahua Jian and James M. Tour* \\ Department of Chemistry, Department of Mechanical Engineering and \\ Materials Science and \\ Center for Nanoscale Science and Technology, MS-222, Rice University, \\ 6100 South Main Street, \\ Houston, Texas 77005 \\ tour@rice.edu \\ Supporting Information
}

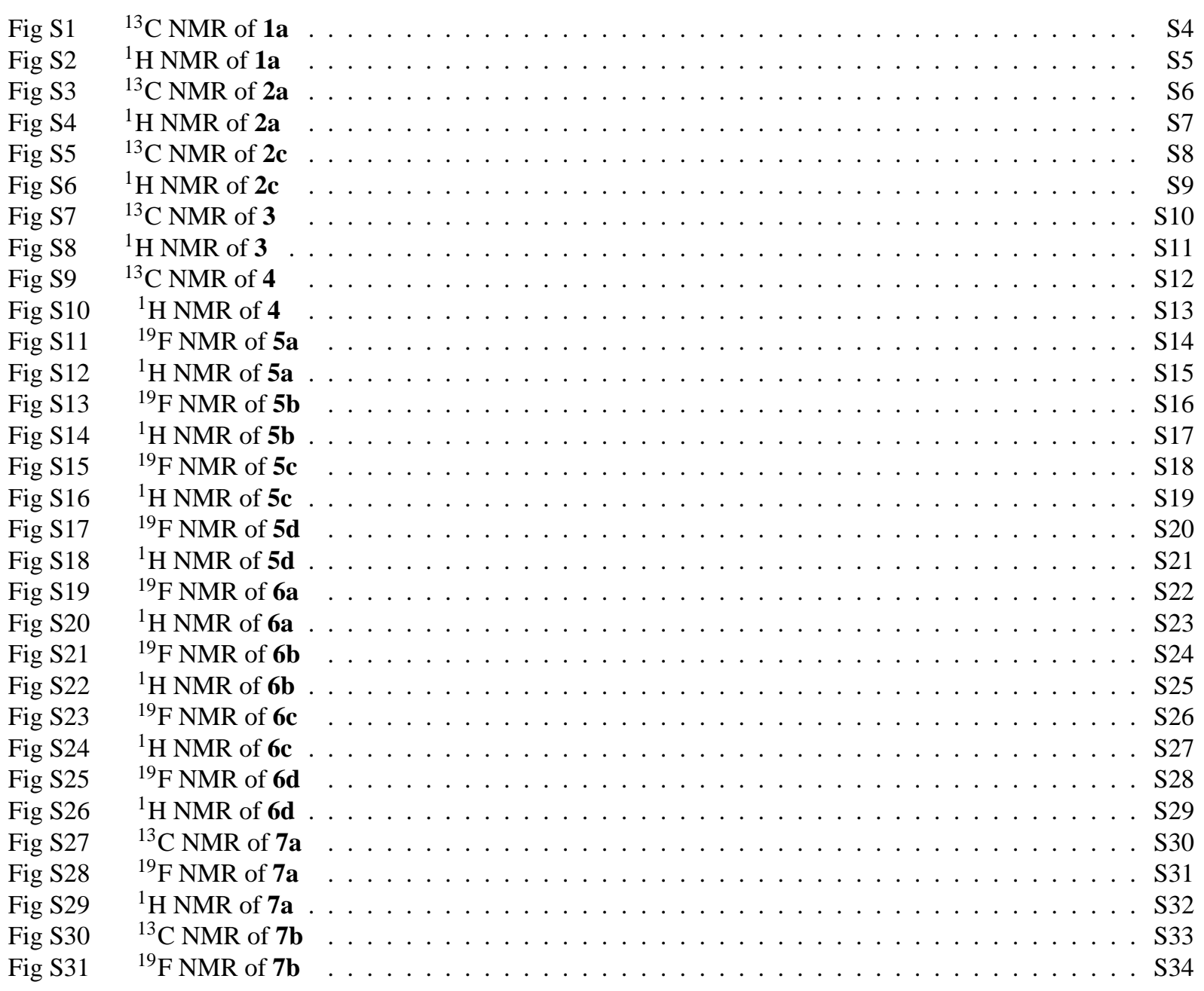


Fig S32

Fig S33

Fig S34

Fig S35

Fig S36

Fig S37

Fig S38

Fig S39

Fig S40

Fig S41

Fig S42

Fig S43

Fig S44

Fig S45

Fig S46

Fig S47

Fig S48

Fig S49

Fig S50

Fig S51

Fig S52

Fig S53

Fig S54

Fig S55

Fig S56

Fig S57

Fig S58

Fig S59

Fig S60

Fig S61

Fig S62

Fig S63

Fig S64

Fig S65

Fig S66

Fig S67

Fig S68

Fig S69

Fig S70

Fig S71

Fig S72

Fig S73

Fig S74

Fig S75

Fig S76

Fig S77

Fig S78

Fig S79

Fig S80

Fig S81

Fig S82

Fig S83

Fig S84
${ }^{1} \mathrm{H}$ NMR of $\mathbf{7 b}$

${ }^{13} \mathrm{C}$ NMR of $7 \mathrm{c}$

${ }^{19} \mathrm{~F}$ NMR of $7 \mathbf{c}$

${ }^{1} \mathrm{H}$ NMR of $7 \mathbf{c}$

${ }^{13} \mathrm{C}$ NMR of $7 d$

${ }^{19} \mathrm{~F}$ NMR of $7 d$

${ }^{1} \mathrm{H}$ NMR of 7d

${ }^{13} \mathrm{C}$ NMR of $\mathbf{8 a}$

${ }^{19}$ F NMR of $8 \mathbf{a}$

${ }^{1} \mathrm{H}$ NMR of $8 \mathbf{a}$

${ }^{13} \mathrm{C}$ NMR of $\mathbf{8 b}$

${ }^{19} \mathrm{~F}$ NMR of $\mathbf{8 b}$

${ }^{1} \mathrm{H}$ NMR of $\mathbf{8 b}$

${ }^{13} \mathrm{C}$ NMR of $8 \mathrm{c}$

${ }^{19} \mathrm{~F}$ NMR of $\mathbf{8 c}$

${ }^{1} \mathrm{H}$ NMR of $8 \mathrm{c}$

${ }^{13} \mathrm{C}$ NMR of $8 d$

${ }^{19} \mathrm{~F}$ NMR of $8 \mathbf{d}$

${ }^{1} \mathrm{H}$ NMR of $8 d$

${ }^{13} \mathrm{C}$ NMR of $\mathbf{1 1}$

${ }^{1} \mathrm{H}$ NMR of 11

${ }^{13} \mathrm{C}$ NMR of $\mathbf{1 2}$

${ }^{1} \mathrm{H}$ NMR of 12

${ }^{13} \mathrm{C}$ NMR of $\mathbf{1 5}$

${ }^{1} \mathrm{H}$ NMR of $\mathbf{1 5}$

${ }^{13} \mathrm{C}$ NMR of 16

${ }^{1} \mathrm{H}$ NMR of 16

${ }^{13} \mathrm{C}$ NMR of 18

${ }^{1} \mathrm{H}$ NMR of $\mathbf{1 8}$

${ }^{13} \mathrm{C}$ NMR of 19

${ }^{1} \mathrm{H}$ NMR of 19

${ }^{13} \mathrm{C}$ NMR of 22

${ }^{1} \mathrm{H}$ NMR of 22

${ }^{13} \mathrm{C}$ NMR of 23

${ }^{1} \mathrm{H}$ NMR of 23

${ }^{13} \mathrm{C}$ NMR of 24

${ }^{1} \mathrm{H}$ NMR of $\mathbf{2 4}$

${ }^{13} \mathrm{C}$ NMR of $\mathbf{2 5}$

${ }^{1} \mathrm{H}$ NMR of 25

${ }^{13} \mathrm{C}$ NMR of $\mathbf{2 6}$

${ }^{1} \mathrm{H}$ NMR of 26

${ }^{13} \mathrm{C}$ NMR of 27

${ }^{1} \mathrm{H}$ NMR of 27

${ }^{13} \mathrm{C}$ NMR of 28

${ }^{1} \mathrm{H}$ NMR of 28

${ }^{1} \mathrm{H}$ NMR of $\mathbf{3 2}$

${ }^{13} \mathrm{C}$ NMR of $\mathbf{3 3}$

${ }^{1} \mathrm{H}$ NMR of $\mathbf{3 3}$

${ }^{13} \mathrm{C}$ NMR of $\mathbf{3 4}$

${ }^{1} \mathrm{H}$ NMR of 34

${ }^{13} \mathrm{C}$ NMR of $\mathbf{3 5 a}$

${ }^{1} \mathrm{H}$ NMR of 35a

${ }^{13} \mathrm{C}$ NMR of $35 \mathrm{c}$ 
Fig S85

Fig S86

Fig S87

Fig S88

Fig S89

Fig S90

Fig S91

Fig S92

Fig S93

Fig S94

Fig S95

Fig S96

Fig S97

Fig S98

Fig S99

Fig S100

Fig S101

Fig S102

Fig S103

Fig S104

Fig S105

Fig S106

Fig S107

Fig S108

Fig S109

Fig S110

Fig S111

Fig S112

Fig S113

Fig S114

Fig S115

Fig S116

Fig S117

Fig S118

Fig S119

Fig S120

Fig S121

Fig S122

Fig S123

Fig S124

Fig S125

Fig S126

Fig S127

Fig S128

Fig S129
${ }^{1} \mathrm{H}$ NMR of $\mathbf{3 5 c}$

${ }^{13} \mathrm{C}$ NMR of $\mathbf{3 5 d}$

${ }^{1} \mathrm{H}$ NMR of $\mathbf{3 5 d}$

${ }^{13} \mathrm{C}$ NMR of $\mathbf{3 6}$

${ }^{1} \mathrm{H}$ NMR of 36

${ }^{13} \mathrm{C}$ NMR of 38

${ }^{1} \mathrm{H}$ NMR of 38

${ }^{13} \mathrm{C}$ NMR of 39

${ }^{1} \mathrm{H}$ NMR of 39

${ }^{13} \mathrm{C}$ NMR of $\mathbf{4 0}$

${ }^{1} \mathrm{H}$ NMR of 40

${ }^{13} \mathrm{C}$ NMR of $\mathbf{4 1}$

${ }^{1} \mathrm{H}$ NMR of 41

${ }^{1} \mathrm{H}$ NMR of $\mathbf{4 2}$

${ }^{13} \mathrm{C}$ NMR of 43

${ }^{1} \mathrm{H}$ NMR of $\mathbf{4 3}$

${ }^{13} \mathrm{C}$ NMR of $\mathbf{4 4}$

${ }^{1} \mathrm{H}$ NMR of 44

${ }^{13} \mathrm{C}$ NMR of $\mathbf{4 5}$

${ }^{1} \mathrm{H}$ NMR of 45

${ }^{13} \mathrm{C}$ NMR of 46

${ }^{1} \mathrm{H}$ NMR of 46

${ }^{13} \mathrm{C}$ NMR of $\mathbf{4 7}$

${ }^{1} \mathrm{H}$ NMR of 47

${ }^{13} \mathrm{C}$ NMR of 48

${ }^{1} \mathrm{H}$ NMR of 48

${ }^{1} \mathrm{H}$ NMR of $49 \mathrm{~b}$

${ }^{19}$ F NMR of $\mathbf{5 1 b}$

${ }^{1} \mathrm{H}$ NMR of $\mathbf{5 1 b}$

${ }^{1} \mathrm{H}$ NMR of 51c

${ }^{19} \mathrm{~F}$ NMR of 51d

${ }^{1} \mathrm{H}$ NMR of 51d

${ }^{1} \mathrm{H}$ NMR of $\mathbf{5 2 a}$

${ }^{1} \mathrm{H}$ NMR of $\mathbf{5 2 b}$

${ }^{1} \mathrm{H}$ NMR of $\mathbf{5 2 c}$

${ }^{19} \mathrm{~F}$ NMR of $\mathbf{5 2 d}$

${ }^{1} \mathrm{H}$ NMR of $\mathbf{5 2 d}$

${ }^{19} \mathrm{~F}$ NMR of $\mathbf{5 4 b}$

${ }^{1} \mathrm{H}$ NMR of 54b

${ }^{19} \mathrm{~F}$ NMR of $\mathbf{5 4 c}$

${ }^{1} \mathrm{H}$ NMR of $\mathbf{5 4 c}$

${ }^{19} \mathrm{~F}$ NMR of 59d

${ }^{1} \mathrm{H}$ NMR of 59d

${ }^{13} \mathrm{C}$ NMR of $62 \mathrm{~b}$

${ }^{1} \mathrm{H}$ NMR of $\mathbf{6 2 b}$
S88

S89

S90

S91

S92

S93

S94

S95

S96

S97

S98

S99

S100

S101

S102

S103

S104

S105

S106

S107

S108

S109

S110

S111

S112

S113

S114

S115

S116

S117

S118

S119

S120

S121

S122

S123

S124

S125

S126

S127

S128

S129

S130

S131

S132 

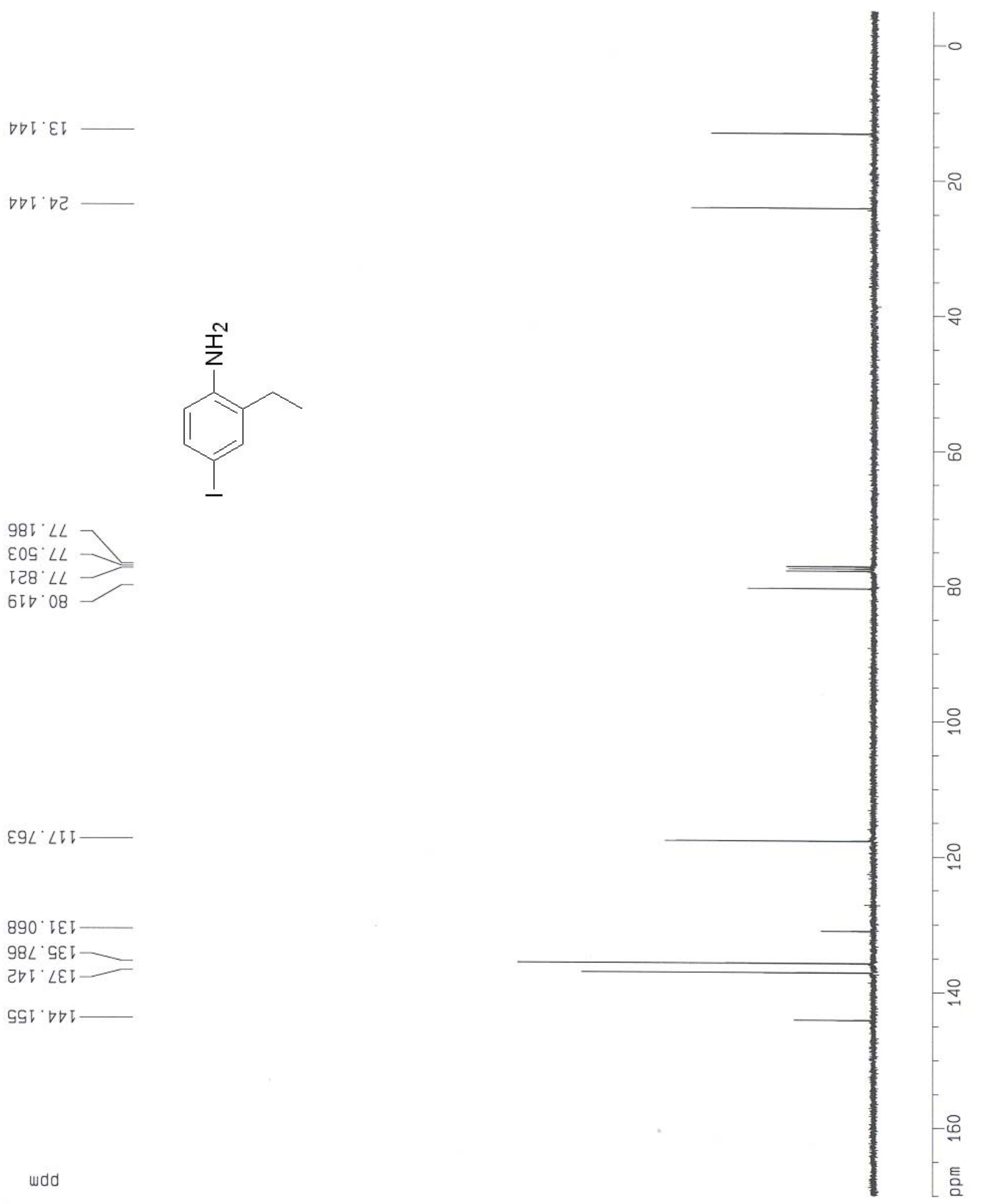

Figure S1: ${ }^{13} \mathrm{C}$ NMR of 1a 


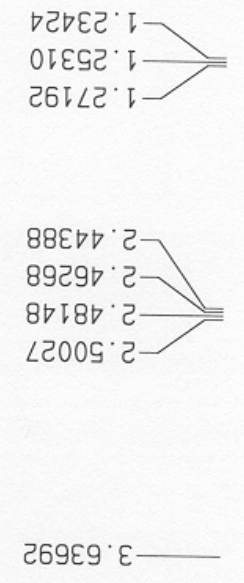

ट69ع9 $\varepsilon$

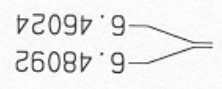

$\angle 080 \varepsilon^{\circ} \angle-$

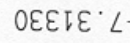
ट६८८ह ' $L$ 06દદє ' $\angle$ 8ट0 LE $L$

wdd

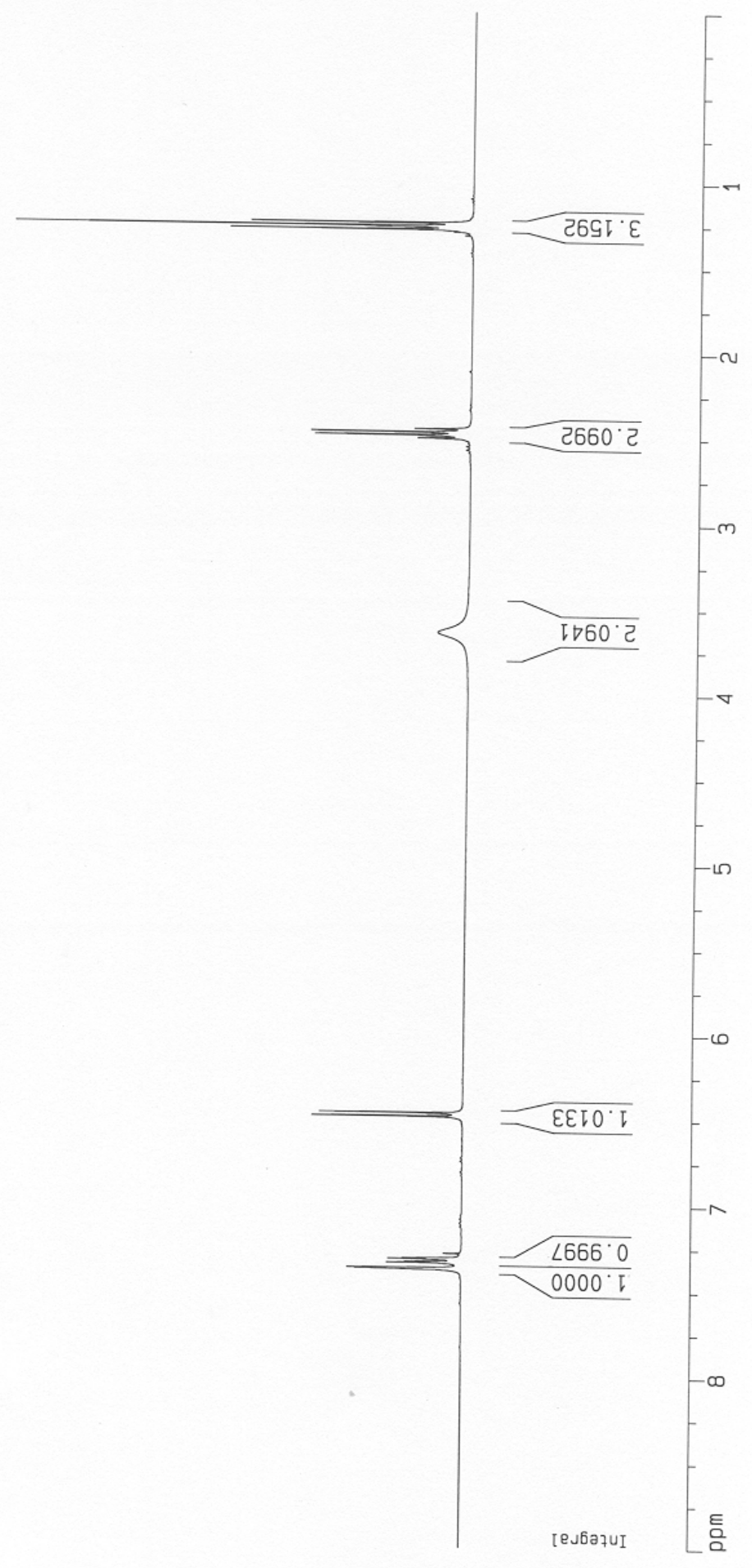

Figure S2: ${ }^{1} \mathrm{H}$ NMR of 1a 


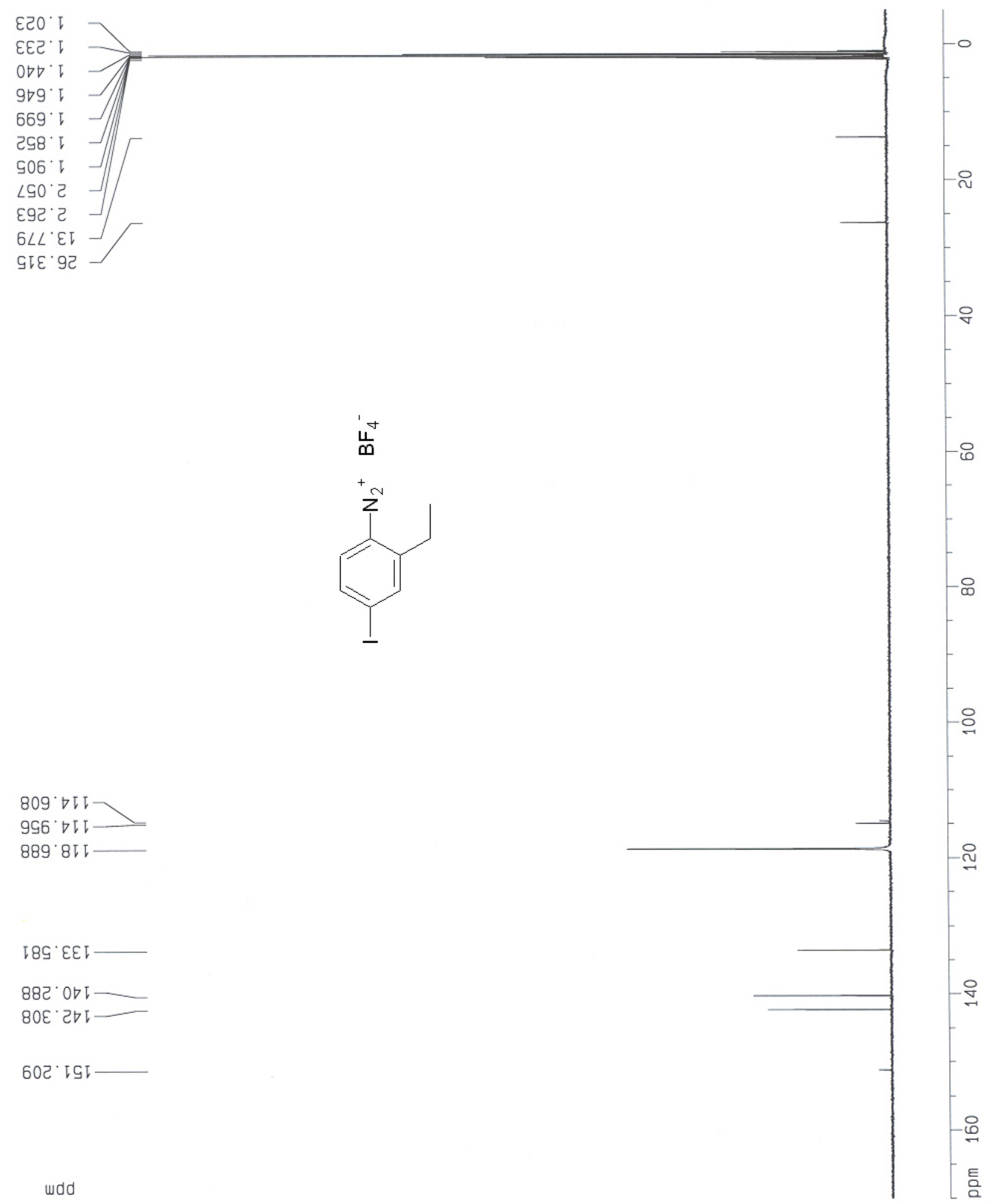

Figure S3: ${ }^{13} \mathrm{C}$ NMR of $\mathbf{2 a}$ 

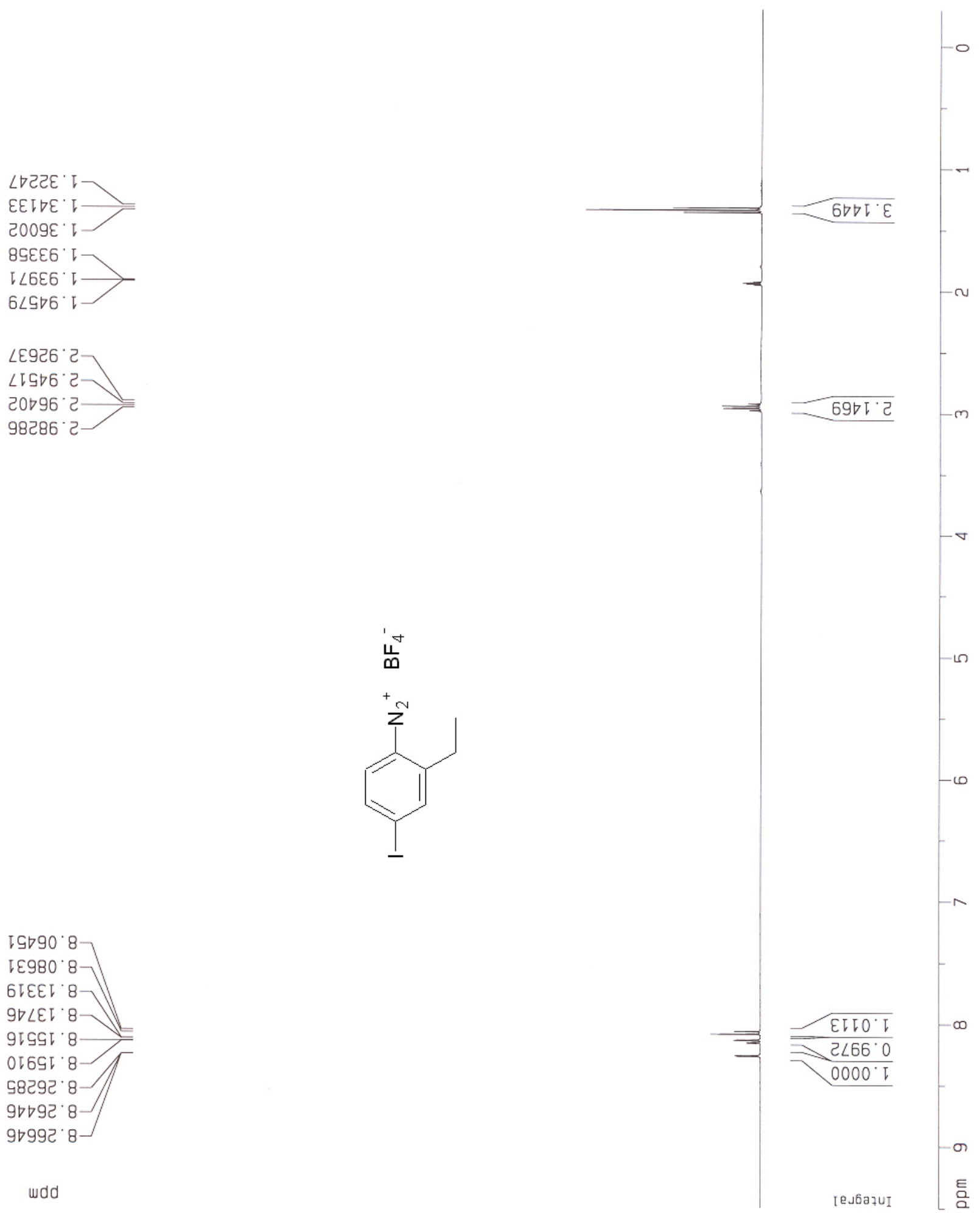

Figure S4: ${ }^{1} \mathrm{H}$ NMR of $\mathbf{2 a}$ 


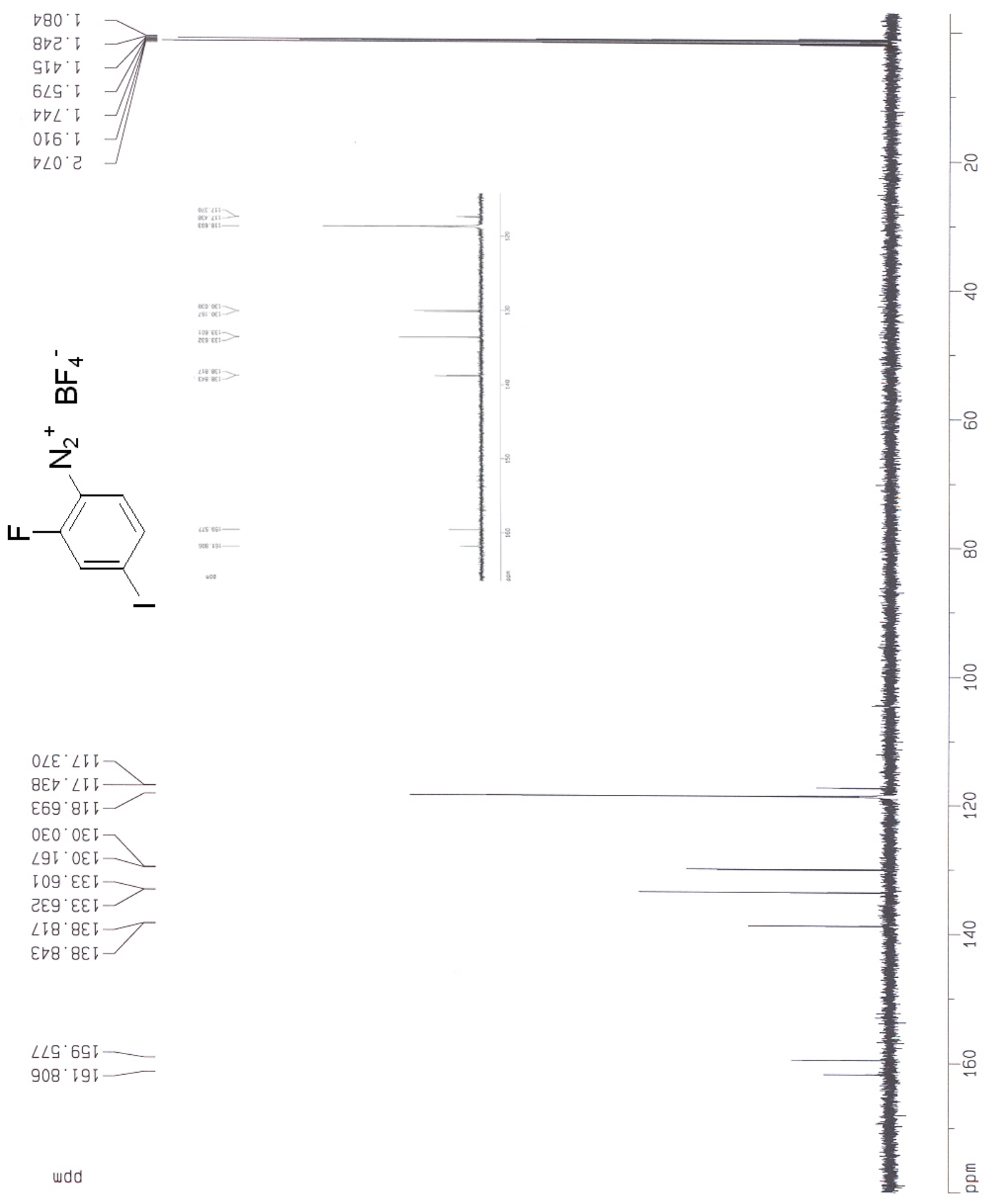

Figure S5: ${ }^{13} \mathrm{C}$ NMR of $\mathbf{2 c}$ 

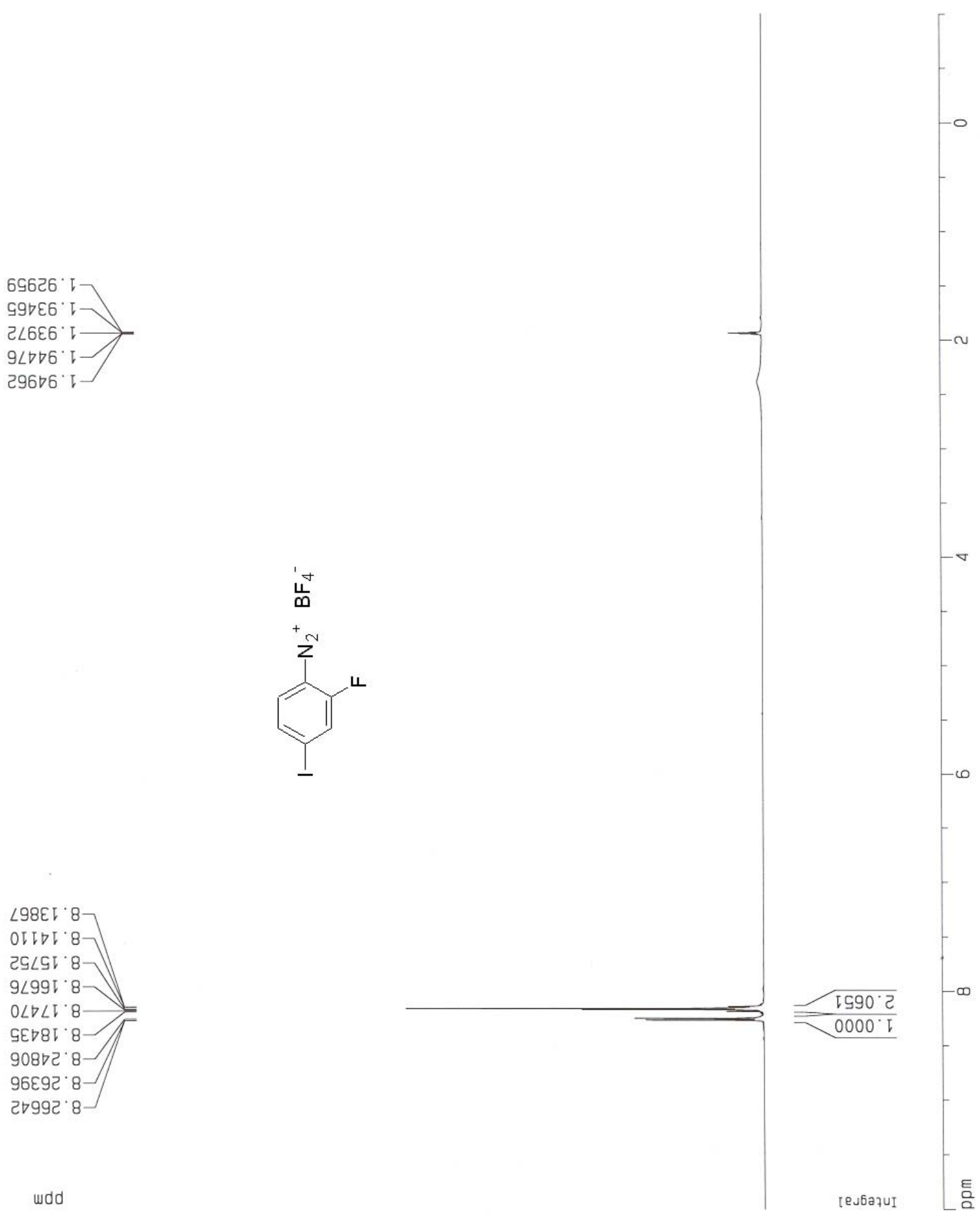

Figure S6: ${ }^{1} \mathrm{H}$ NMR of $\mathbf{2 c}$ 
८6Г टा

$0 ટ 9^{\circ} \varepsilon 己$

8IL'IS

$\varepsilon ट L^{\circ} \varepsilon \subseteq$

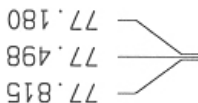

$0 \angle 6^{\circ} 021$

$\angle O C^{\circ} O E I-$

$808^{\circ}$ LEI-

$\angle 60^{\circ} O D I$

wdd

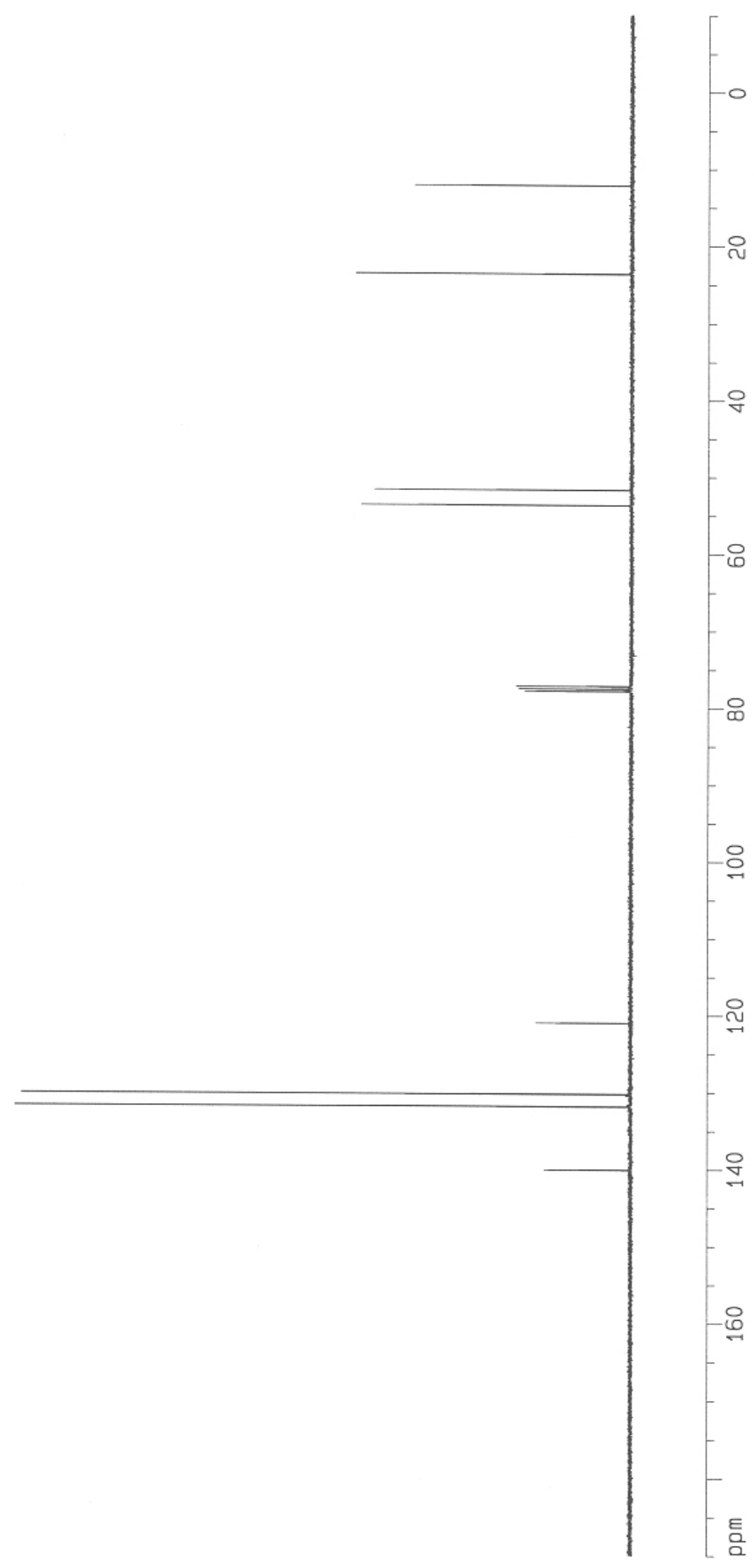

Figure S7: ${ }^{13} \mathrm{C}$ NMR of 3 

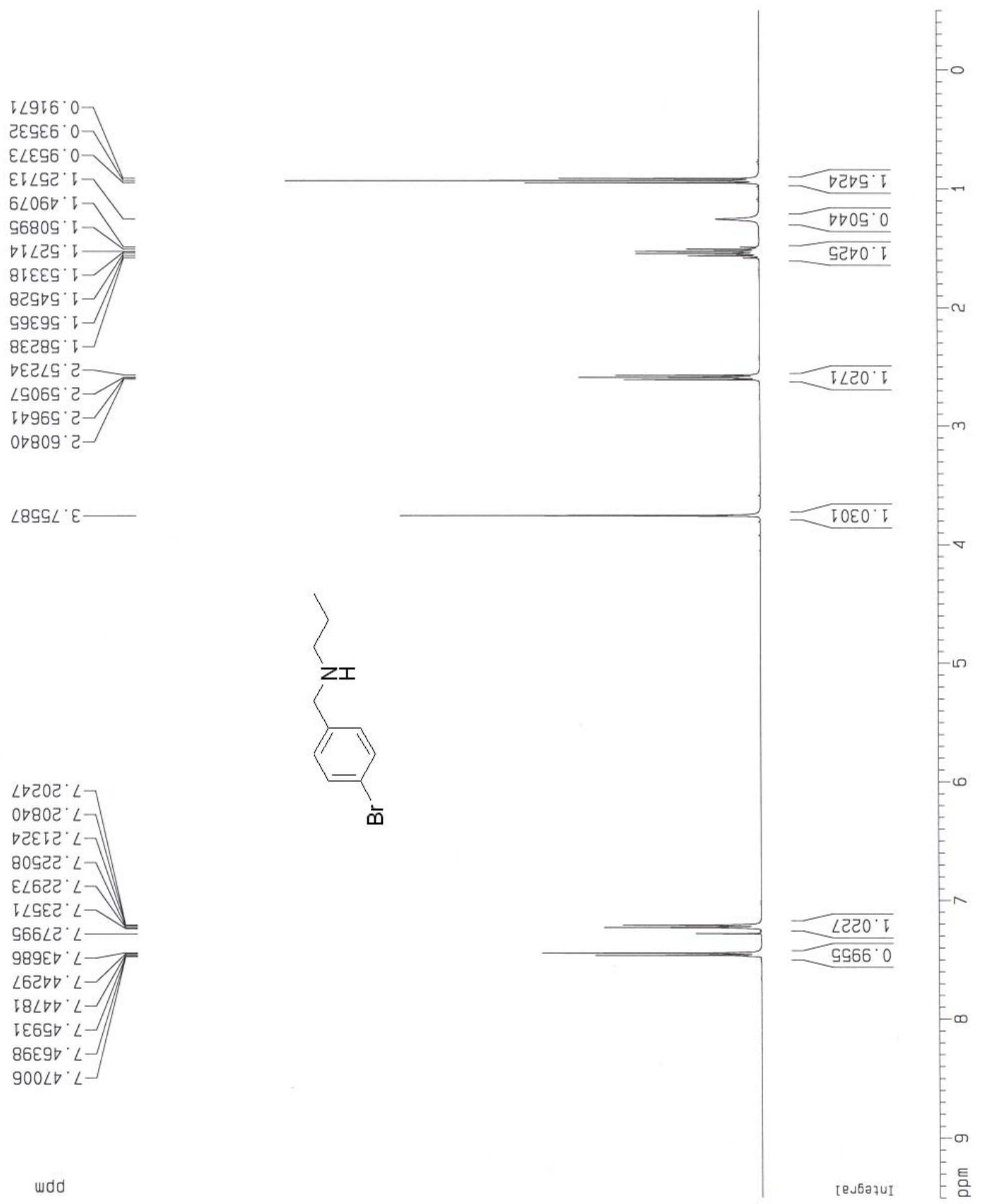

$\angle \Delta C O C^{\circ} \angle-$ $0 \rightarrow 80 C^{\circ} L$

DटEIट. $L$ 809टर $\angle-$ $\varepsilon \angle G \angle 2 \cdot \angle>$ I GEट $\angle$ G66 $\angle 2 \cdot \angle$ 989Eम $\angle \longrightarrow$ $\angle 6 ट \nabla \nabla D^{\circ} \angle-$ I8LDV $\angle-$ IE69० $\angle-$ $86 \varepsilon 9 \nabla^{\circ} \mathrm{L}-$ $900 \angle \nabla^{\circ} \angle J$

wdd

Figure S8: ${ }^{1} \mathrm{H}$ NMR of $\mathbf{3}$ 


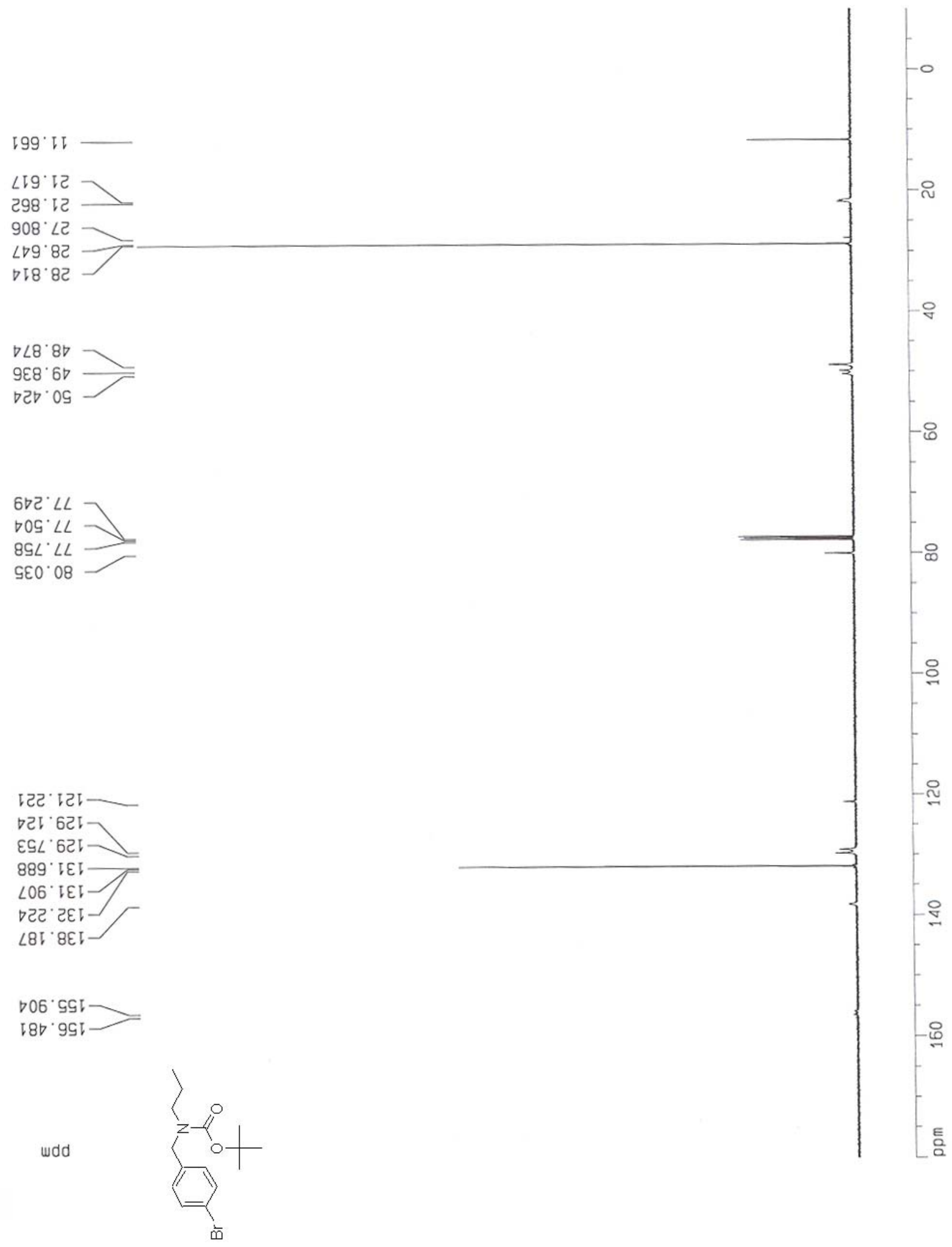

Figure S9: ${ }^{13} \mathrm{C}$ NMR of $\mathbf{4}$ 


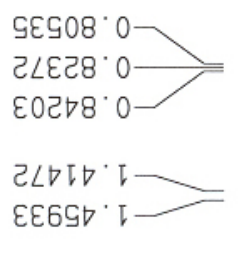

$60890^{\circ} \varepsilon \sim$

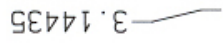

$\angle \nabla \neg E^{\circ} \nabla$

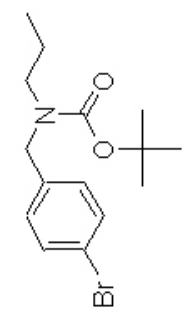

$8 \nabla 980^{\circ} \mathrm{L}$

$\varepsilon \angle 6 \angle C^{\prime} \angle \longrightarrow$

6L88E $\angle \longrightarrow$

9960 ' $L$

wdd

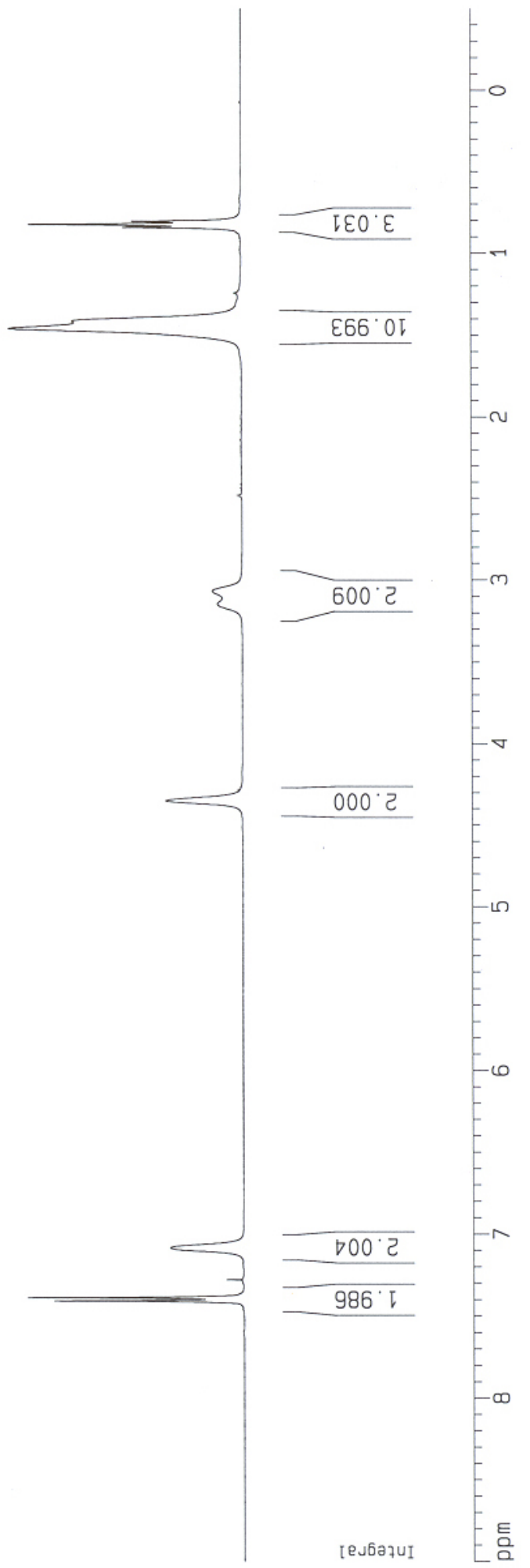

Figure S10: ${ }^{1} \mathrm{H}$ NMR of 4 


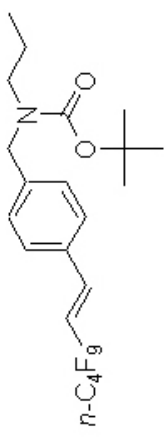

ટદ 9ટાoE. $951-$ $\angle ट \cdot 921-1$ $\angle 9^{\circ} \nabla ट$ -

จ ${ }^{\circ}$ 扎i $\cdot \tau \tau$ i $->$

$99^{\circ}$ น8$99 \cdot 18-$ $79^{\circ}$ โ8$79^{\circ}$ น ह9. โ8-

wdd

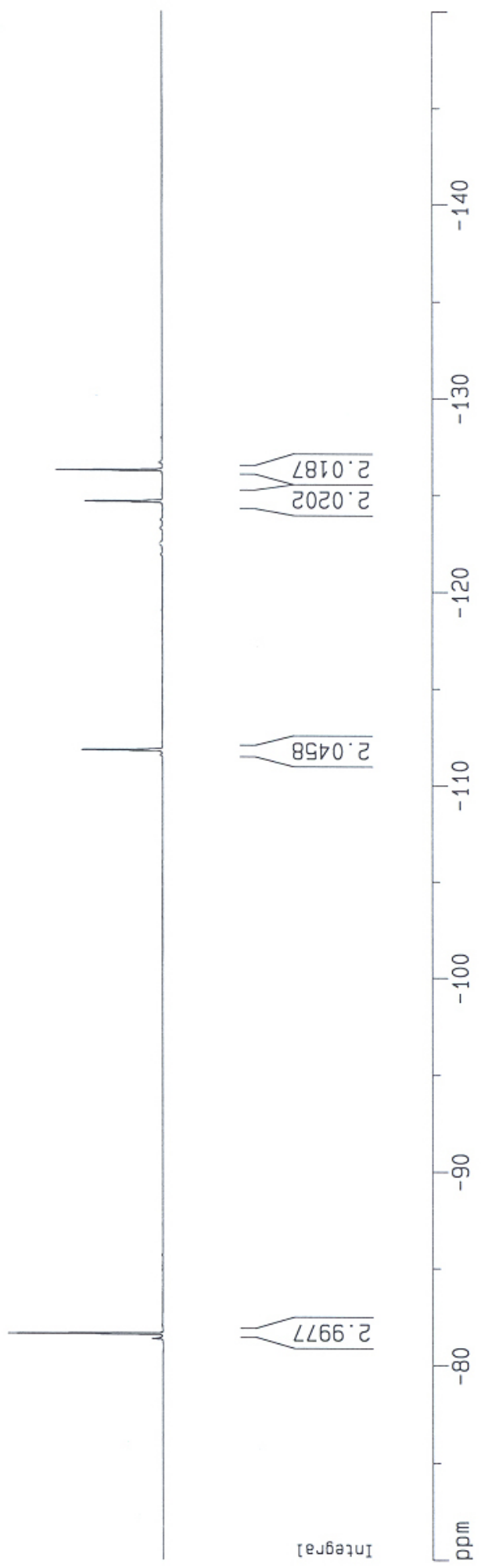

Figure S11: ${ }^{19} \mathrm{~F}$ NMR of $\mathbf{5 a}$ 

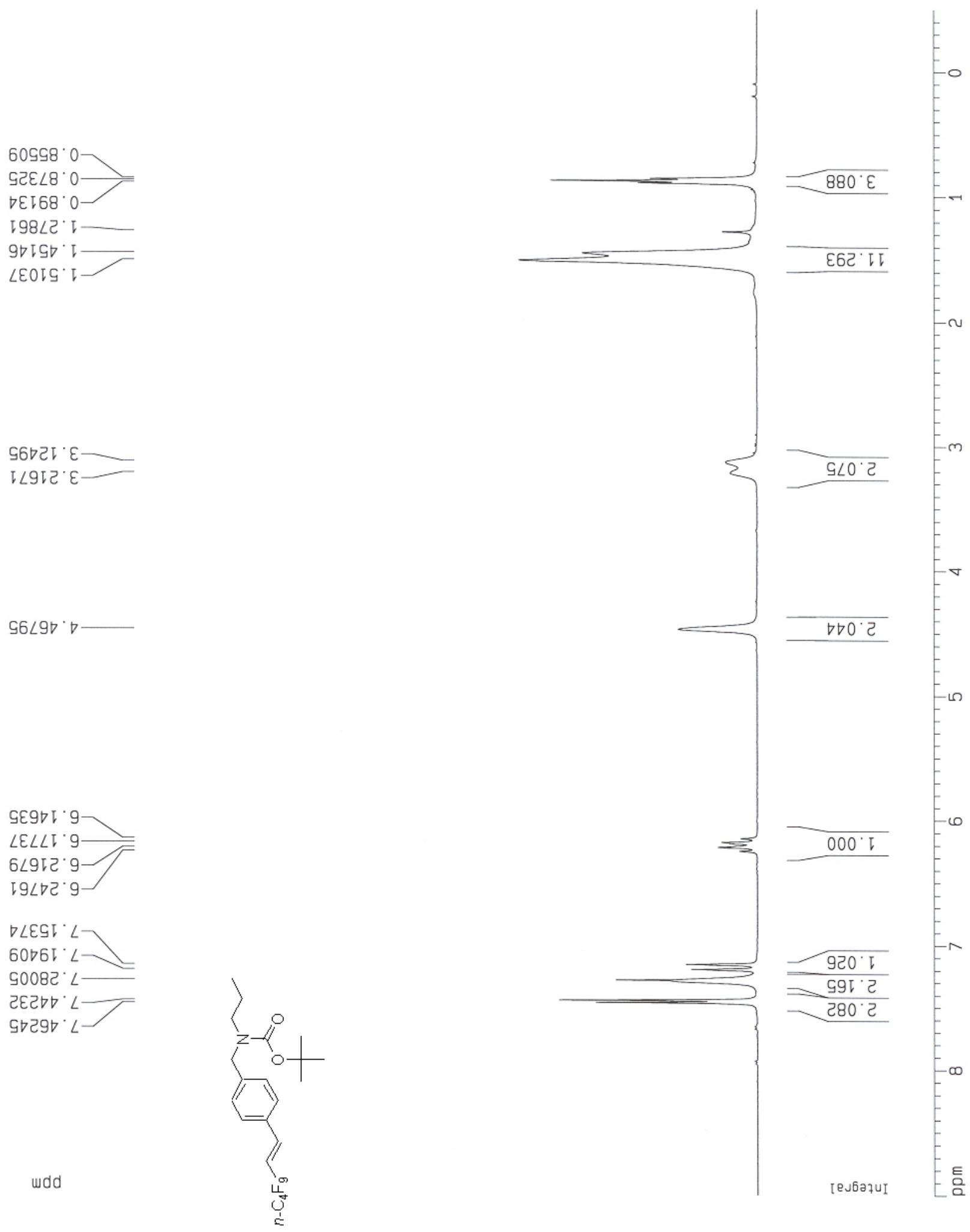

Figure S12: ${ }^{1} \mathrm{H}$ NMR of $\mathbf{5 a}$ 

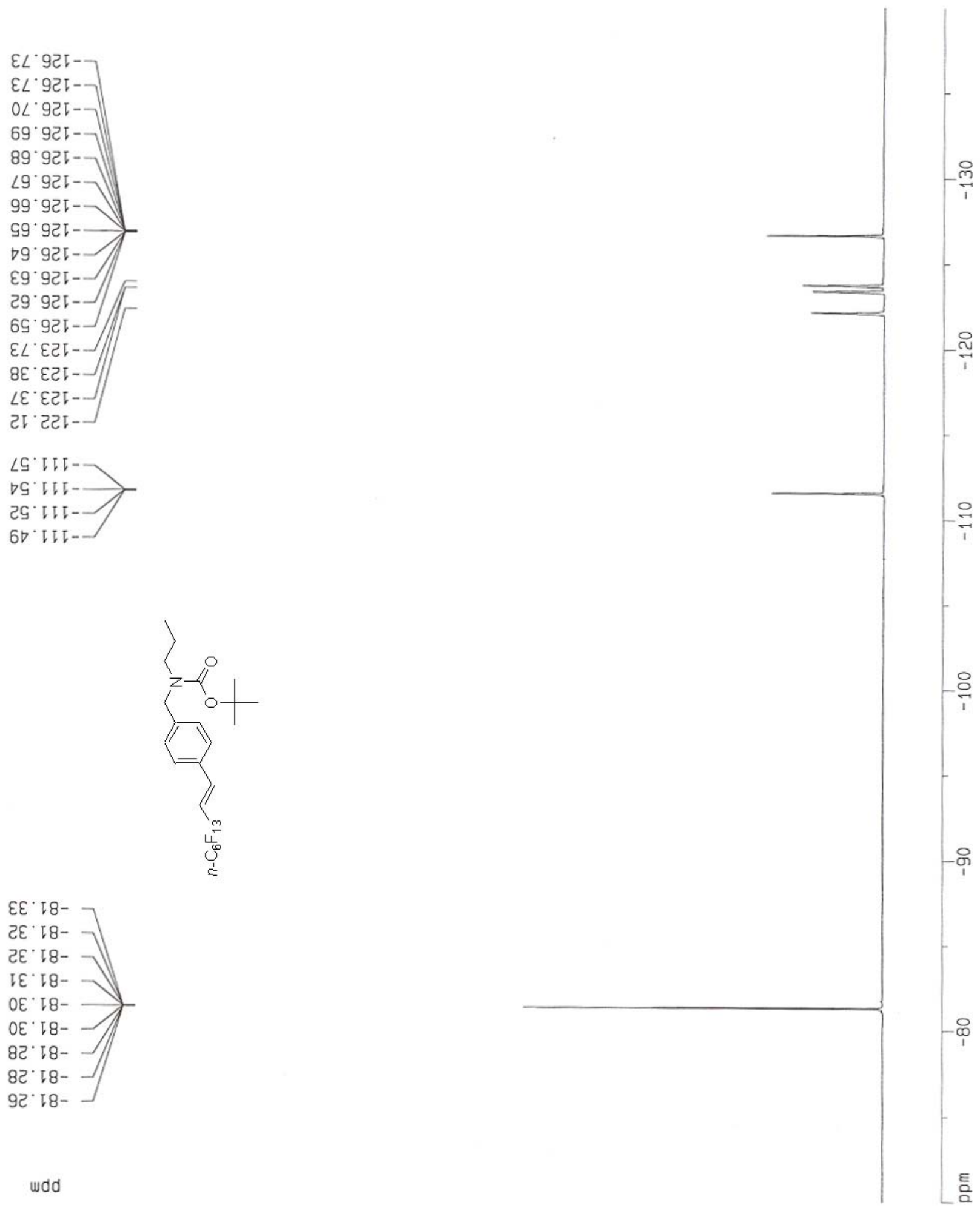

Figure S13: ${ }^{19} \mathrm{~F}$ NMR of $\mathbf{5 b}$ 


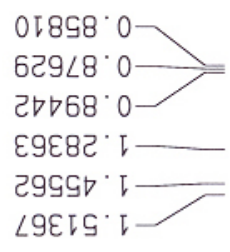

EG6टा ' $\varepsilon$

g9grट' $\varepsilon$

SEE $\angle \nabla^{\circ} \nabla$

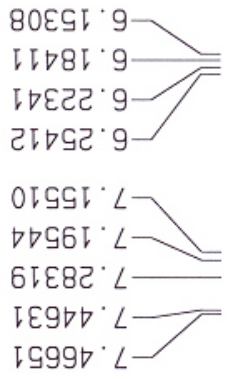

wdd

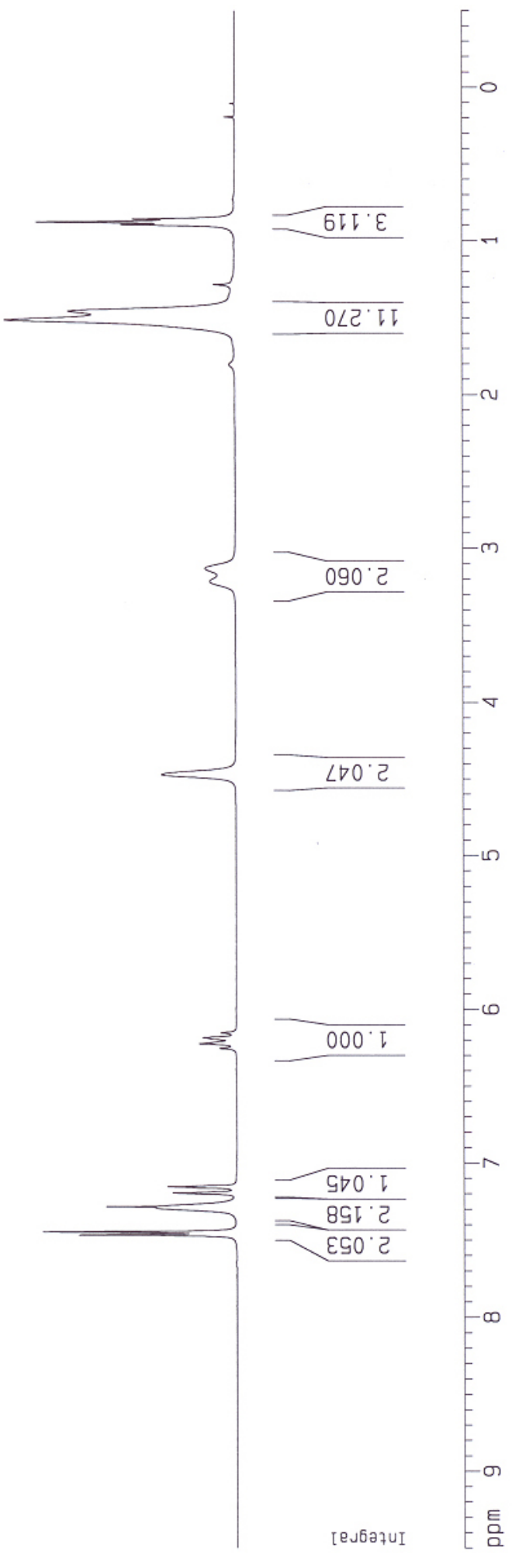

Figure S14: ${ }^{1} \mathrm{H}$ NMR of $\mathbf{5 b}$ $\stackrel{1}{\infty}_{i}^{\infty}$

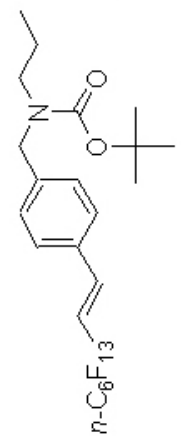



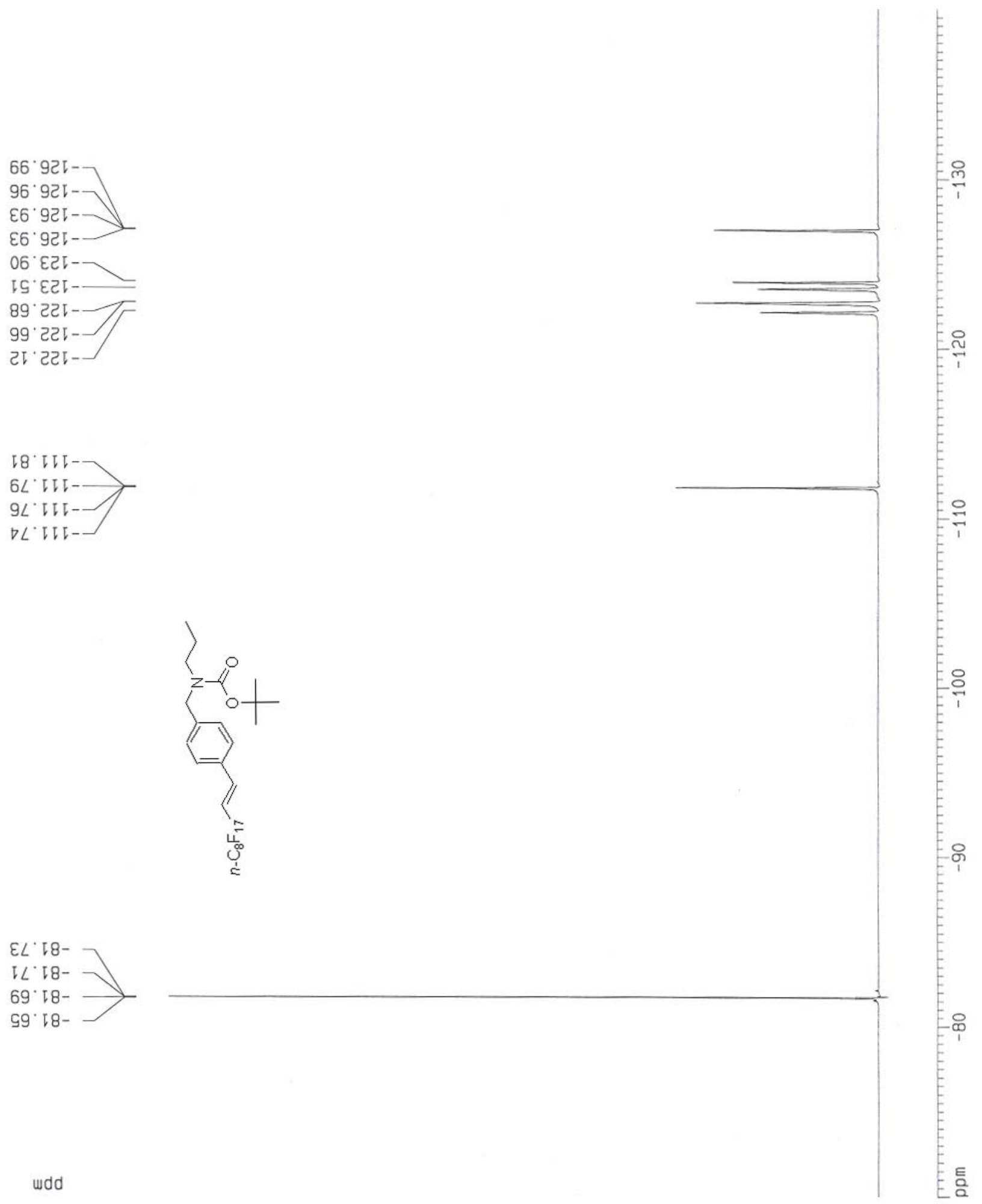

Figure S15: ${ }^{19} \mathrm{~F}$ NMR of $\mathbf{5 c}$ 

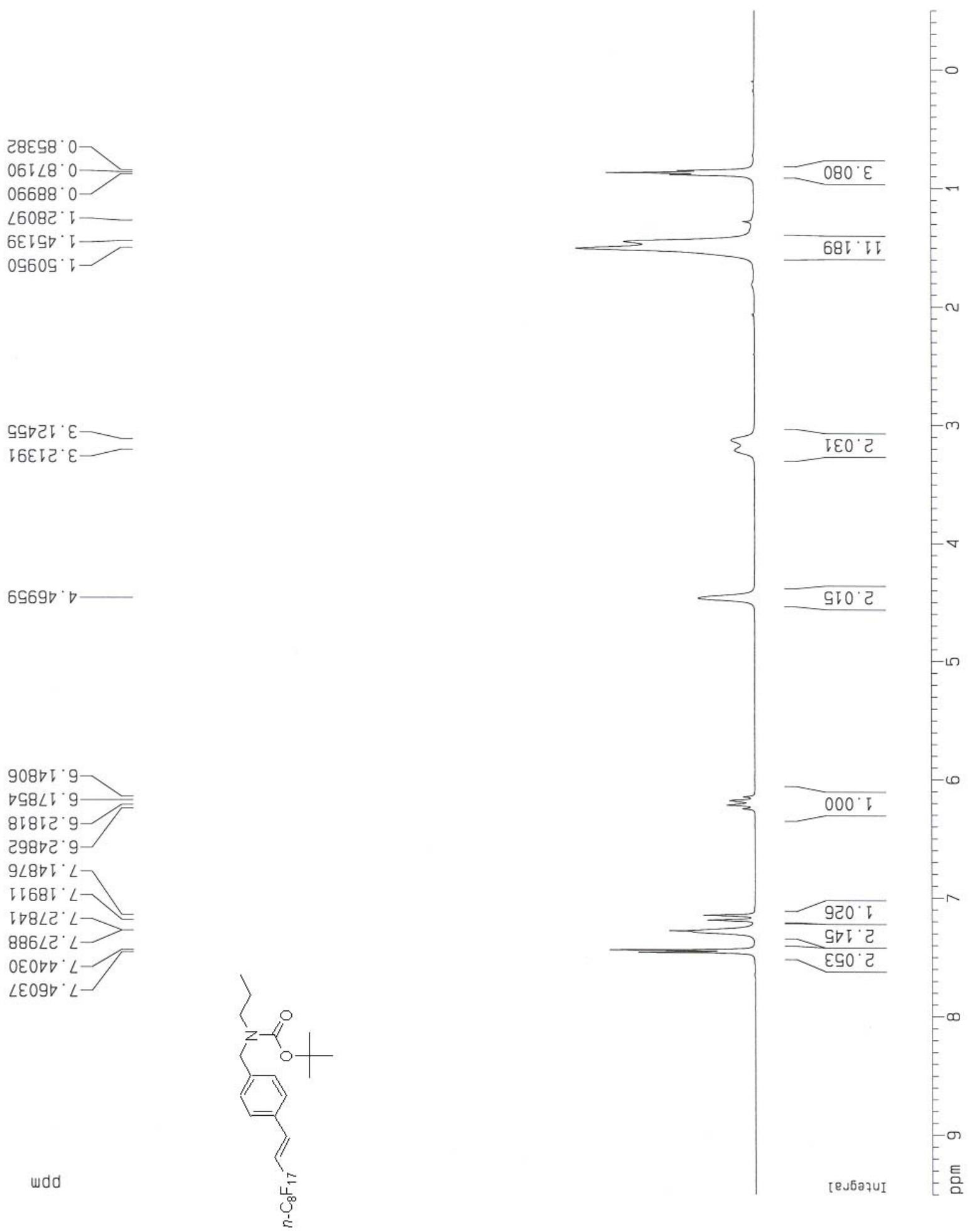

Figure S16: ${ }^{1} \mathrm{H}$ NMR of $\mathbf{5 c}$ 
ह9. 9ट I-

ट9.9टा-

$89^{\circ} \varepsilon 己\lceil-$

ટટ·દटा-

$8 \varepsilon \cdot ट ट ा-$

9 ‘ ટટા-

$88 \cdot 151-$

$\angle G^{\circ} \tau \tau-$ 呵调 IG'䜣-

Oย โ8-

$8 \mathrm{C}^{\prime} 18-$ $9 \mathcal{2}^{\prime}$ เ8-

wdd

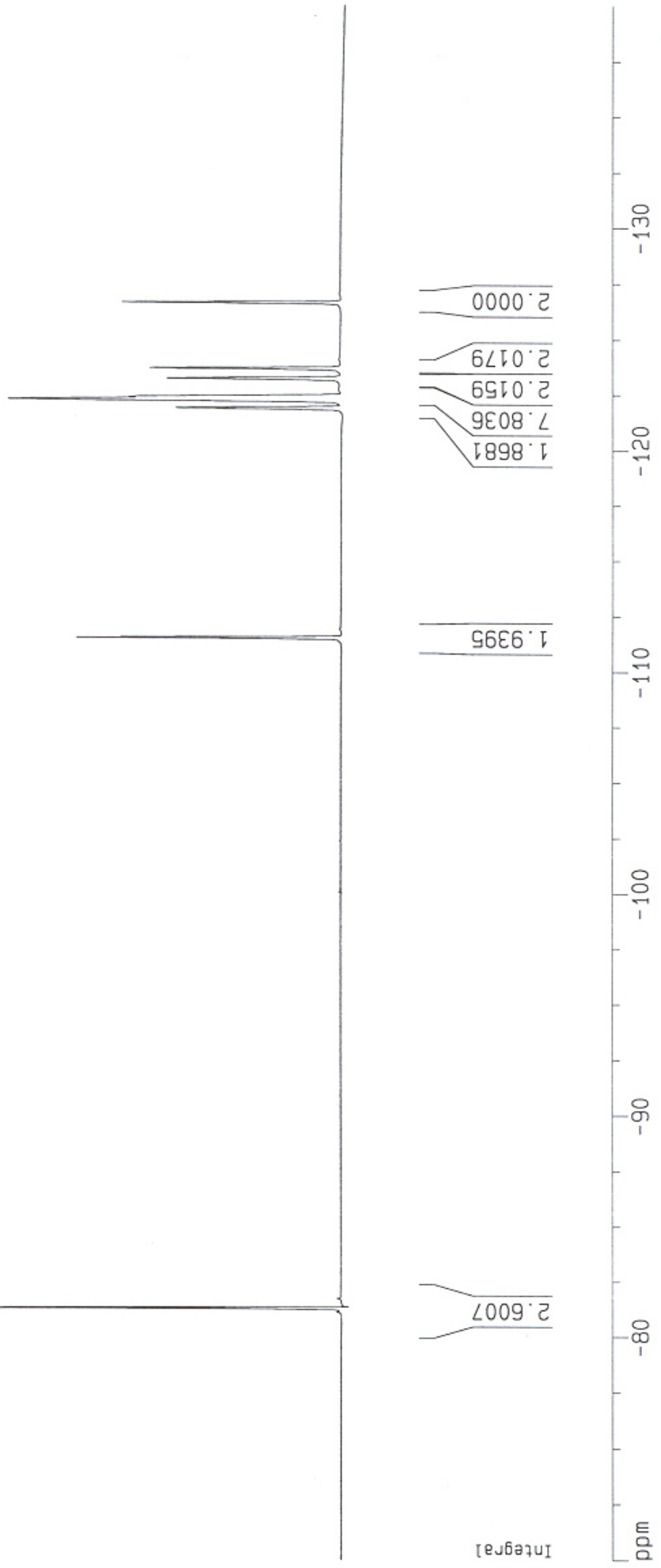

Figure S17: ${ }^{19} \mathrm{~F}$ NMR of $\mathbf{5 d}$ 


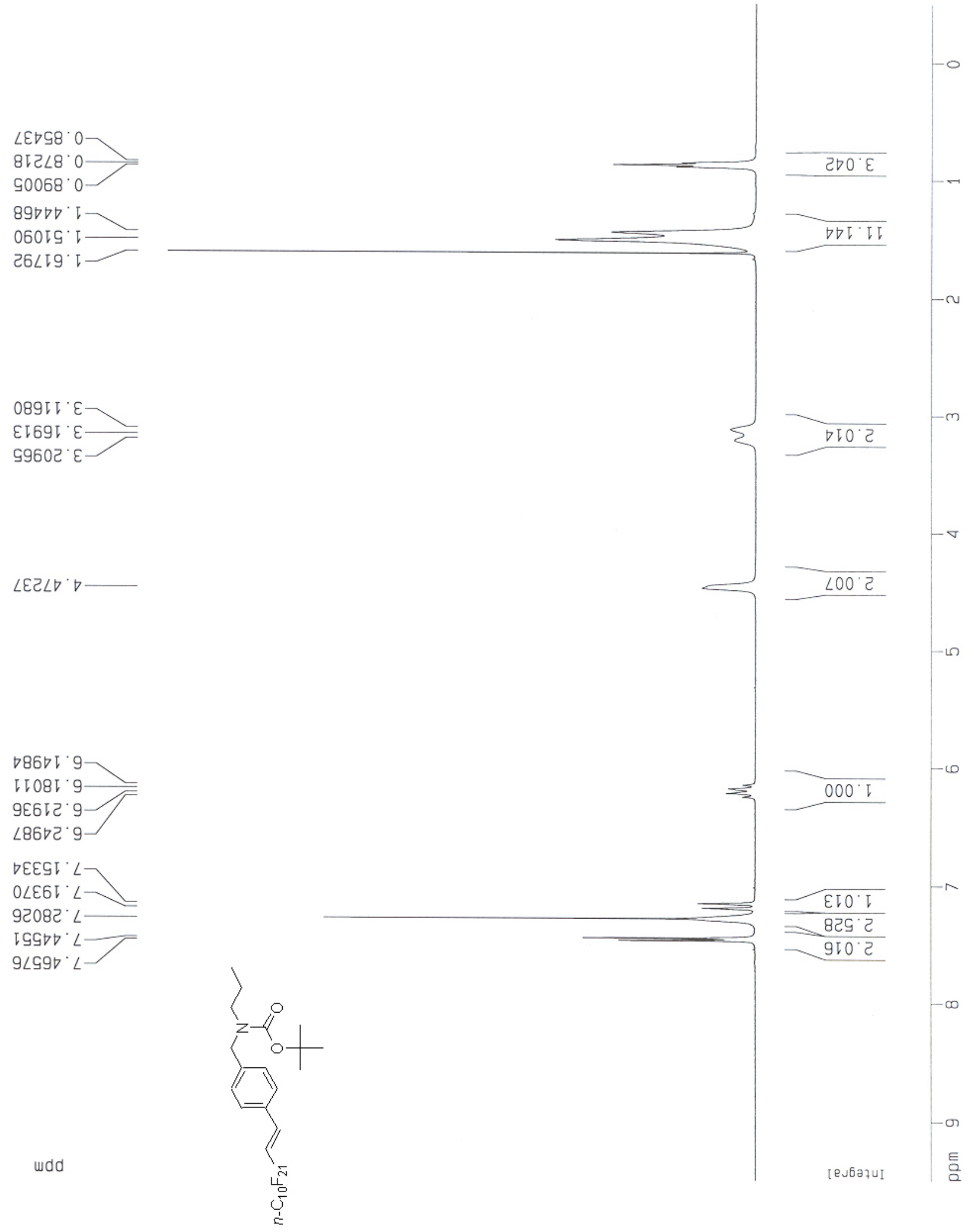

Figure S18: ${ }^{1} \mathrm{H}$ NMR of $\mathbf{5 d}$ 

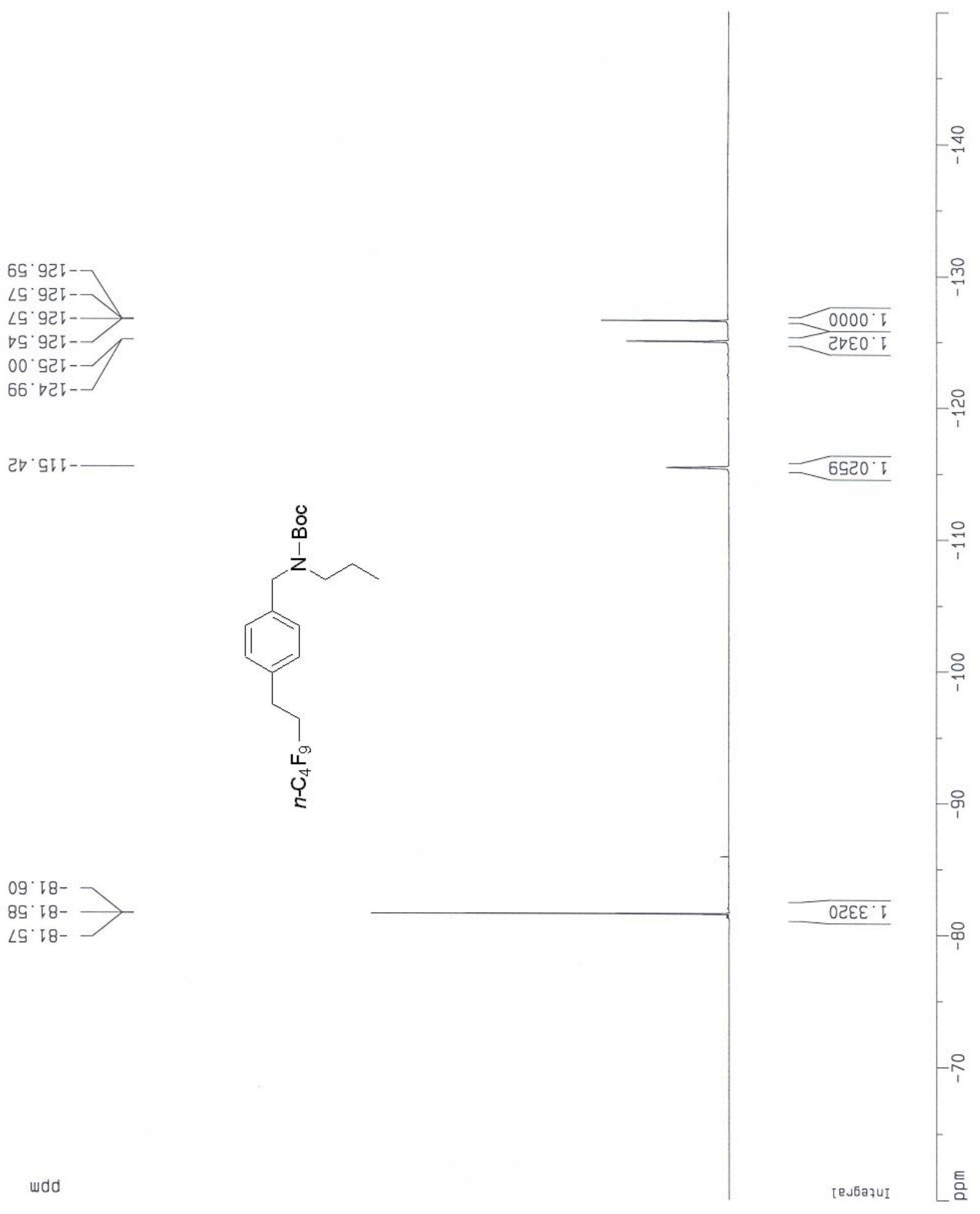

wdd

Figure S19: ${ }^{19} \mathrm{~F}$ NMR of $6 a$ 


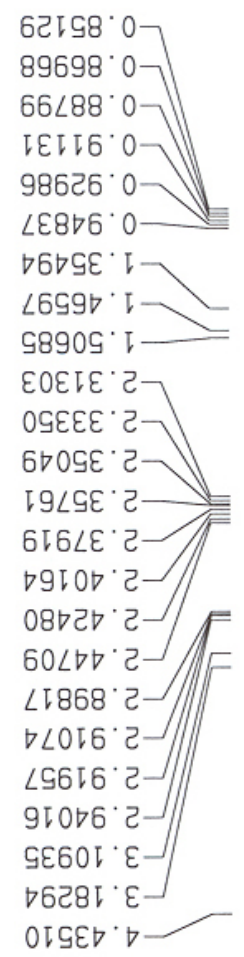

$\angle \forall \nabla \nabla I \cdot L$
$8669 I^{\circ} \angle$
I $688 I^{\circ} \cdot L$

wdd

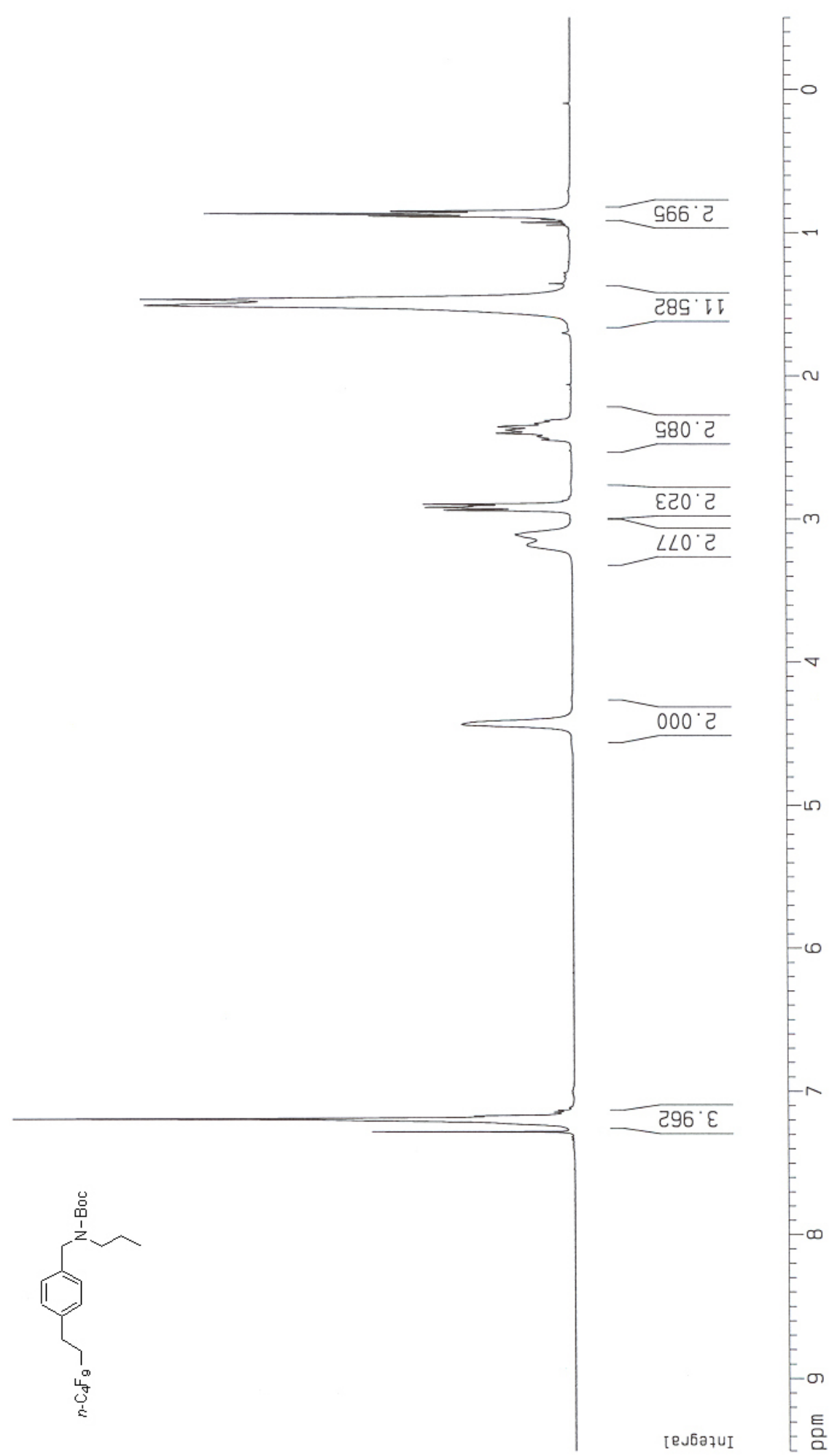

Figure S20: ${ }^{1} \mathrm{H}$ NMR of $\mathbf{6 a}$ 

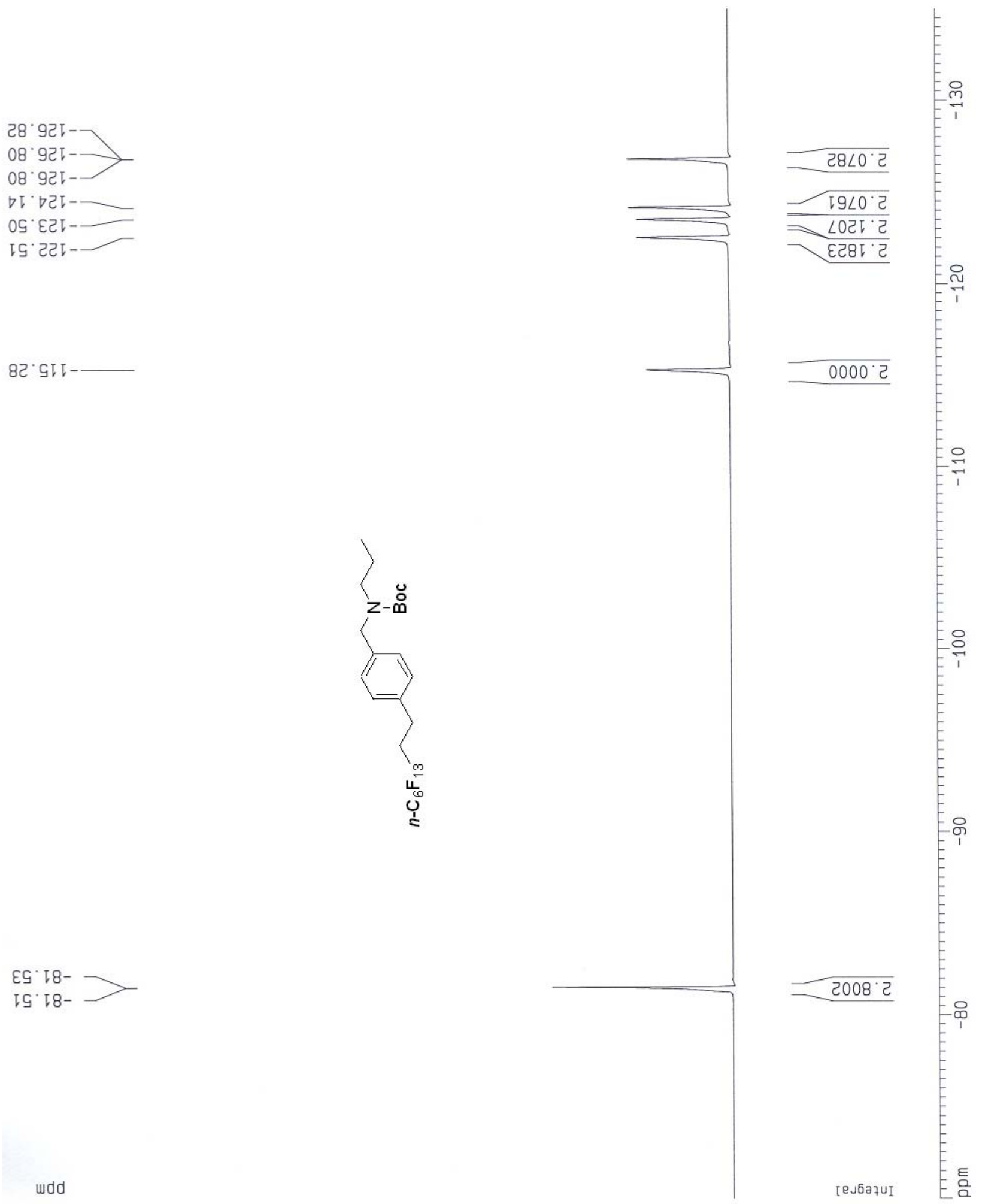

EG เ8-

Figure S21: ${ }^{19}$ F NMR of $\mathbf{6 b}$ 


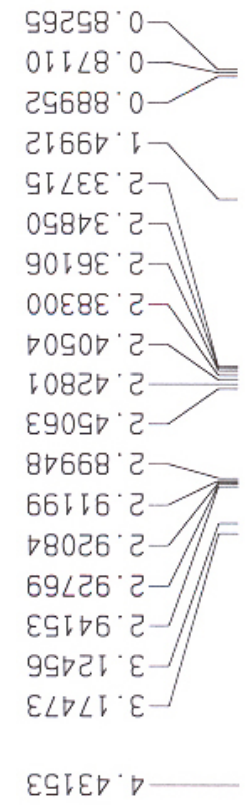

EGLEจ

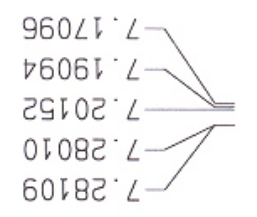

wdd

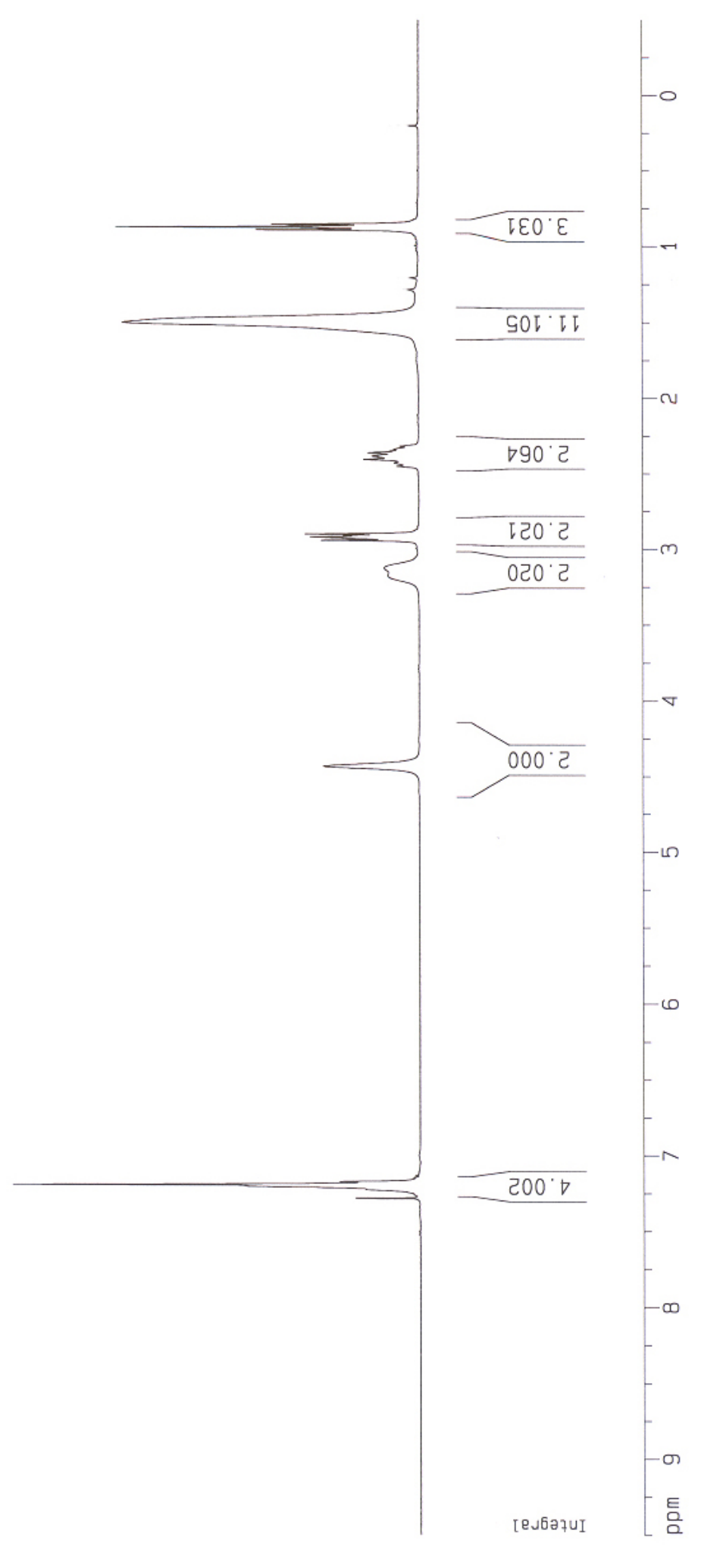

Figure S22: ${ }^{1} \mathrm{H}$ NMR of $\mathbf{6 b}$ 


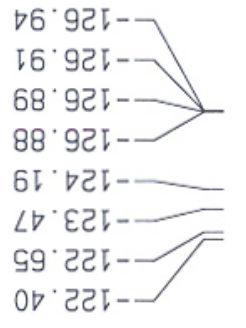

8E'GL-

$\angle 9^{\circ}$. 8 99. โ8ह9' เ8-

udd

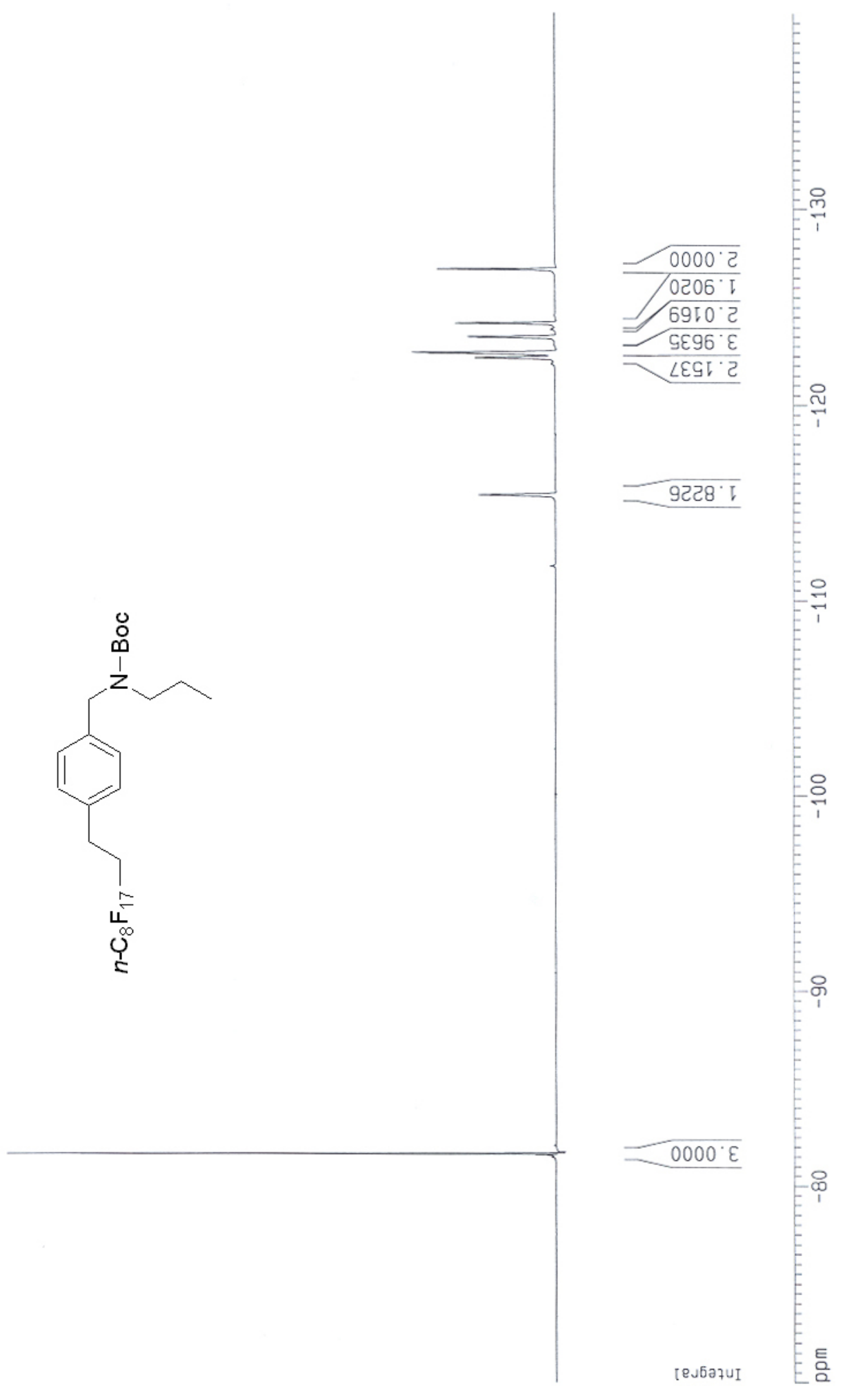

Figure S23: ${ }^{19} \mathrm{~F}$ NMR of $\mathbf{6 c}$ 
ОЕЕ૬ $8^{\circ}$

टGL $\angle 8^{\circ} 0$

$89688^{\circ} 0$

9ع99 ${ }^{\circ}$.

จGIG'

$8 \nabla \angle \varepsilon \varepsilon^{\prime} \circlearrowright-$

$8 \nabla \nabla G \varepsilon^{\prime} \mathrm{C}$

Iट9LE' ट

$\angle 986 \varepsilon^{\circ} \mathrm{C}$

टहाट॰ ट

हटદ68. ट-

II906.

$\nabla 9 \square\left[6^{\circ} \mathrm{C}\right.$

80૬ह6. ट-

$\nabla 0 \angle$ T' $E$

५จ๑๐ ' $\varepsilon$

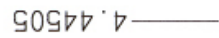

टह89 ' $\angle$

$0 \angle 88 I^{\circ} \angle-$

$\triangle 1902^{*} \angle$

$6 \varepsilon \angle G 己^{*} \angle$

$8 \angle 6 \angle C^{\circ} \angle$

wdd
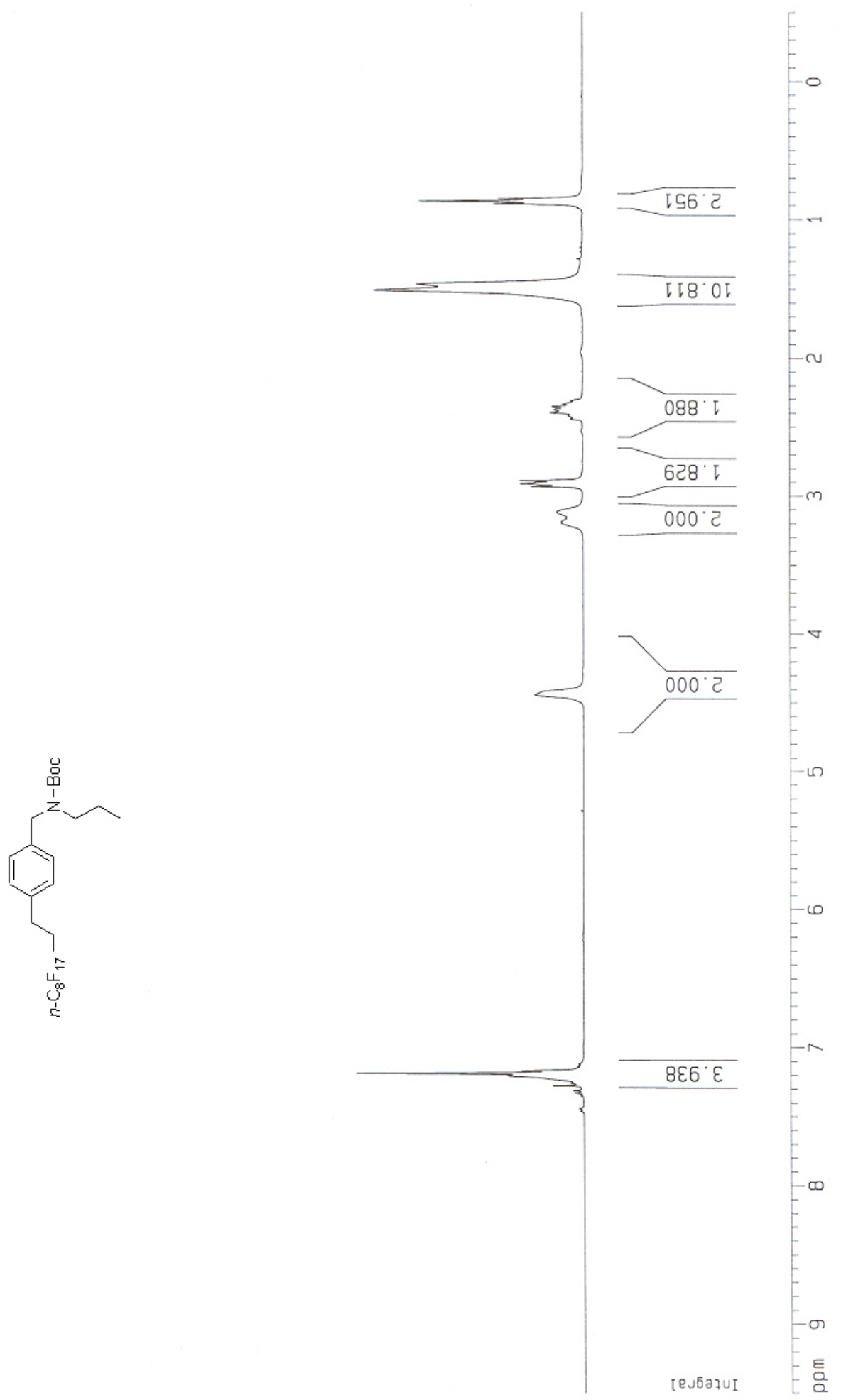

Figure S24: ${ }^{1} \mathrm{H}$ NMR of $\mathbf{6 c}$ 


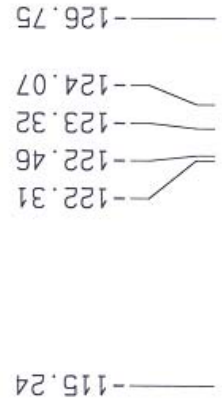

与ั เ8ยจ โ8讨朋-

wdd

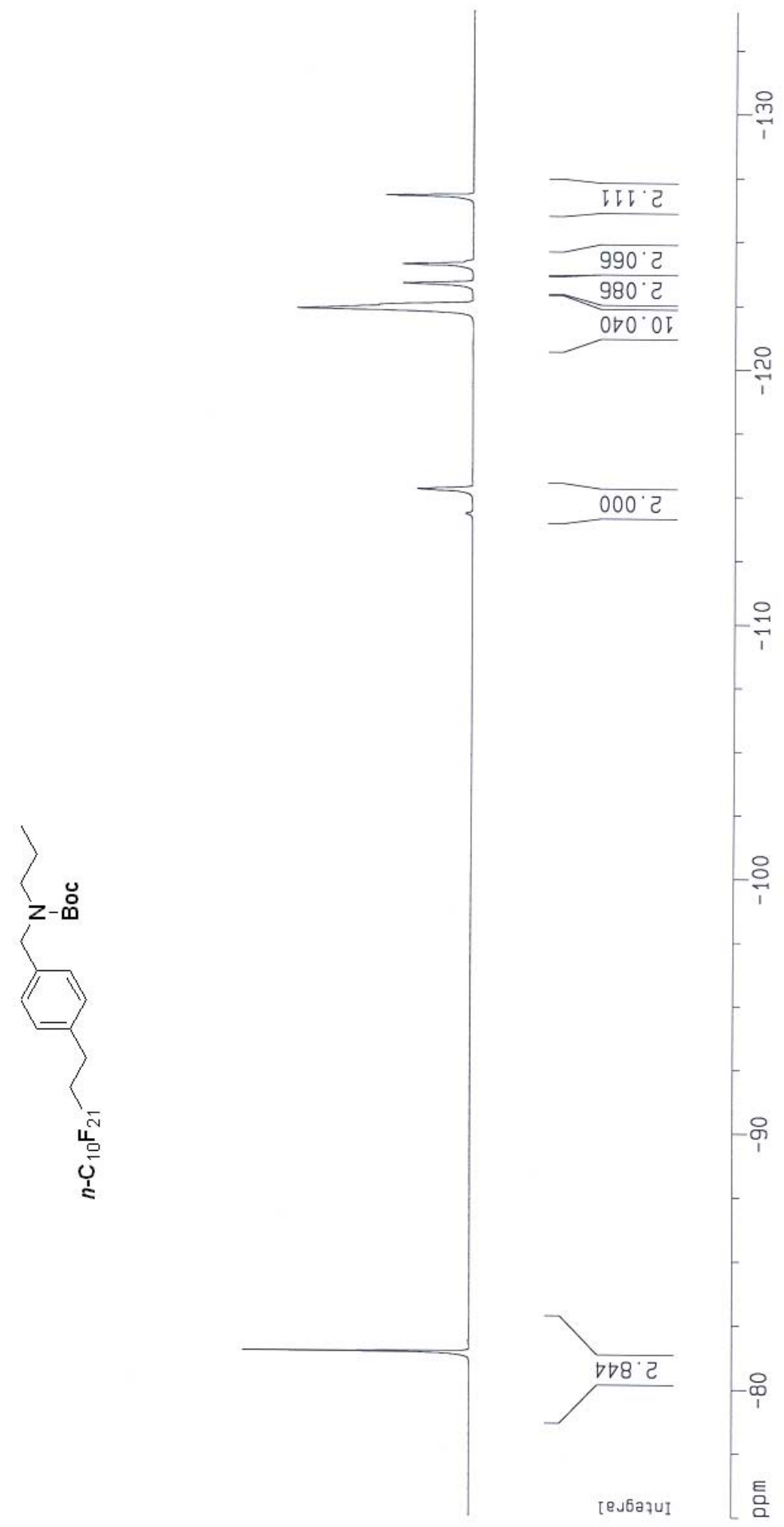

Figure S25: ${ }^{19} \mathrm{~F}$ NMR of $\mathbf{6 d}$ 

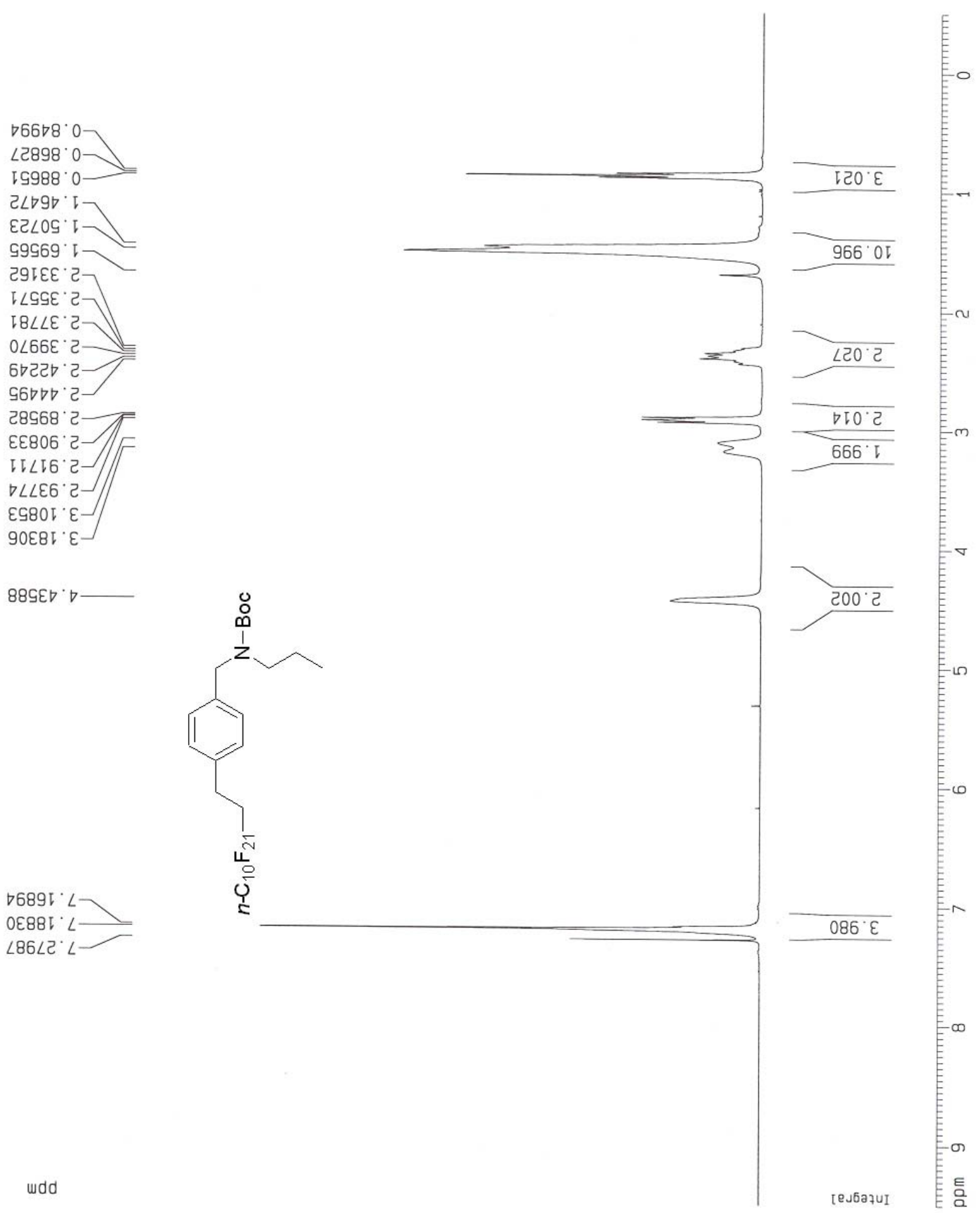

wdd

Figure S26: ${ }^{1} \mathrm{H}$ NMR of $\mathbf{6 d}$ 

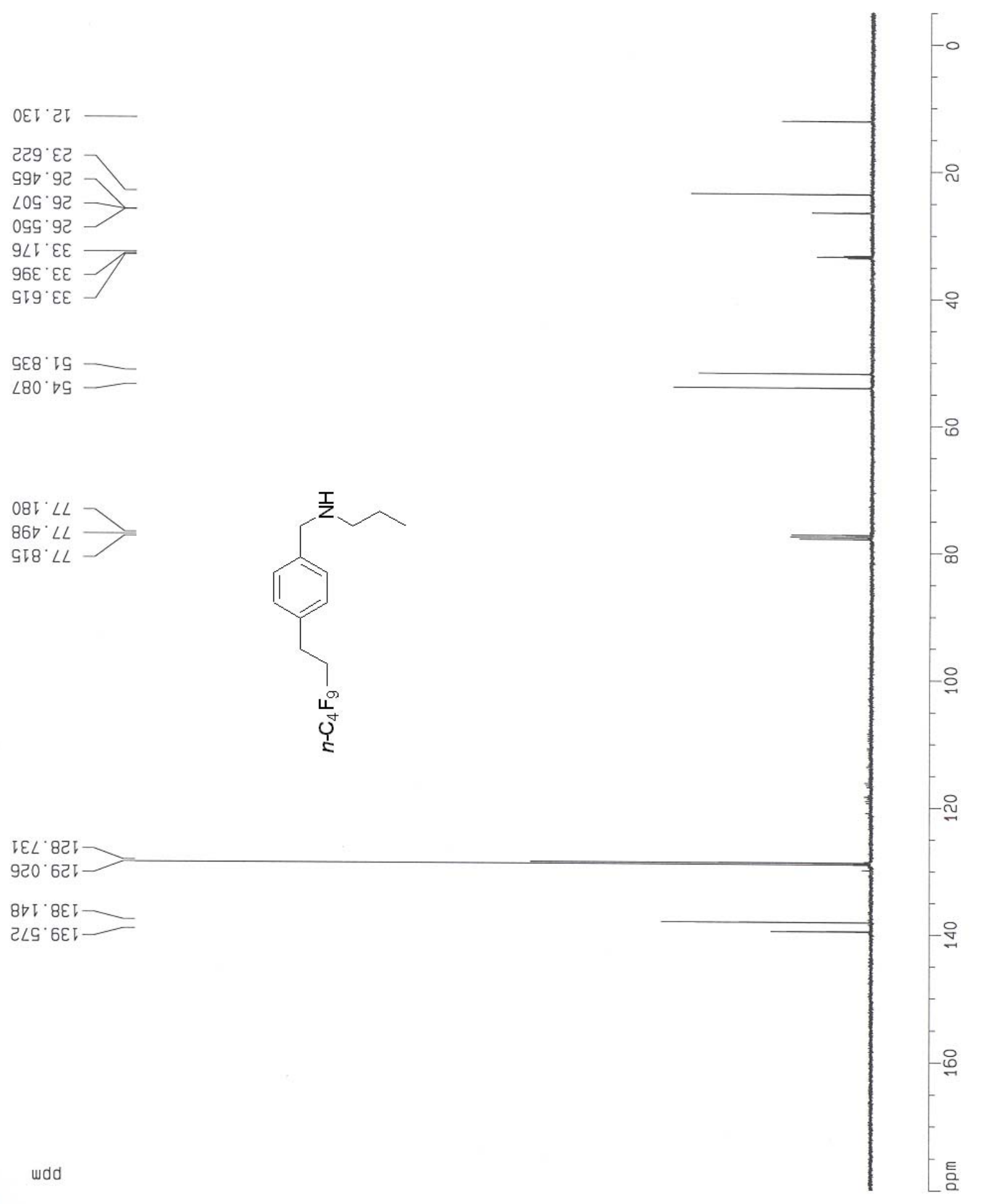

IEL 82 I-

920.625

$8 \nabla \downarrow \cdot 8 \varepsilon \downarrow-$

ZLG.6EI

wdd

Figure S27: ${ }^{13} \mathrm{C}$ NMR of 7a 


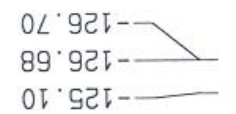

DG. GI $\longrightarrow$
टG. GI $\longrightarrow$

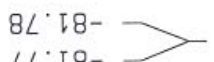

LL' ᄂ8-

wdd

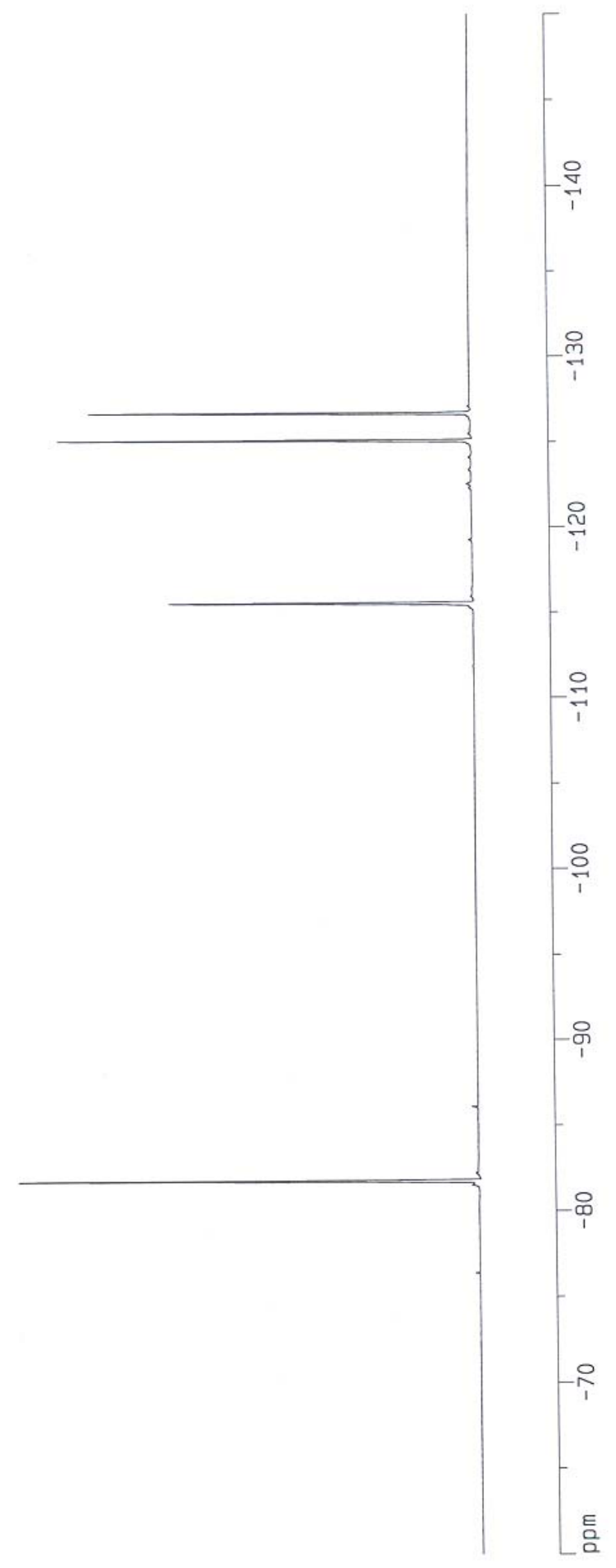

Figure S28: ${ }^{19}$ F NMR of $7 \mathbf{a}$ 


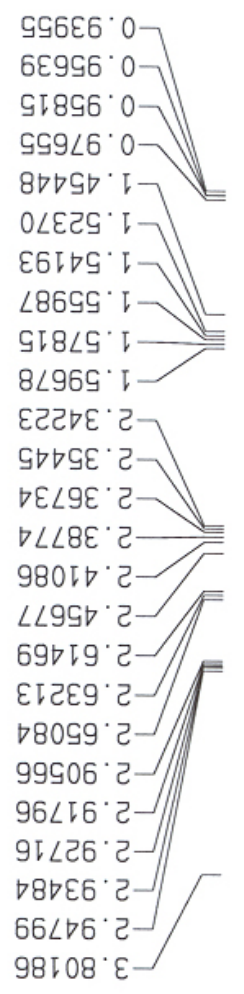

$01881^{\circ} L$ G1802. $\angle$ $\angle 1082 \cdot \angle$ $8980 \varepsilon^{\prime} \angle$ О૪৪टह $\angle-$

wdd

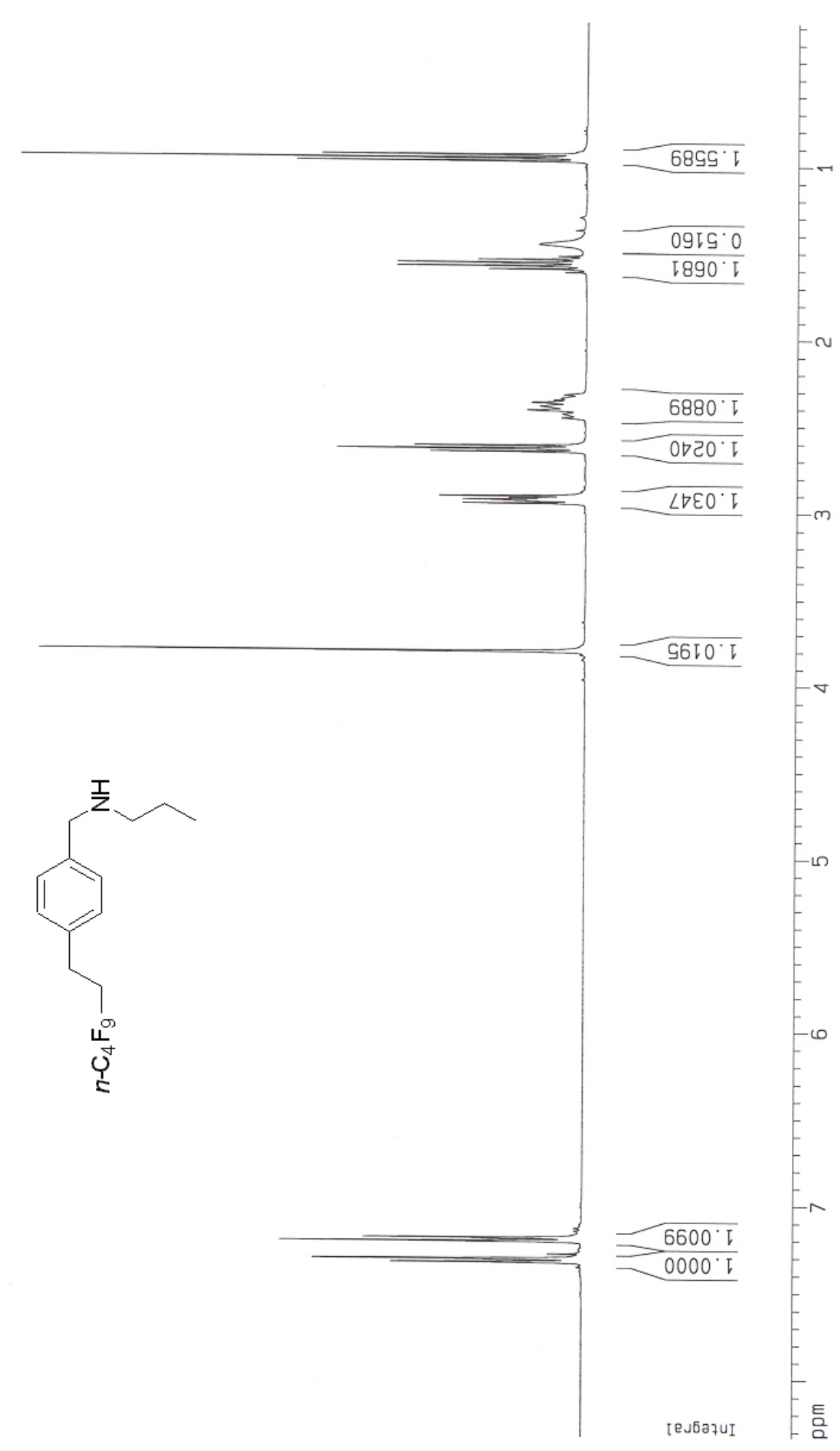

Figure S29: ${ }^{1} \mathrm{H}$ NMR of 7a 


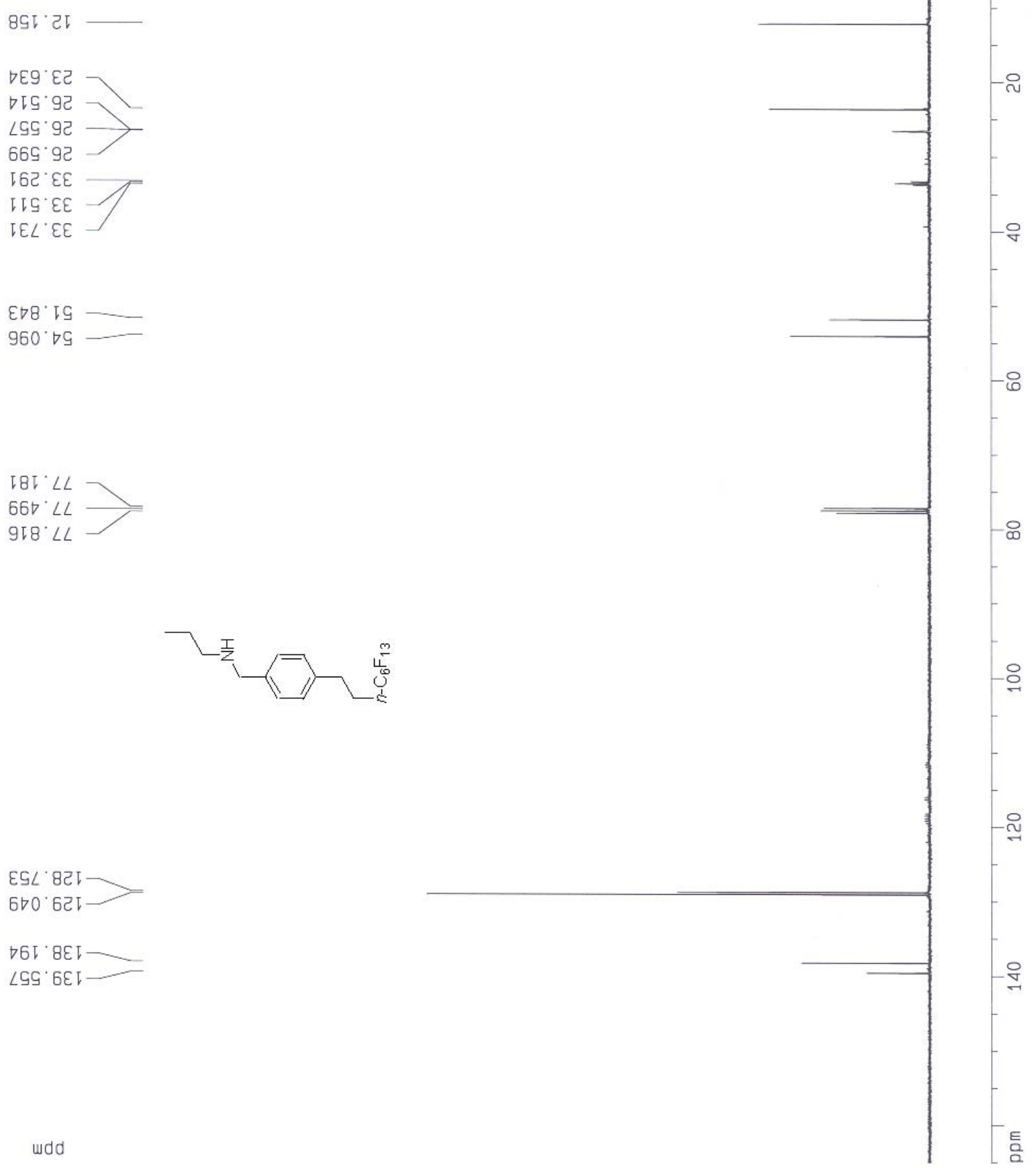

Figure S30: ${ }^{13} \mathrm{C}$ NMR of $7 \mathbf{b}$ 

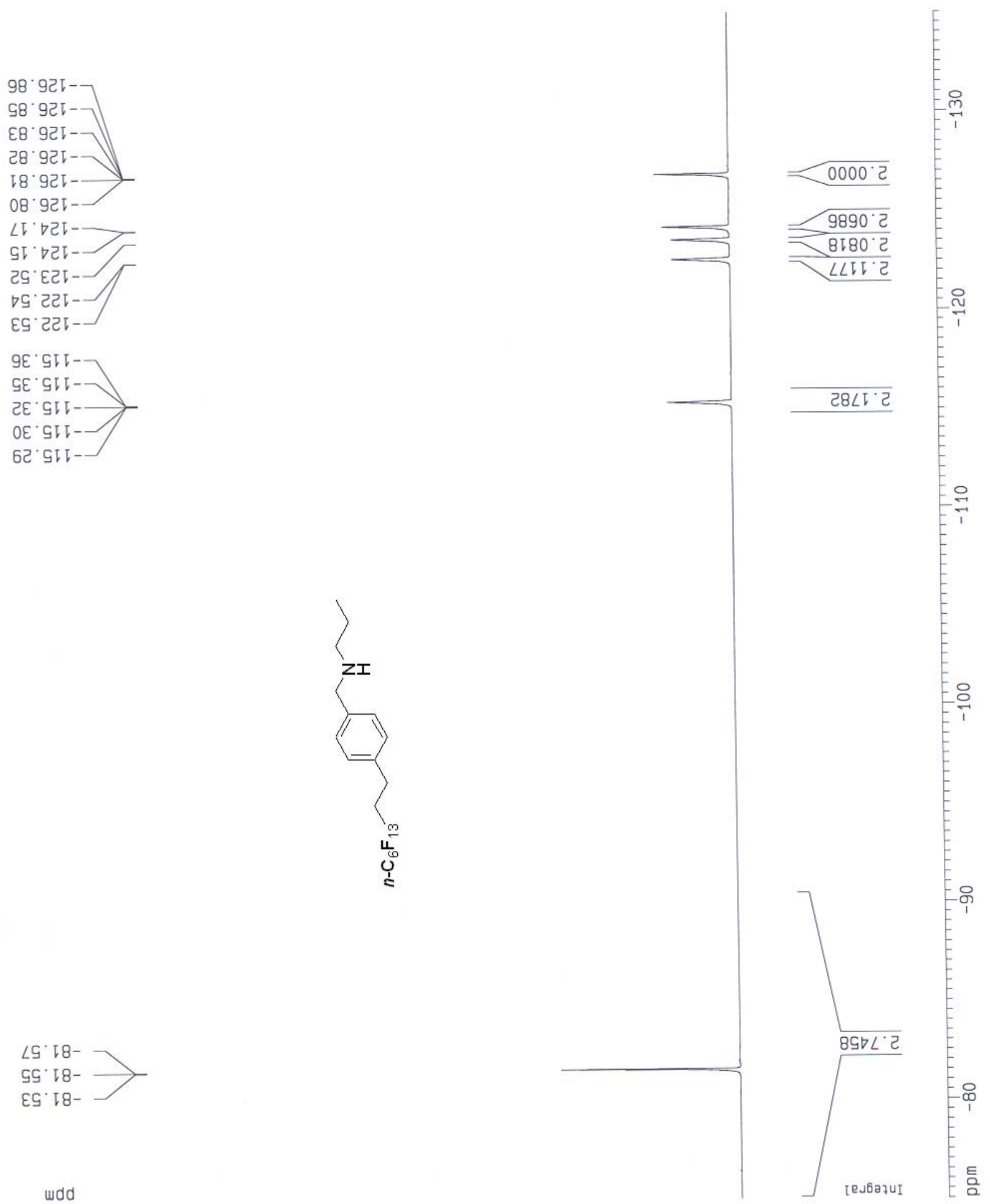

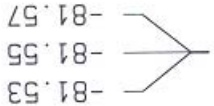

wdd

Figure S31: ${ }^{19} \mathrm{~F}$ NMR of $\mathbf{7 b}$ 


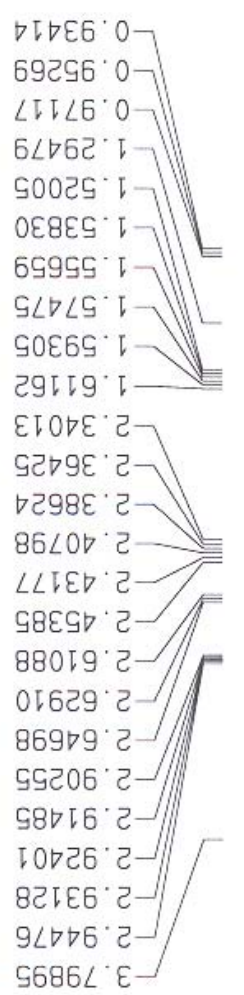

$5686 \angle L^{\circ} \varepsilon-$

$90981^{\circ} L$ ट050 ' $L$ $9 \angle 6 \angle 2 \cdot$

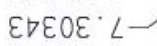
ВЕЕटЕ $\llcorner-$

wdd

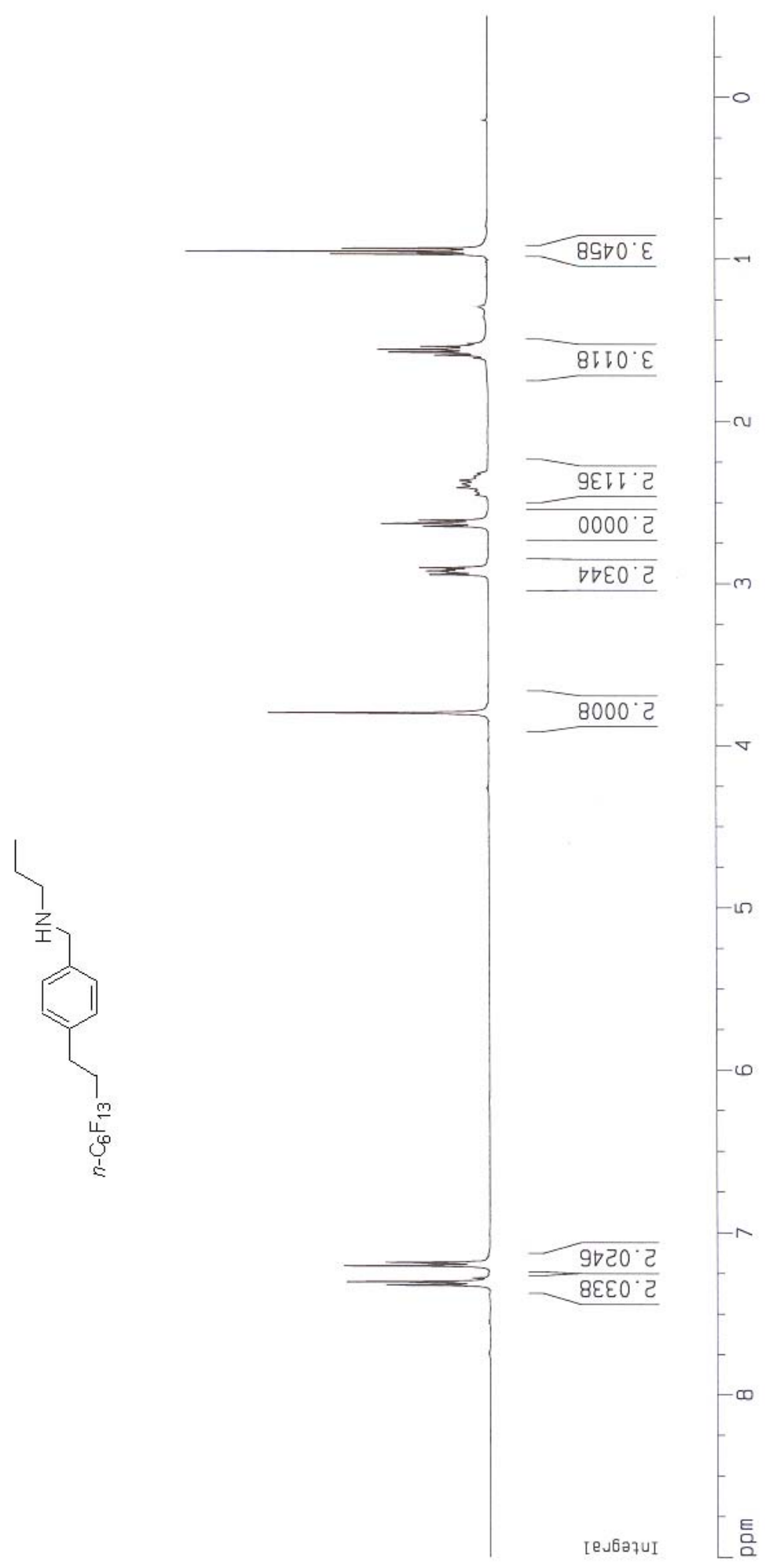

Figure S32: ${ }^{1} \mathrm{H}$ NMR of $\mathbf{7 b}$ 


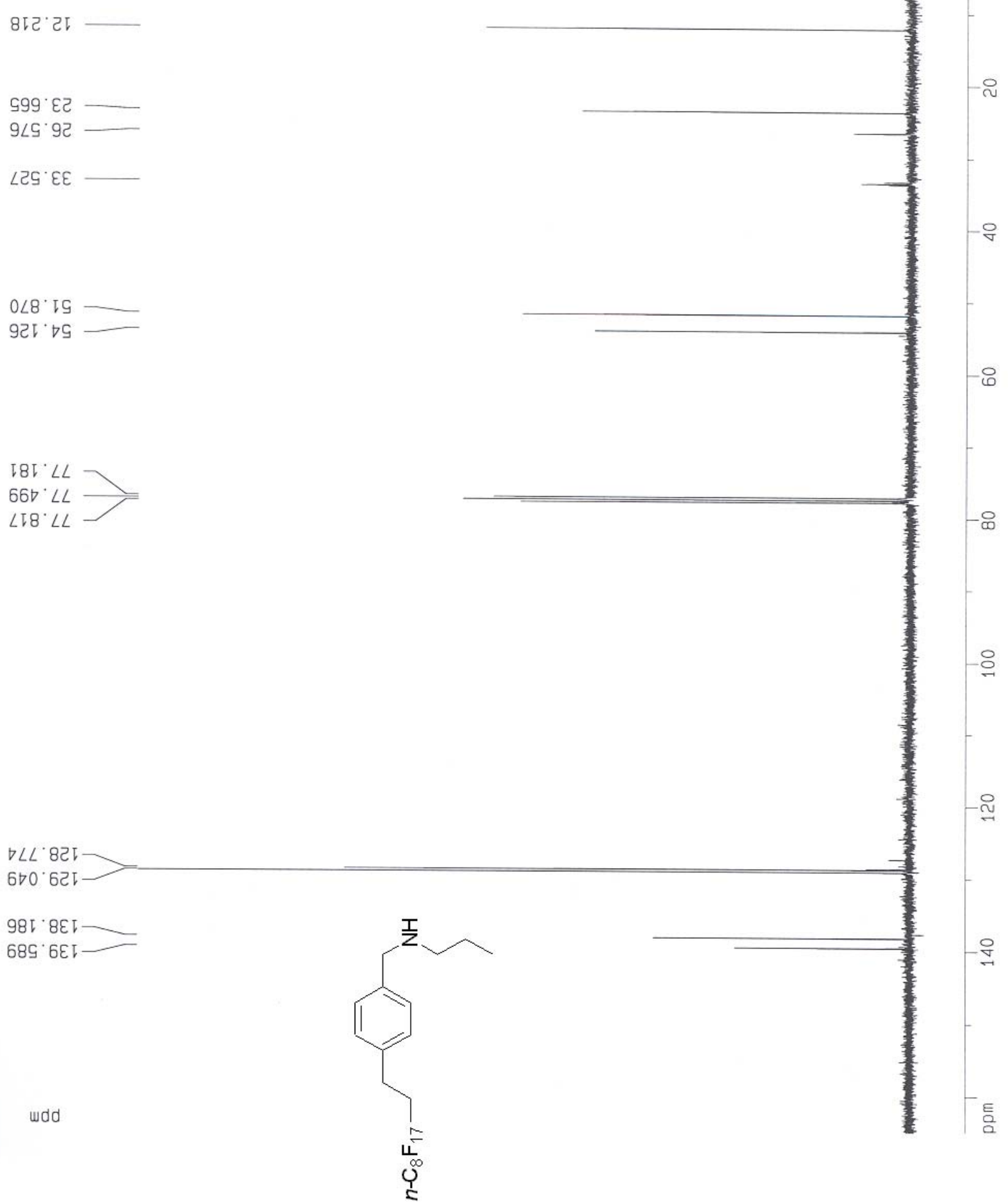

Figure S33: ${ }^{13} \mathrm{C}$ NMR of $7 \mathbf{c}$ 

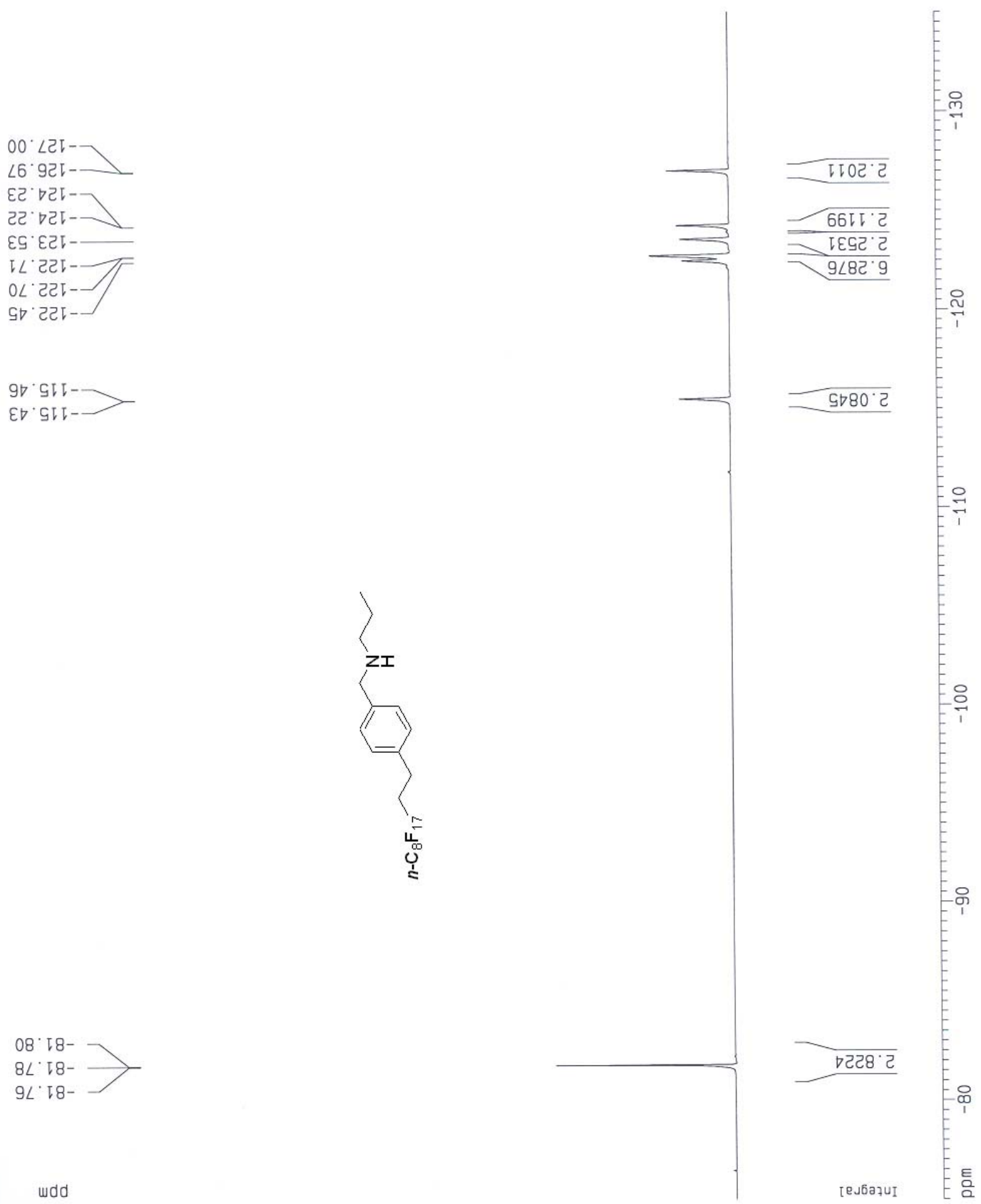

wdd

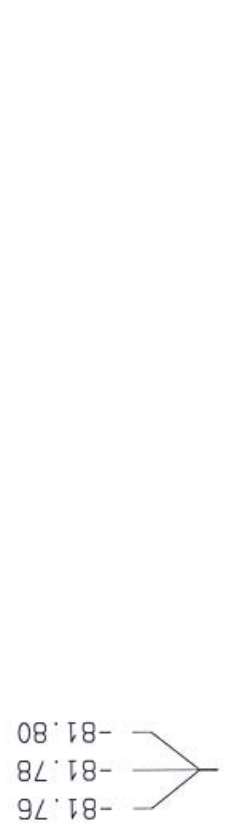

Figure S34: ${ }^{19} \mathrm{~F}$ NMR of $7 \mathbf{c}$ 

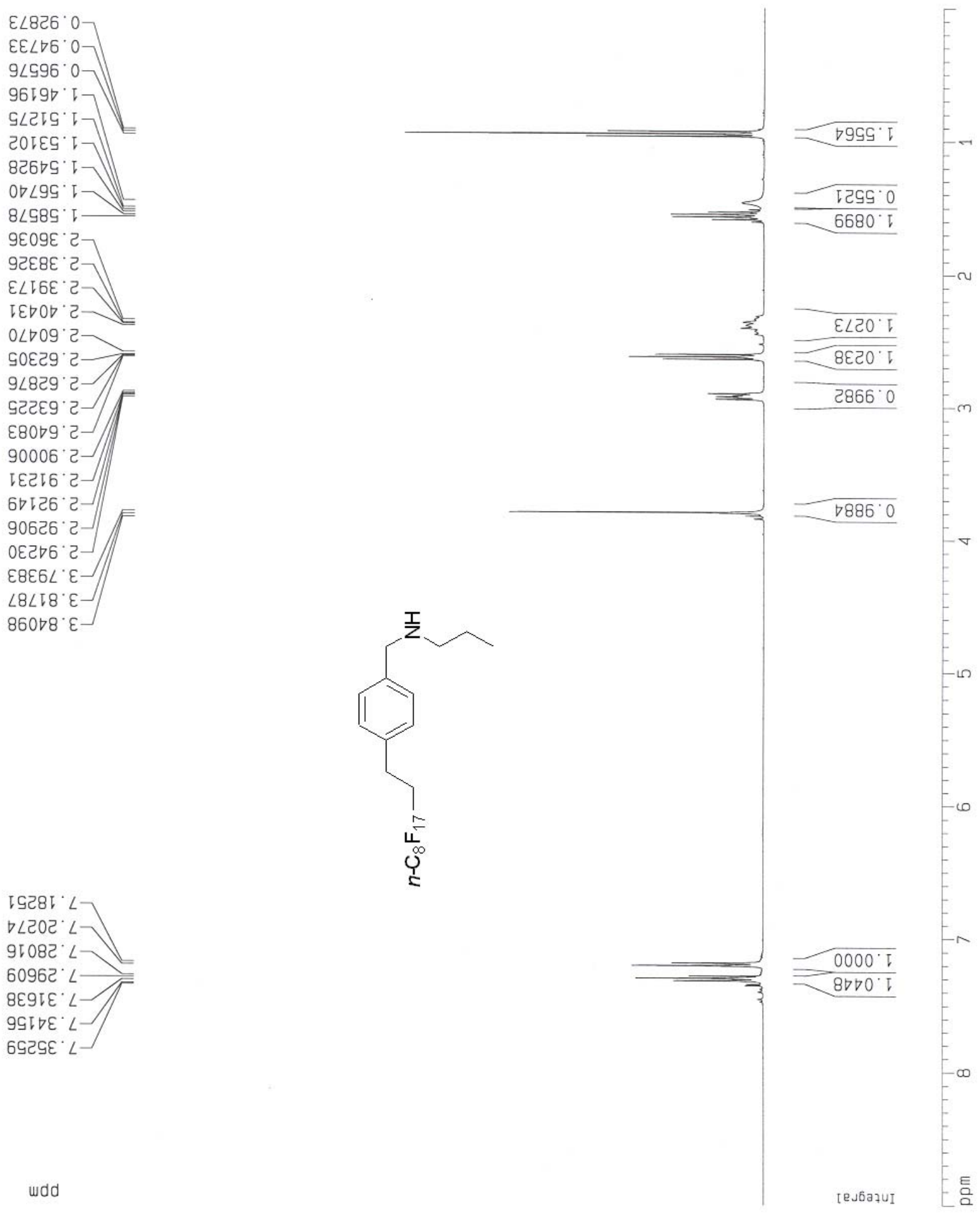

IGE8 . $L$ $\nabla \angle 20 C^{\circ} \angle$ $91082^{\circ} \mathrm{L}$ $60962 \cdot \angle$ В६9เE' $L$ 9GIVE $\angle-$ 6૬ટ૬દ $\llcorner-$

Figure S35: ${ }^{1} \mathrm{H}$ NMR of $\mathbf{7 c}$ 


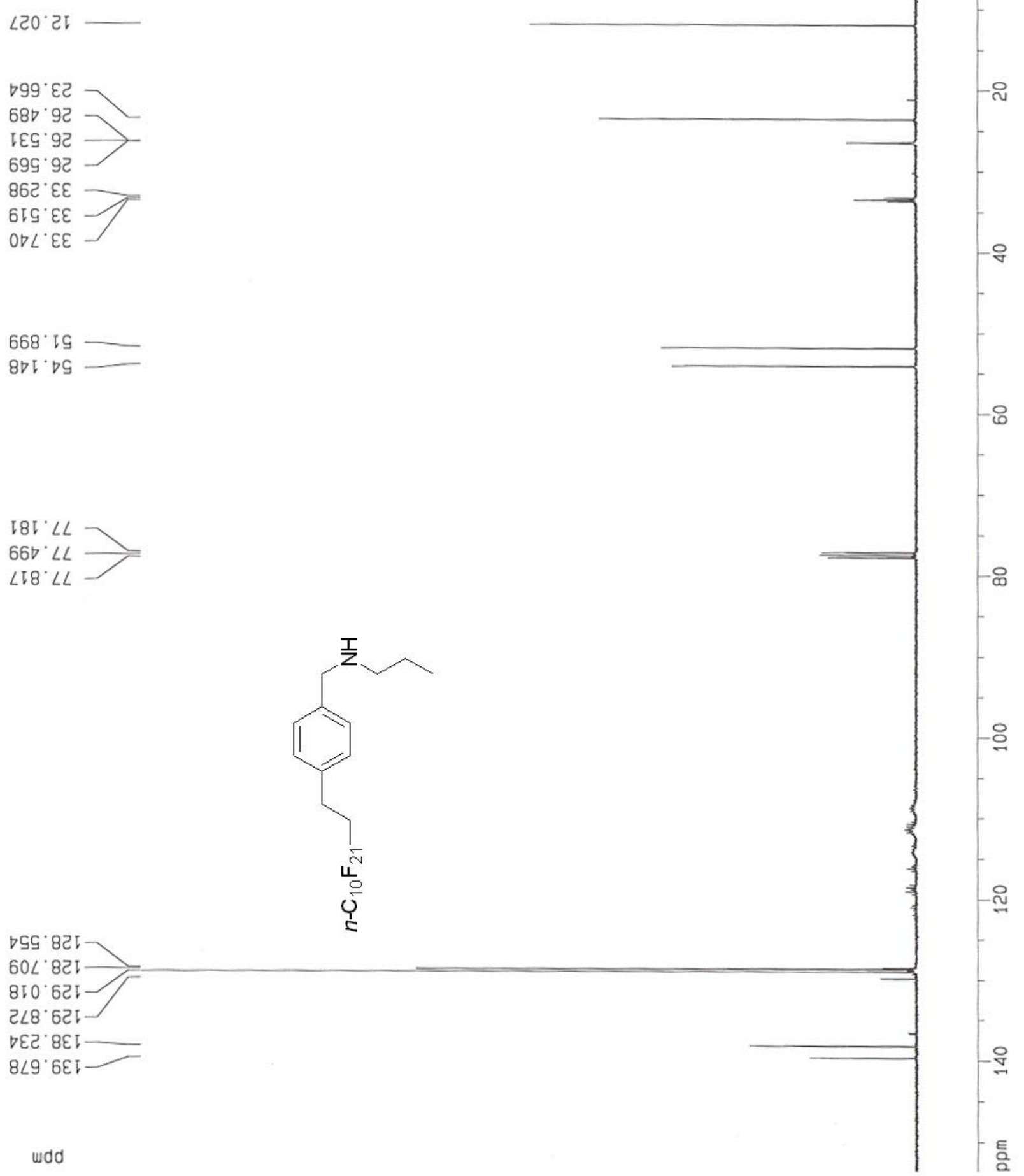

Figure S36: ${ }^{13} \mathrm{C}$ NMR of 7d 


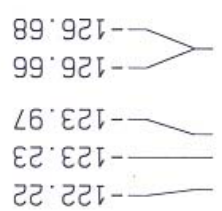

Lट.GLI$8 \tau \cdot 9 t \tau-\longrightarrow$

0ั โ8$8 \varepsilon^{\prime}$. $18-$ $9 \varepsilon^{\prime}$ เ8-

wdd

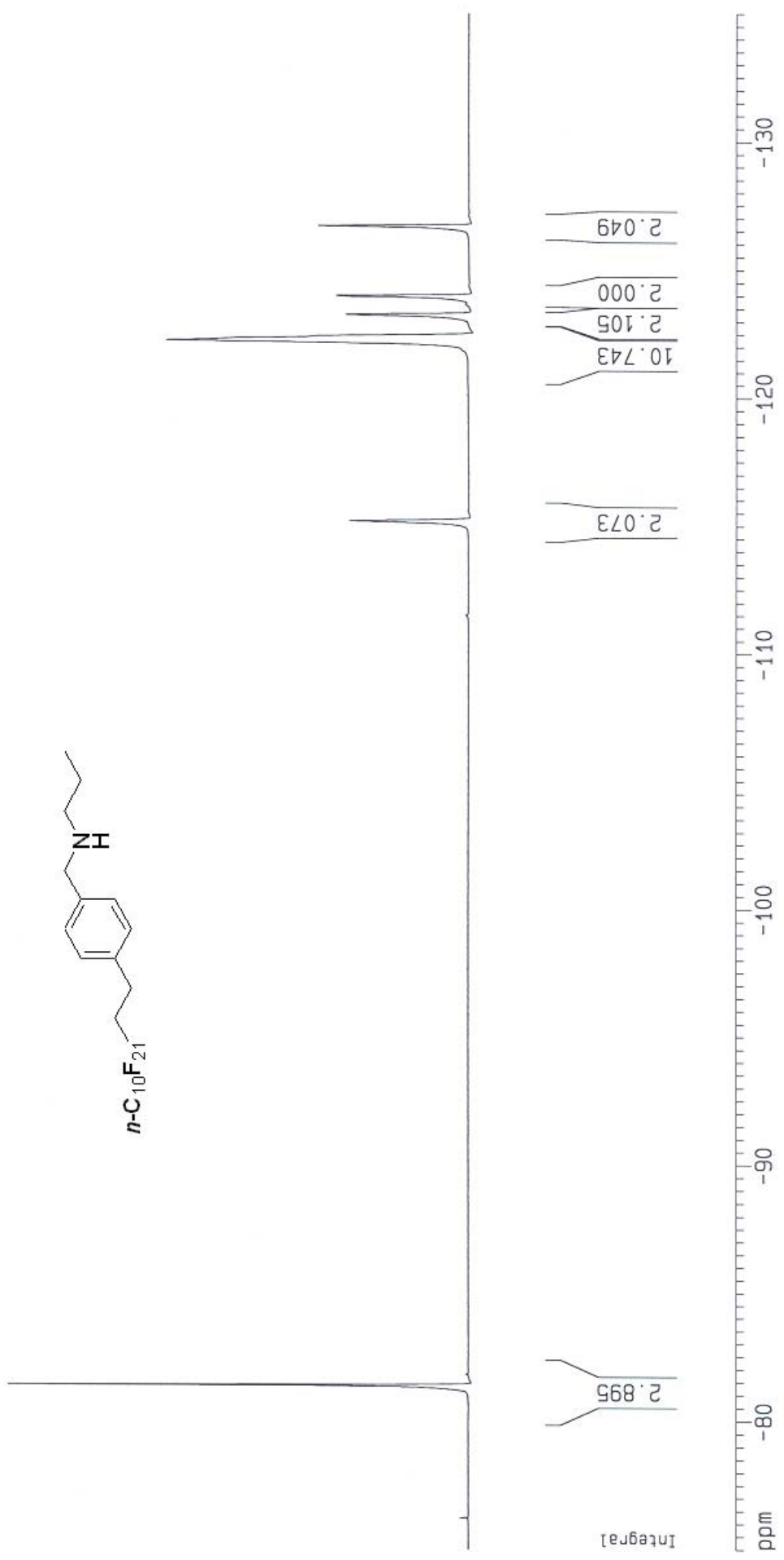

Figure S37: ${ }^{19}$ F NMR of 7d 


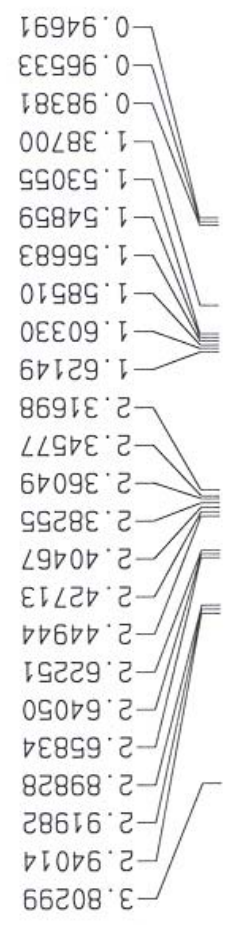

8० L8 $\angle$ $0 \angle 002 \cdot \angle \square$ EOOLE $\angle$ จटઢटE : $\angle-$

wdd

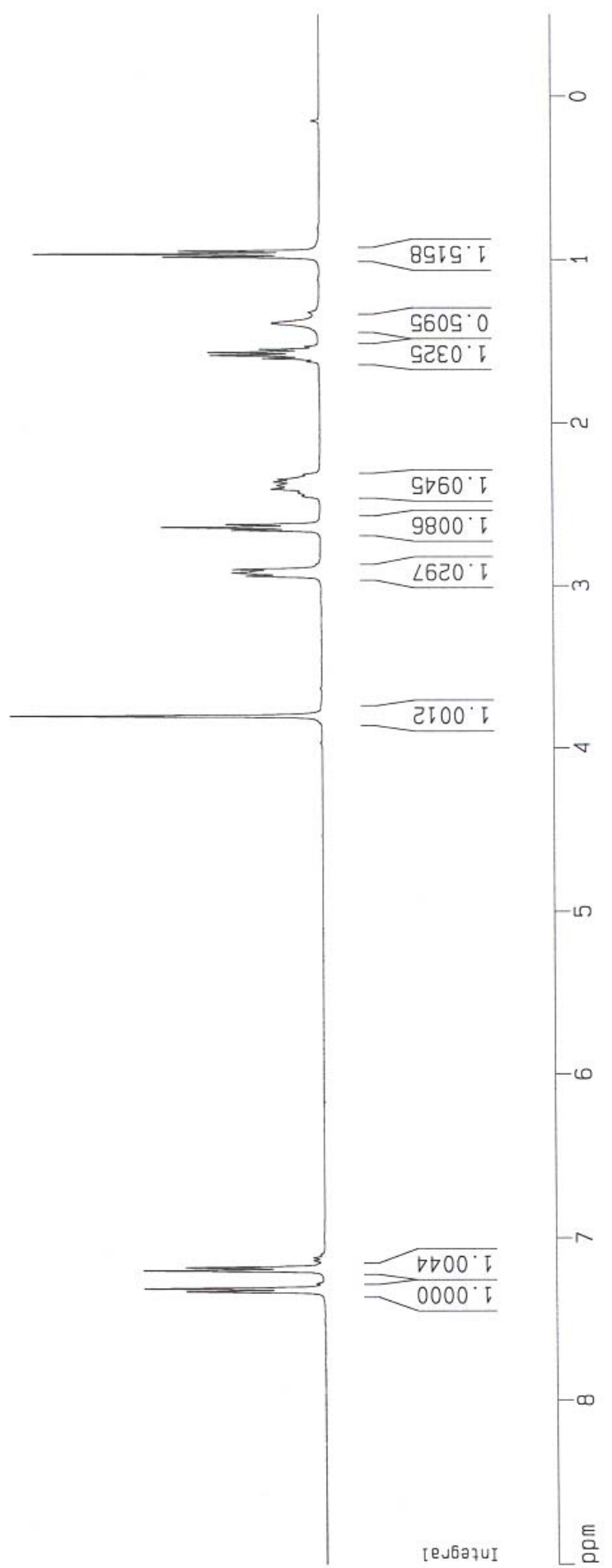

Figure S38: ${ }^{1} \mathrm{H}$ NMR of $7 \mathbf{d}$ 


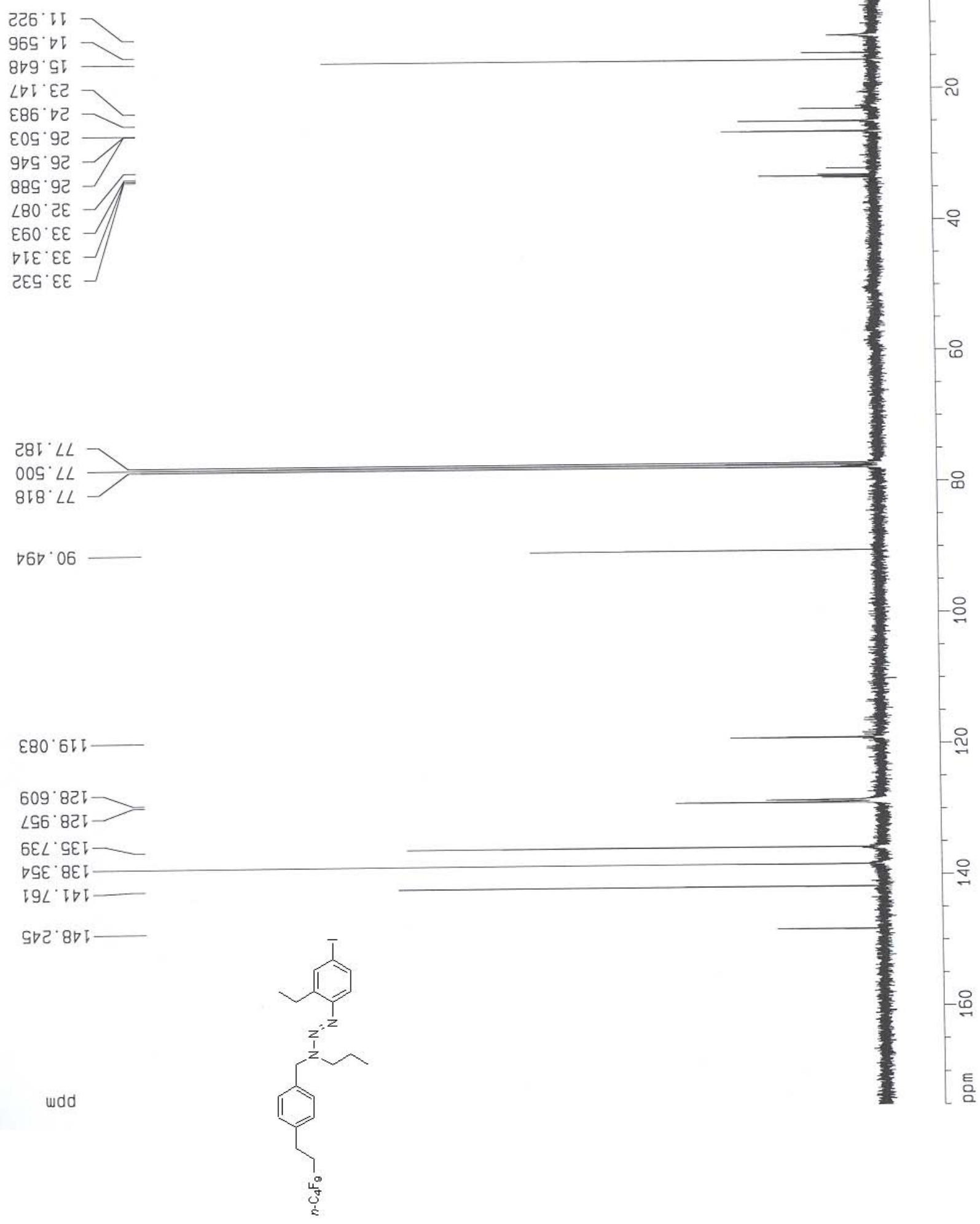

Figure S39: ${ }^{13} \mathrm{C}$ NMR of $\mathbf{8 a}$ 
Lट. פटा--

92 . $\mathrm{g} \mathrm{t}$

$\nabla ट \cdot \mathrm{g} L \mathrm{I}-$

દट. ૬टा-

ટટ' 9 ટ -

$99^{\circ} \varepsilon ट \digamma-$

ต9. $દ ट ा-$

$60^{\circ} \nabla L$

$90^{\circ} \nabla L L-$

SO० $\nabla$ L -

$\varepsilon 0^{\circ} \nabla L \leftarrow-$
$\varepsilon 0^{\circ} \nabla L-$

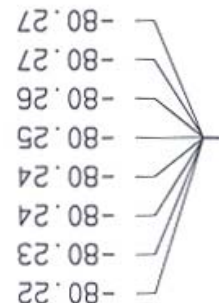

wdd

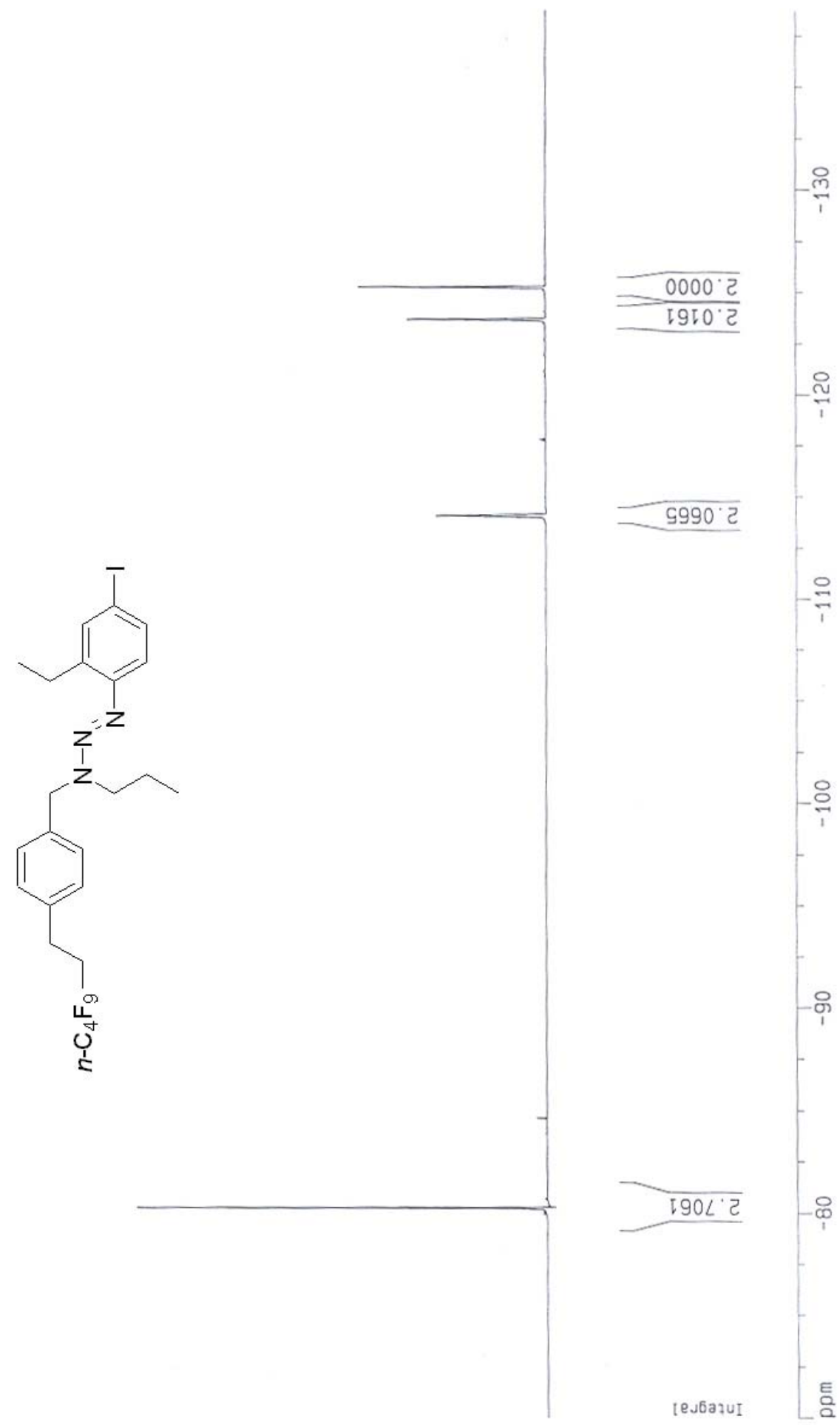

Figure S40: ${ }^{19} \mathrm{~F}$ NMR of $\mathbf{8 a}$ 

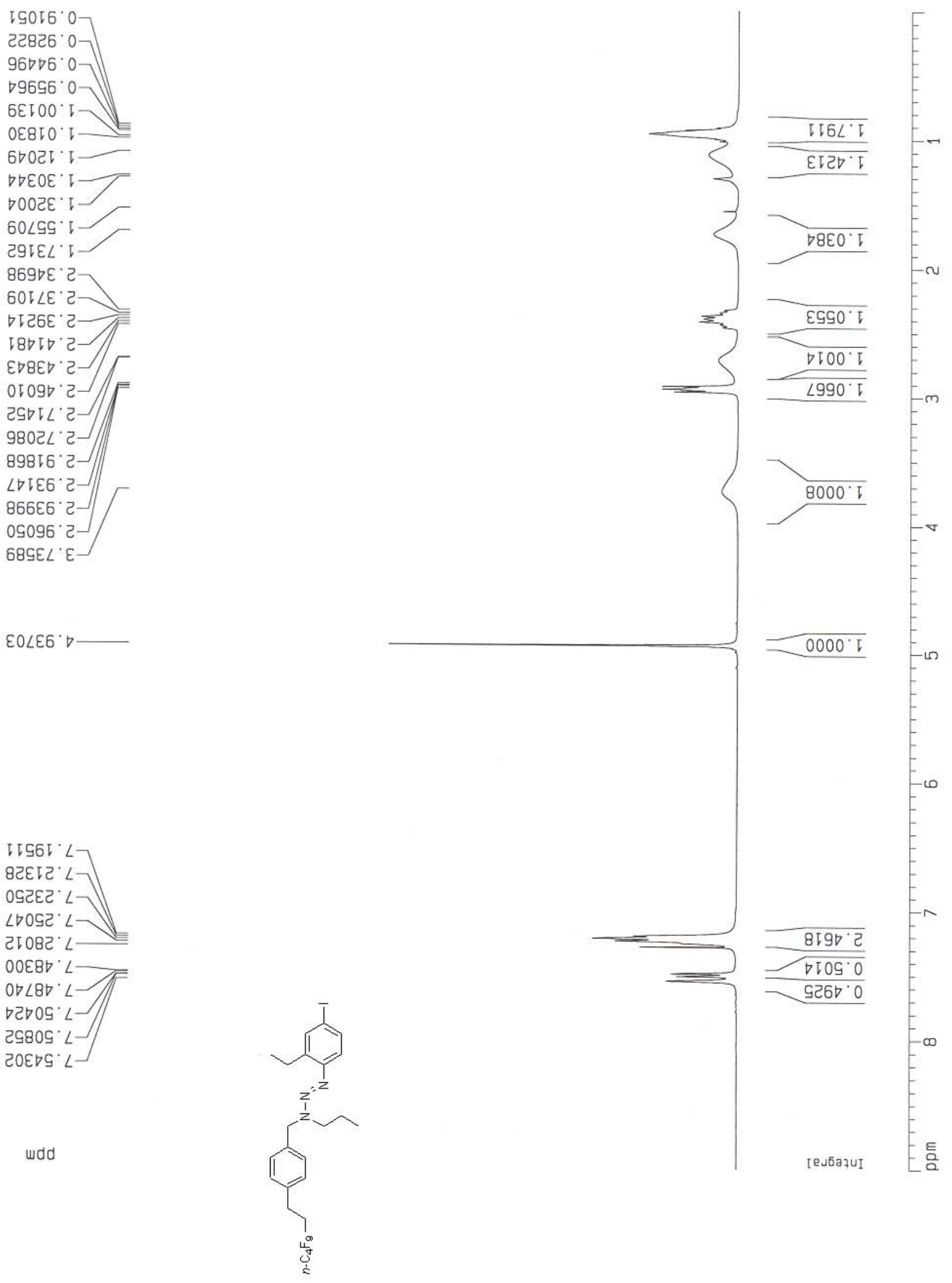

Figure S41: ${ }^{1} \mathrm{H}$ NMR of $8 \mathbf{a}$ 


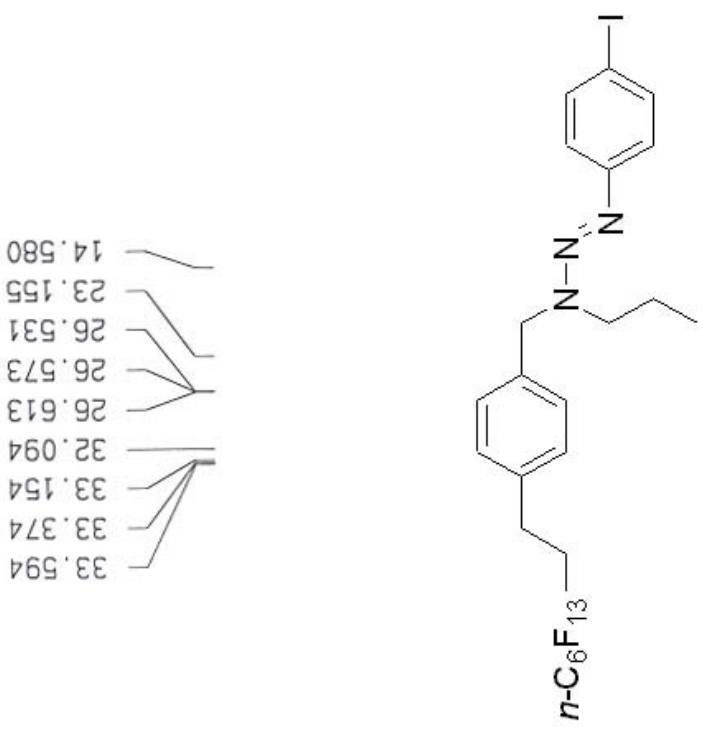

$\angle 81^{\circ} \angle L$

$\triangle O G^{\circ} \angle L$

Iट8 $\angle L$

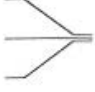

$866^{\circ} 68$

દ૬ '

$6 \angle V^{\circ} \varepsilon 己 L \longrightarrow$

0เ6 8ट I

$\varepsilon 00^{\circ} 6 \mathrm{~L}$ -

$8 \angle C^{\prime} 8 \varepsilon \downarrow$

$\subseteq \angle 6^{\circ} O S L$

wdd

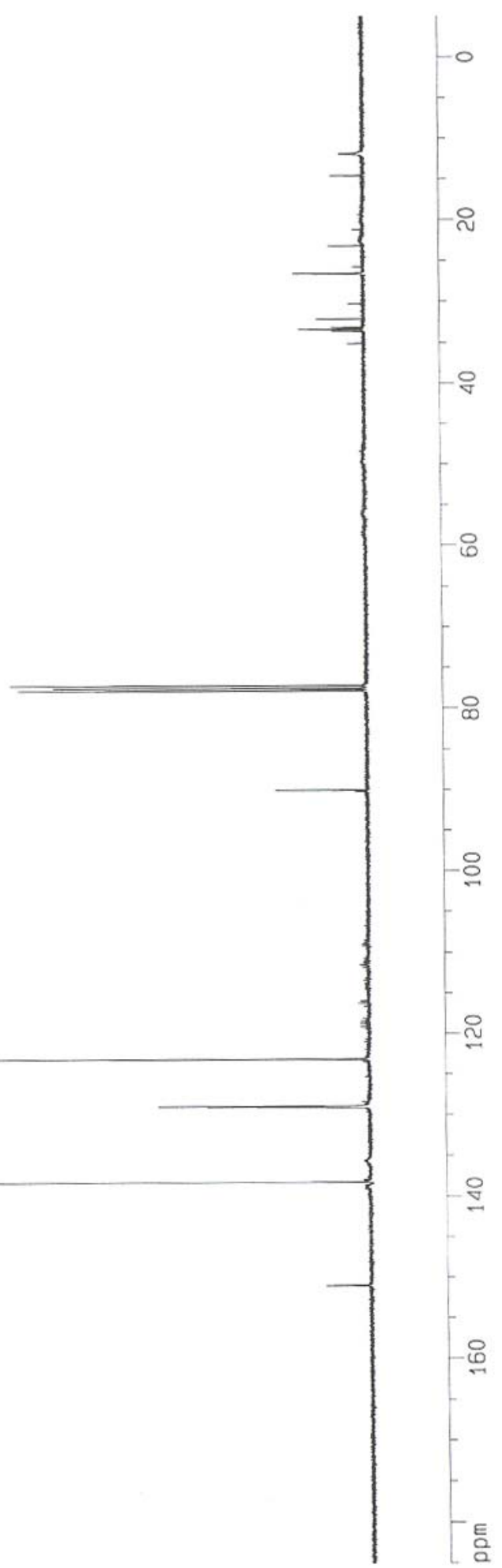

Figure S42: ${ }^{13} \mathrm{C}$ NMR of $\mathbf{8 b}$ 


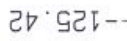

Or.

$9 L^{\circ}$ टटा-

ᄃા.

与I. Iटा--

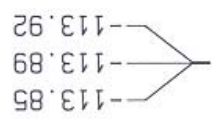

Or $08-$

wdd

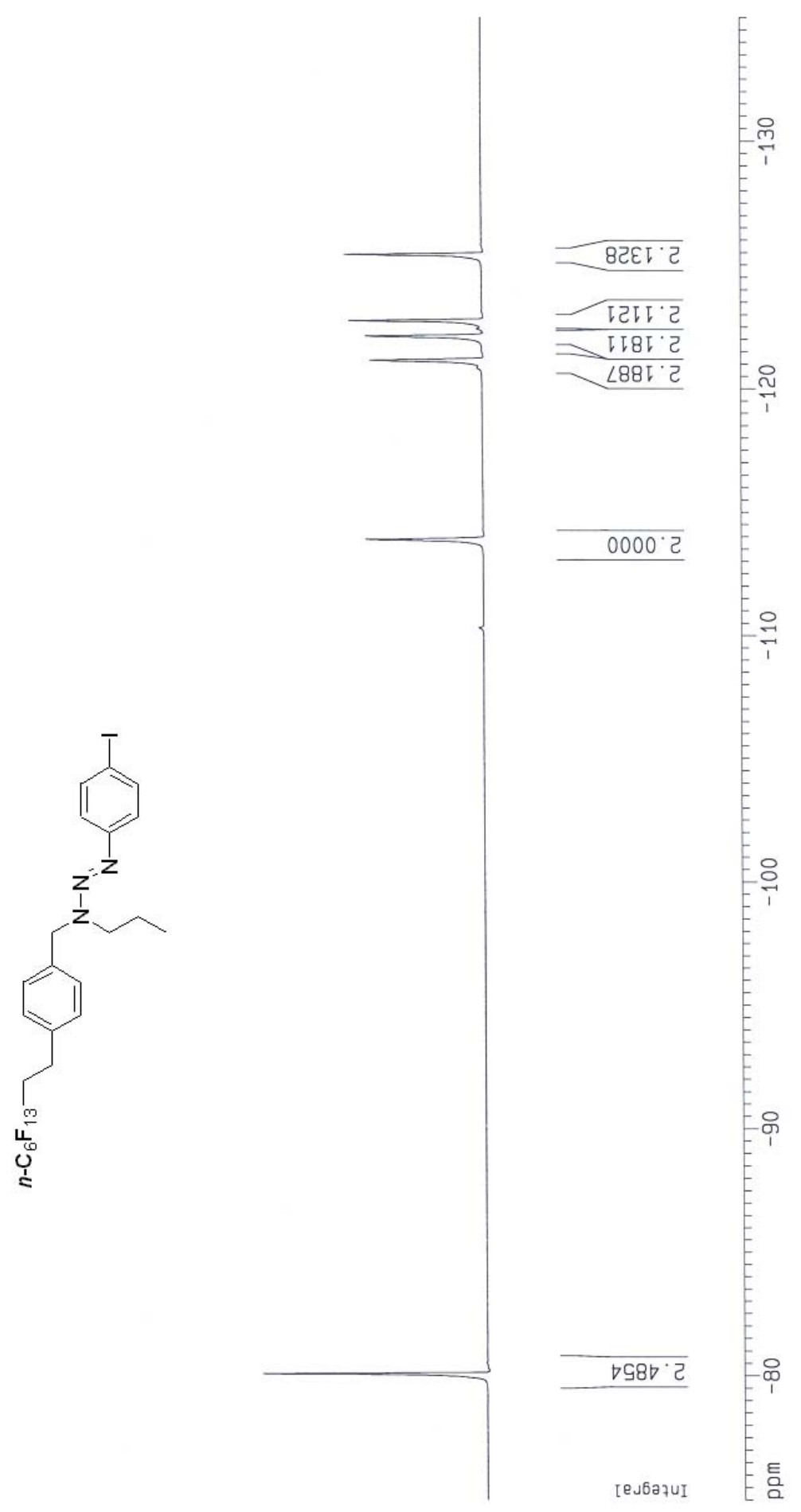

Figure S43: ${ }^{19}$ F NMR of $\mathbf{8 b}$ 

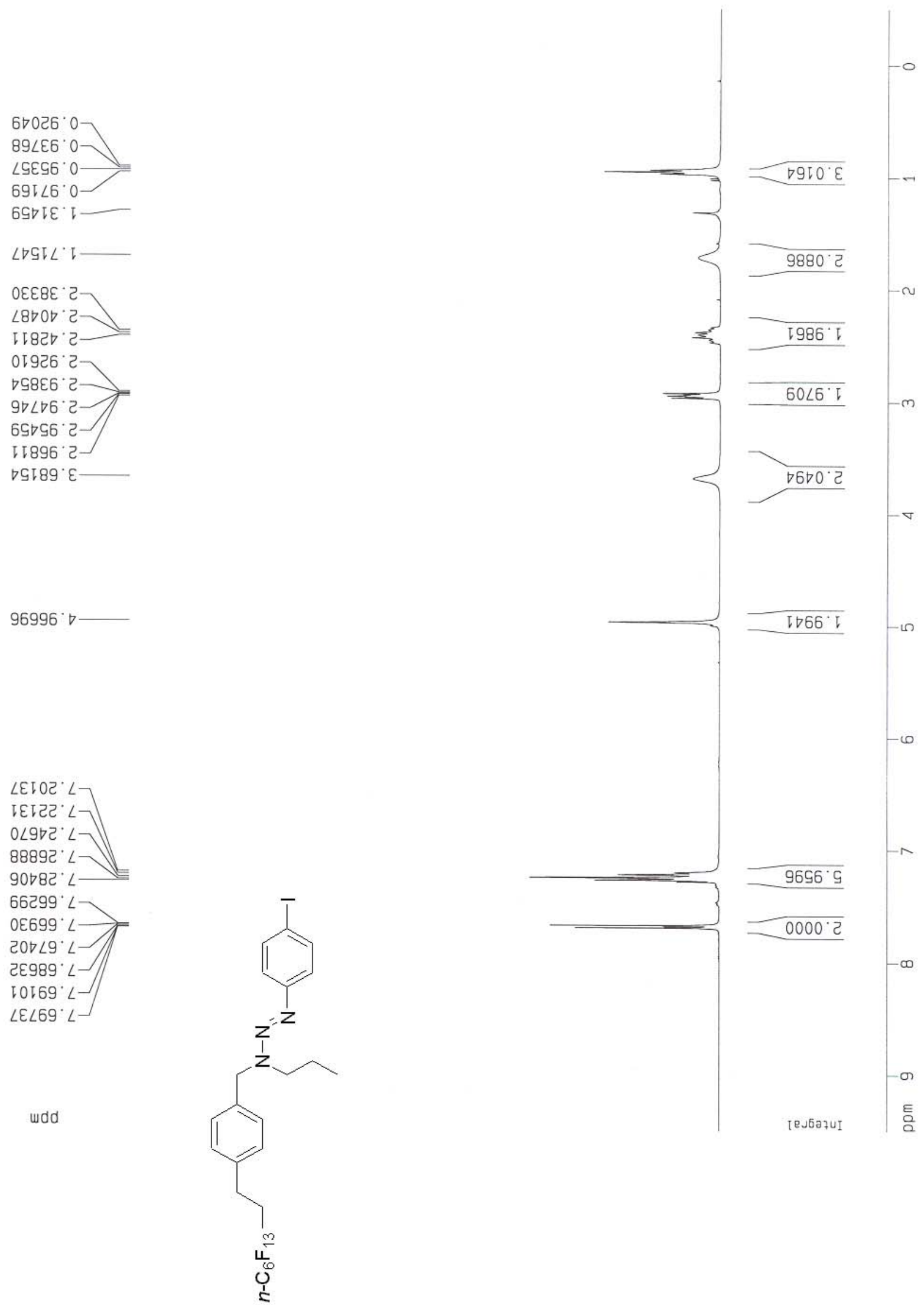

Figure S44: ${ }^{1}$ H NMR of $\mathbf{8 b}$ 


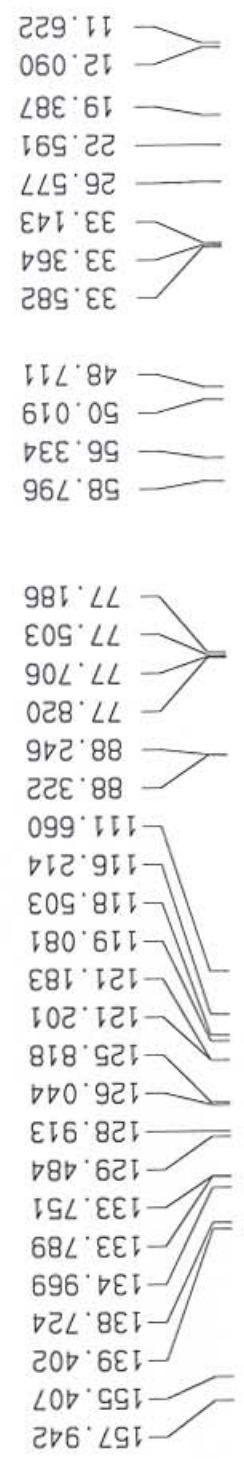

wdd

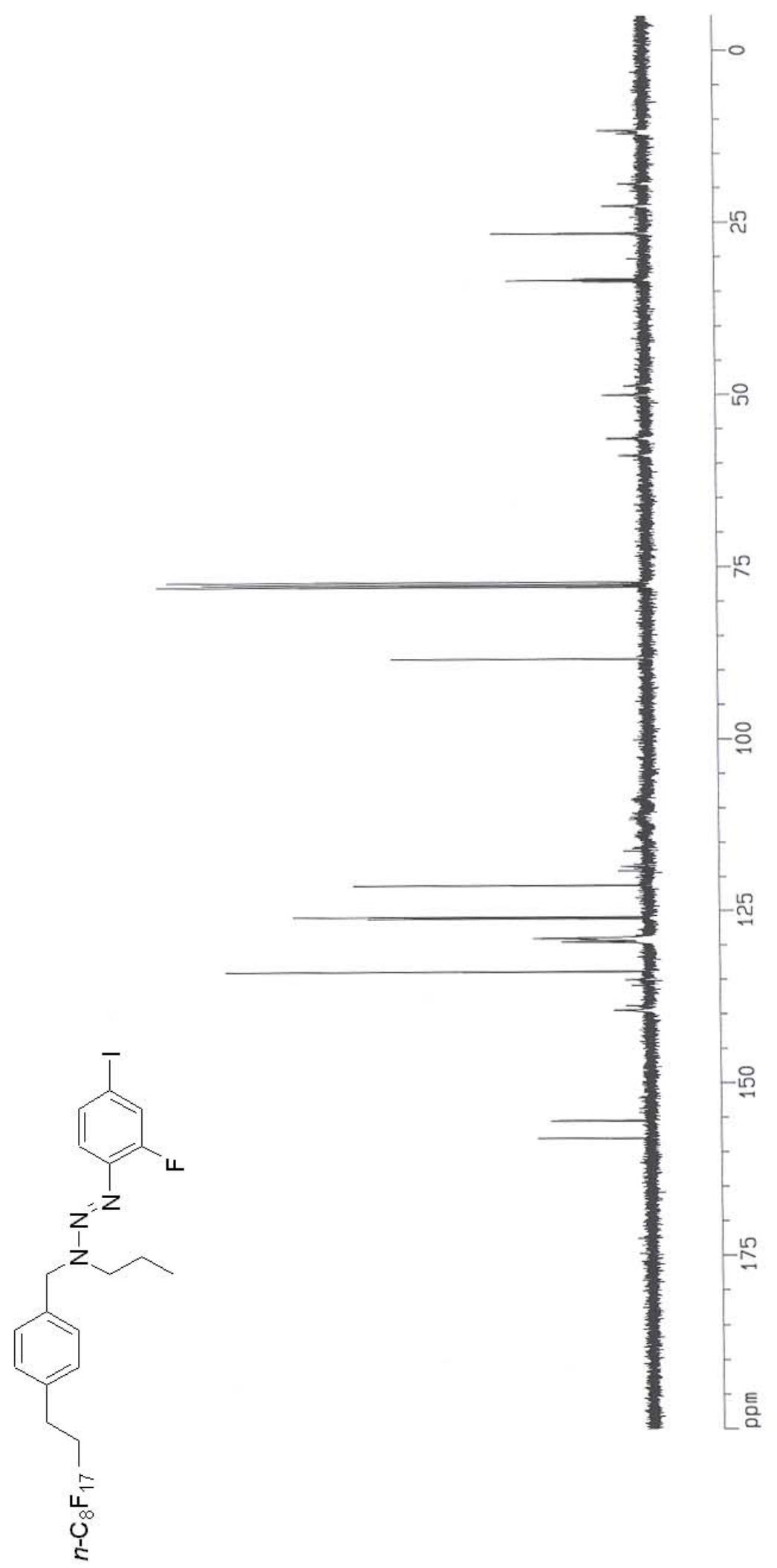

Figure S45: ${ }^{13} \mathrm{C}$ NMR of $\mathbf{8 c}$ 

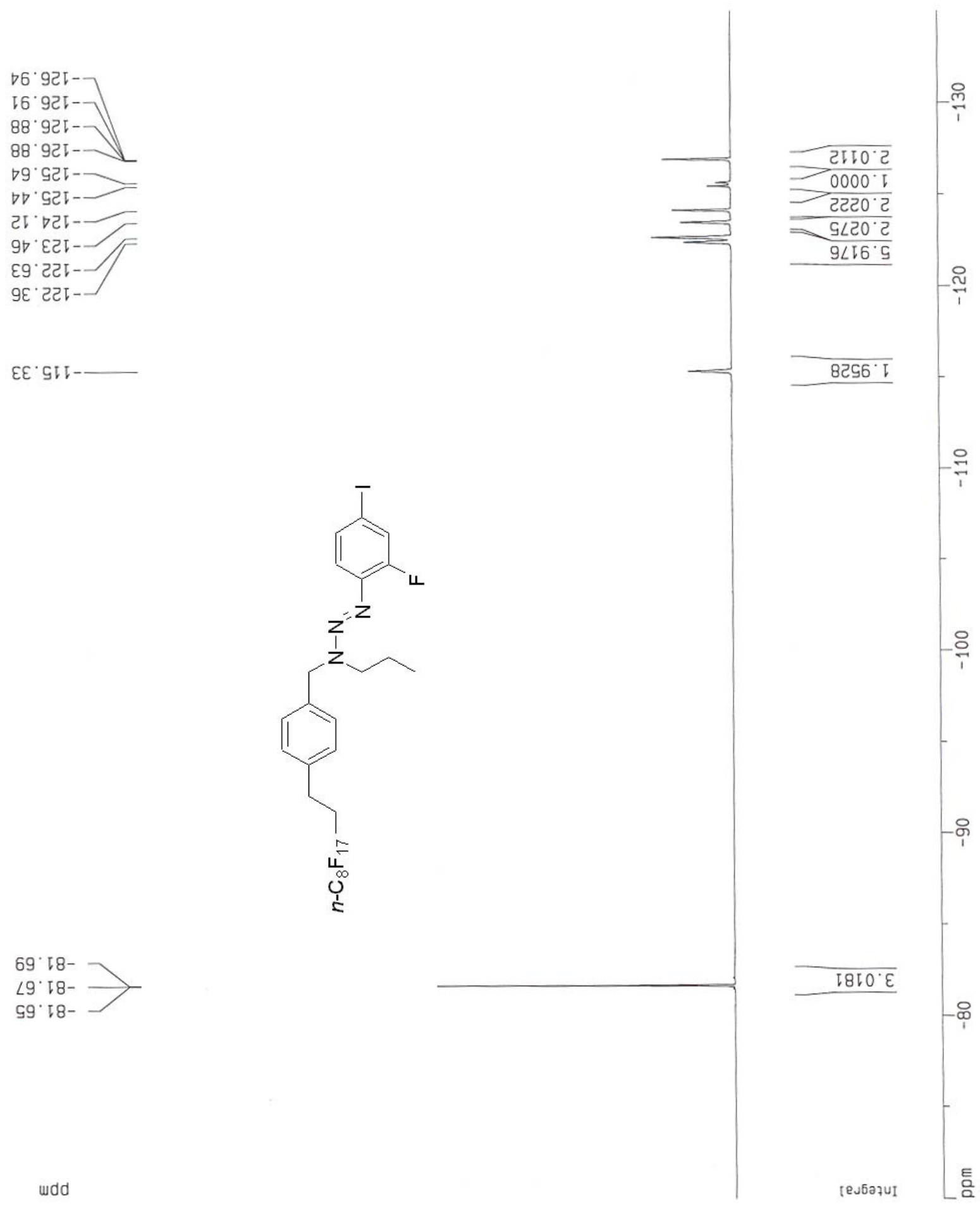

$69^{\circ}$. $18->$

99. เ8-

wdd

Figure S46: ${ }^{19}$ F NMR of $\mathbf{8 c}$ 

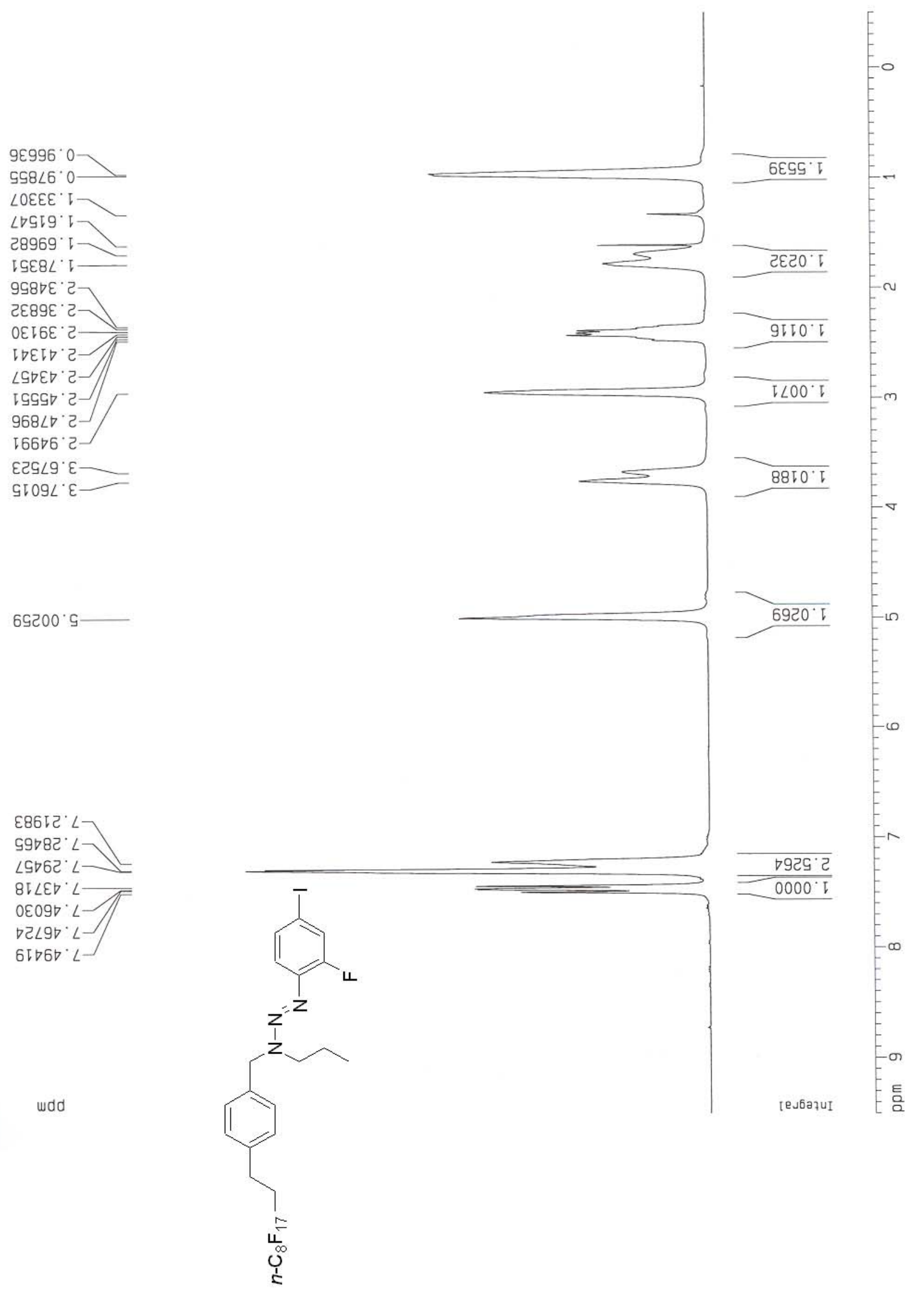

wdd

Figure S47: ${ }^{1} \mathrm{H}$ NMR of $\mathbf{8 c}$ 


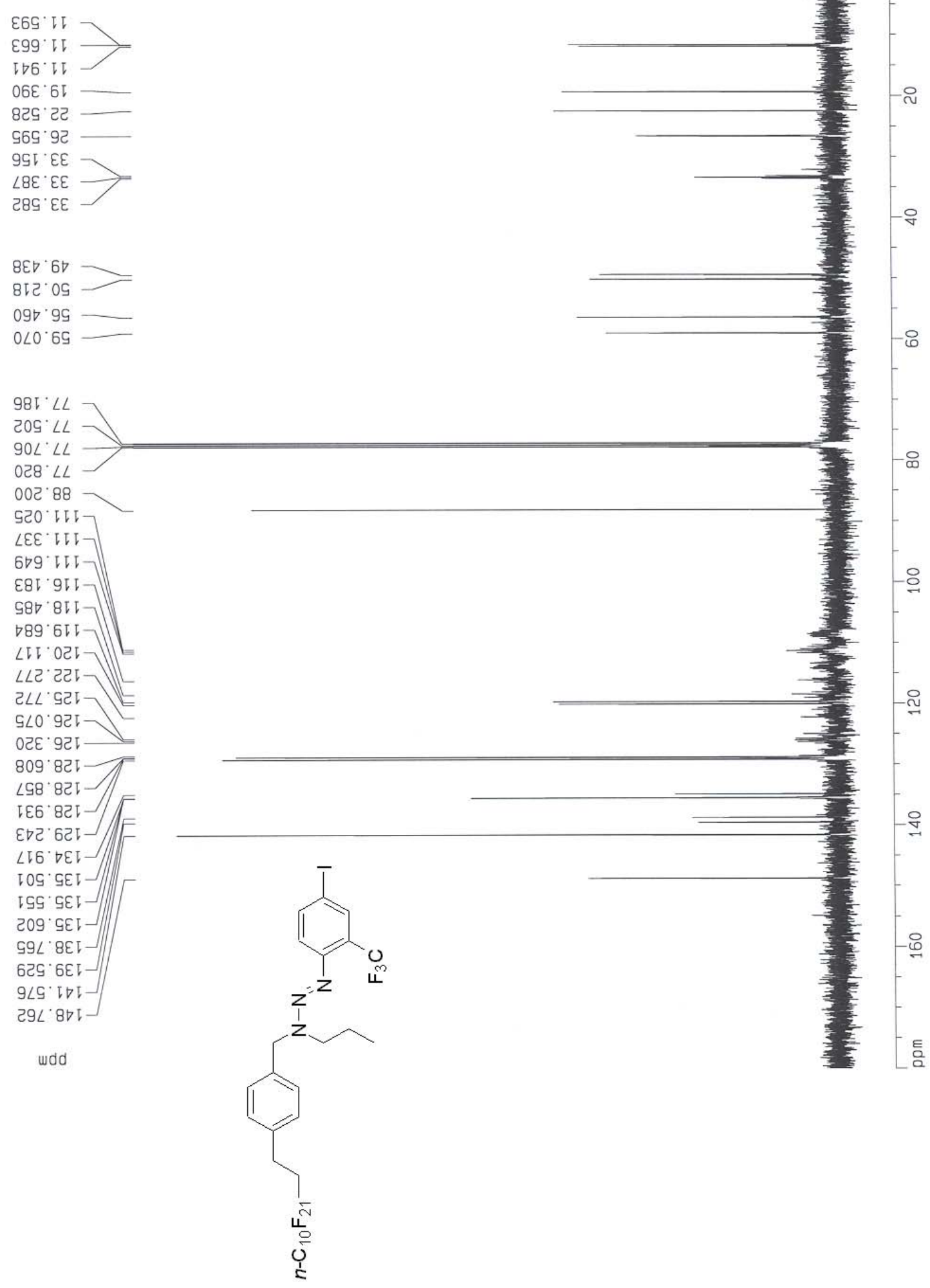

Figure S48: ${ }^{13} \mathrm{C}$ NMR of $\mathbf{8 d}$ 


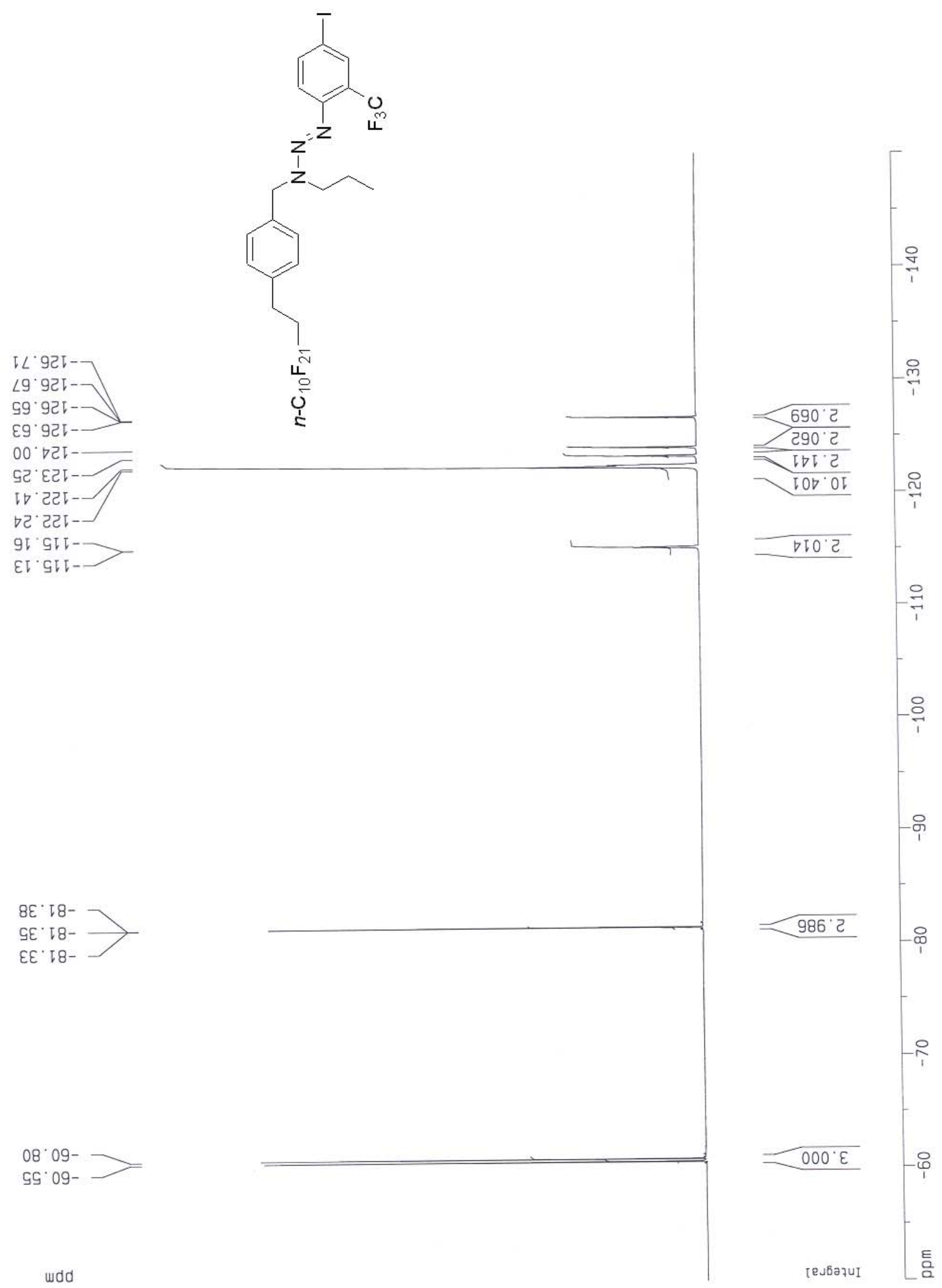

Figure S49: ${ }^{19} \mathrm{~F}$ NMR of $\mathbf{8 d}$ 

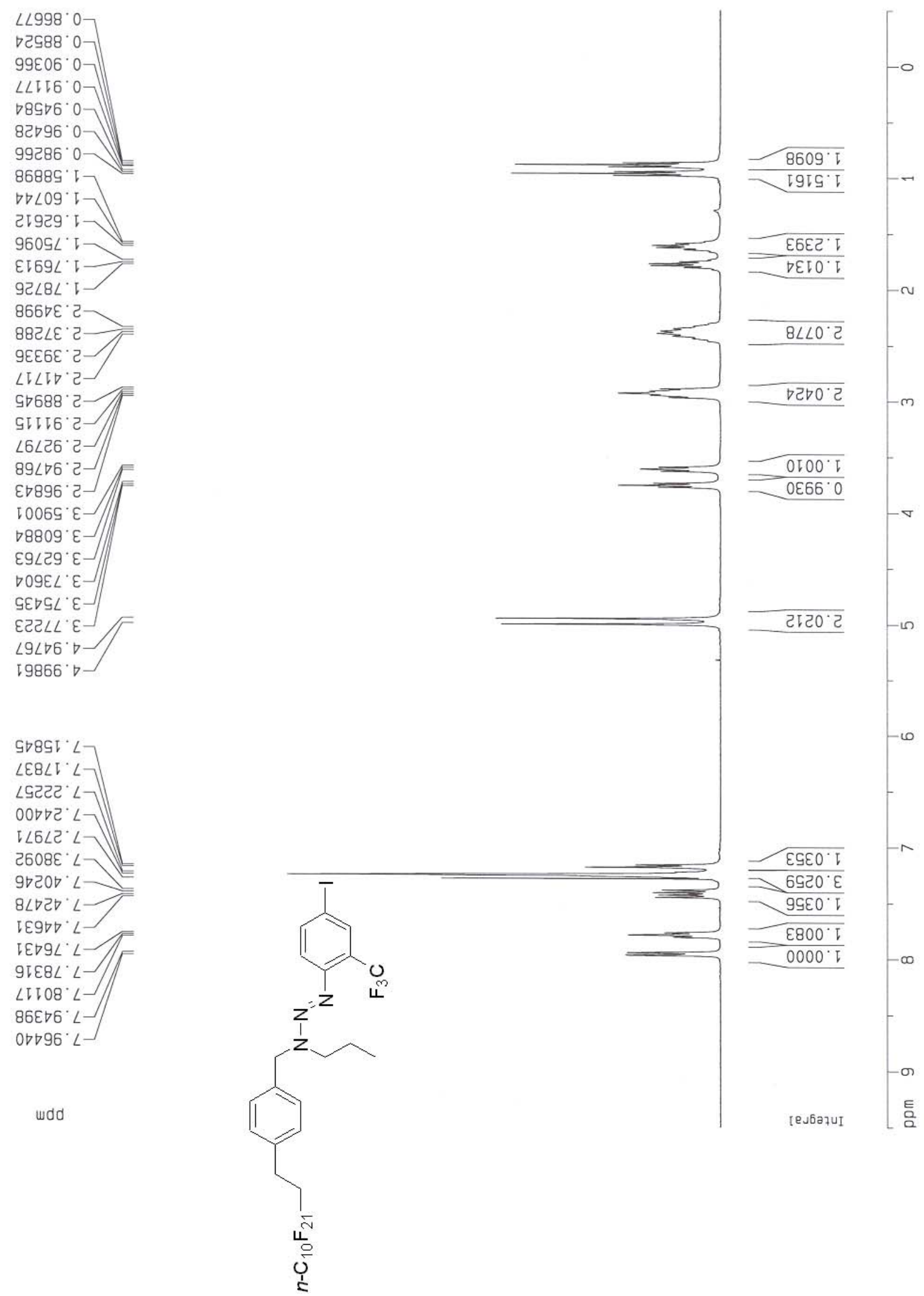

Figure S50: ${ }^{1} \mathrm{H}$ NMR of $\mathbf{8 d}$ 
$\varepsilon 69^{\circ} \nabla V$

โ9G. 95

$\angle 00^{\circ} \mathrm{G} 2$

I98 $E$ E

$08 I^{\circ} L L$

$660^{\circ} \angle L$

$\angle$ I8 $\angle L$

$090^{\circ} 60 \mathrm{~L}$

$\angle E 6^{\circ} O E \amalg-$

ᄂ I8. EE -

GSD IDI

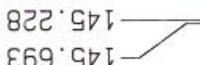

060.265

udd

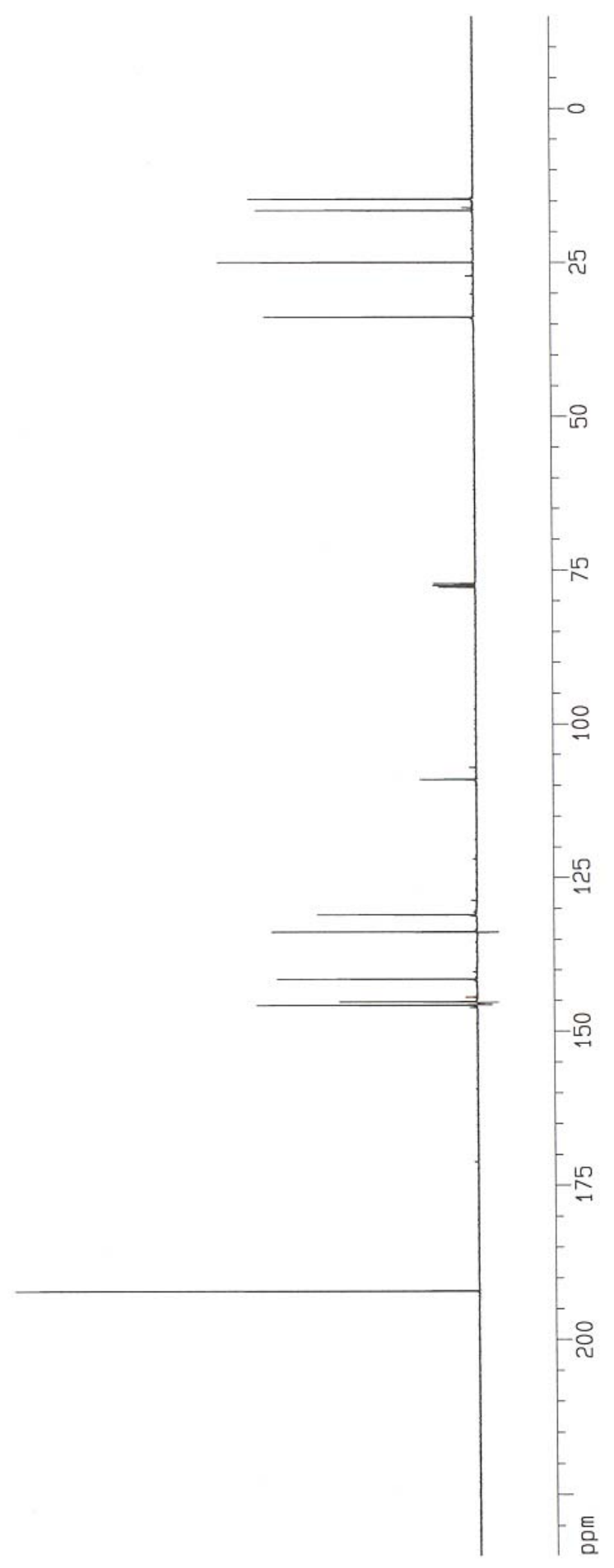

Figure S51: ${ }^{13} \mathrm{C}$ NMR of $\mathbf{1 1}$ 


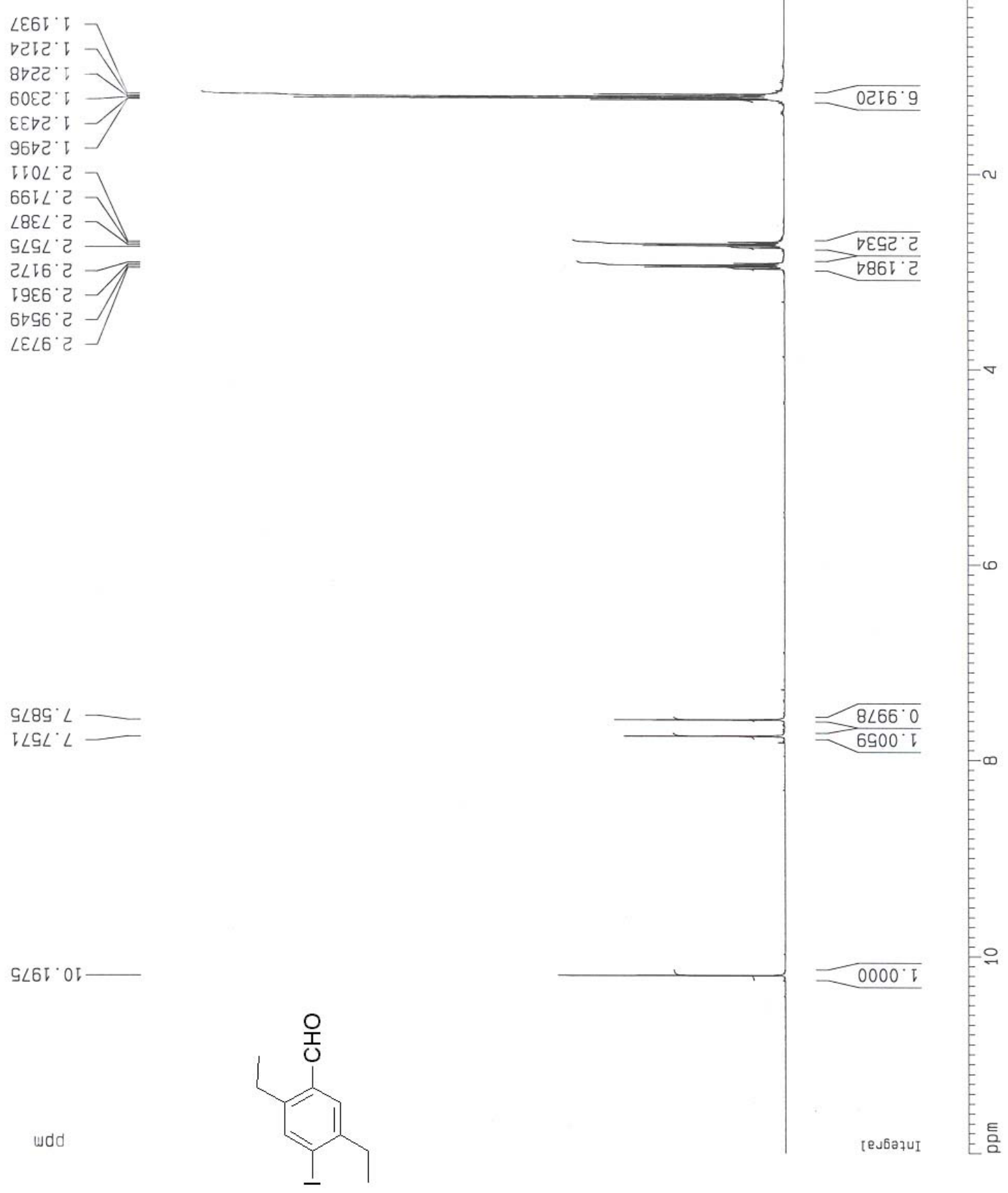

Figure S52: ${ }^{1} \mathrm{H}$ NMR of $\mathbf{1 1}$ 


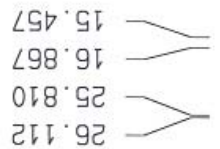

$6 \angle V^{\circ} \angle L$ $\angle 6 \nabla^{\circ} \angle L$ $\triangle 18{ }^{\circ} \angle L$

$\varepsilon \varepsilon \nabla^{\circ} 8 \tau \longrightarrow$ $\angle E 8^{\circ} \angle 2 T$ 9SD $2 E L-$ $\checkmark 6 I^{\circ} E E$ E9I $\nabla E L-$ 9LO OD $960^{\circ} \mathrm{Z} \nabla \downarrow$ $\varepsilon 66^{\circ} \nabla \nabla \downarrow-$

૬9દ' ट6 wdd<smiles>C=Cc1cc(CC)c([O-])cc1CC</smiles>

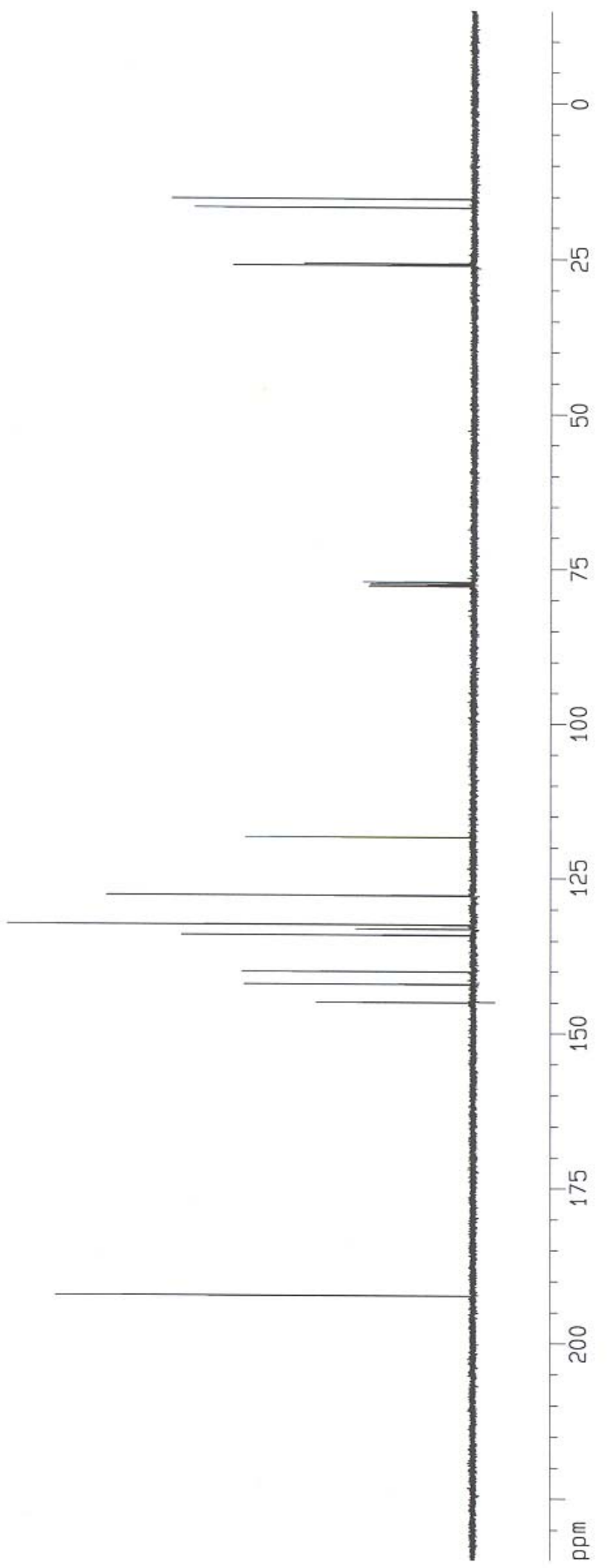

Figure S53: ${ }^{13} \mathrm{C}$ NMR of $\mathbf{1 2}$ 


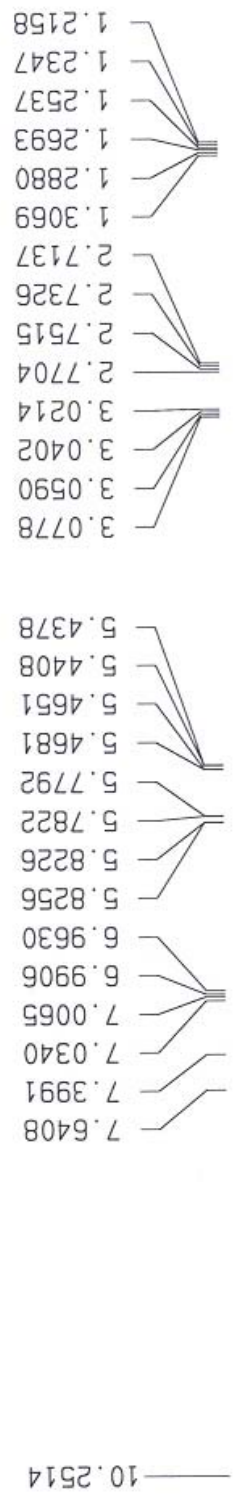

$\nabla$ ISE・0I

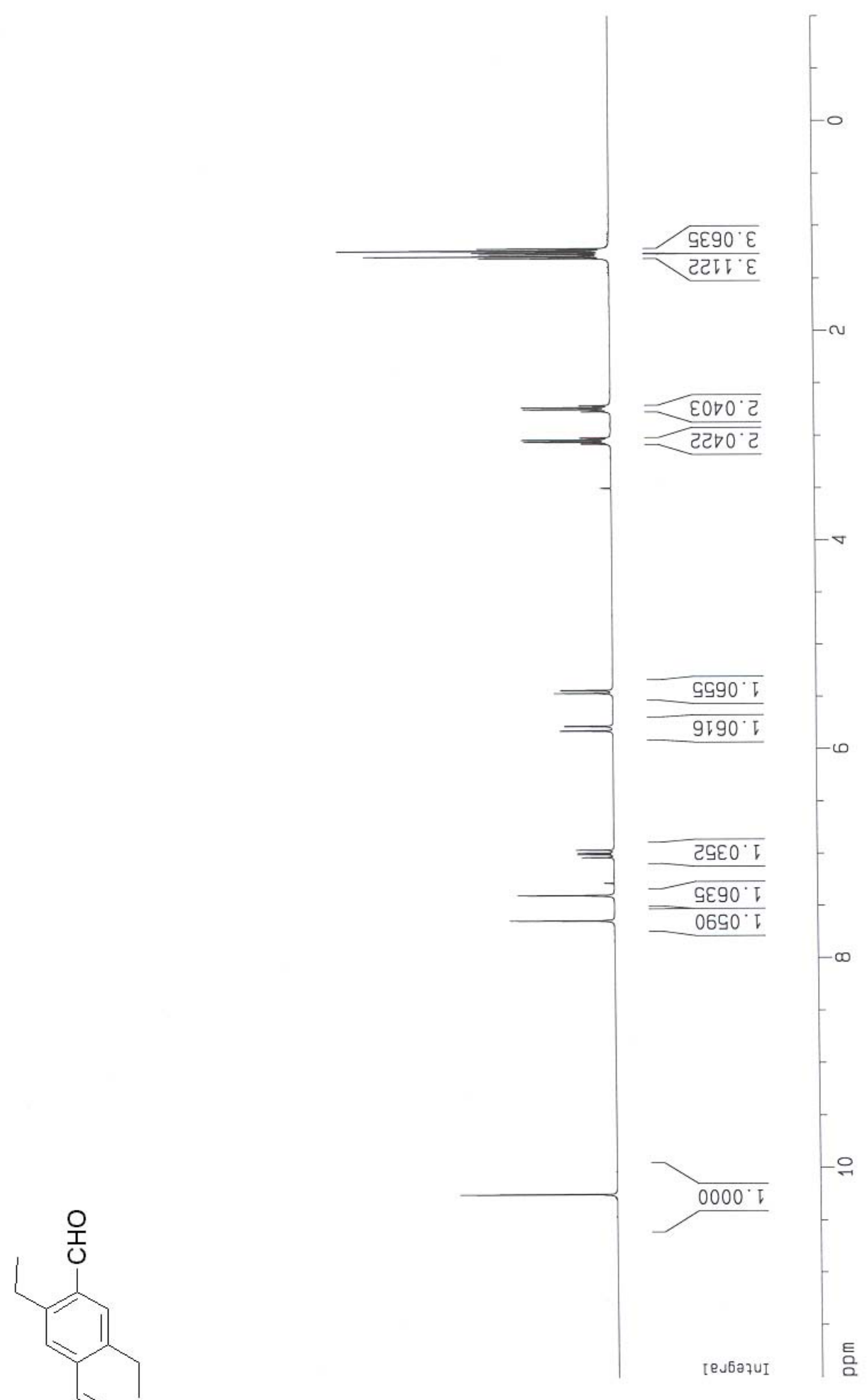

Figure S54: ${ }^{1} \mathrm{H}$ NMR of $\mathbf{1 2}$ 

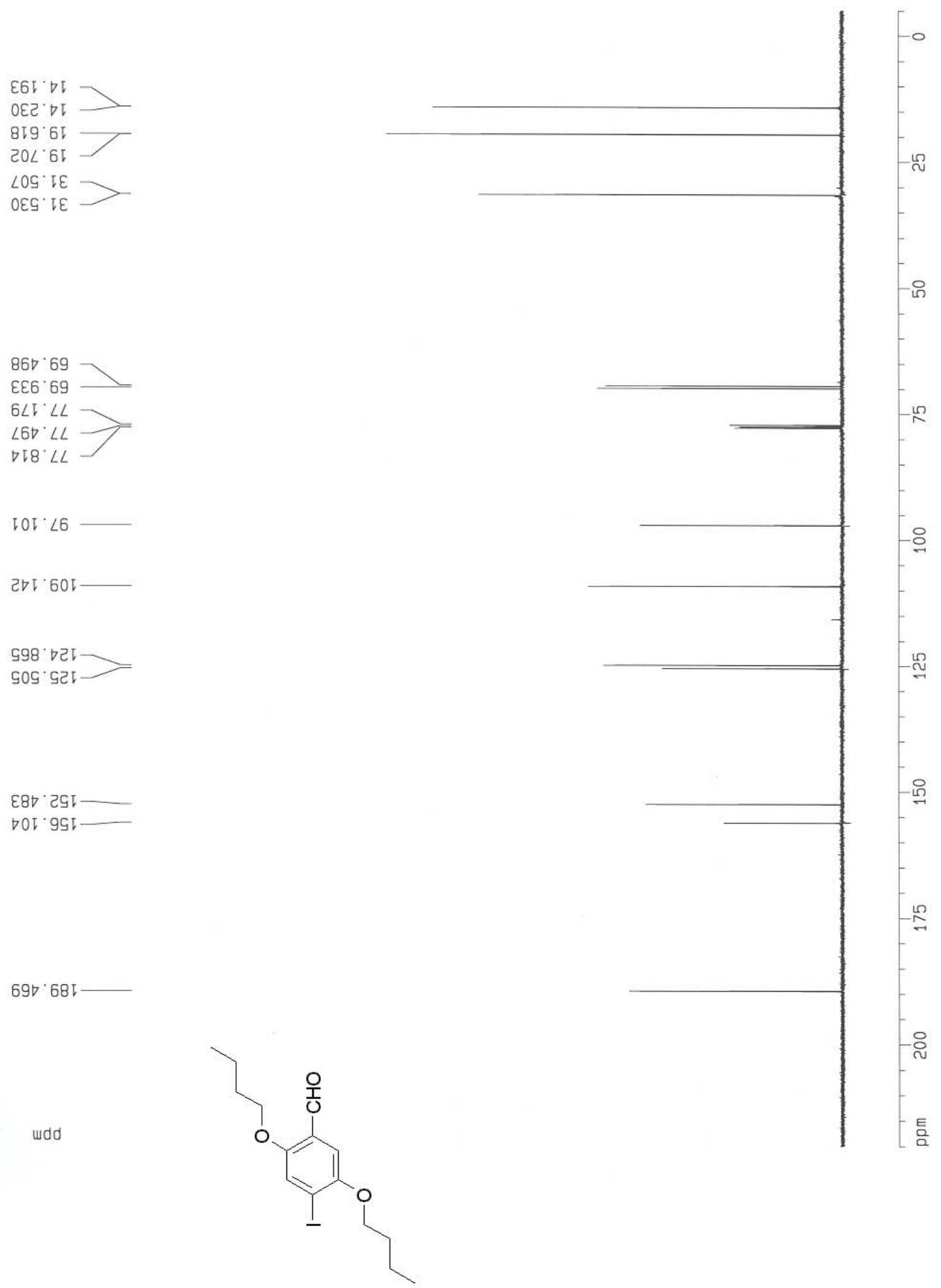

Figure S55: ${ }^{13} \mathrm{C}$ NMR of $\mathbf{1 5}$ 


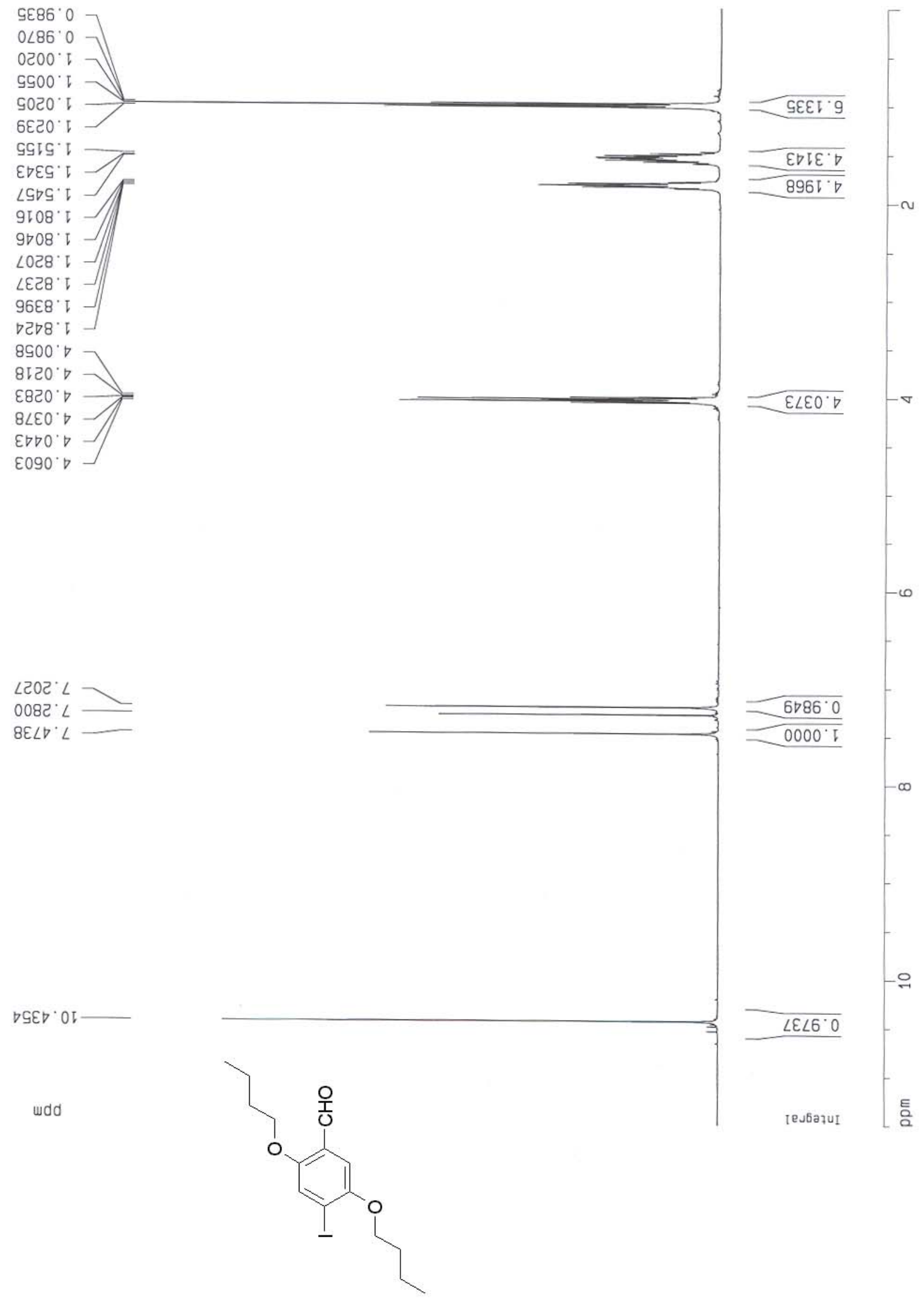

Figure S56: ${ }^{1} \mathrm{H}$ NMR of $\mathbf{1 5}$ 

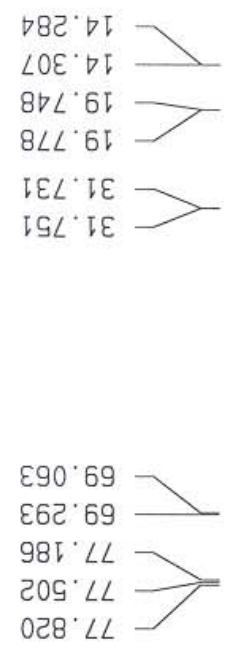

द८E. OL

Eटટ ट96 $\angle \downarrow \downarrow$

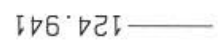

$98 L^{\circ}$ VEᄂ Gट8. $\nabla \varepsilon \downarrow$

$\nabla \nabla 6^{\circ}$ OS —

टदS 991

IL 68 I

wdd

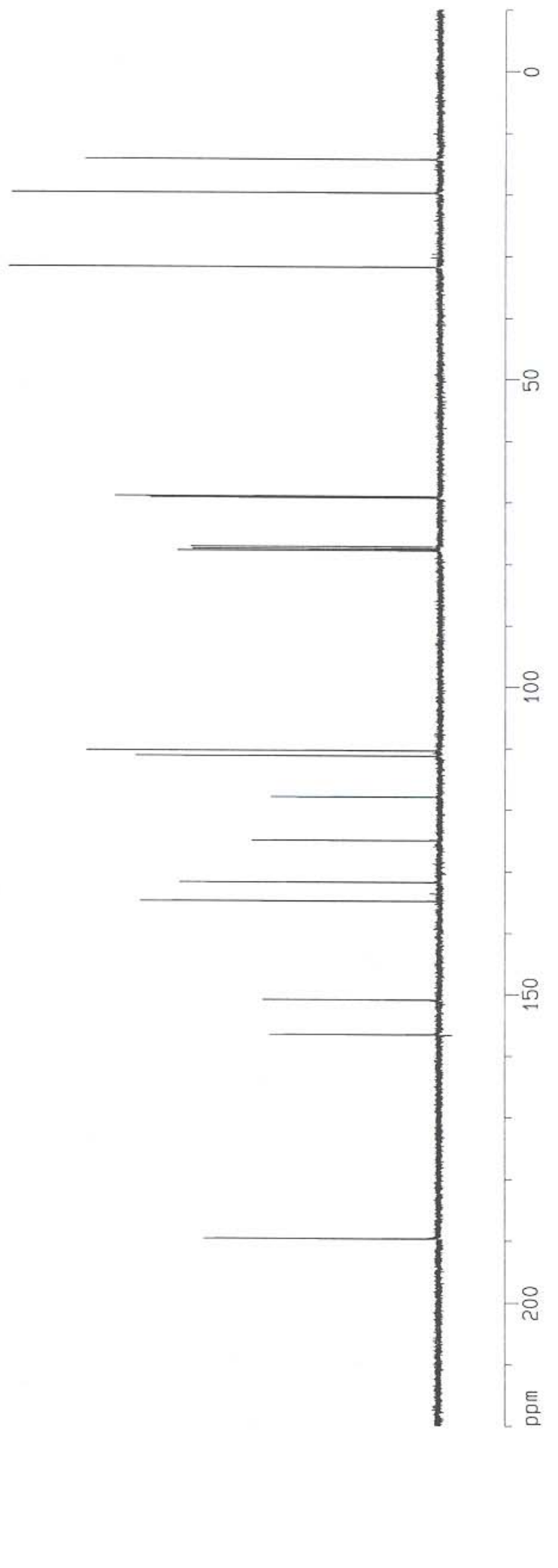

Figure S57: ${ }^{13} \mathrm{C}$ NMR of $\mathbf{1 6}$ 

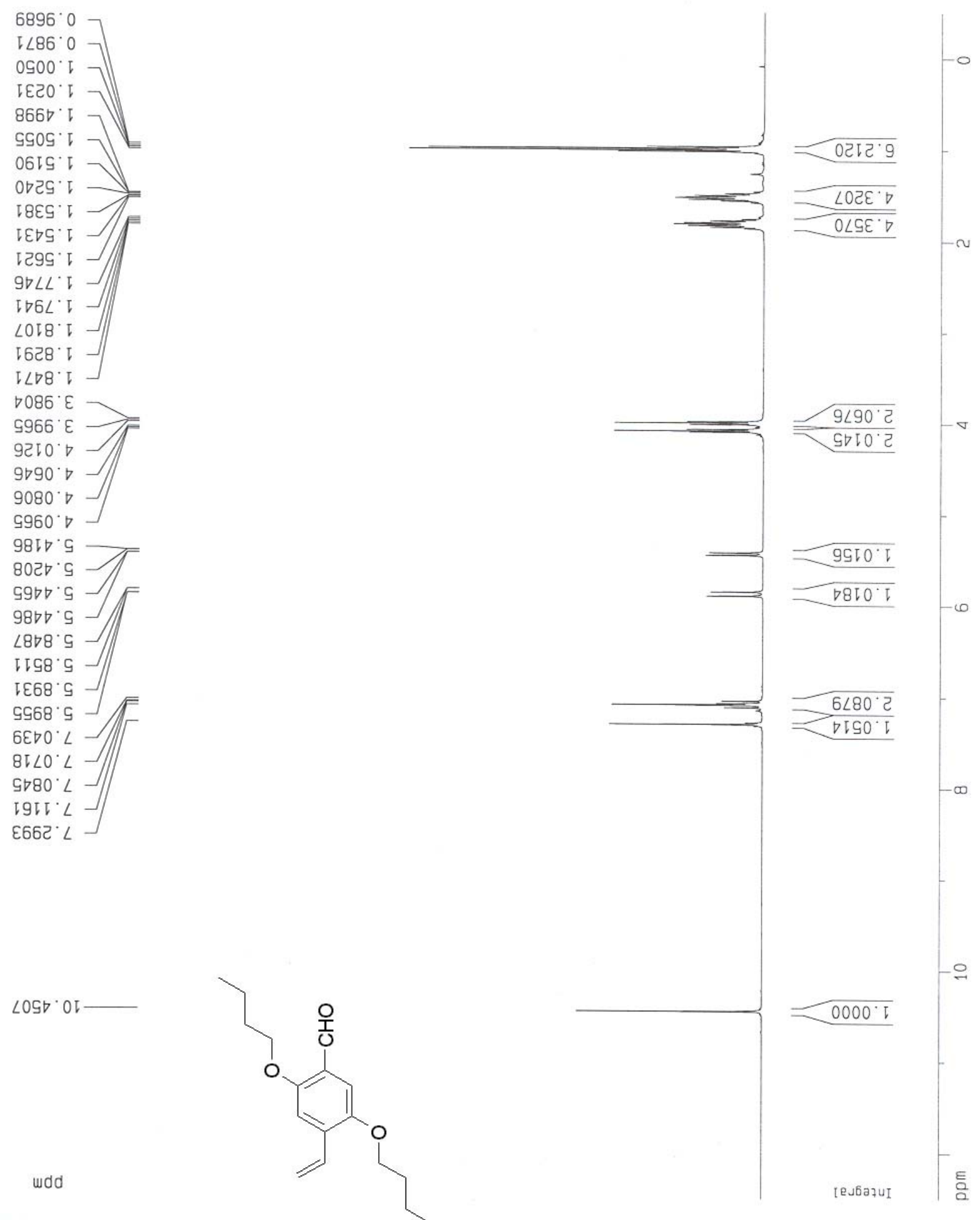

Figure S58: ${ }^{1} \mathrm{H}$ NMR of $\mathbf{1 6}$ 


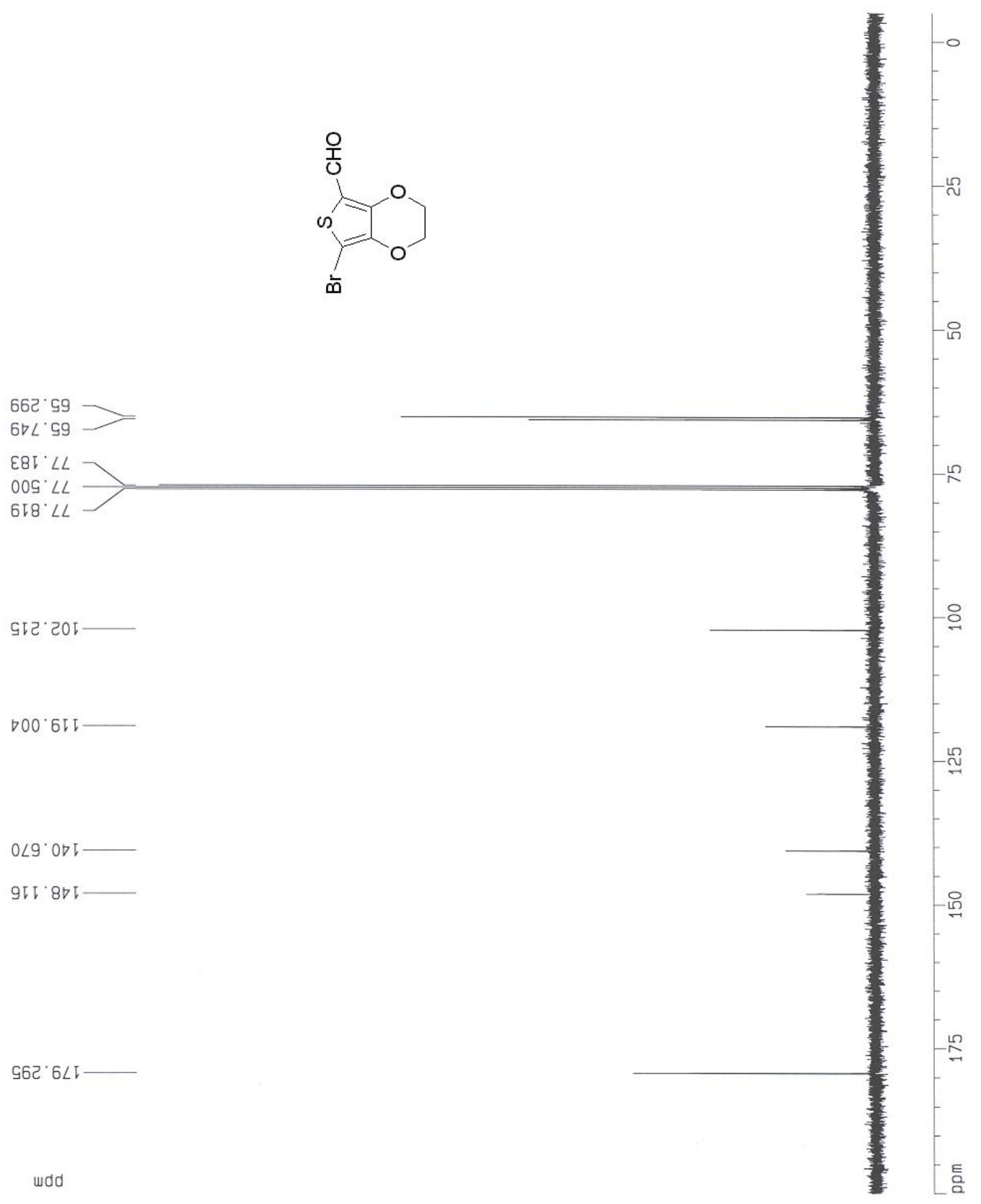

Figure S59: ${ }^{13} \mathrm{C}$ NMR of $\mathbf{1 8}$ 
$O D \angle 19^{\circ}$
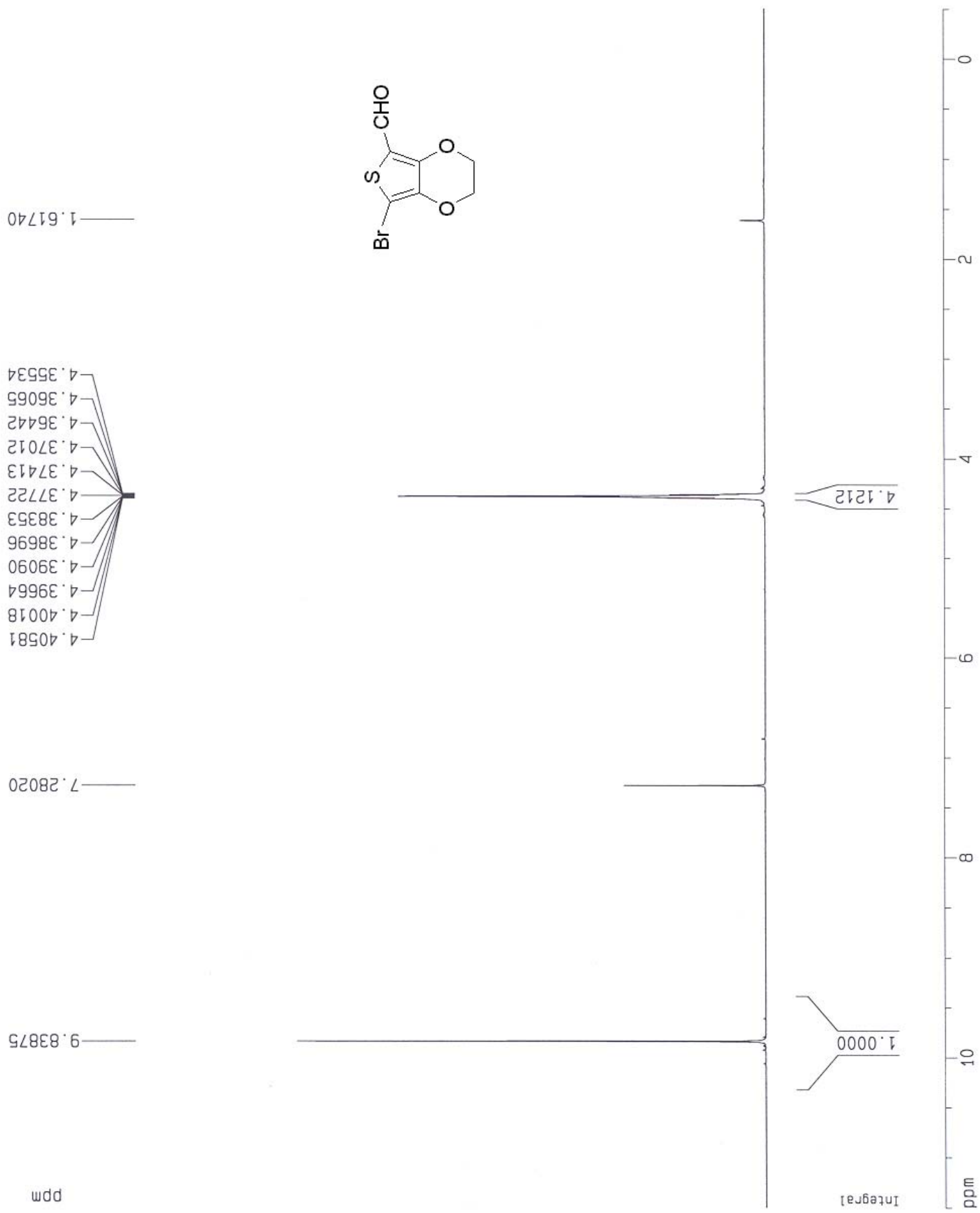

$02082^{\circ} L$

$9 \angle 8 \varepsilon 8^{\circ} 6$

wdd

Figure S60: ${ }^{1} \mathrm{H}$ NMR of $\mathbf{1 8}$ 


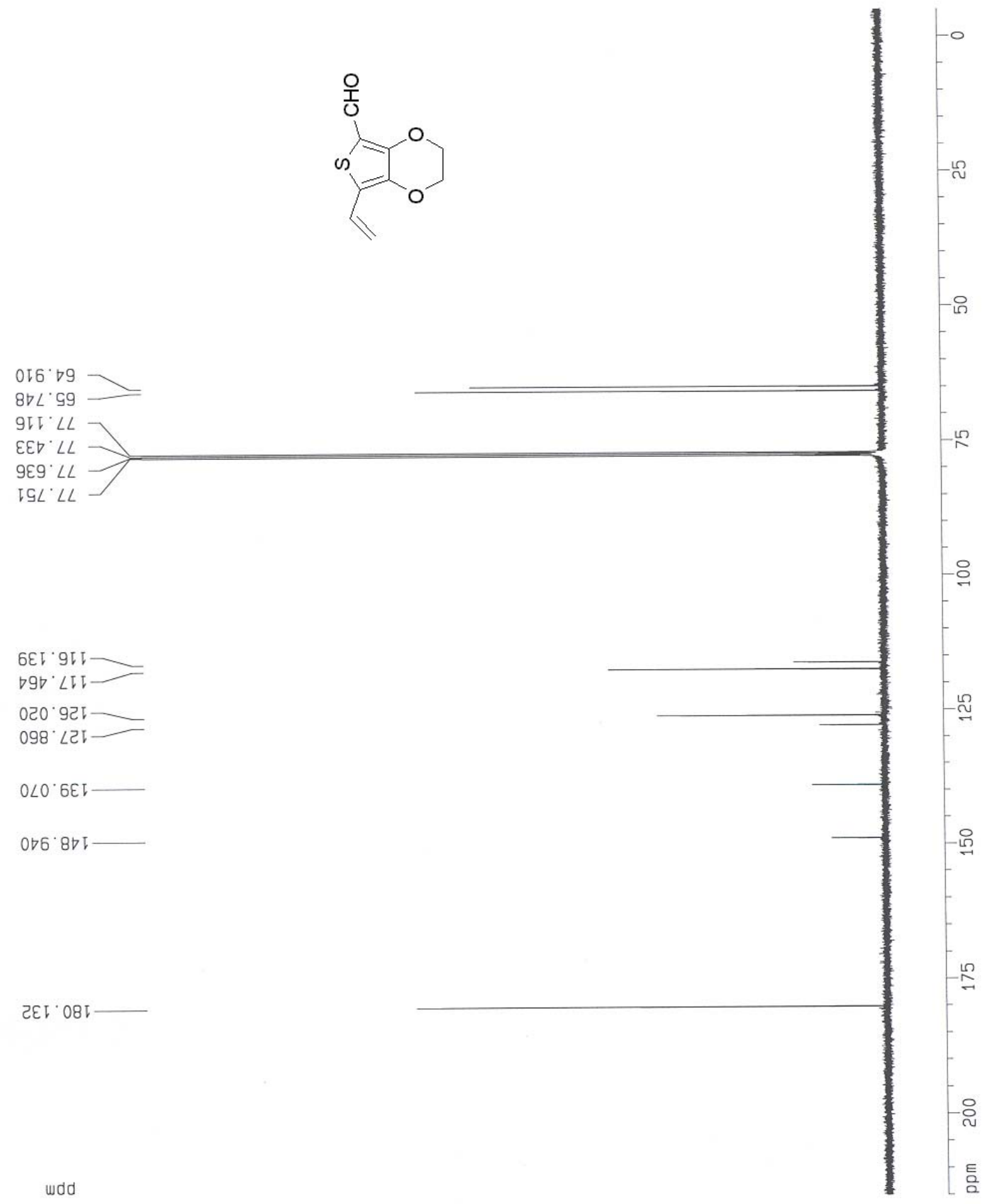

Figure S61: ${ }^{13} \mathrm{C}$ NMR of 19 


\section{$2 \angle 809^{\circ} \downarrow$}
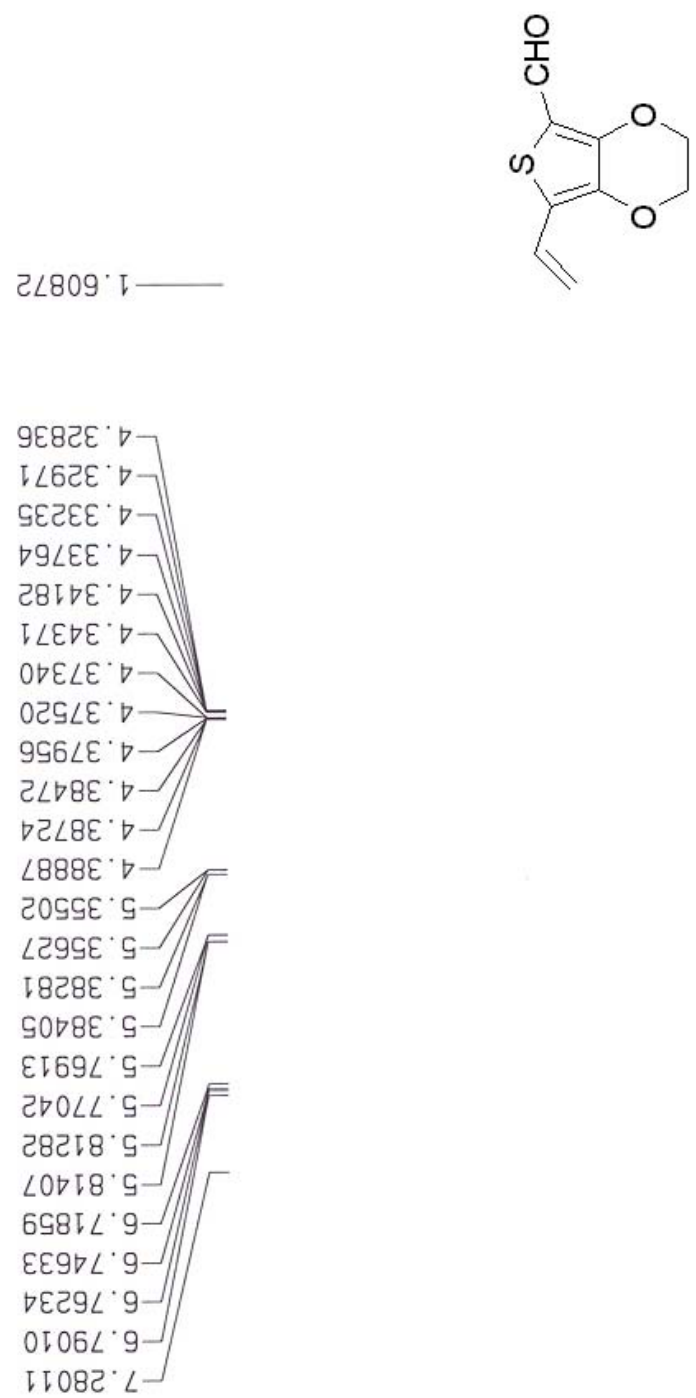

$\nabla 9906^{\circ} 6$

wdd

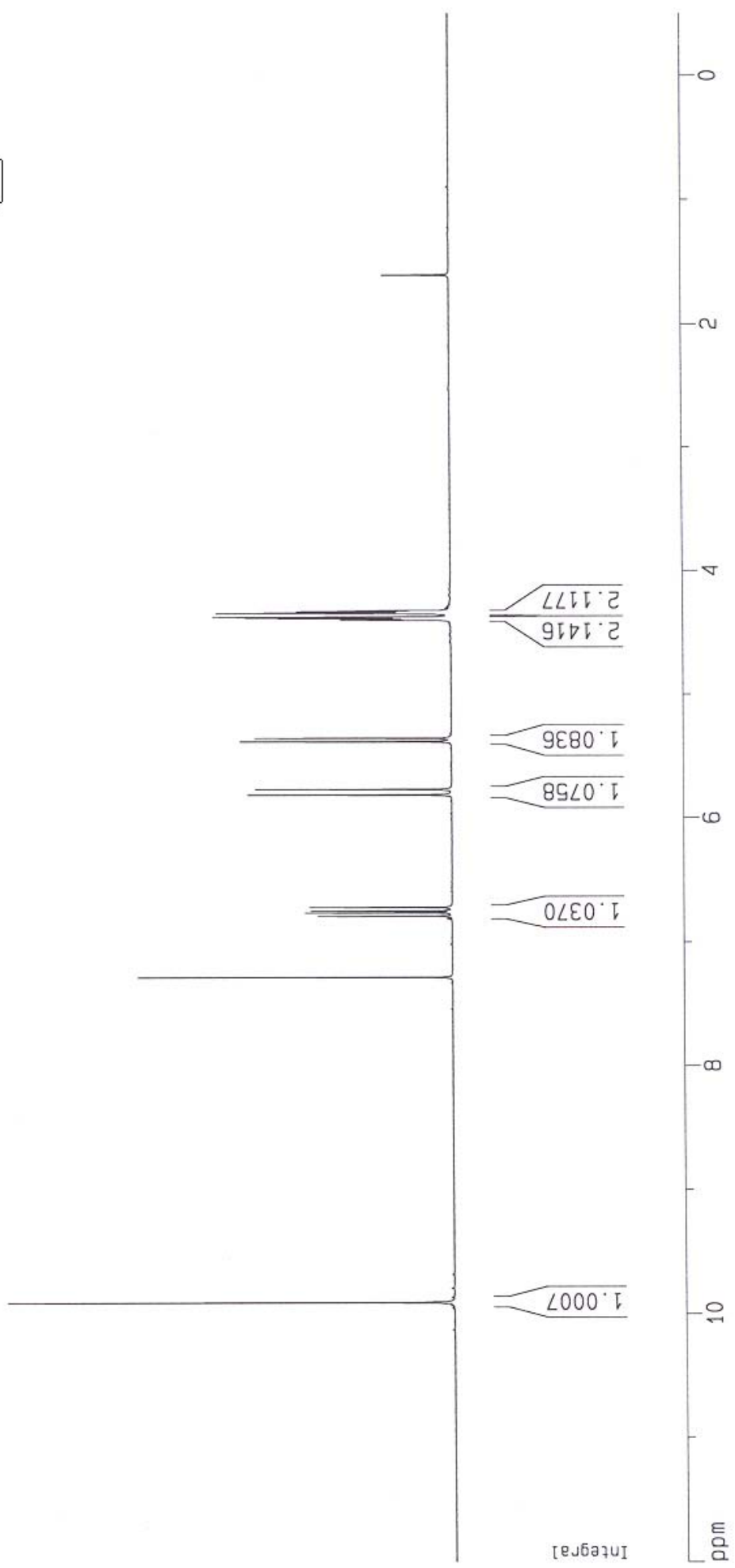

Figure S62: ${ }^{1} \mathrm{H}$ NMR of 19 


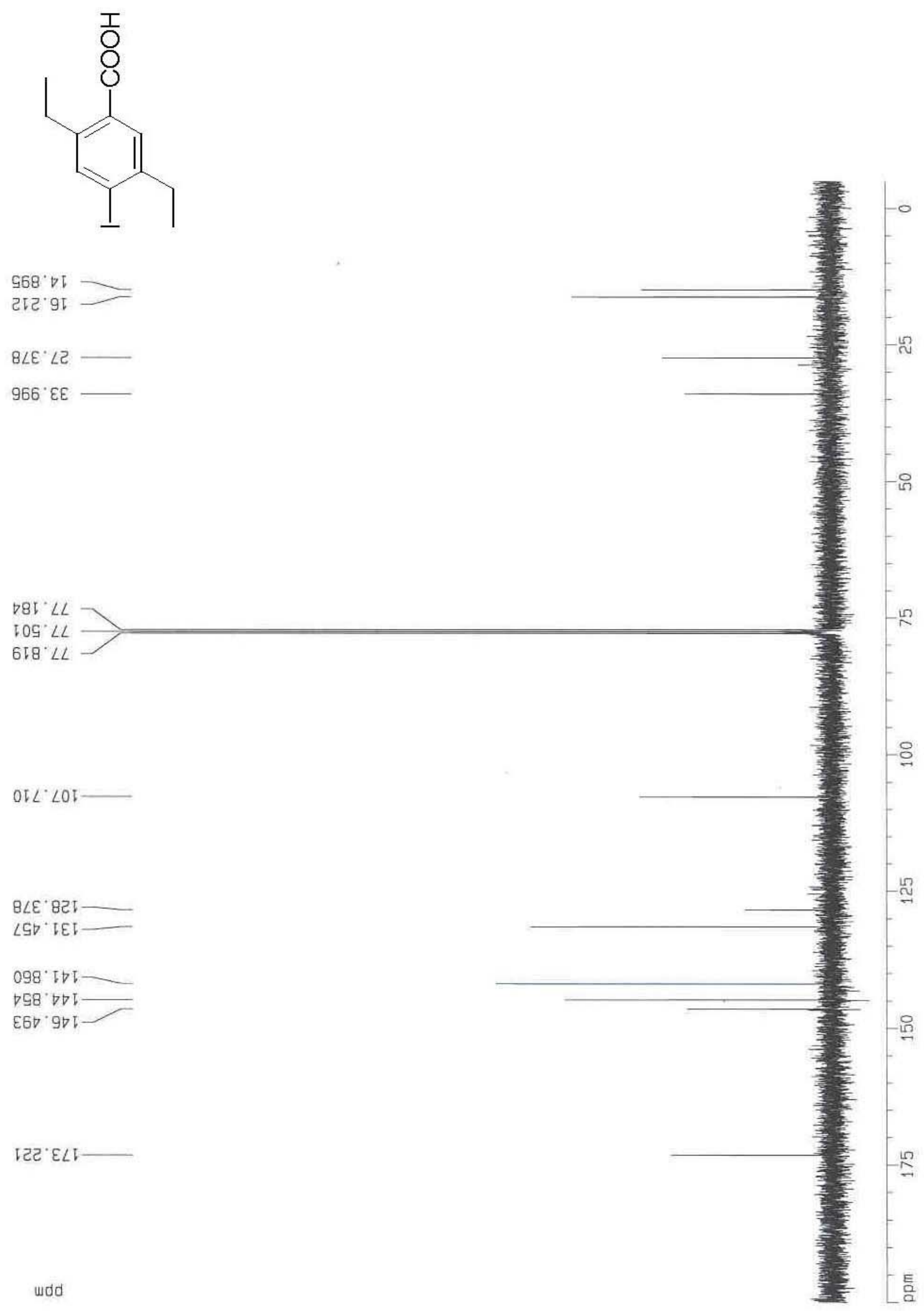

Figure S63: ${ }^{13} \mathrm{C}$ NMR of 22 


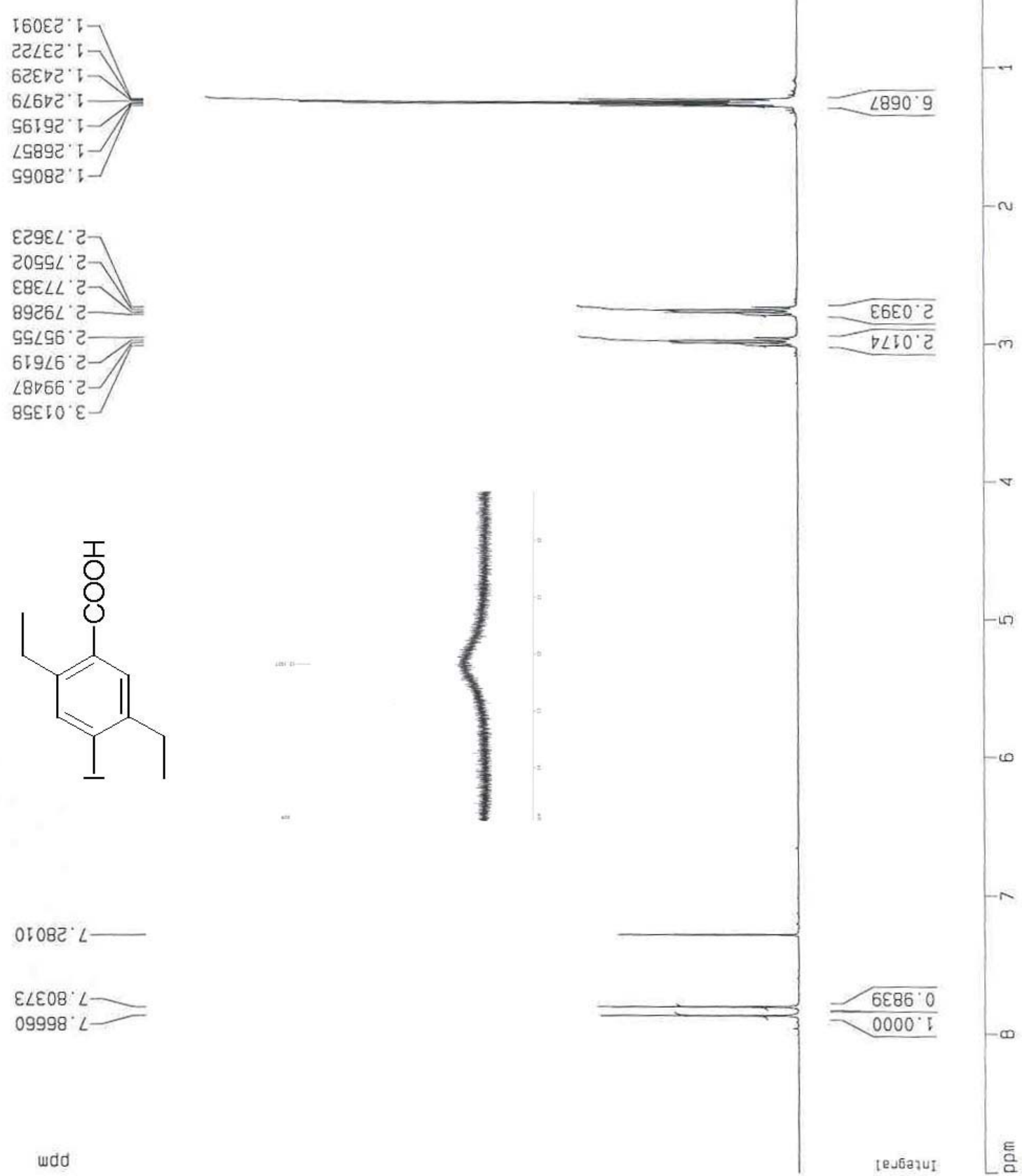

Figure S64: ${ }^{1} \mathrm{H}$ NMR of 22 
$\nabla 80^{\circ} 95$

OSE 9 โ

$\nabla 99^{\circ} \nabla 2$

โ८6 ' $\varepsilon \varepsilon$

เレ॰.29

$981^{\circ} \angle L$

$209^{\circ} \angle L$

$028^{\circ} L L$

$906^{\circ} 66$

ह०Г'8टा-

$\subseteq E L \cdot 8 E \downarrow$ $\angle O E^{\circ} 6 E[\longrightarrow$

SI9. IDI -

$\nabla \nabla \varepsilon^{\circ} \nabla \nabla L$

wdd

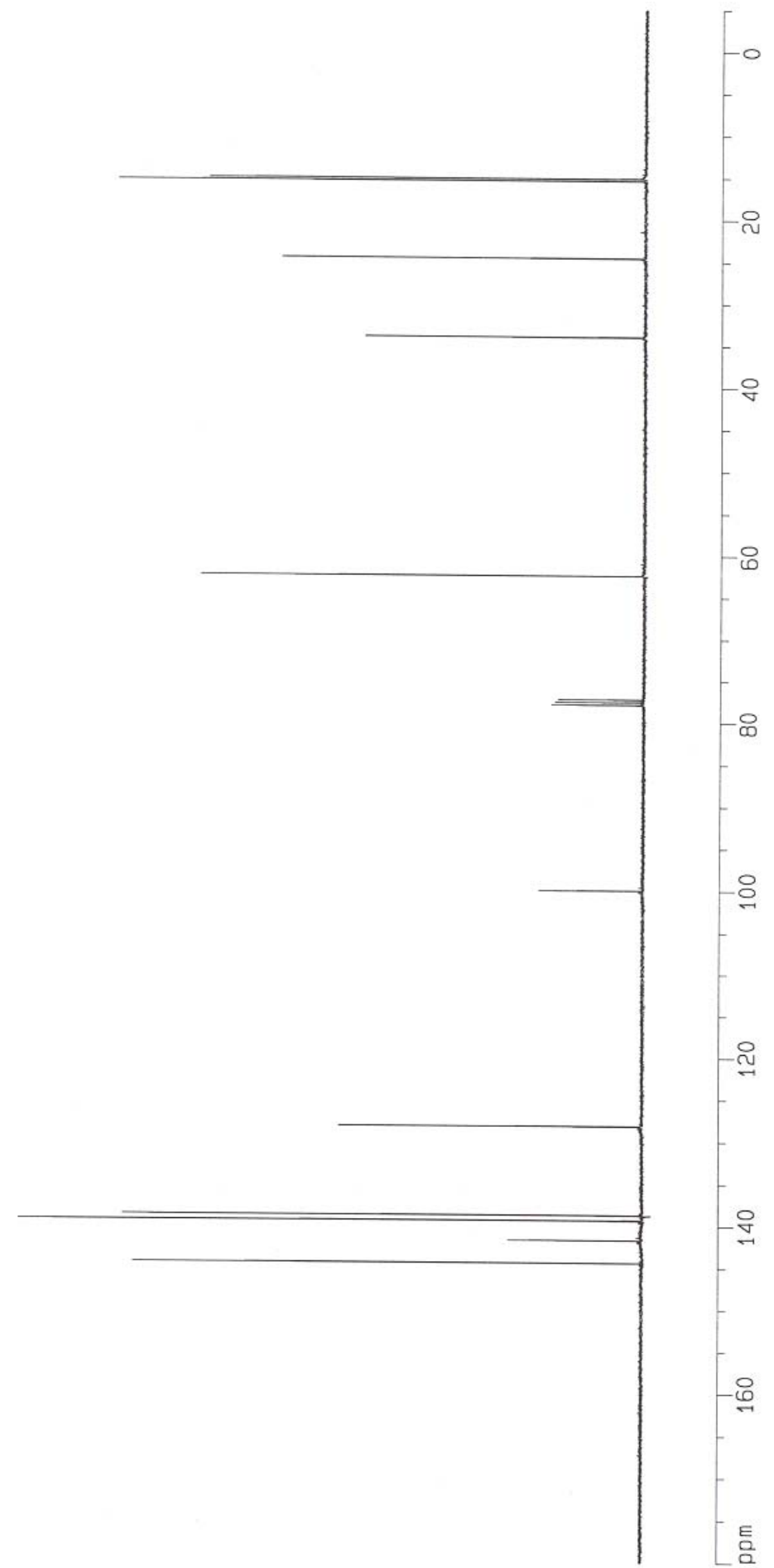

Figure S65: ${ }^{13} \mathrm{C}$ NMR of $\mathbf{2 3}$ 


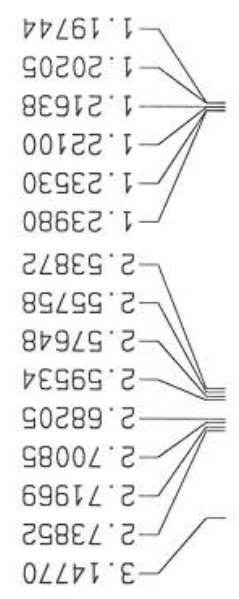

99टद与 *

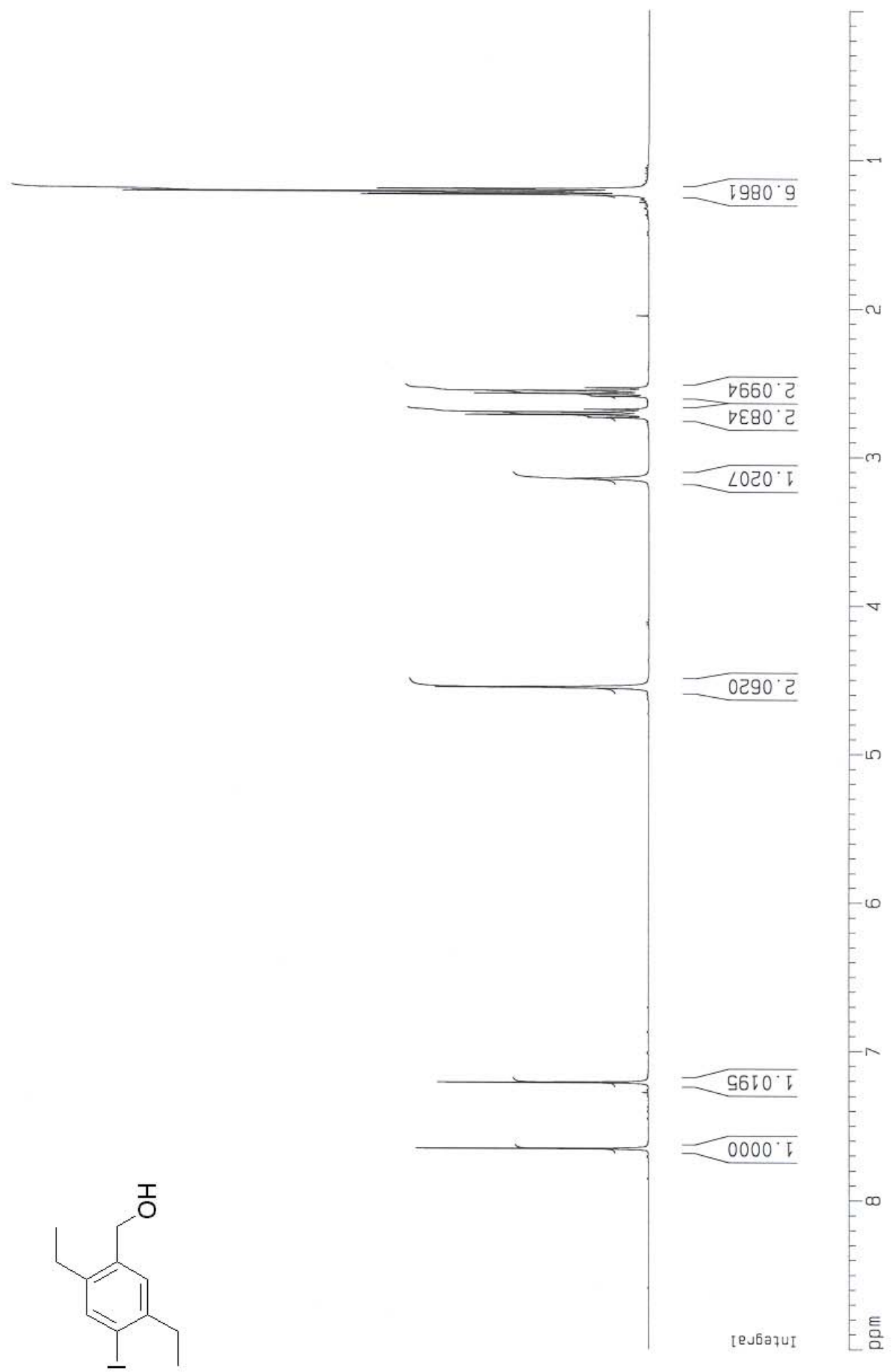

wdd

Figure S66: ${ }^{1} \mathrm{H}$ NMR of $\mathbf{2 3}$ 


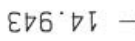

$9 \angle \digamma G T$

$\varepsilon 99^{\circ} \nabla ट$

I9t' IE

เ98. $\varepsilon \varepsilon$

I81' $\angle L$

$66 \nabla^{\circ} \angle L$

$918^{\circ} L L$

208. ก

$\angle 己 \nabla^{\circ} O E \downarrow-$

E68. GE ᄂ -

9เ6. $6 ย \downarrow$

$\angle O S^{\circ} 2 \nabla V$

$8 \nabla L^{\circ} \nabla \nabla L$

wdd

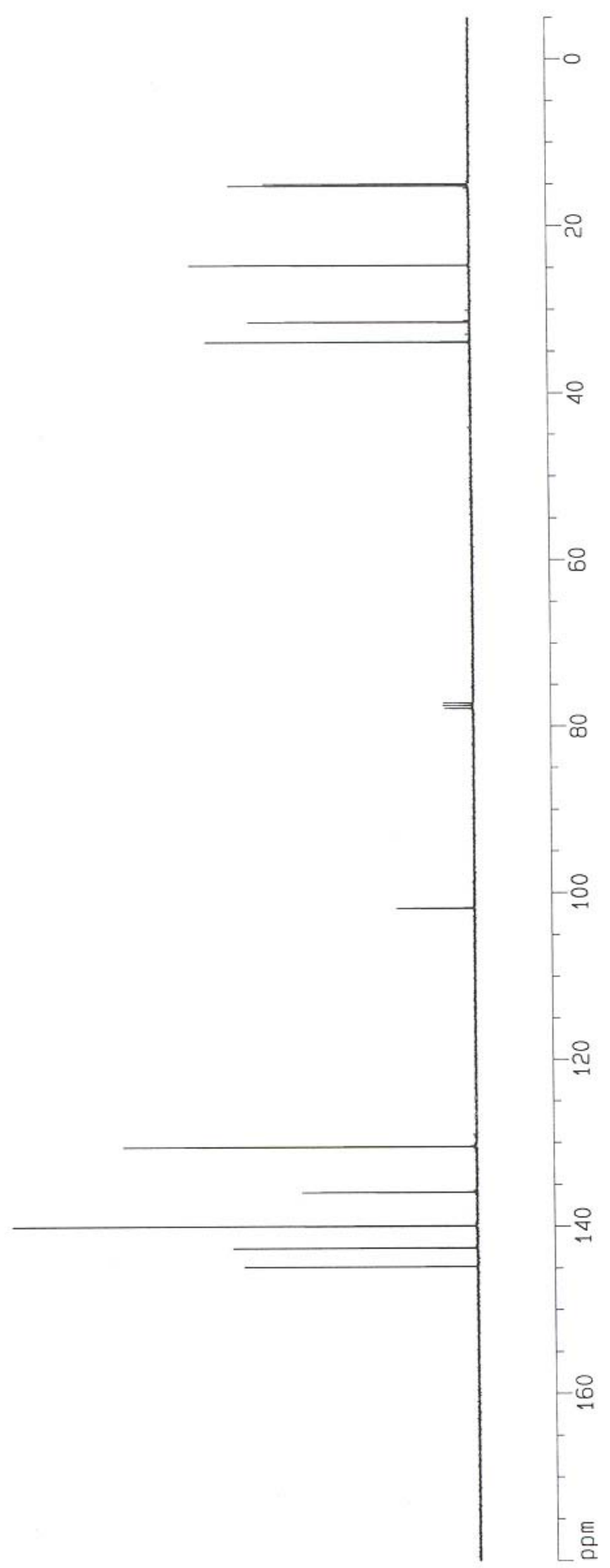

Figure S67: ${ }^{13} \mathrm{C}$ NMR of $\mathbf{2 4}$ 


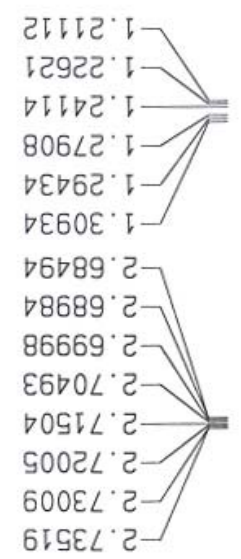

与668 $\nabla^{\circ}$

IDV $\angle I \cdot L$

I $\angle \angle C^{\circ} \angle$

ᄂ8189 $\mathrm{L}$

wdd

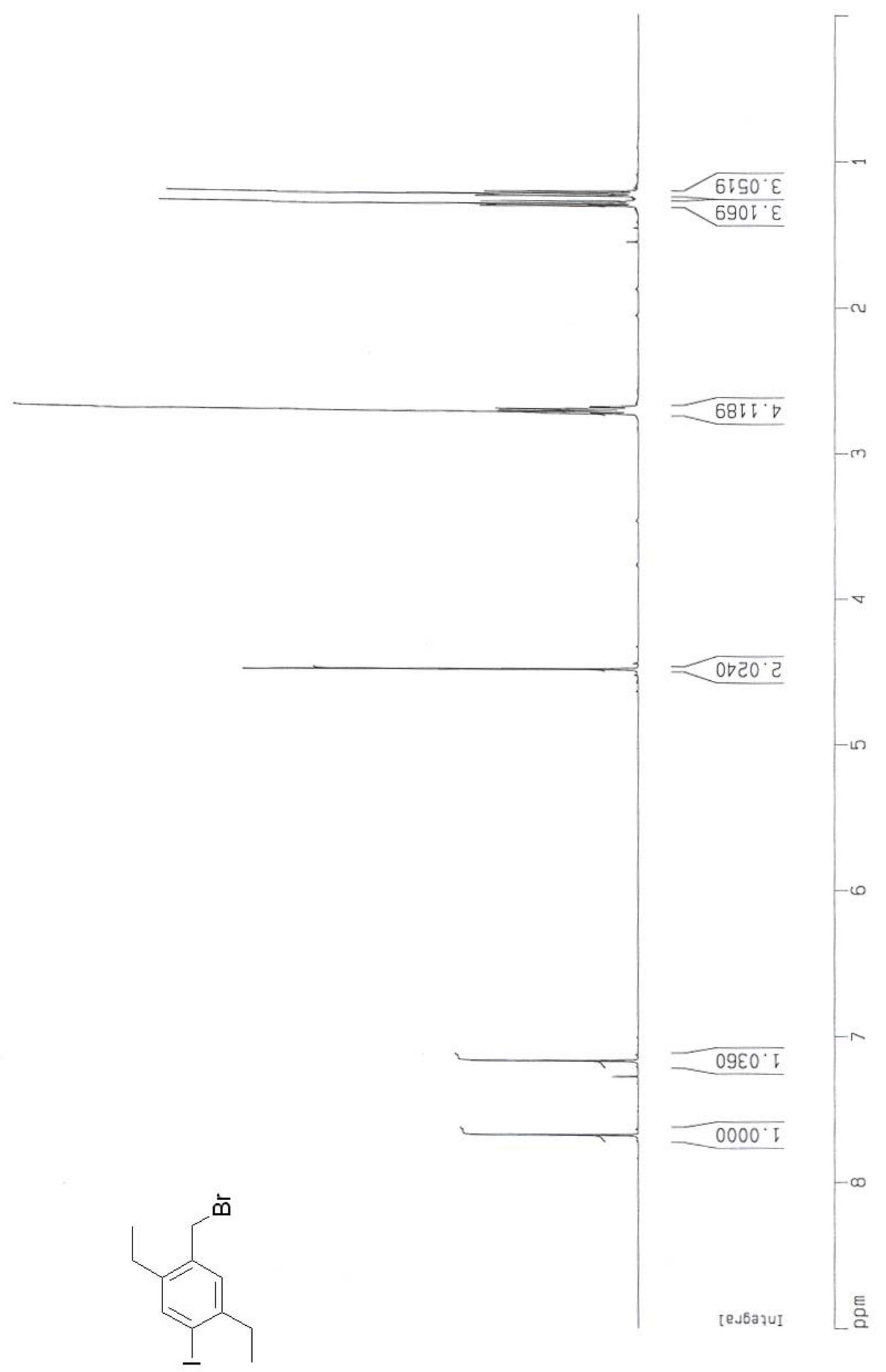

Figure S68: ${ }^{1} \mathrm{H}$ NMR of $\mathbf{2 4}$ 

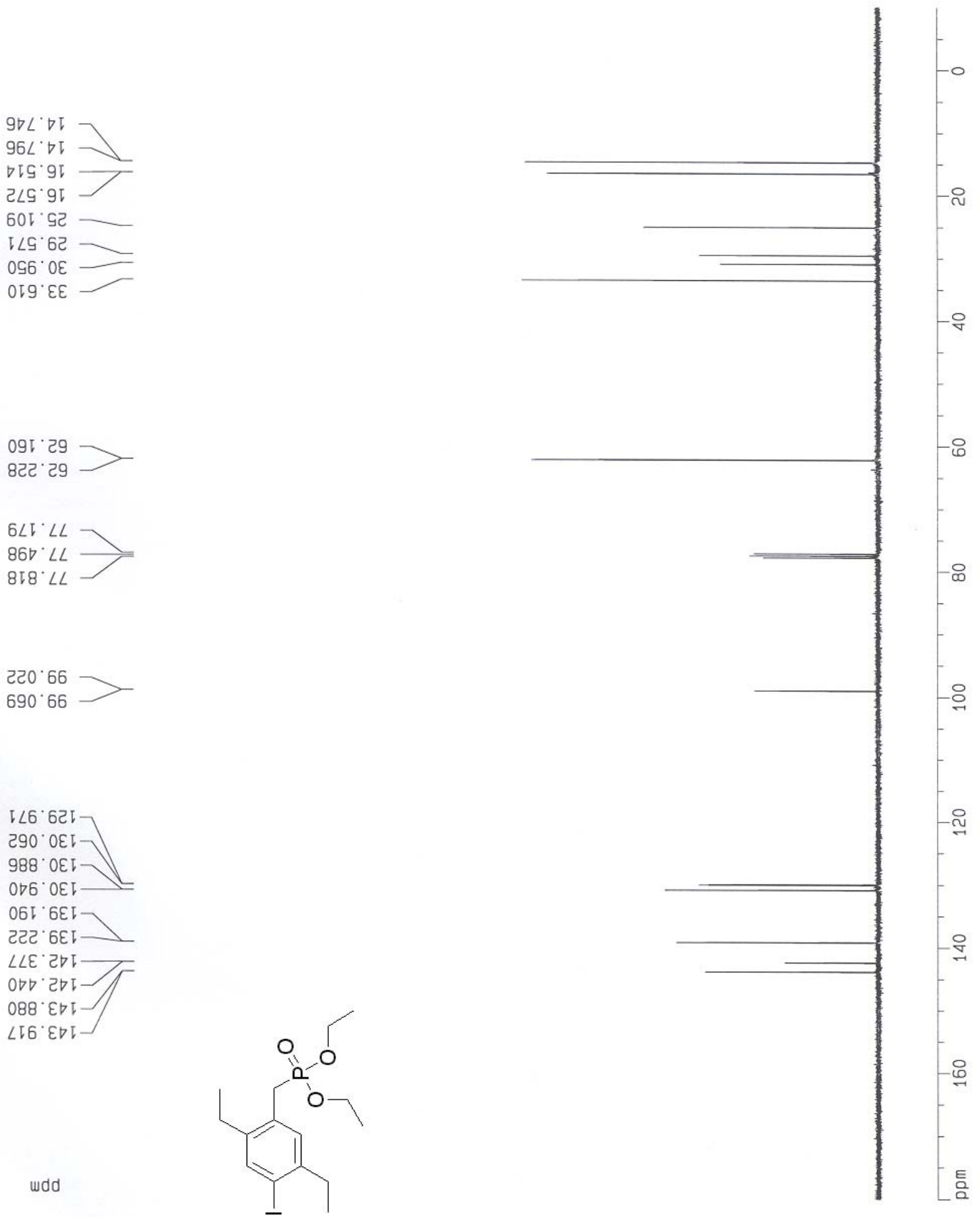

Figure S69: ${ }^{13} \mathrm{C}$ NMR of 25 

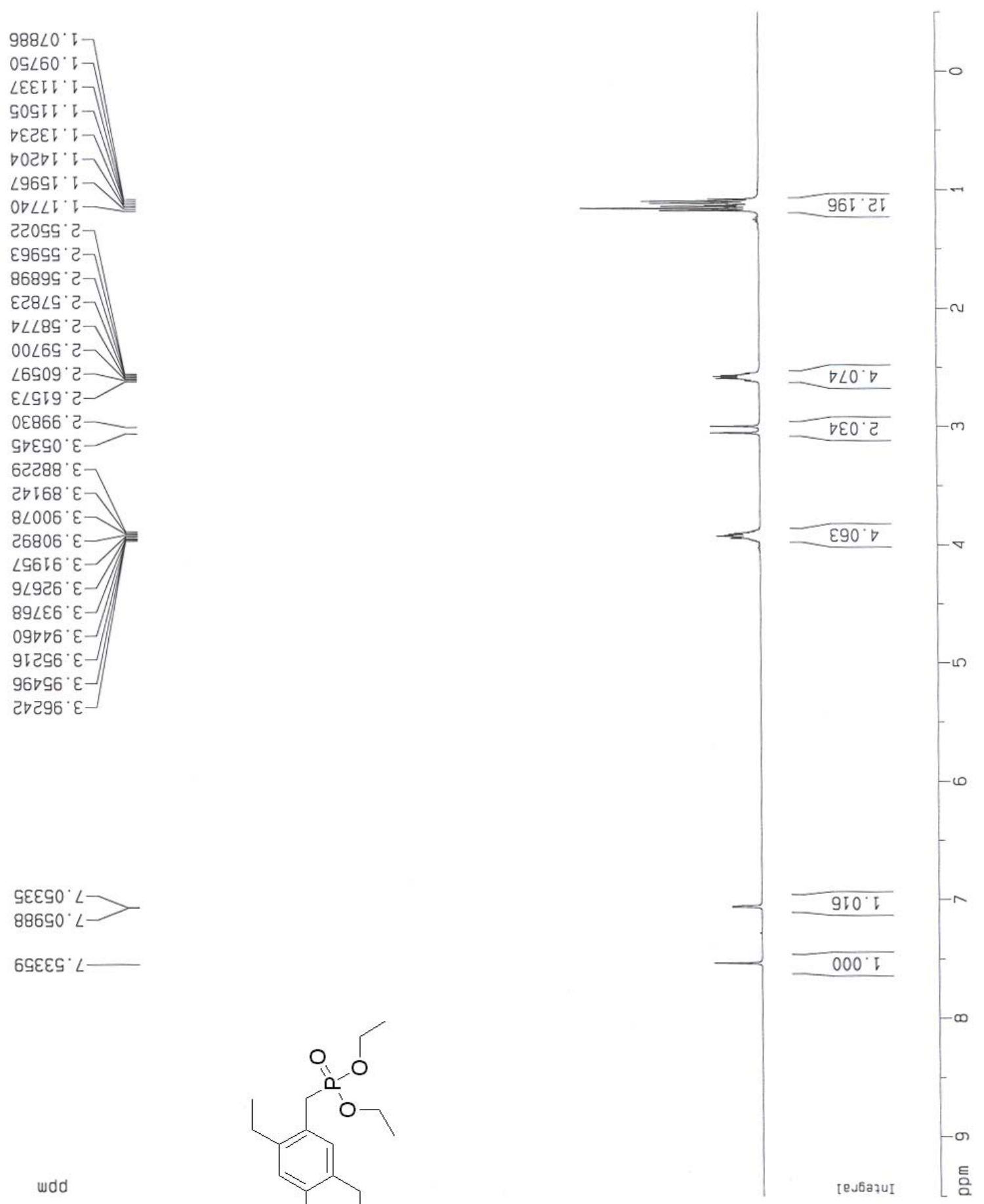

wdd

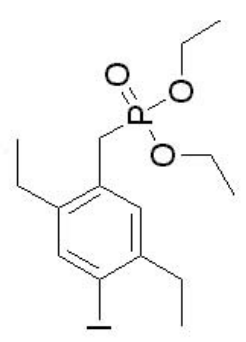

Figure S70: ${ }^{1} \mathrm{H}$ NMR of $\mathbf{2 5}$ 


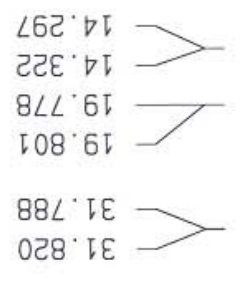

$9 \angle 2 \cdot 29$

$\angle 00.69$

$\varepsilon \angle \varepsilon^{\circ} O L$ $660^{\circ} \angle L$

टS $L^{\circ} \angle L$

$6 \nabla \nabla^{\circ} 98$

GOE' $\varepsilon โ$

129 टटઈ-

$898^{\circ}$ OE

$O 2 L$ ISI

6ट૬' टפा-

wdd

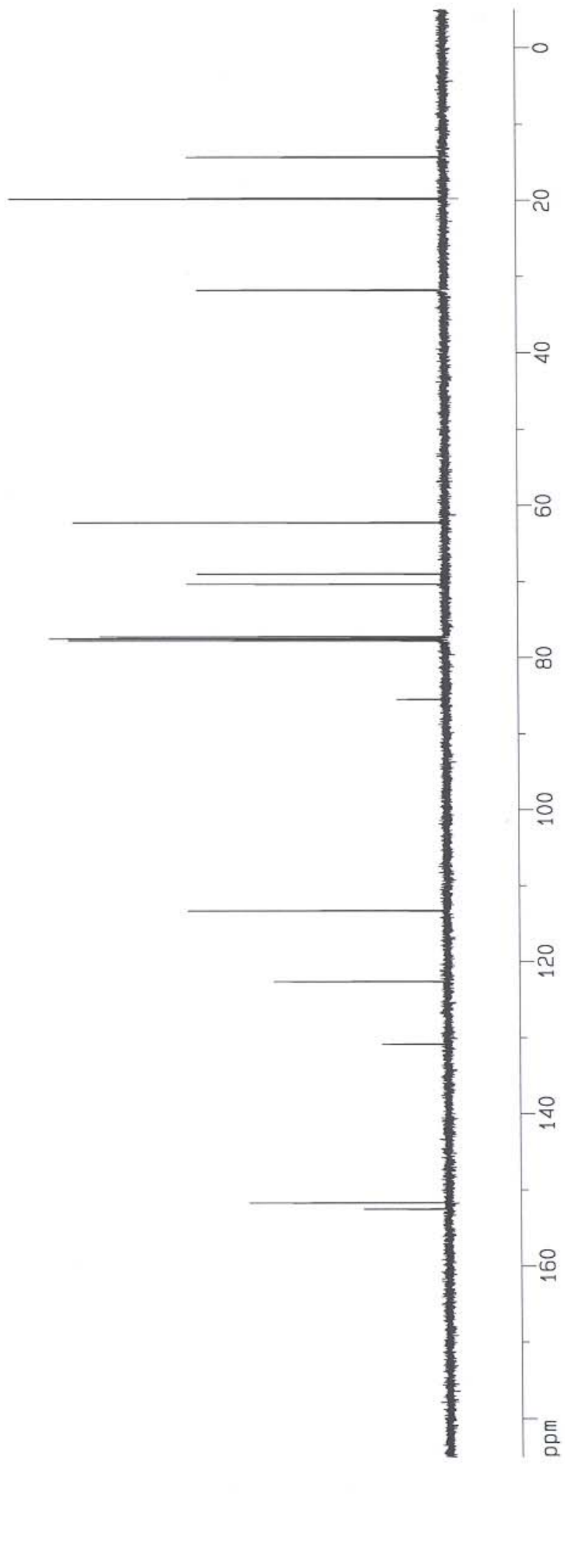

Figure S71: ${ }^{13} \mathrm{C}$ NMR of 26 

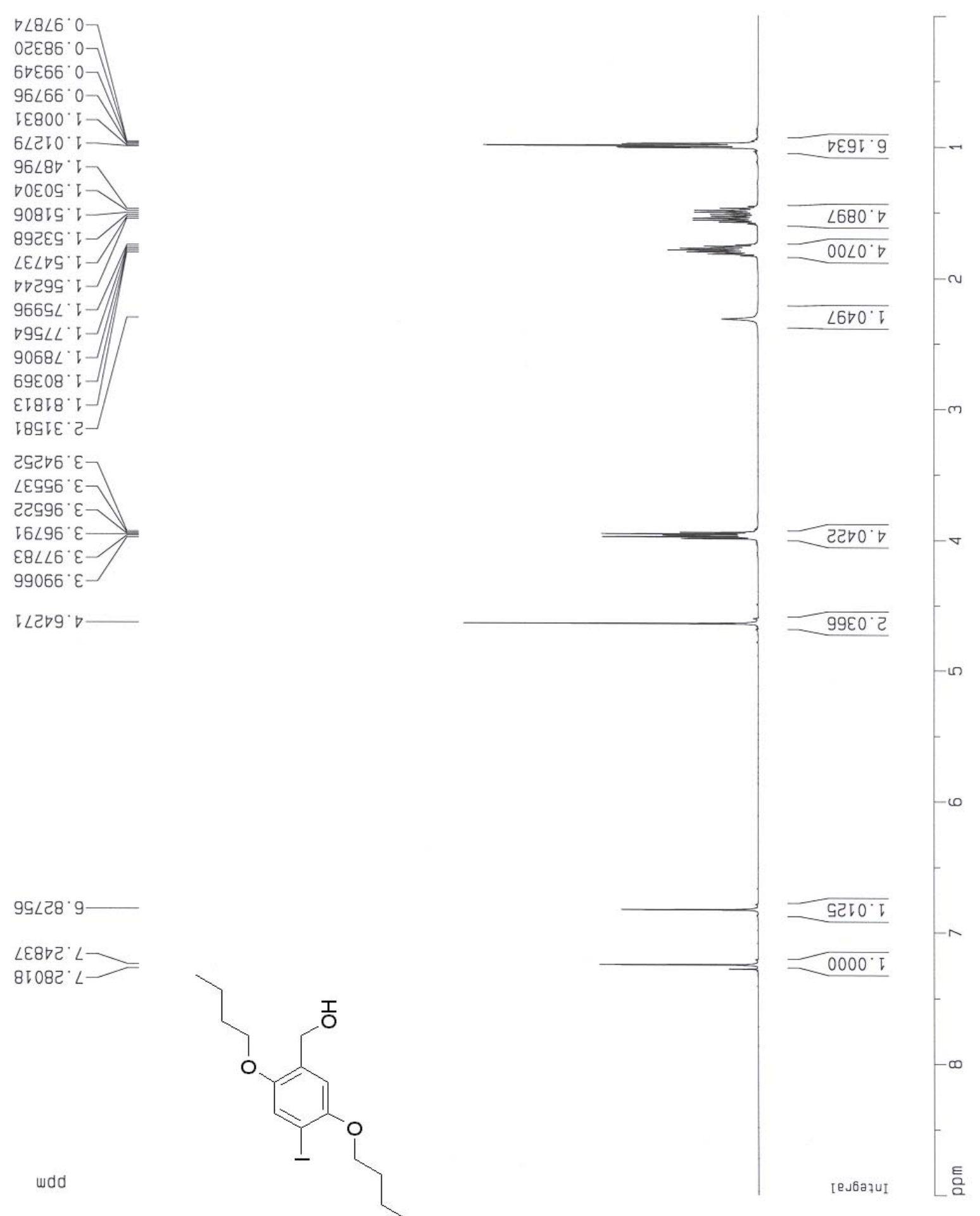

$99 \angle 28 \cdot 9$

$\angle \varepsilon 8 \nabla C^{\circ} \angle \longrightarrow$
$8108 C^{\circ} \angle \longrightarrow$

wdd

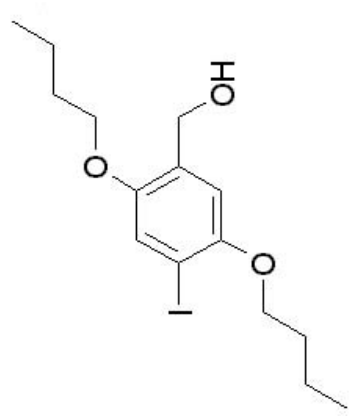

Figure S72: ${ }^{1} \mathrm{H}$ NMR of $\mathbf{2 6}$ 

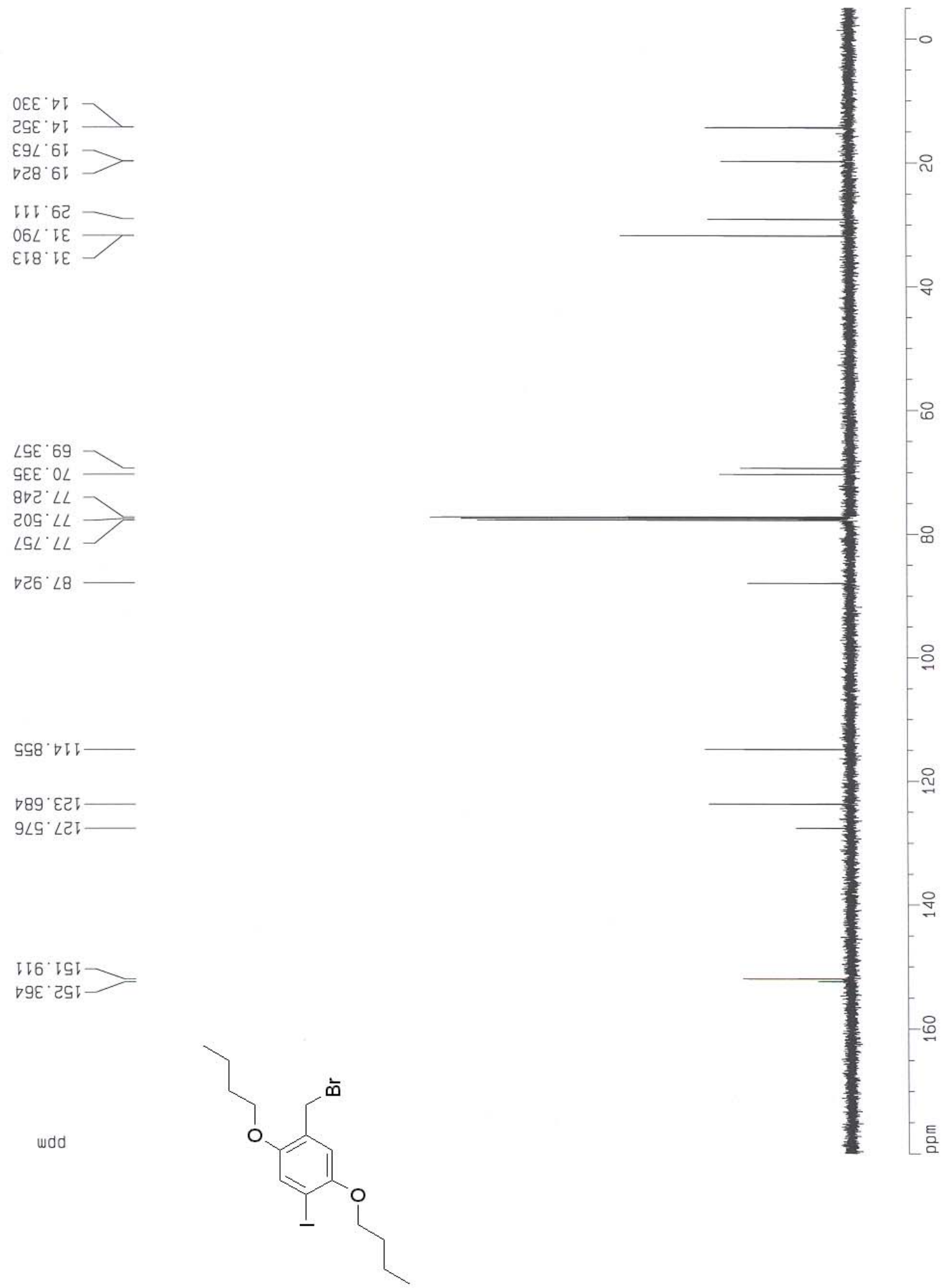

$\nabla 26 \cdot \angle 8$

ธุ8 จレ——

$\nabla 89^{\circ}$ Е己Г—

$9 \angle 9^{\circ} \angle 2 \downarrow$

II6 ISI-

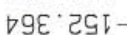

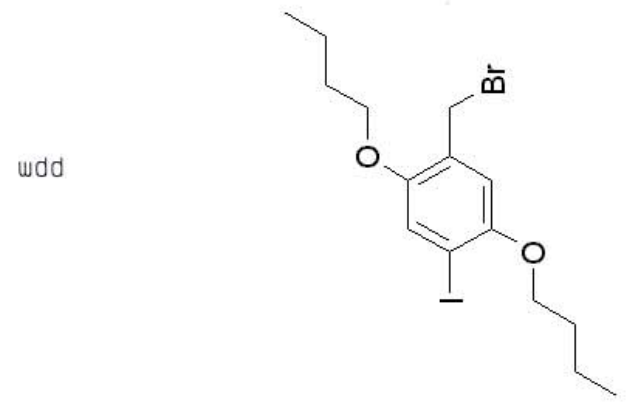

Figure S73: ${ }^{13} \mathrm{C}$ NMR of 27 

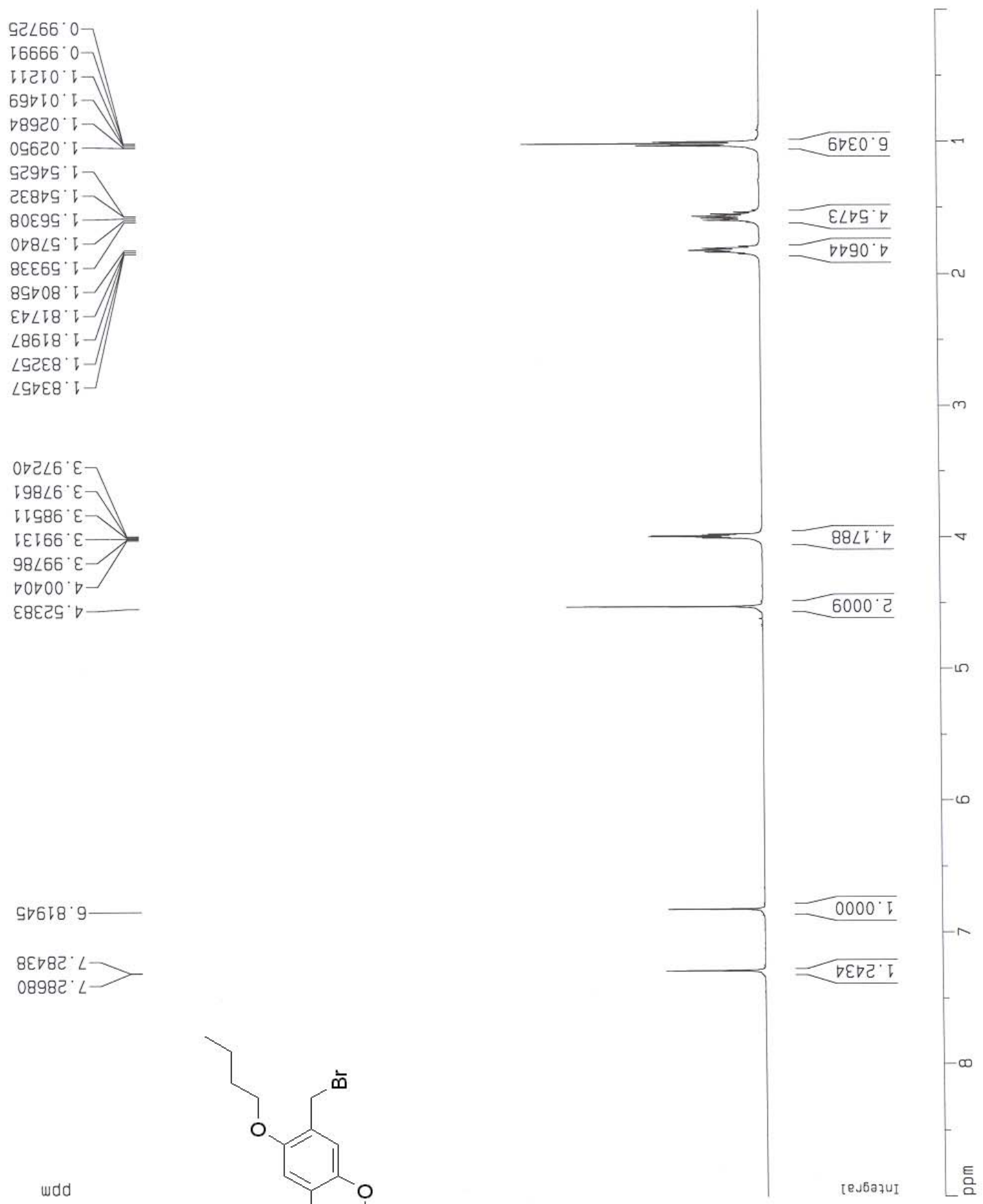

5จ6เ8.9

৪E॰8ट $\angle$ $08982: L$

wdd

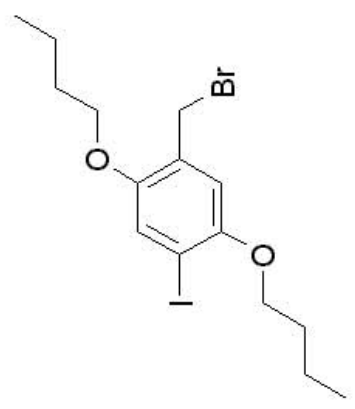

Figure S74: ${ }^{1} \mathrm{H}$ NMR of 27 

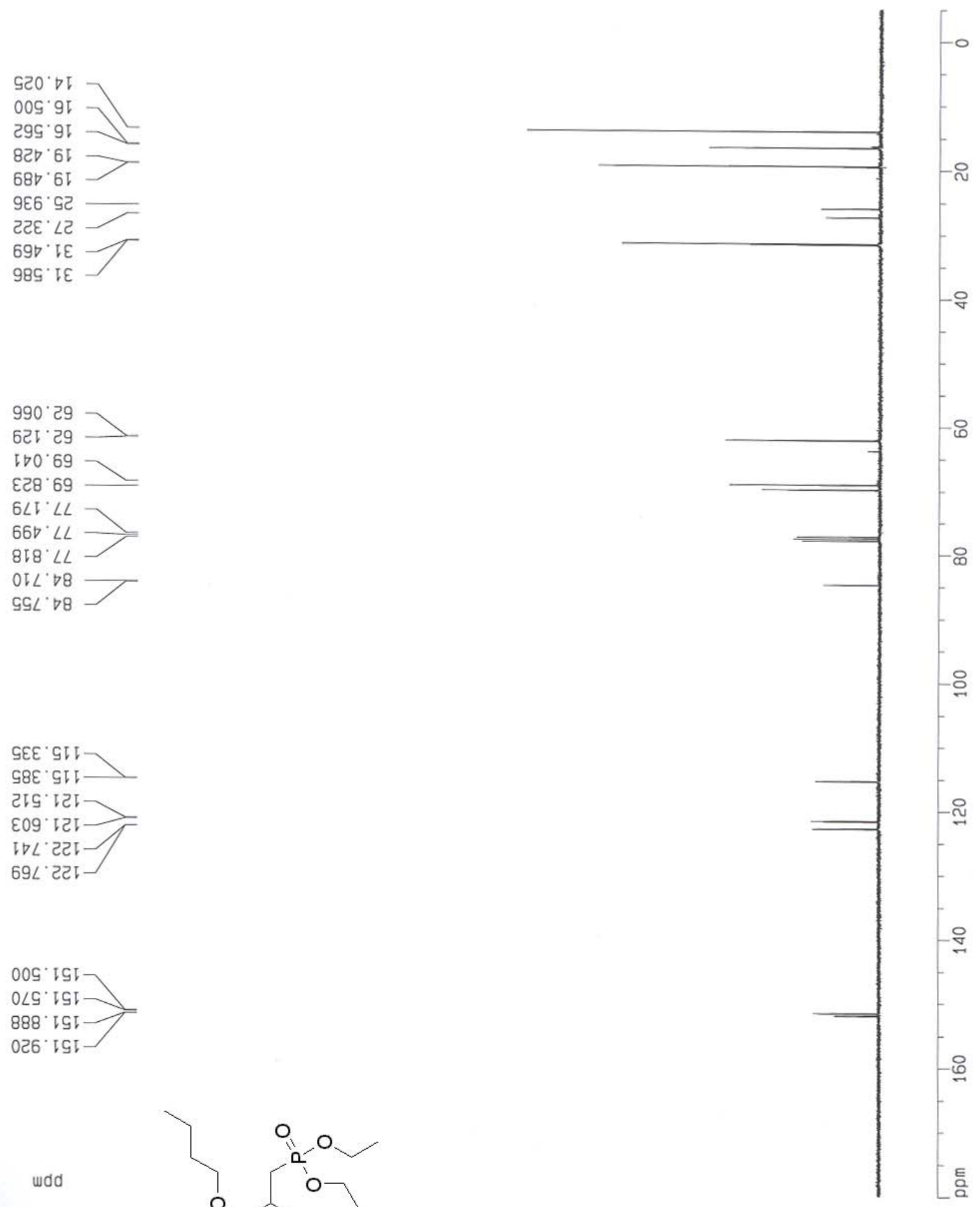

wdd

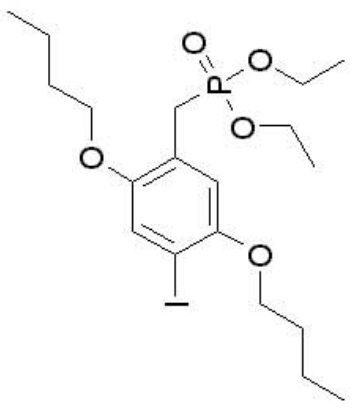

Figure S75: ${ }^{13} \mathrm{C}$ NMR of $\mathbf{2 8}$ 

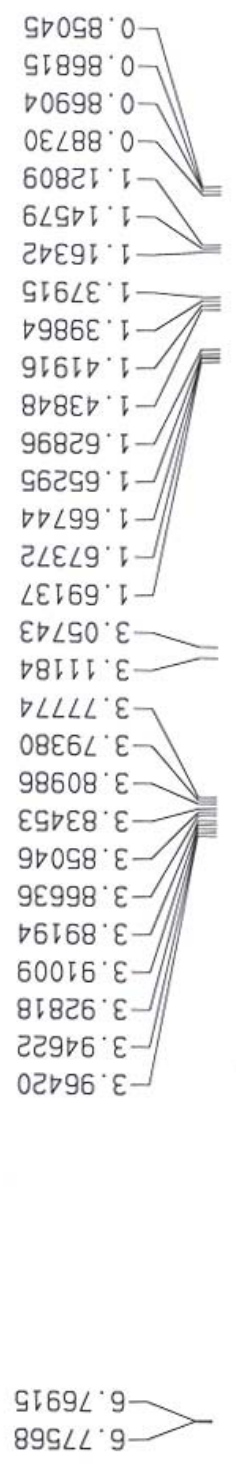

与Eटレ

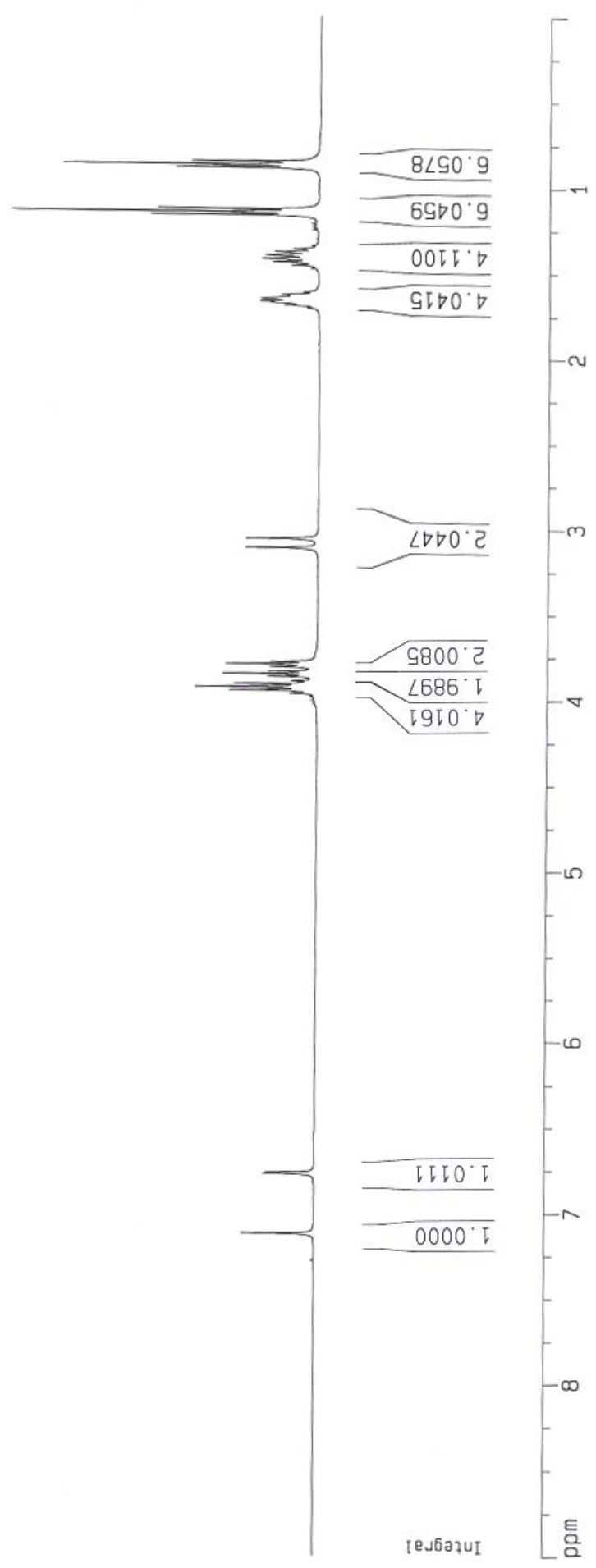

Figure S76: ${ }^{1} \mathrm{H}$ NMR of $\mathbf{2 8}$ 


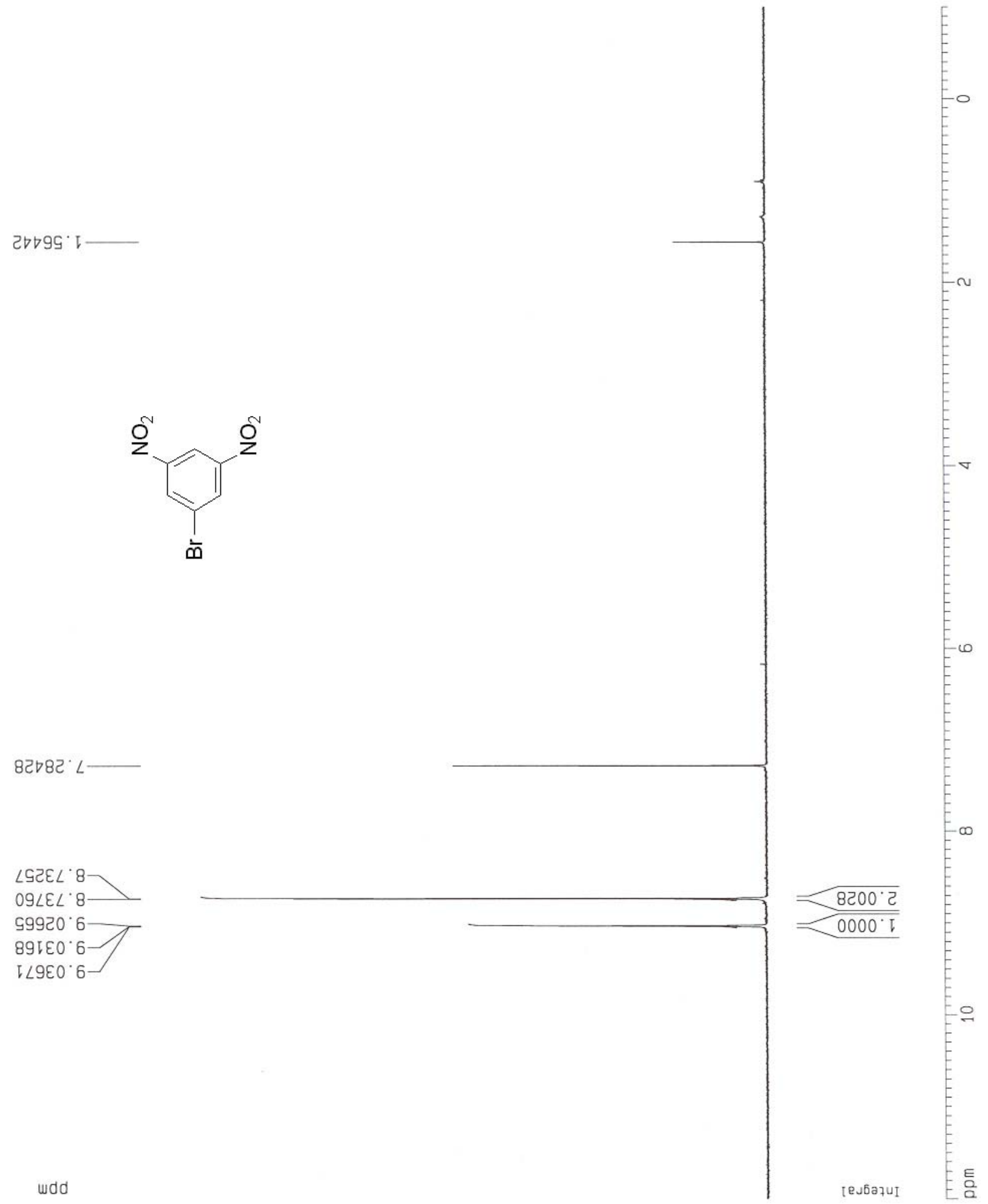

Figure S77: ${ }^{1} \mathrm{H}$ NMR of 32 


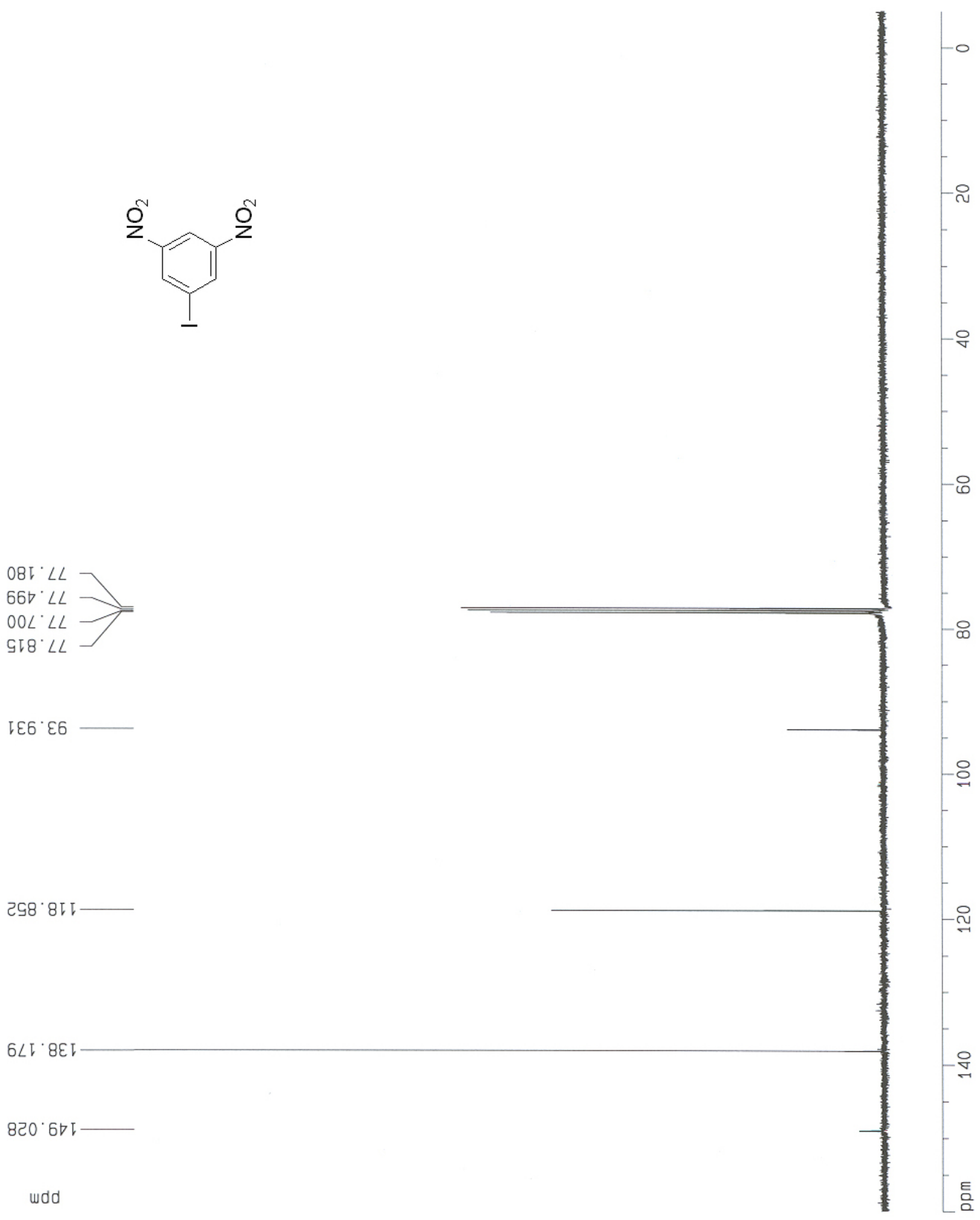

Figure S78: ${ }^{13} \mathrm{C}$ NMR of $\mathbf{3 3}$ 


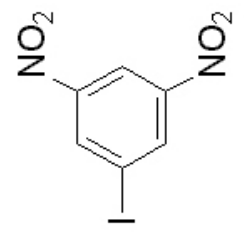

$866 \angle 2 \cdot L$

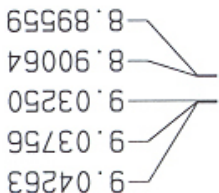

wdd

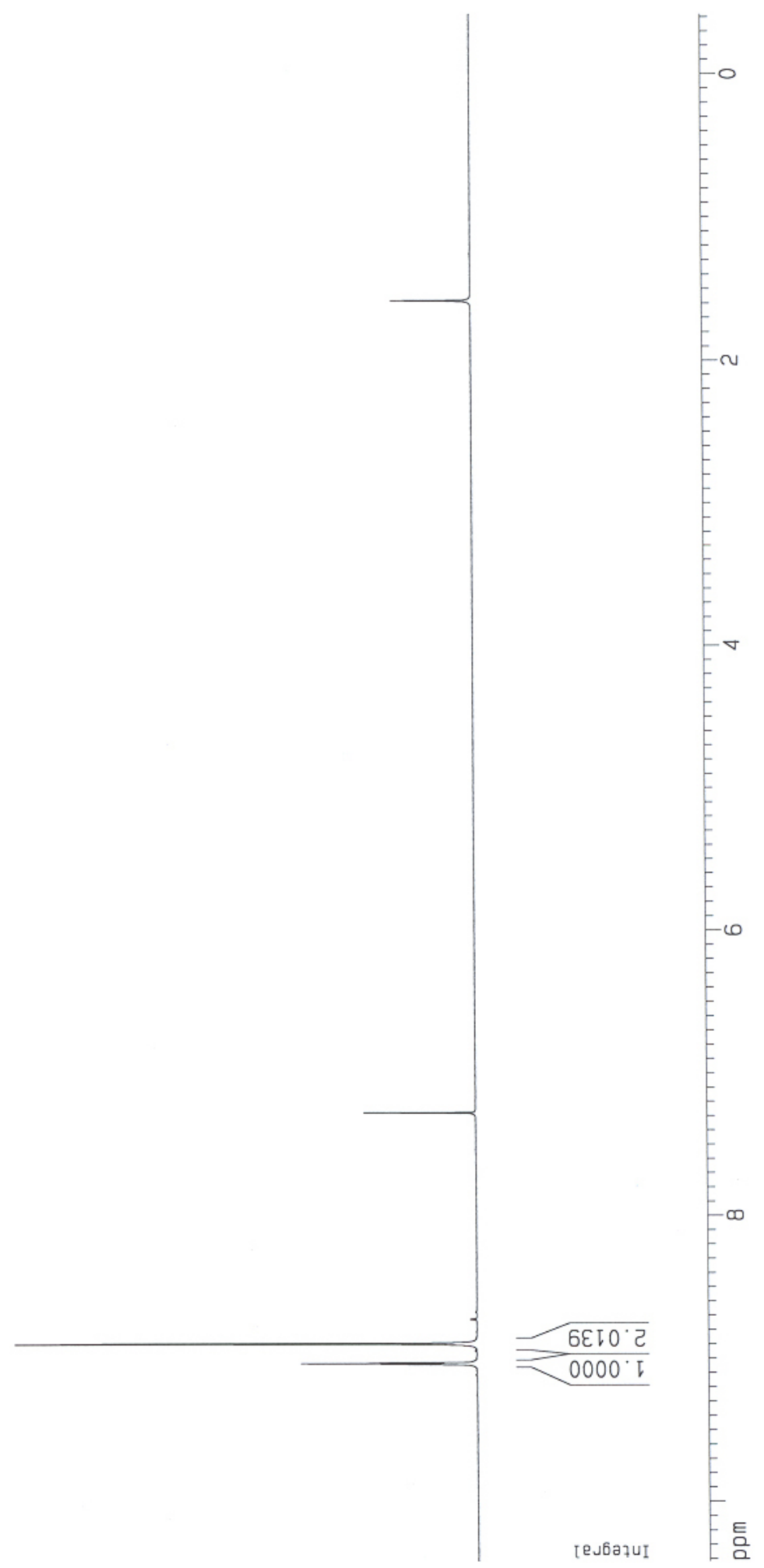

Figure S79: ${ }^{1} \mathrm{H}$ NMR of $\mathbf{3 3}$ 


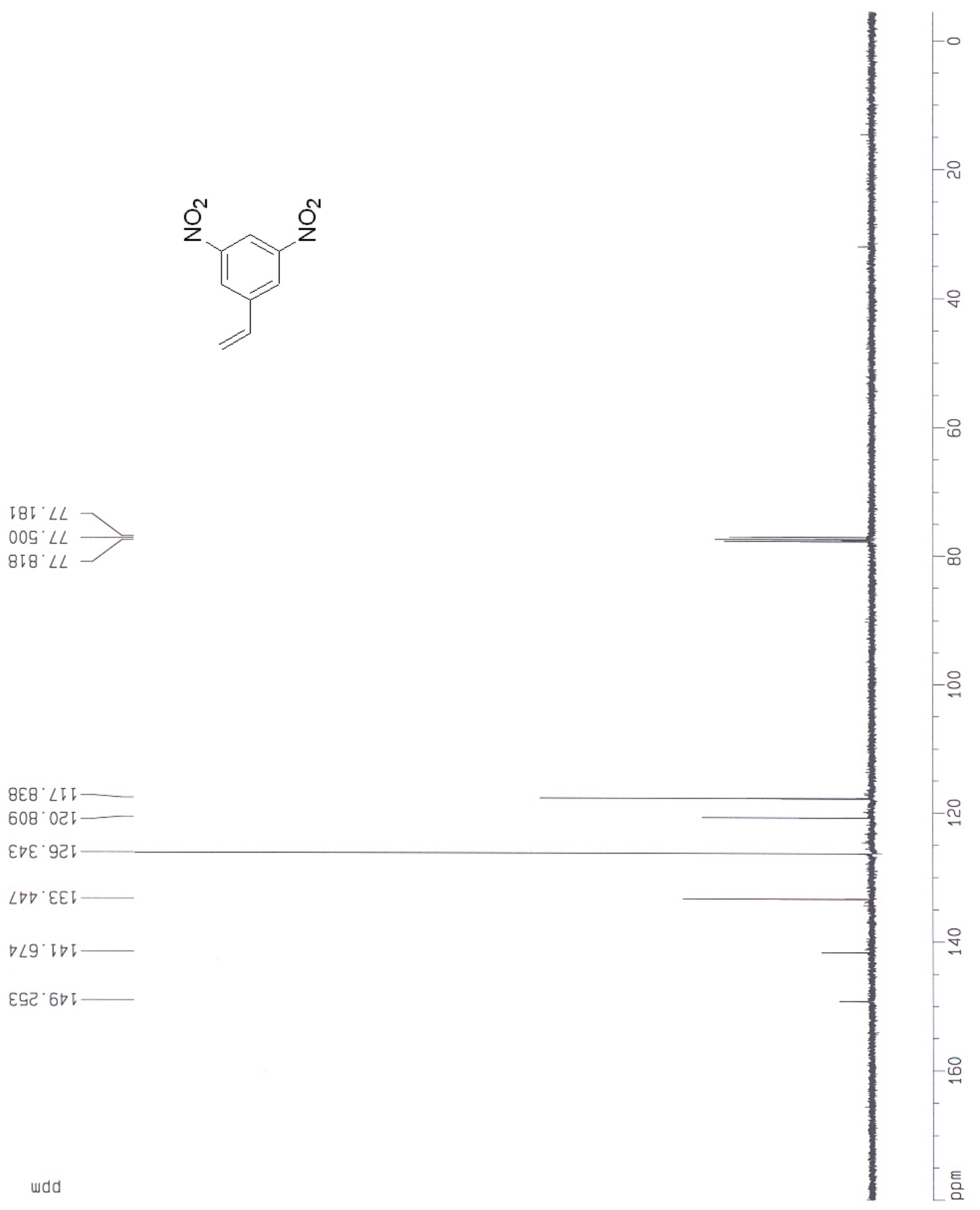

Figure S80: ${ }^{13} \mathrm{C}$ NMR of 34 

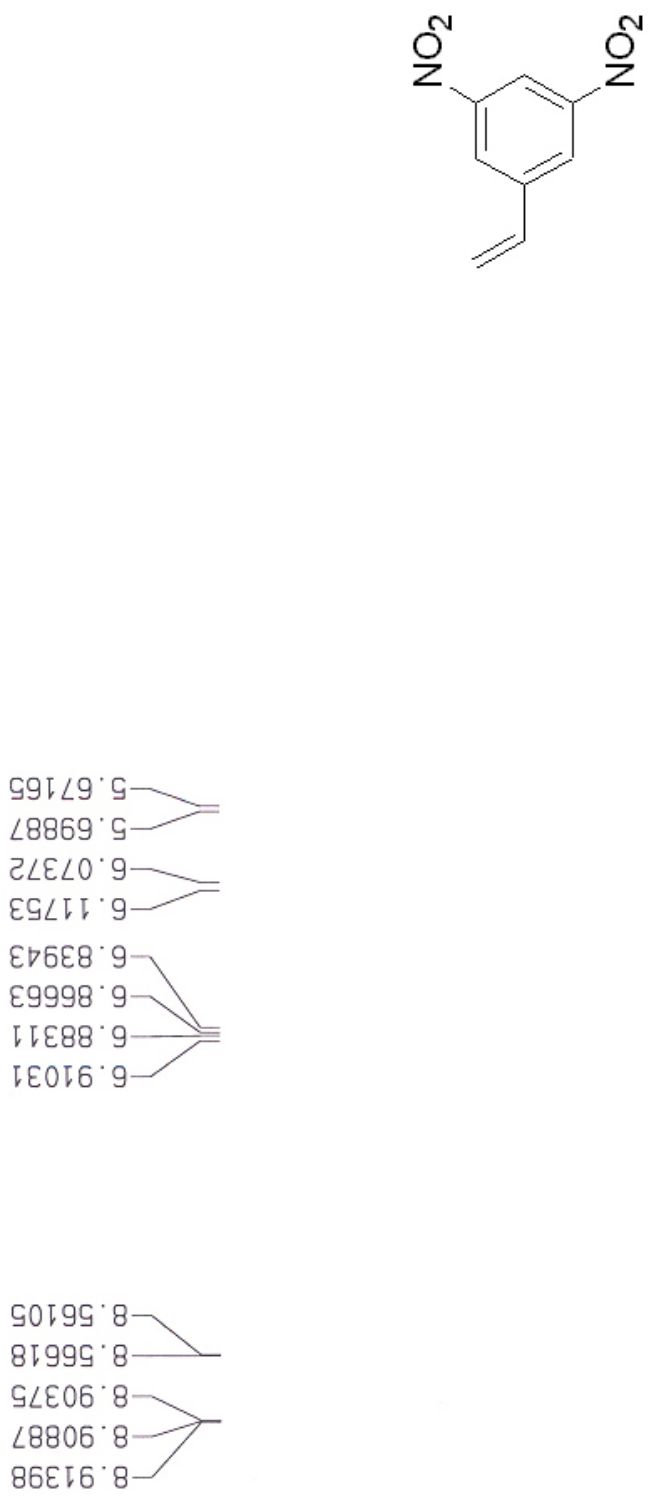

wdd

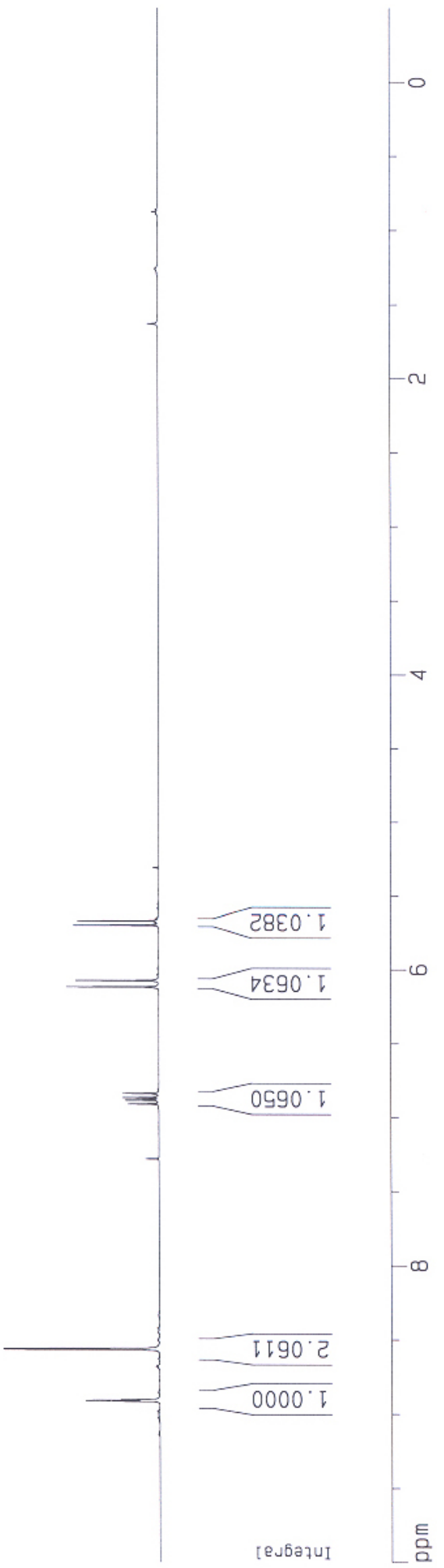

Figure S81: ${ }^{1} \mathrm{H}$ NMR of $\mathbf{3 4}$ 

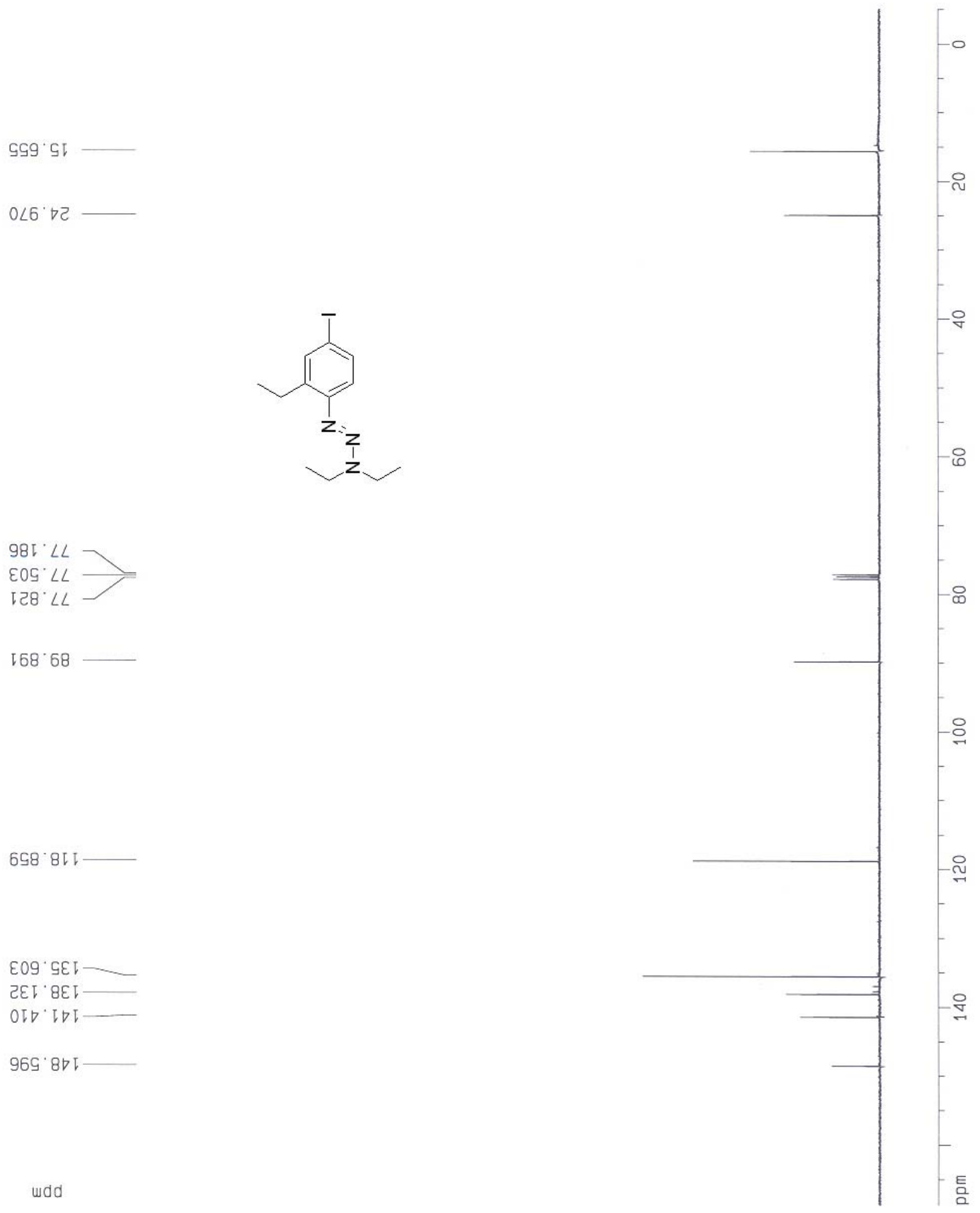

$98 I^{\prime} \angle L$

EOS $\angle L$

Iट8 $\angle L$

โ68. 68

$698^{\circ} 815$

E09 $9 \varepsilon \downarrow \longrightarrow$

टहा ' $8 E \downarrow$

OLV IDI -

$969^{\circ} 8 \nabla \tau^{2}$

wdd

$\circ$

요

우

(1)

8

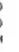

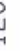

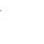

的

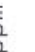

Figure S82: ${ }^{13} \mathrm{C}$ NMR of $\mathbf{3 5 a}$ 

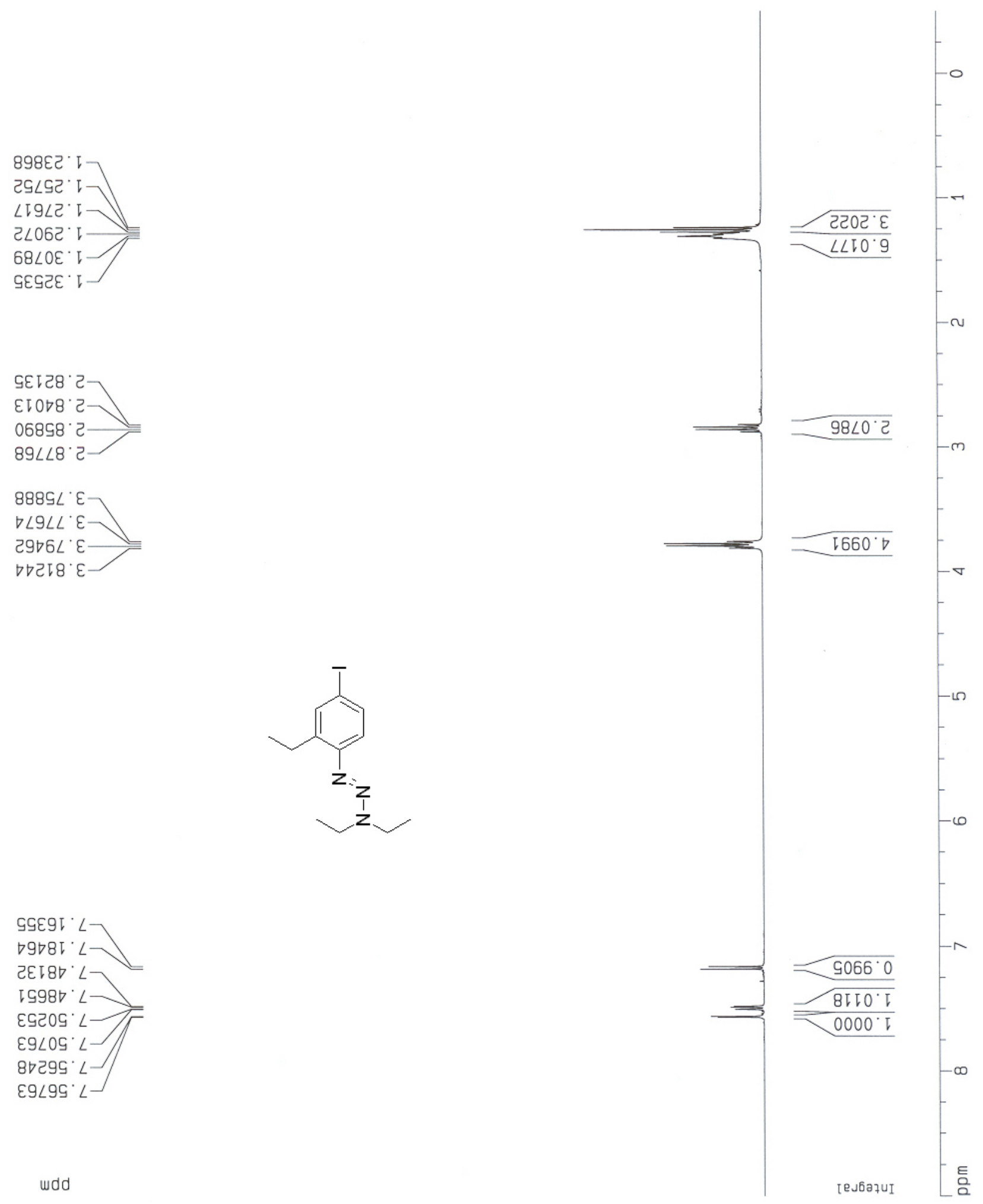

Figure S83: ${ }^{1} \mathrm{H}$ NMR of $\mathbf{3 5 a}$ 


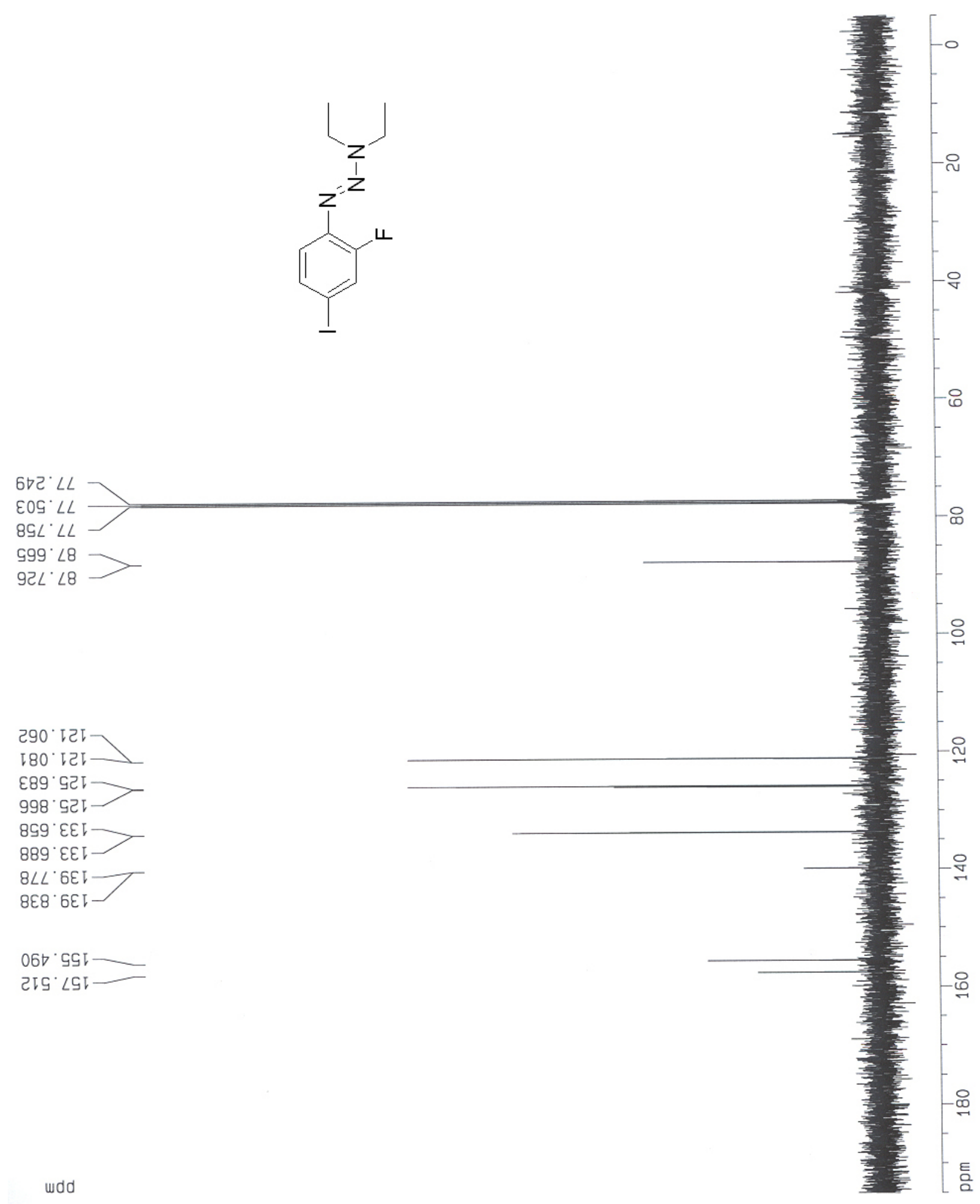

Figure S84: ${ }^{13} \mathrm{C}$ NMR of $\mathbf{3 5 c}$ 

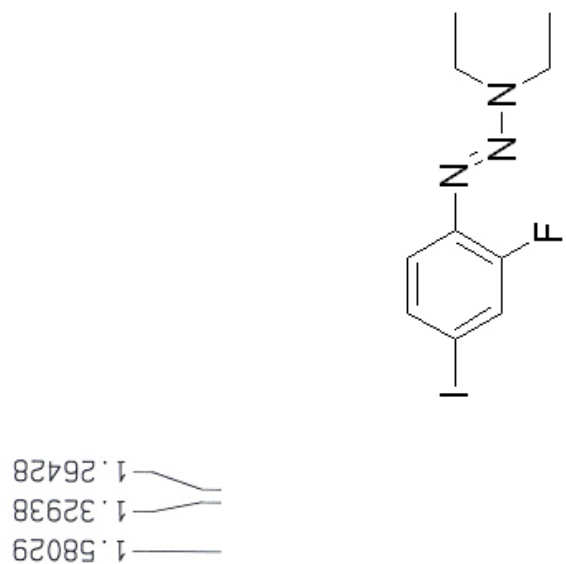

$\angle 68 \angle L^{\prime} \varepsilon$ OE६6 $\angle$ ' $\varepsilon$ $\varepsilon 9 \angle 08^{\circ} \varepsilon$ 86เट8 $\varepsilon$

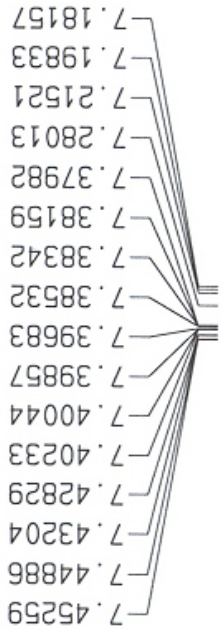

wdd

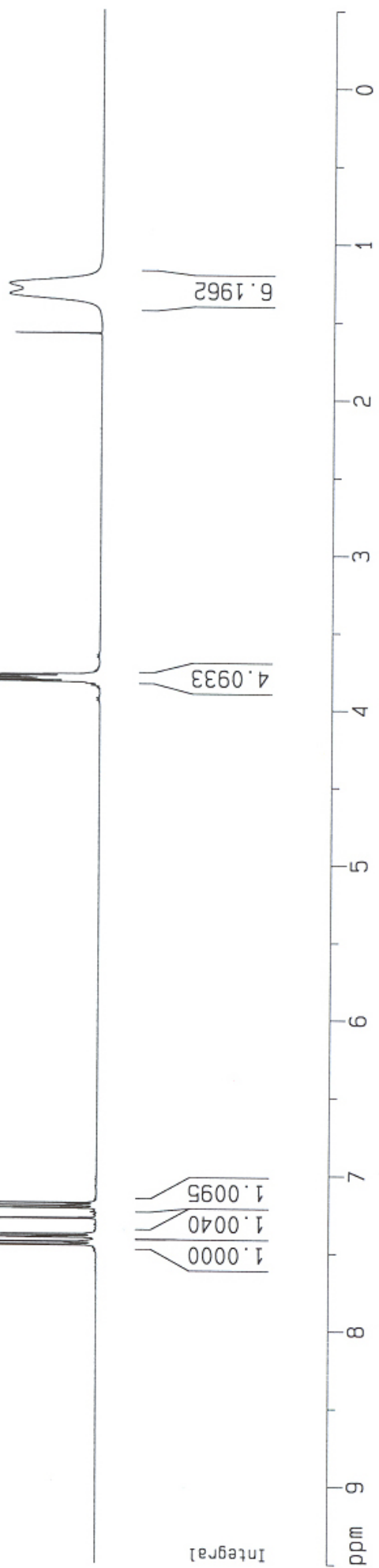

Figure S85: ${ }^{1} \mathrm{H}$ NMR of $\mathbf{3 5 c}$ 


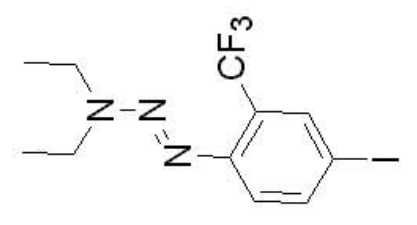

IE口 โI -

$618^{\circ} \nabla \leftarrow$

929 ट૪

เน6.6

$081^{\circ} \angle L$

$860^{\circ} \angle L$

GI8. $\angle L$

$\nabla I G^{\circ} \angle 8$

9L9.6I

$\nabla 99^{\circ} 61$

О8ह टट

IOI

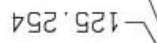

899. 9टा

$998^{\circ}$ વटा L

EG '9टा

$828^{\circ} \angle C I-$

8Eट जE T

86ट $9 \varepsilon \downarrow$

$\nabla G E^{\circ} \subseteq E \downarrow$

टID. GET

9L大比-

OSO $6 \mathrm{VI}$

wdd

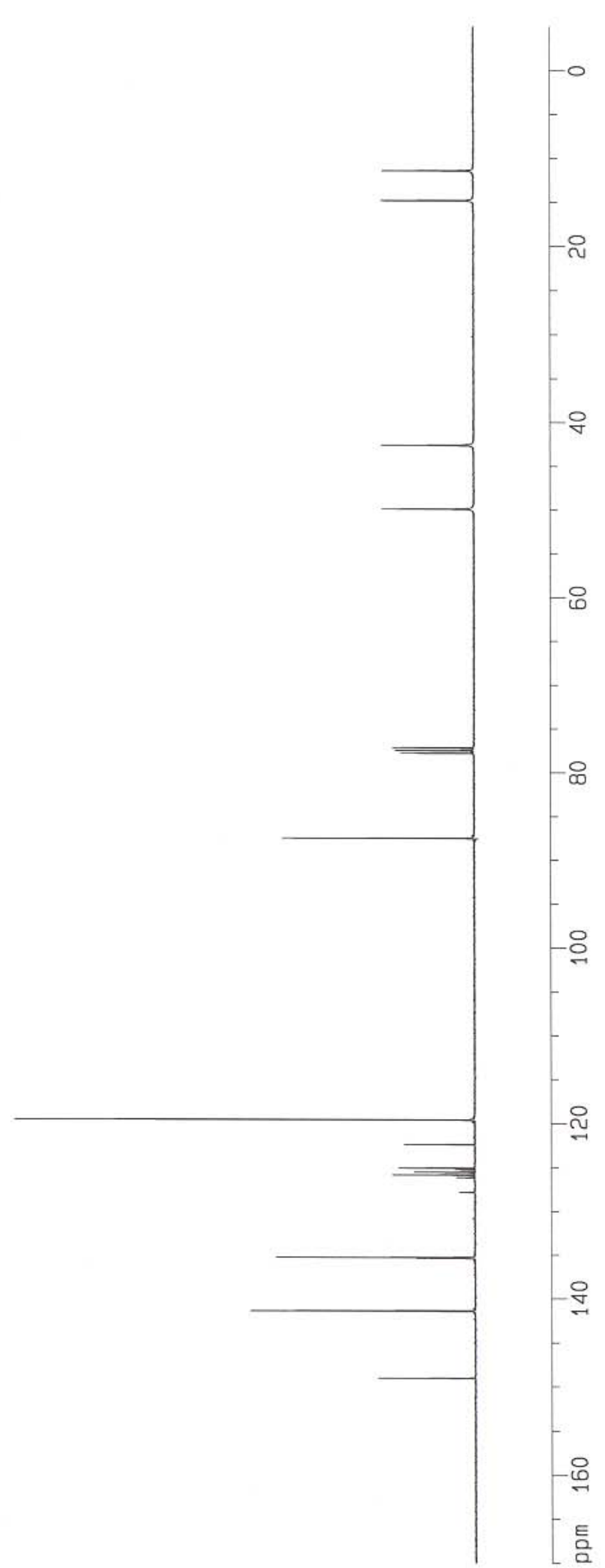

Figure S86: ${ }^{13} \mathrm{C}$ NMR of $\mathbf{3 5 d}$ 

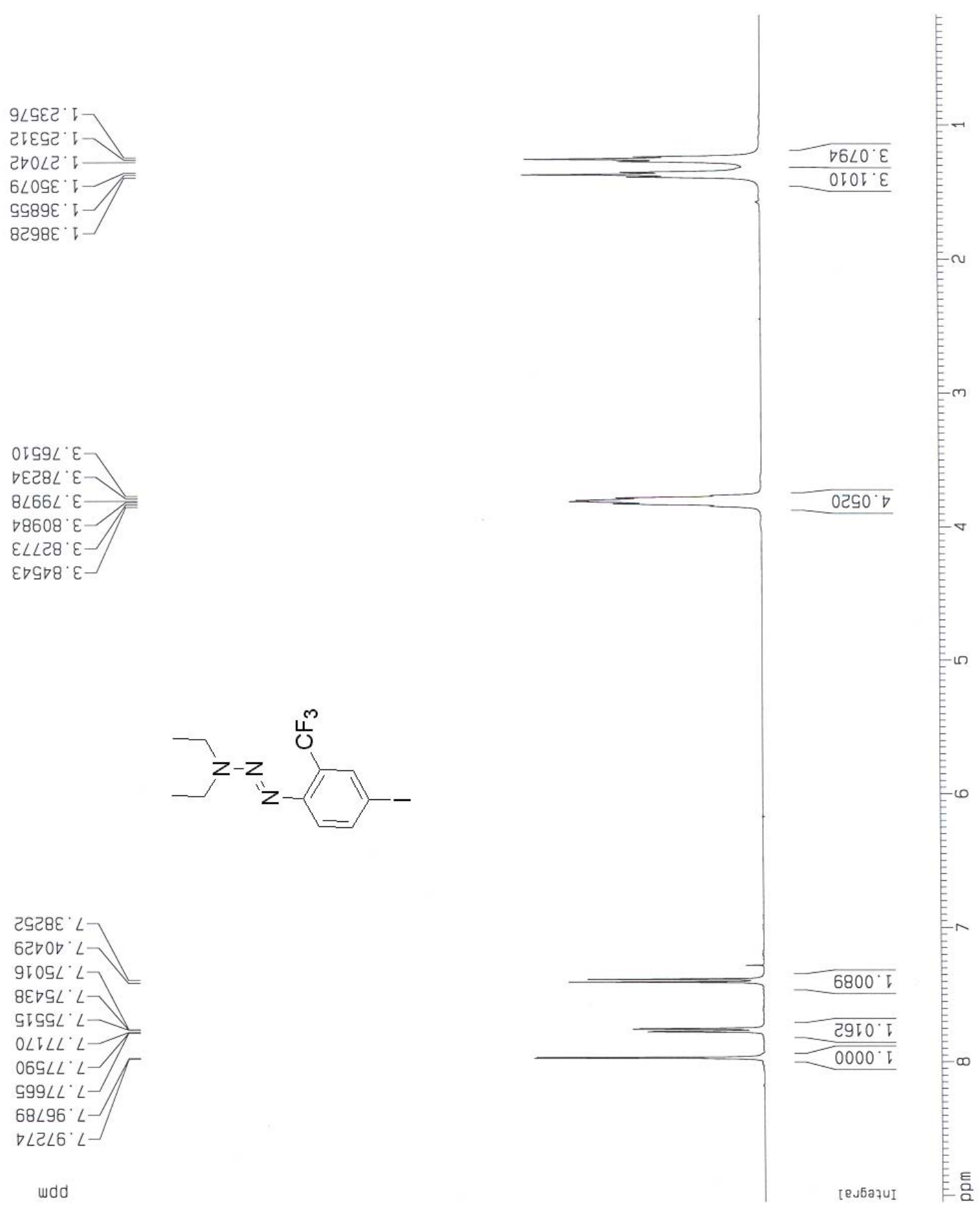

Figure S87: ${ }^{1} \mathrm{H}$ NMR of $\mathbf{3 5 d}$ 


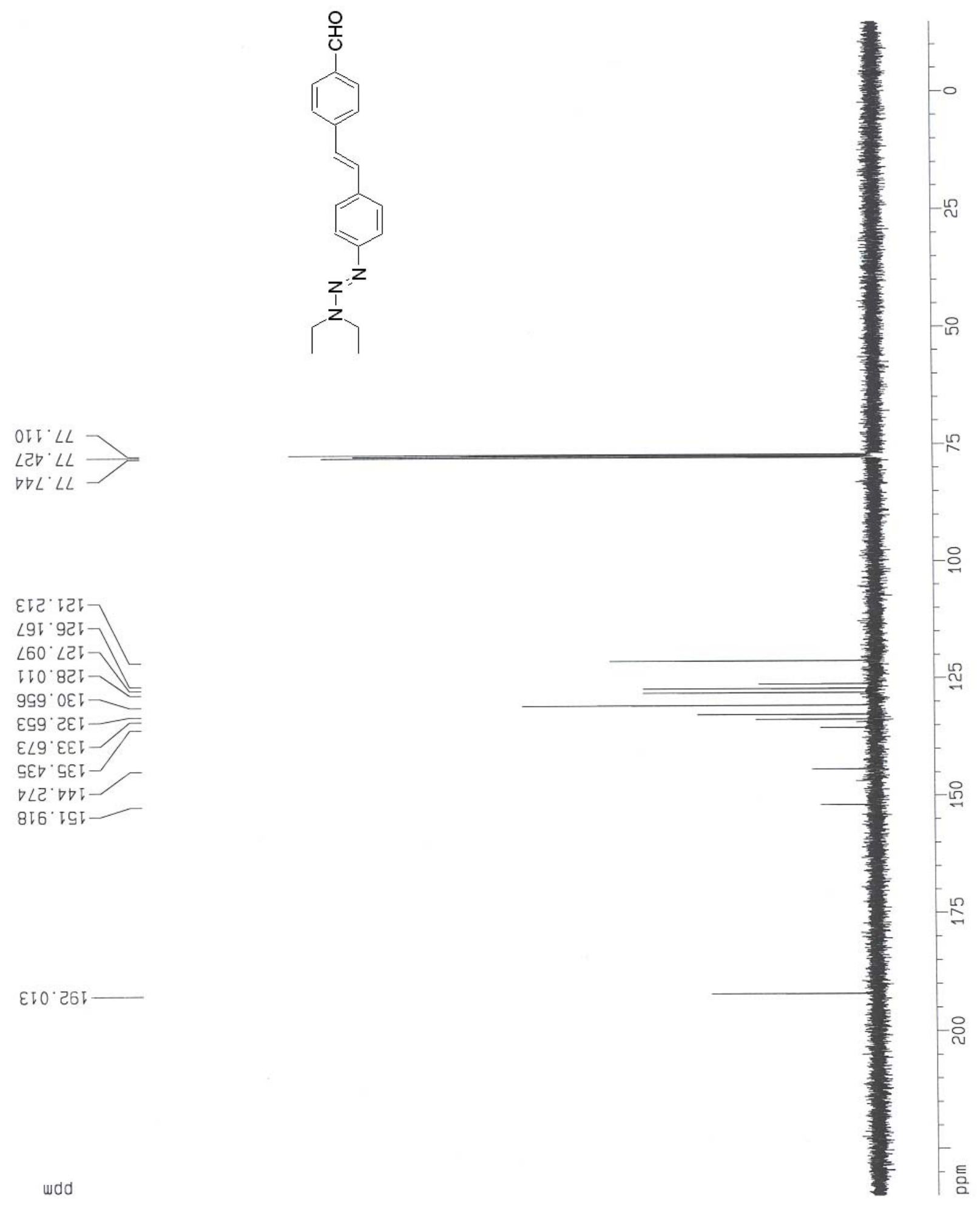

Figure S88: ${ }^{13} \mathrm{C}$ NMR of $\mathbf{3 6}$ 

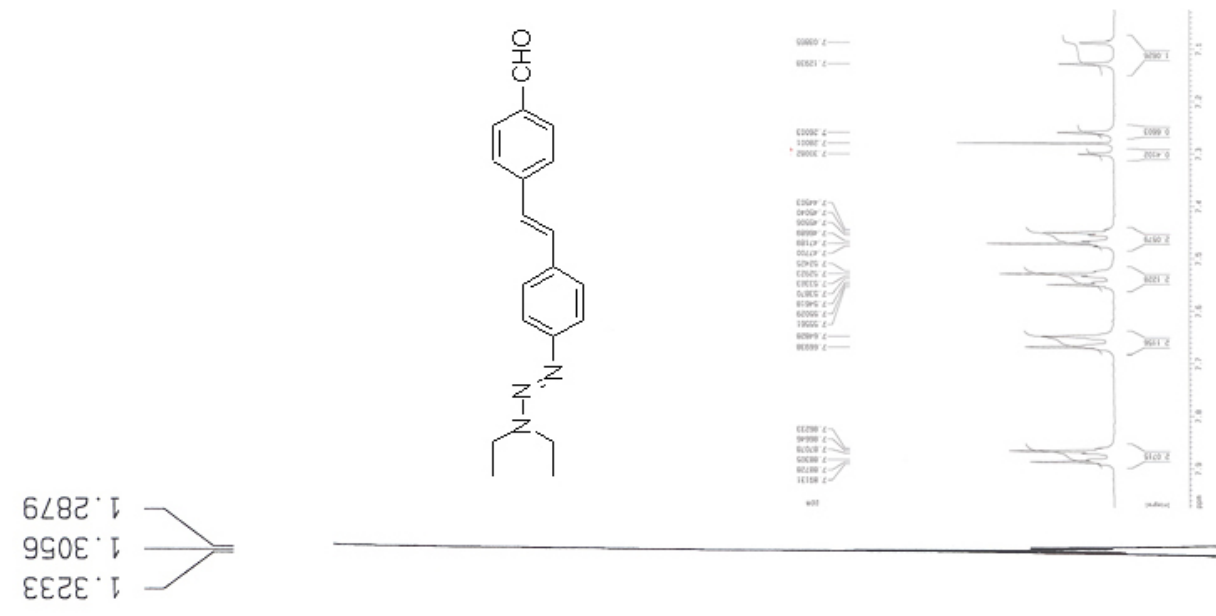

โ โ8 ${ }^{\prime} \varepsilon$

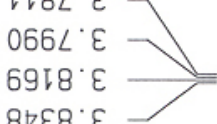

$8 \nabla \varepsilon 8^{\cdot} \varepsilon$

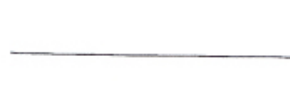

\section{$9880^{\circ} \mathrm{L}$}

จ6टा ' $L$

$0092^{\circ} L$

$0082^{\circ} L$

$800 \varepsilon^{\prime} L$

$\checkmark 0 \nabla^{\circ} L$

ISG) $\angle$

$699 \nabla^{\circ} L$

$6 I \angle \nabla^{\circ} \angle \longrightarrow$

टбटद ' $L$

8EहG $\angle$

हOSG $\angle$

$\varepsilon 8 \nabla 9^{\circ} \angle$

$\nabla 699^{\circ} \angle$

$5998^{\circ} \mathrm{L}$

$80 \angle 8^{\circ} \angle$

'E88' $\angle$

$\varepsilon \angle 88^{\circ} \angle$

$\mathrm{\nabla 00} \mathrm{Or}$
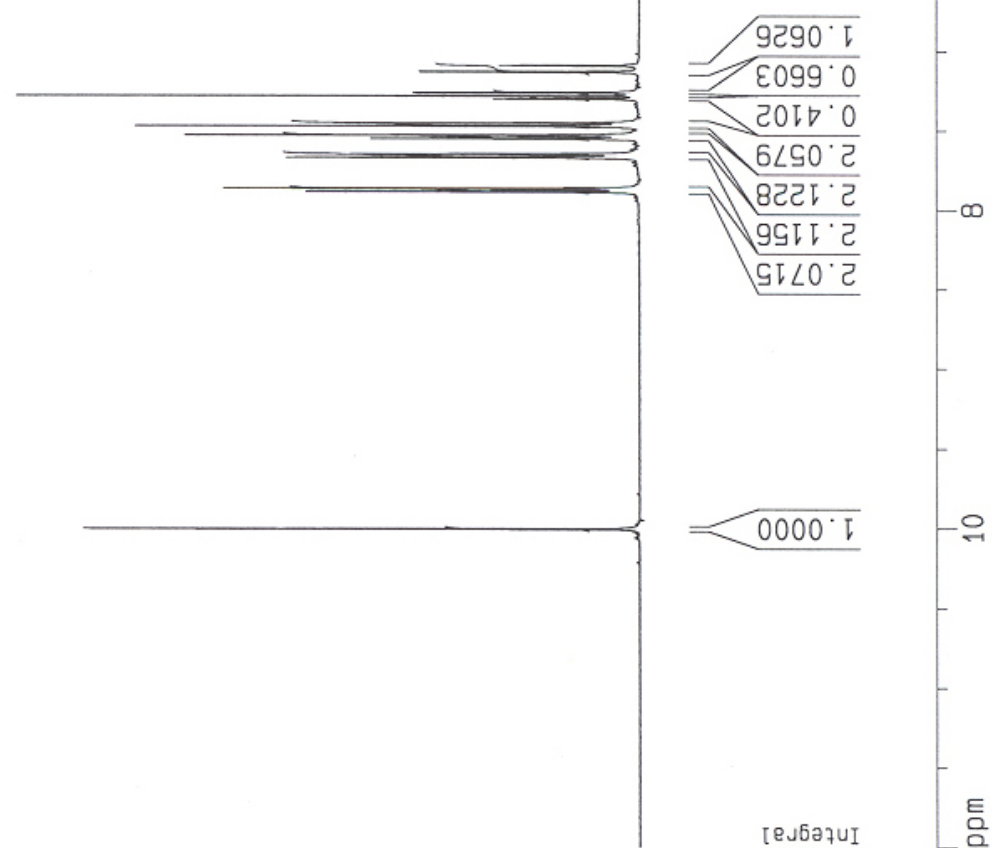

Figure S89: ${ }^{1} \mathrm{H}$ NMR of $\mathbf{3 6}$ 
GLC'GL

$\mathrm{S} 8 L \mathrm{GI} \longrightarrow$

$690^{\circ} 92$

$90 己^{\circ} \nabla \varepsilon$

I8I' LL

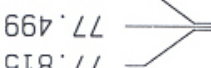

SI8. $\angle L$

टट8.66

ट81 เᄃा

680. $951-$

$\angle 16{ }^{\circ} \mathrm{Cट}$

टLI $\angle 2$

0टટ * $\angle$ I

$88 \varepsilon^{\circ} \angle 2 \downarrow$

II $\angle 2 \downarrow$

$6 \angle I 621$

$899^{\circ} 0 \varepsilon \downarrow$

¿GG $\nabla E[-$

$\angle 89.9 E[-/ / F$

$006 \cdot 9 \varepsilon \downarrow-]$

$\angle O L^{\circ} \angle E I-$

$606^{\circ} 6 \varepsilon[-$

$\nabla 8<$ เレレ

6เ9'จナレ]

G6ट. โG -

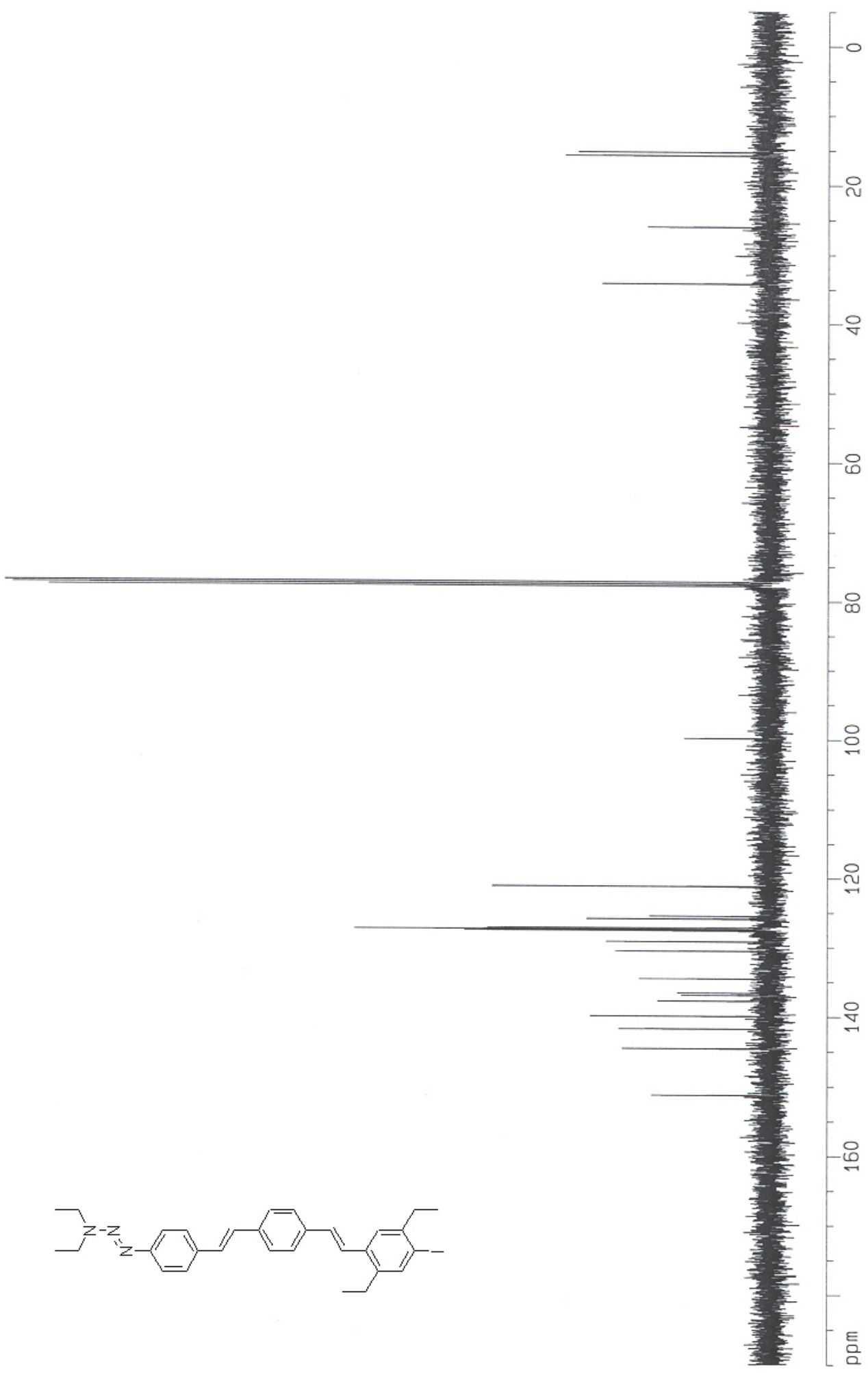

wdd

Figure S90: ${ }^{13} \mathrm{C}$ NMR of $\mathbf{3 8}$ 


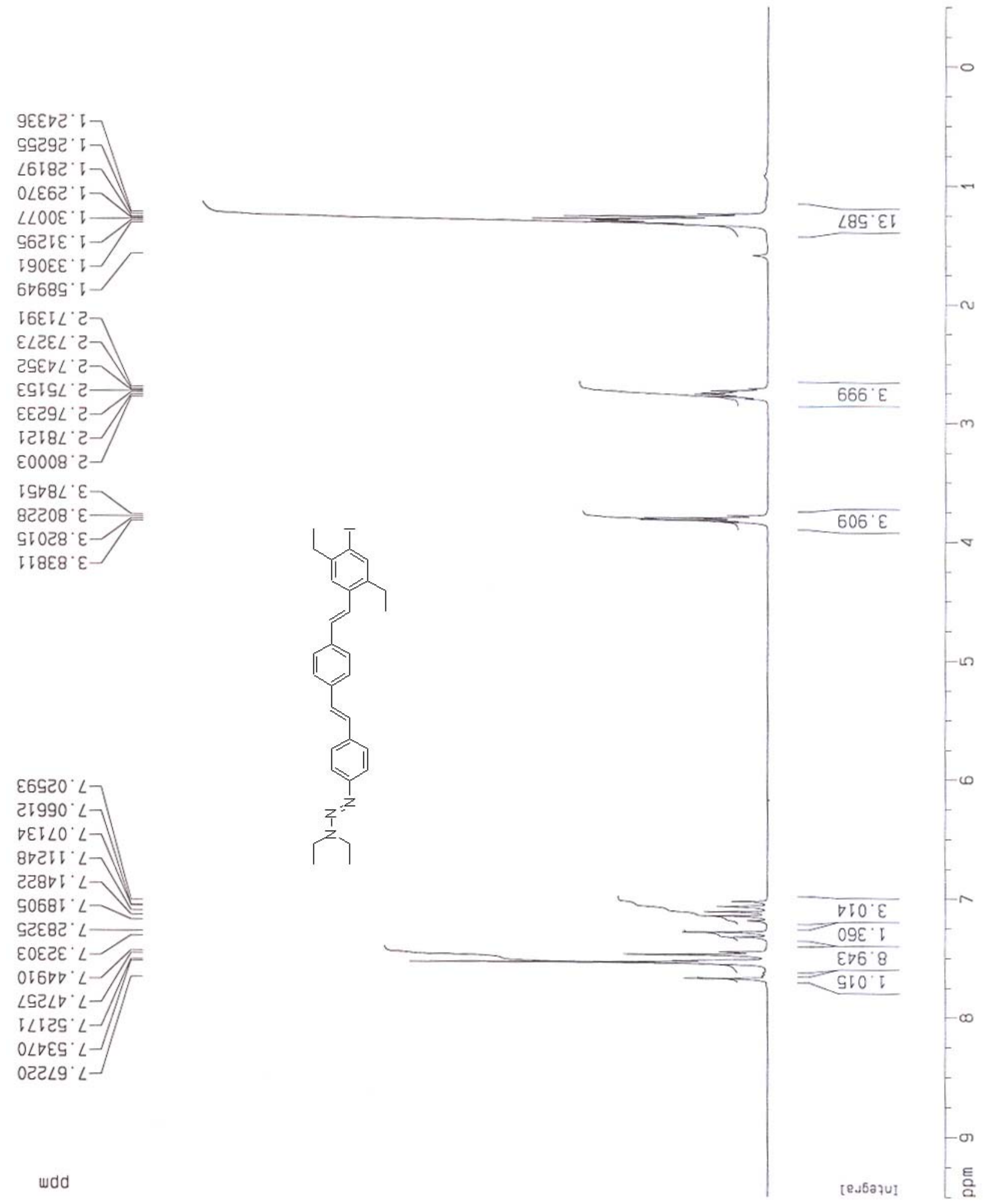

Figure S91: ${ }^{1} \mathrm{H}$ NMR of $\mathbf{3 8}$ 
$\nabla 05 \cdot 9$ I

$8 \tau \cdot 9 \tau \longrightarrow$

$66 L^{\circ} 92$

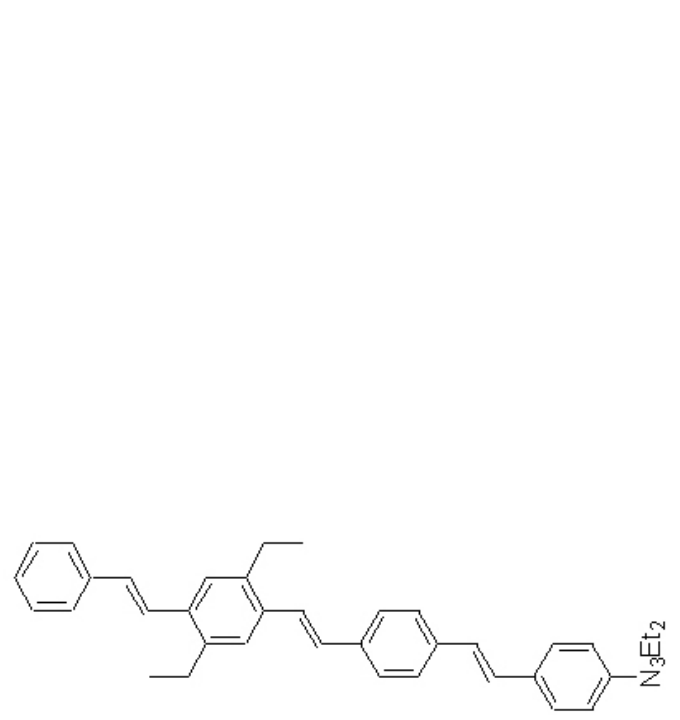

I8 ' $\angle L$

$66 \nabla^{\circ} \angle L$

$\angle I 8^{\circ} \angle L$

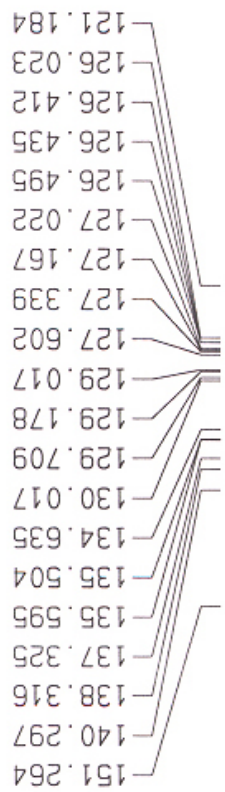

wdd

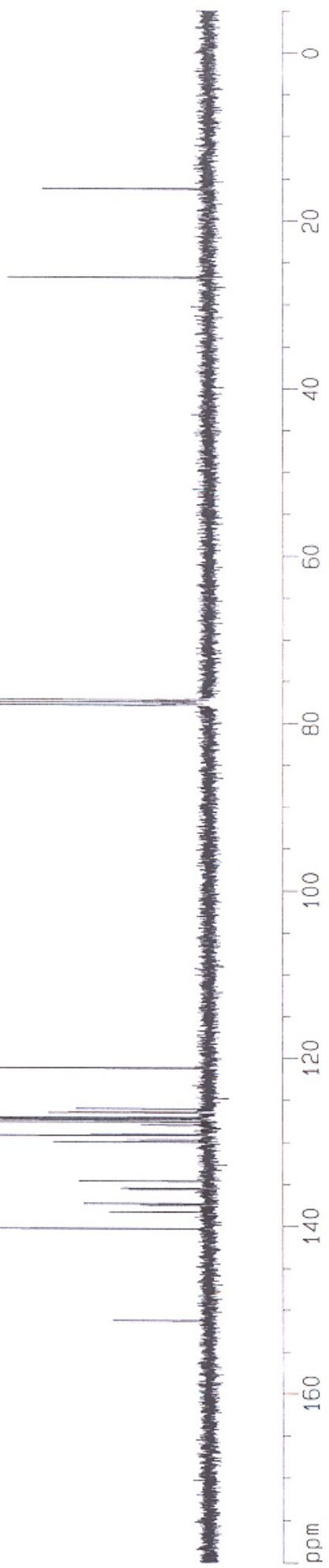

Figure S92: ${ }^{13} \mathrm{C}$ NMR of 39 


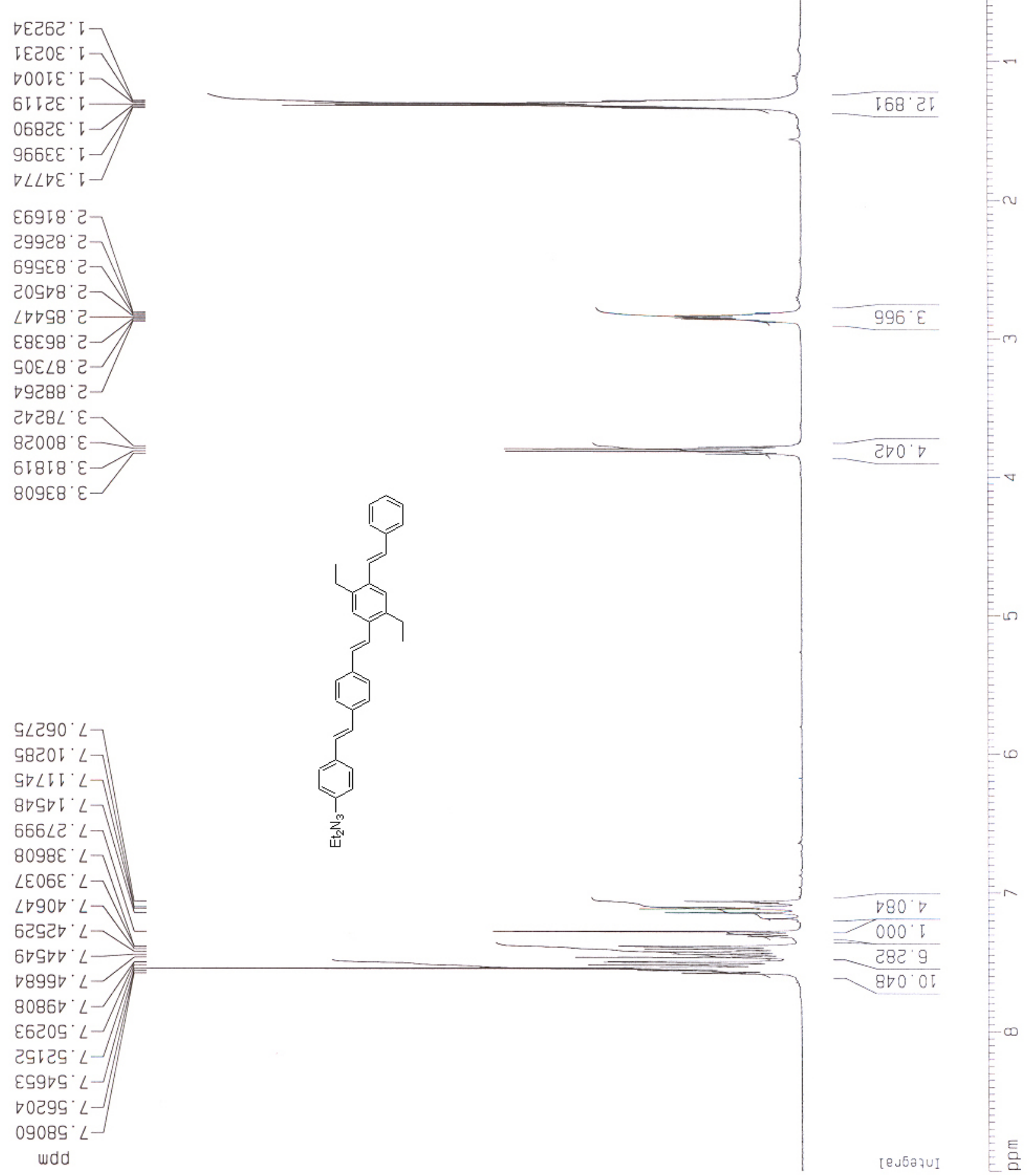

Figure S93: ${ }^{1} \mathrm{H}$ NMR of 39 

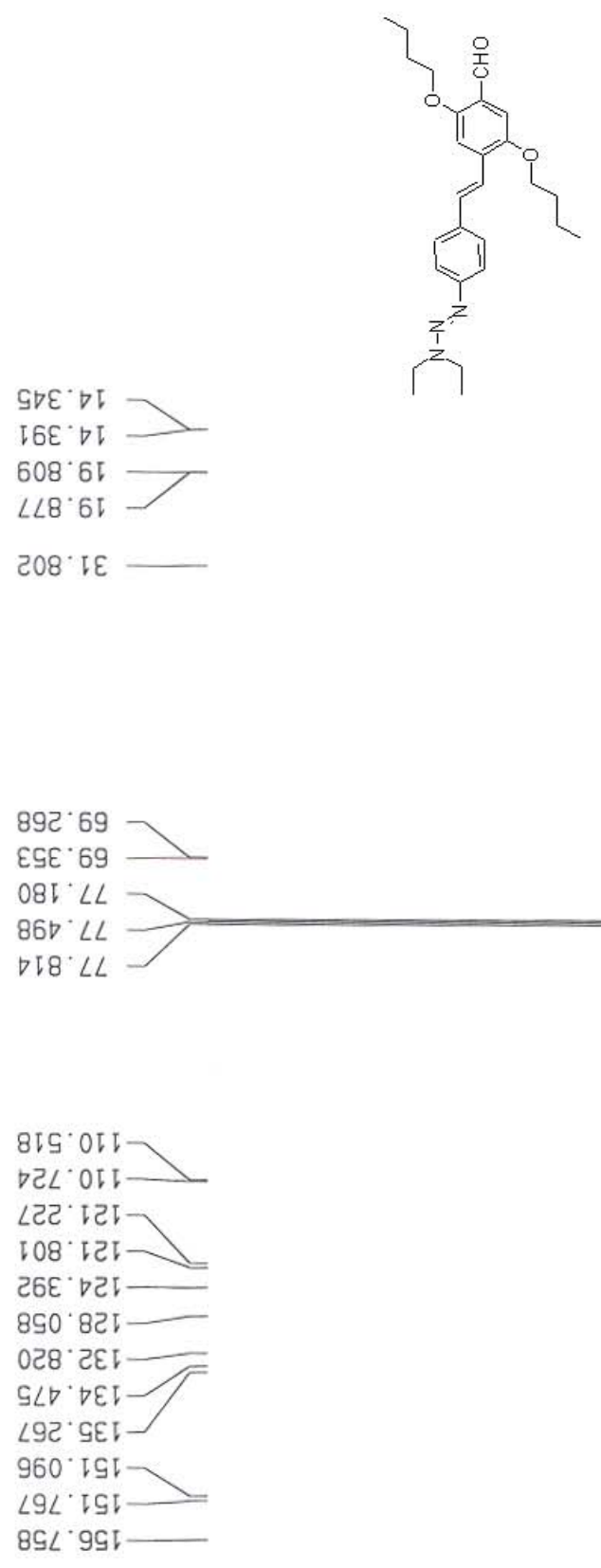

$\varepsilon \varepsilon 9^{\circ} 68 \mathrm{I}$

wdd

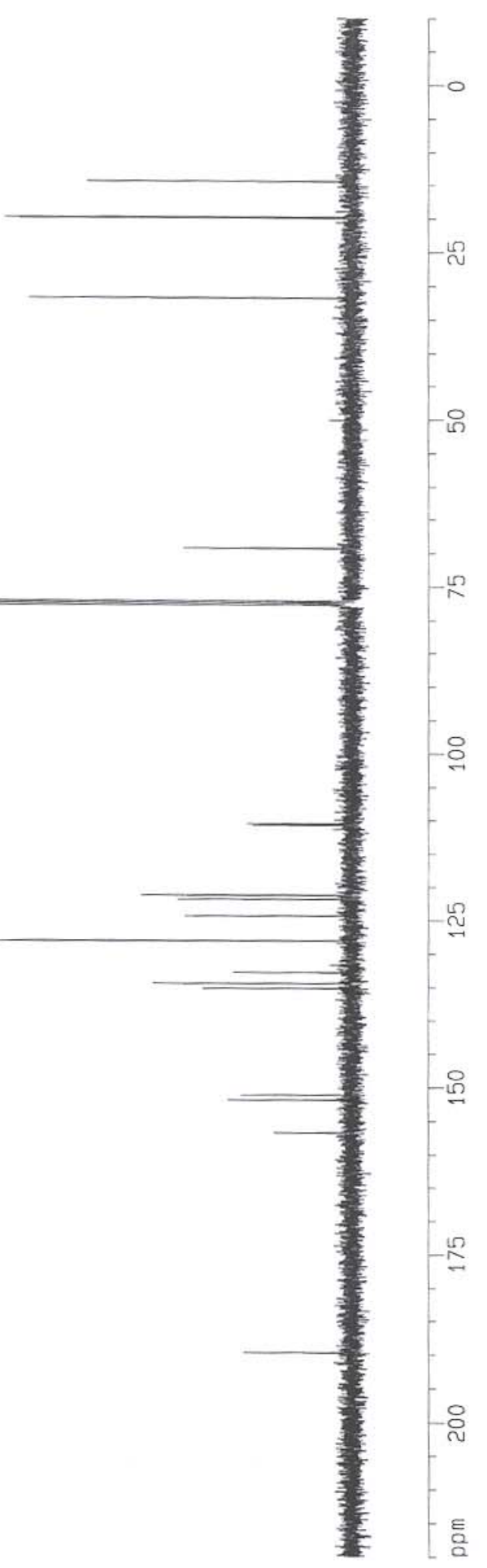

Figure S94: ${ }^{13} \mathrm{C}$ NMR of $\mathbf{4 0}$ 


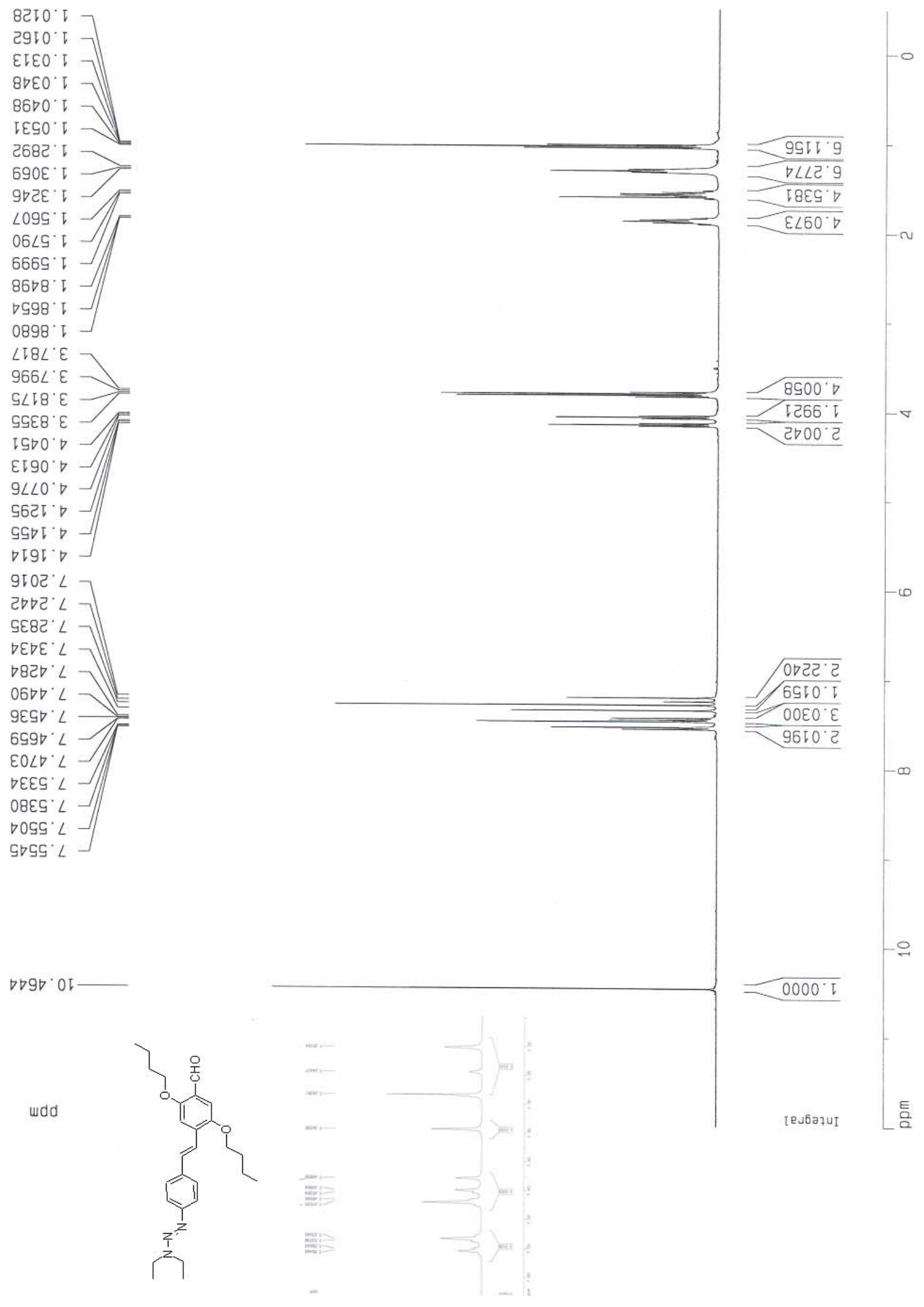

Figure S95: ${ }^{1} \mathrm{H}$ NMR of $\mathbf{4 0}$ 


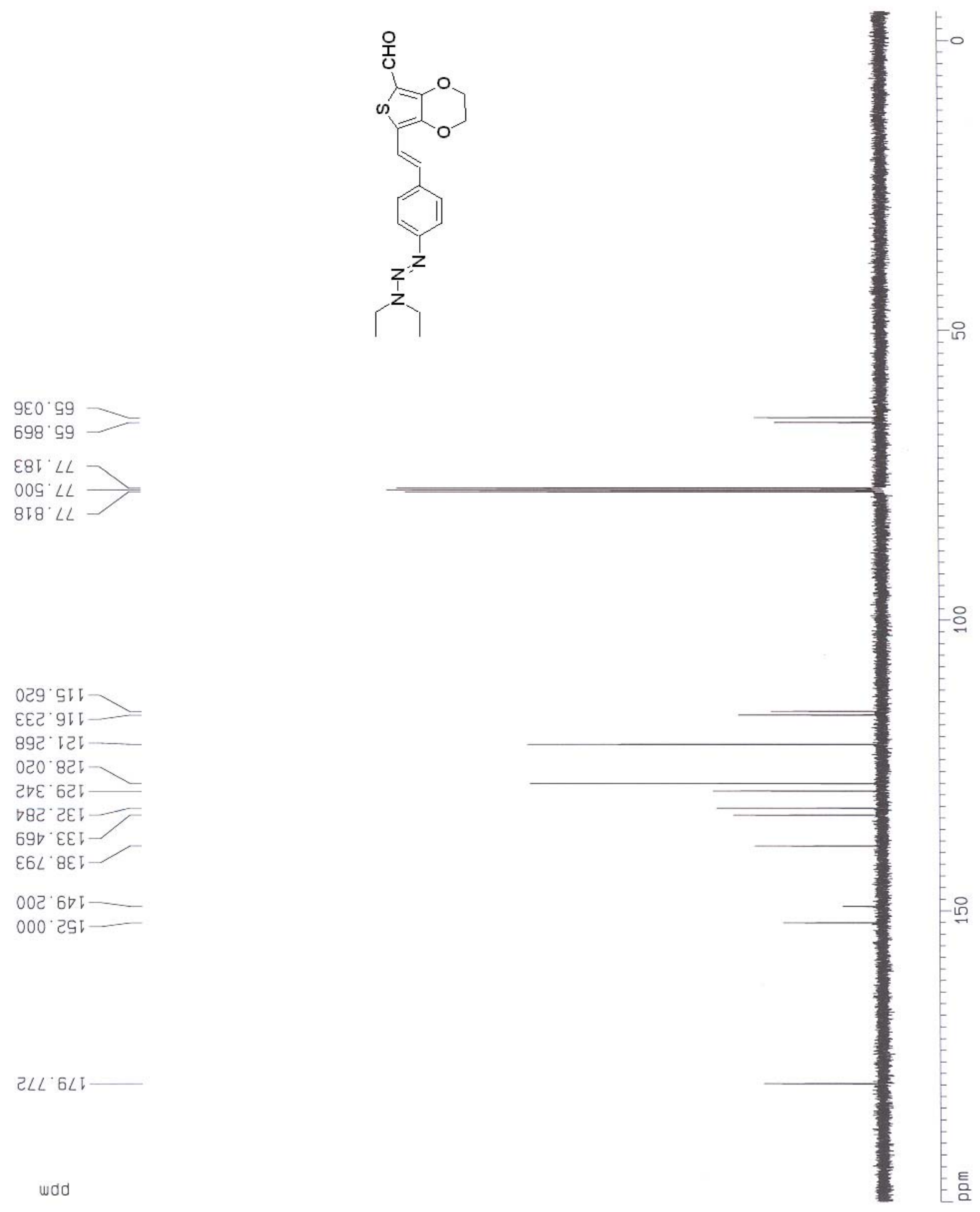

Figure S96: ${ }^{13} \mathrm{C}$ NMR of 41 

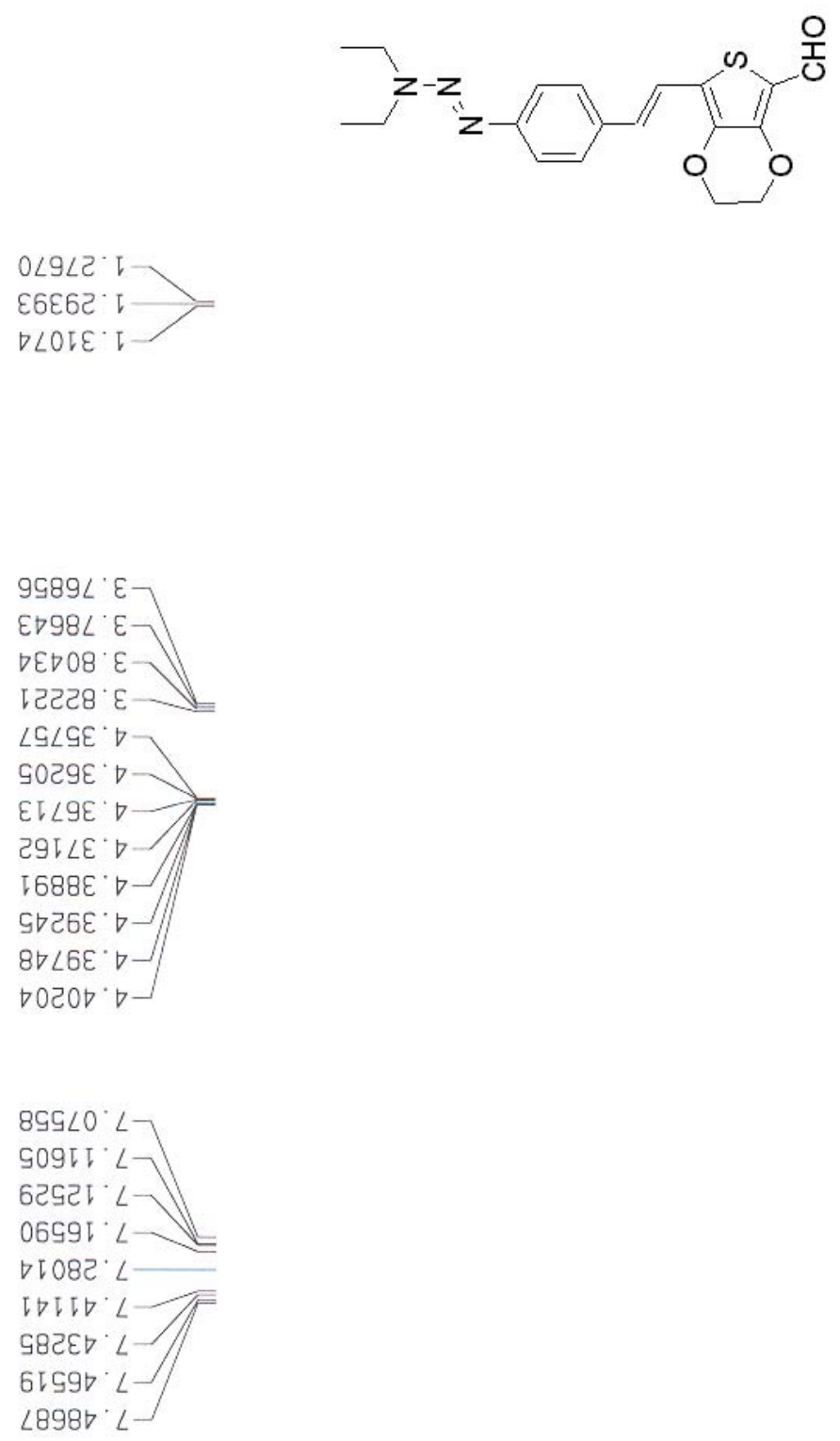

$\nabla \angle \nabla 68^{\circ} 6$

wdd

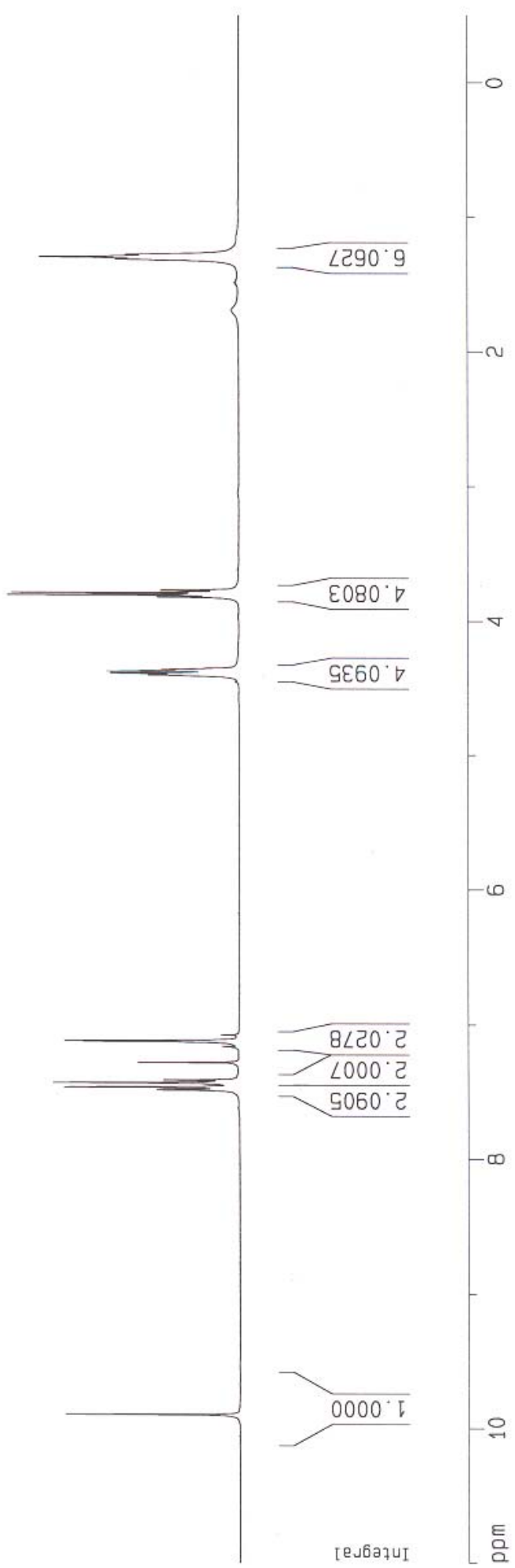

Figure S97: ${ }^{1} \mathrm{H}$ NMR of $\mathbf{4 1}$ 
$\varepsilon 66 \angle C^{*}$ ट9८6ट ट૪GIE SLC6० จा689.

โ $80 \angle L^{\circ} \varepsilon$ $\nabla 988 \angle \cdot \varepsilon$ $\nabla 9908^{\prime} \varepsilon$ โร口ट8' $\varepsilon-$ 606टE'
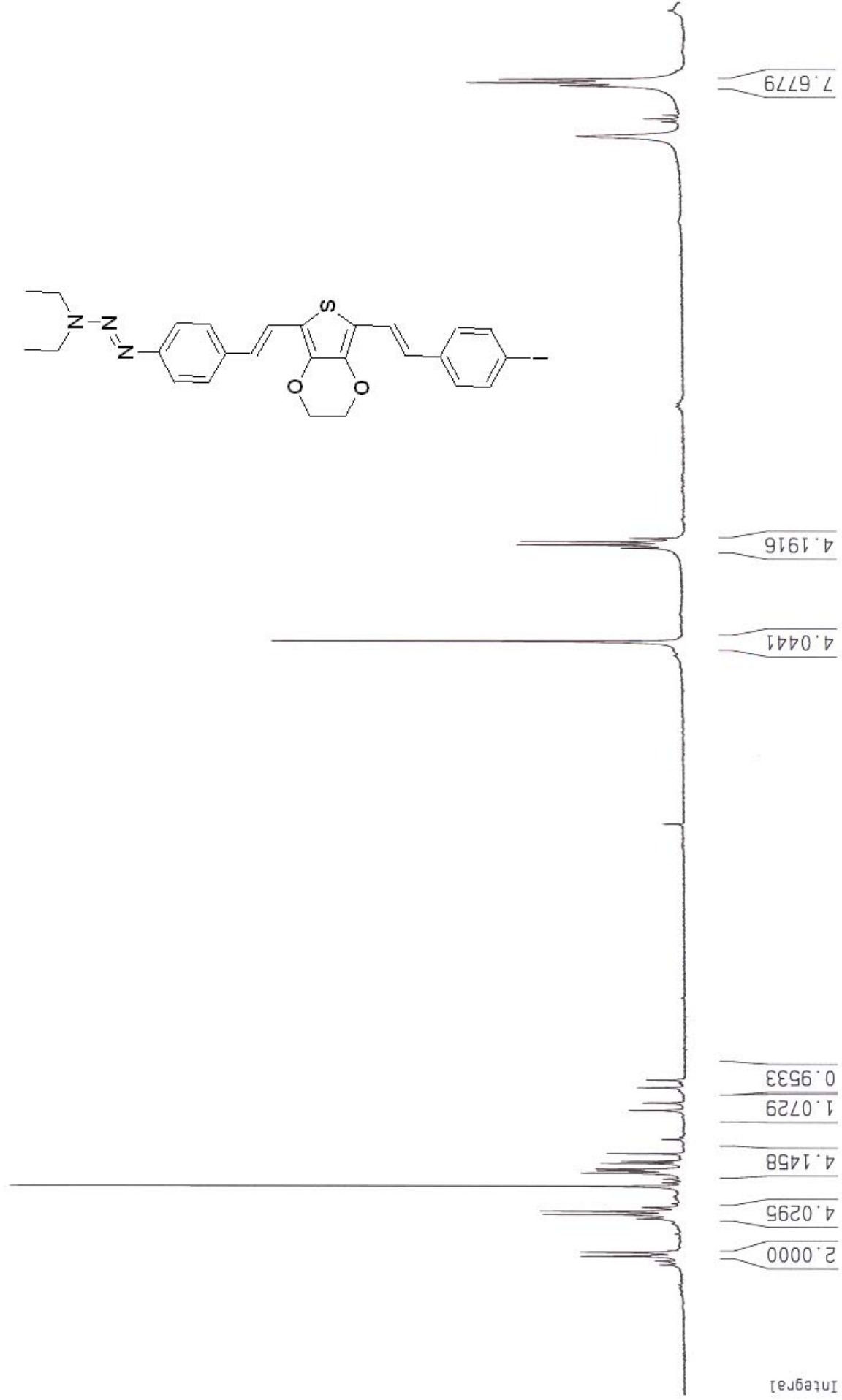

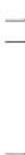
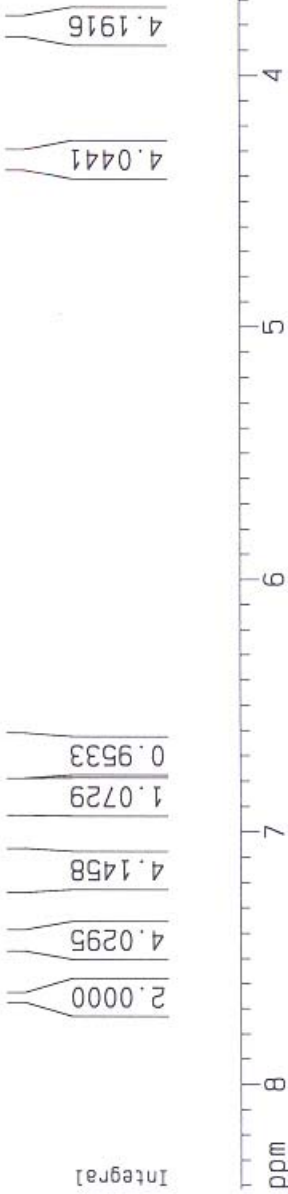

टOES $\angle \cdot 9$ 069ع8. $829 \angle 8 \cdot 9-$ 0960 Г. โ860 โ $\angle \neg$ 9819 9986 I ' $L$ G020ट ' $\angle \checkmark \backslash$ 89DIट 88в8ट ' $L$ हโ66ะ โ60ट७ $\angle$ $69 \angle E \nabla^{\circ} \angle$ 8965 $\angle$ $90 \varepsilon \nabla 9^{\circ}<-$ $29 \angle \nabla 9^{\circ} \angle-$ 8นจ99 $\angle$ wdd

Figure S98: ${ }^{1} \mathrm{H}$ NMR of $\mathbf{4 2}$ 


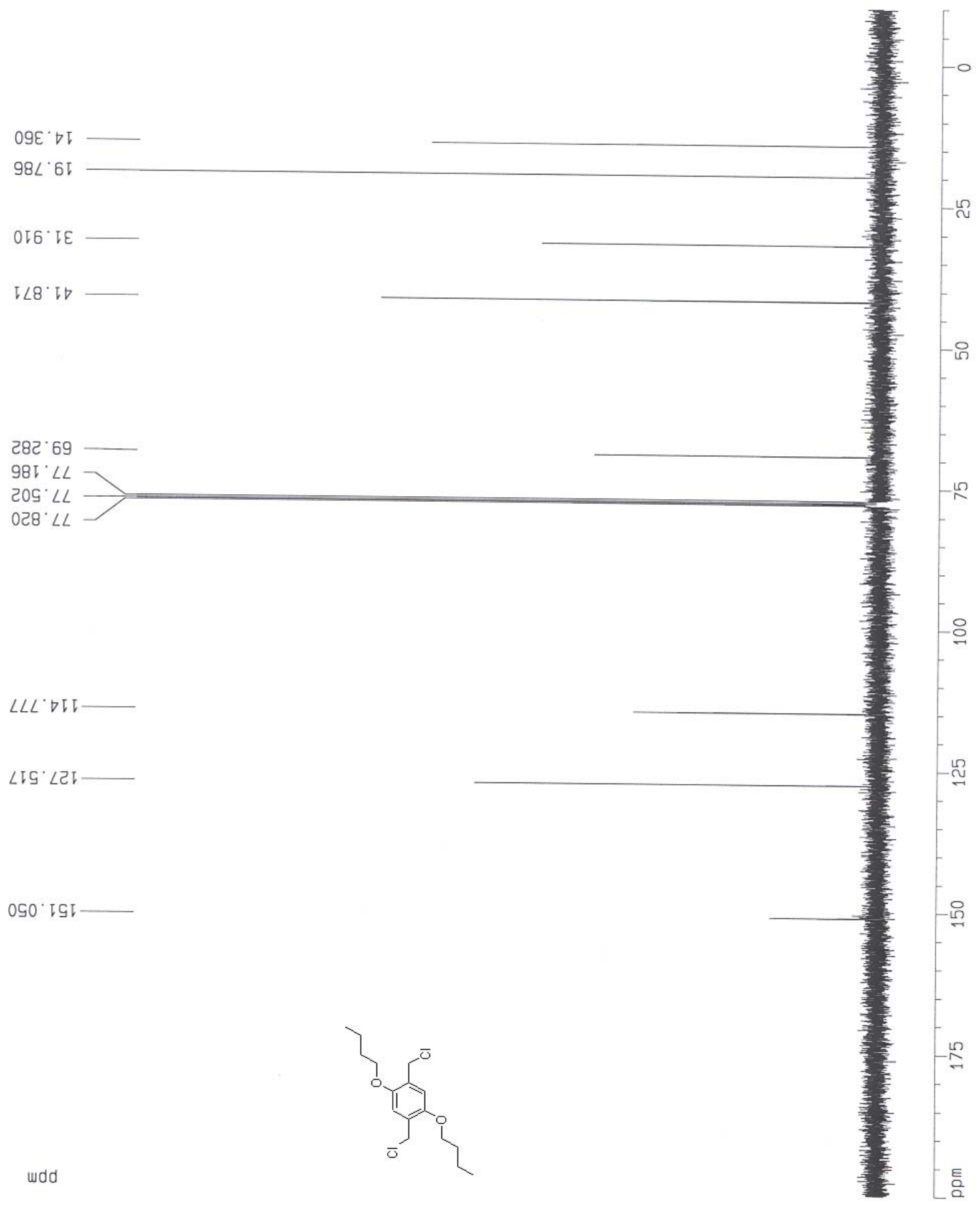

Figure S99: ${ }^{13} \mathrm{C}$ NMR of $\mathbf{4 3}$ 


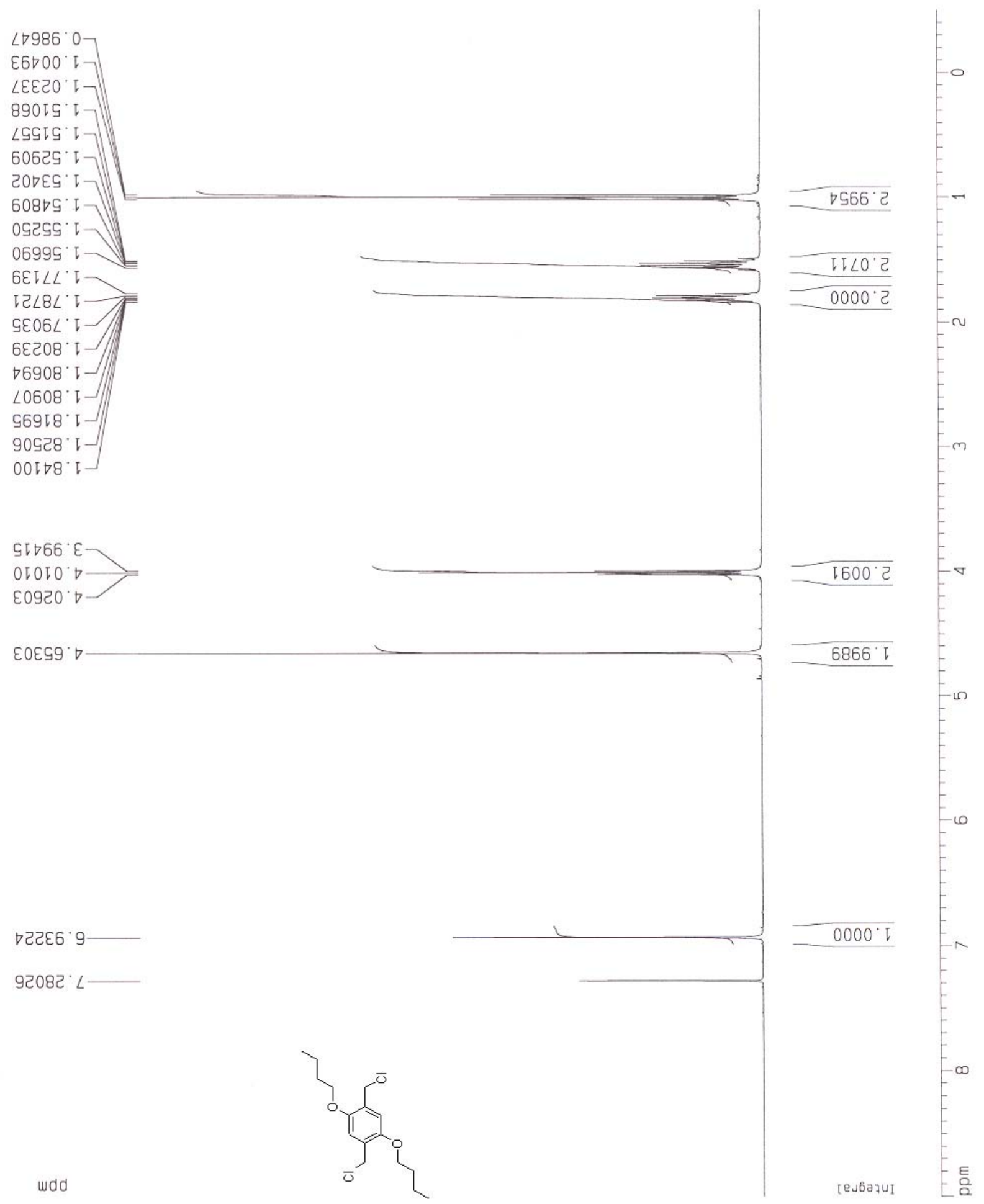

Figure S100: ${ }^{1} \mathrm{H}$ NMR of $\mathbf{4 3}$ 


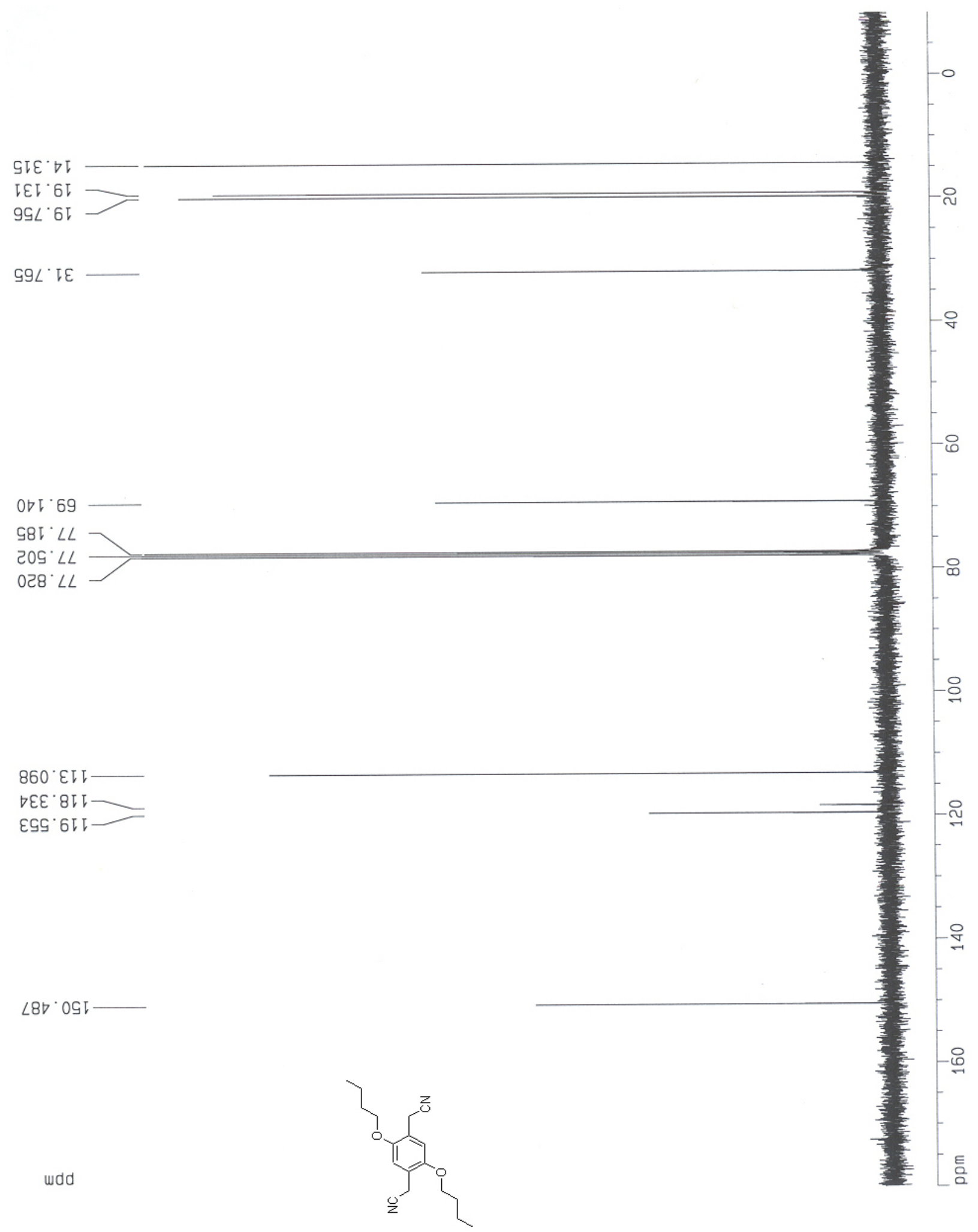

Figure S101: ${ }^{13} \mathrm{C}$ NMR of 44 


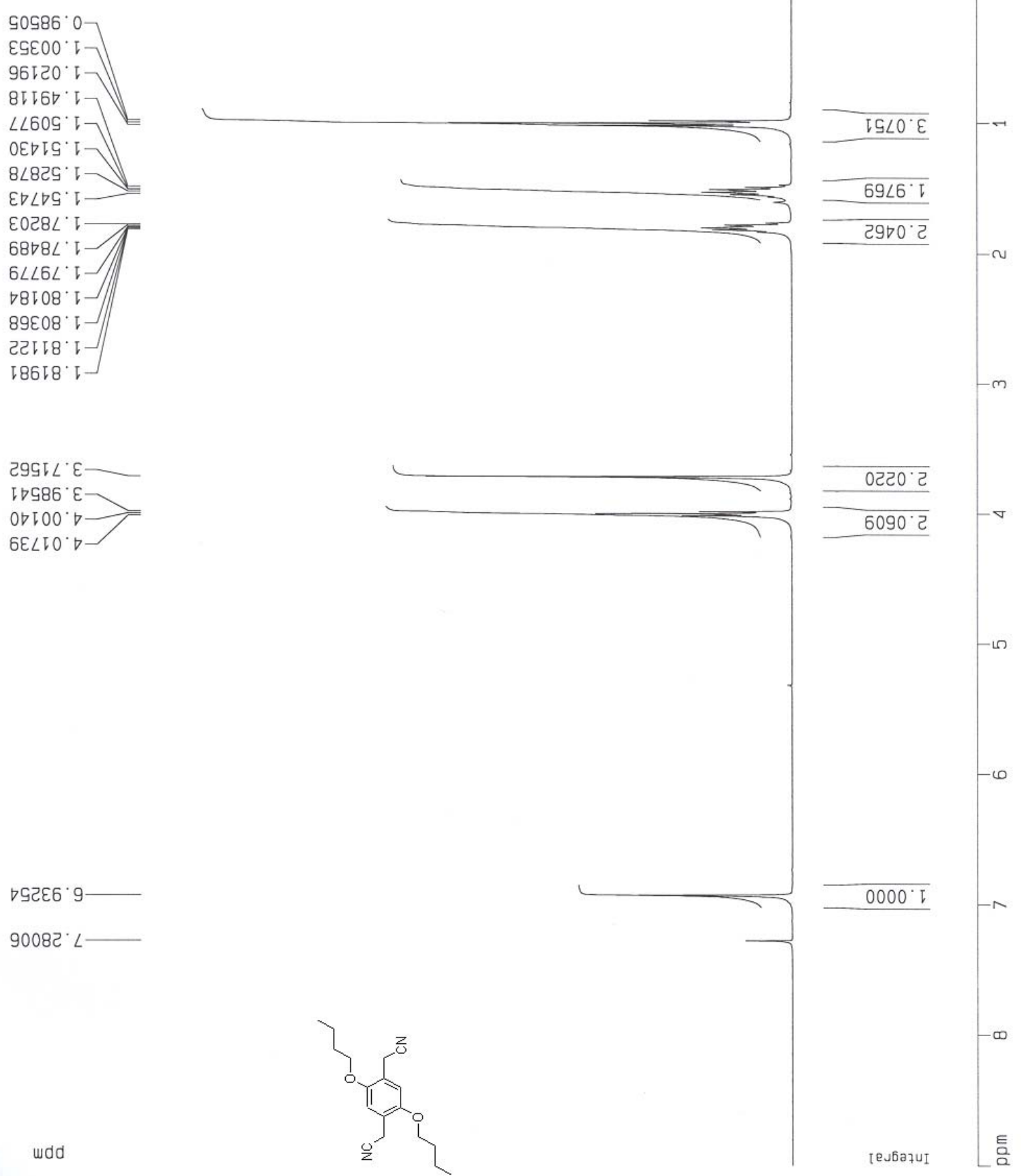

Figure S102: ${ }^{1} \mathrm{H}$ NMR of $\mathbf{4 4}$ 
$\nabla O G^{\circ} \nabla \downarrow$

घE६ $6 \mathrm{I}$

$\varepsilon 88^{\circ}$ เE

520. 99

$099^{\circ} 99$

$6 \nabla L \cdot 69$

$08 I^{\circ} \angle L$

$86 b^{\circ} \angle L$

$00 L^{\circ} \angle L$

$\checkmark\left\lceil 8^{\circ} L L\right.$

IL8. TOL

$\nabla \angle D^{\circ} \mathrm{SO}$

५GE $\nabla \downarrow \tau$

$0 Z \angle \circ \square \square$

69大.6I

$\nabla 6{ }^{\circ} \nabla 21$

$\triangleright 80^{\circ} \mathrm{GE}$

$\nabla \angle 8^{\circ} \sqcup \nabla L$

$608^{\circ} \nabla \nabla \sqcap-$

GEO โG

wdd
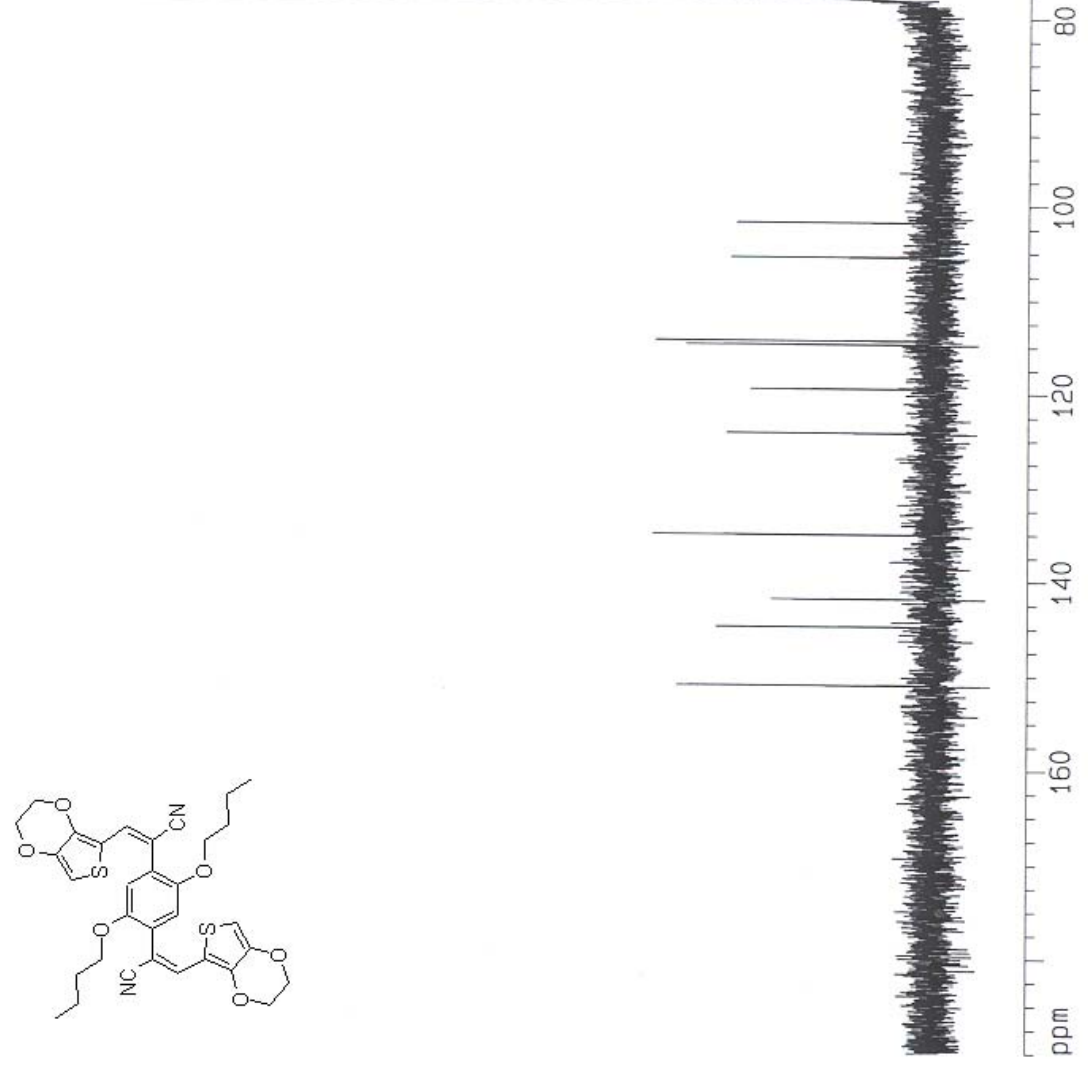

Figure S103: ${ }^{13} \mathrm{C}$ NMR of $\mathbf{4 5}$ 


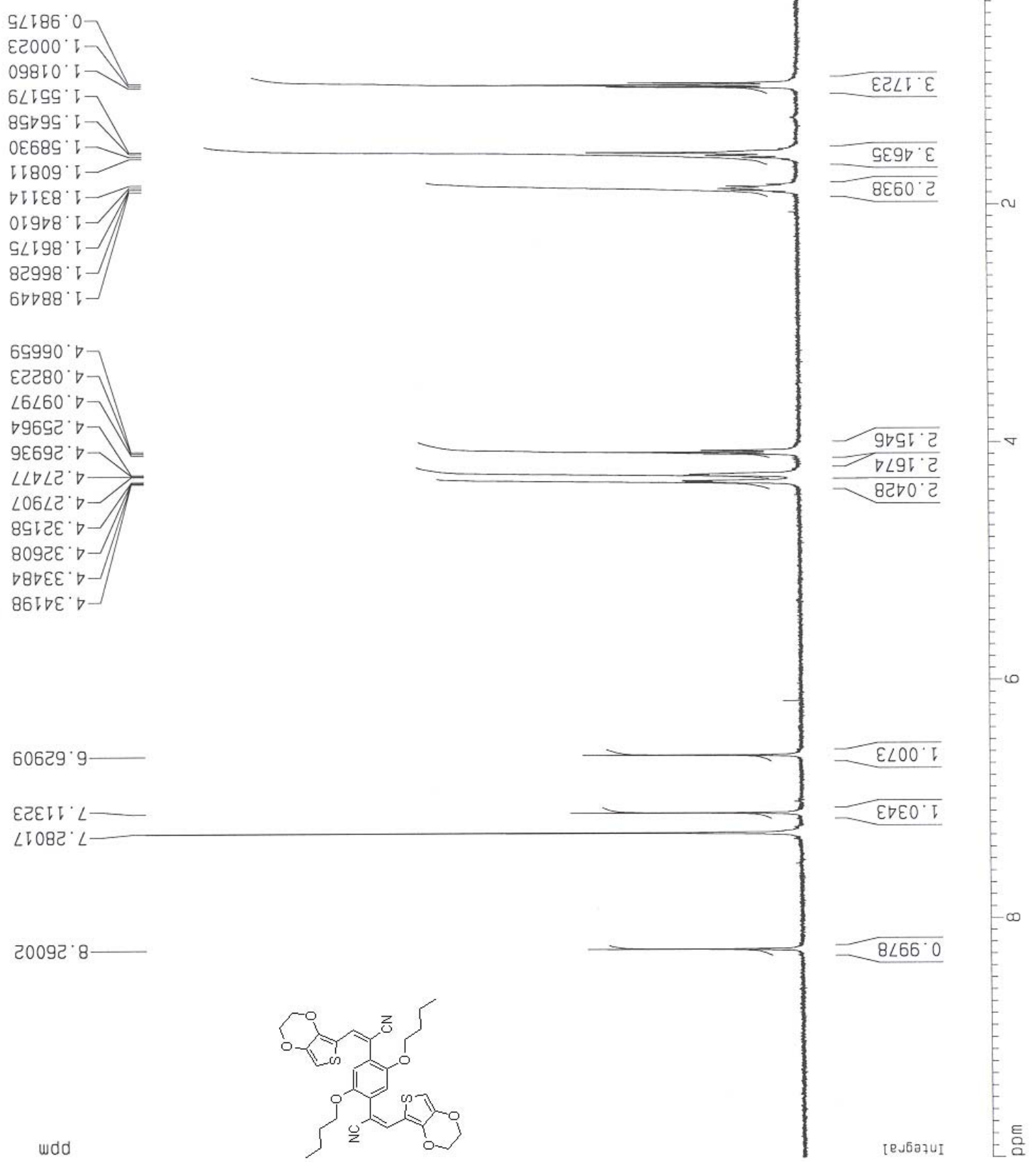

Figure S104: ${ }^{1} \mathrm{H}$ NMR of $\mathbf{4 5}$ 
$\nabla \nabla C^{\prime} \angle L$

$009^{\circ} \angle L$

$\nabla G L^{\circ} L L$

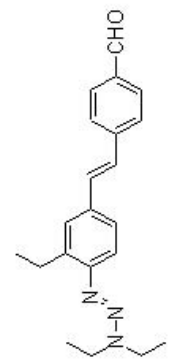

ICO $\angle I T$

909 द्रा-

S0L 921

$\nabla 06.951$

602.825

$\nabla \nabla \nabla^{\circ} O E \downarrow-$

$\angle G L$ टEI

ट૪૬' દEઈ

I6I GE

$\checkmark$ ऽ $6 E \downarrow$

$88 V^{\circ} \nabla \nabla L$

$080^{\circ} 6 \nabla 5-$

$9 \nabla L^{\circ}$ เ

wdd

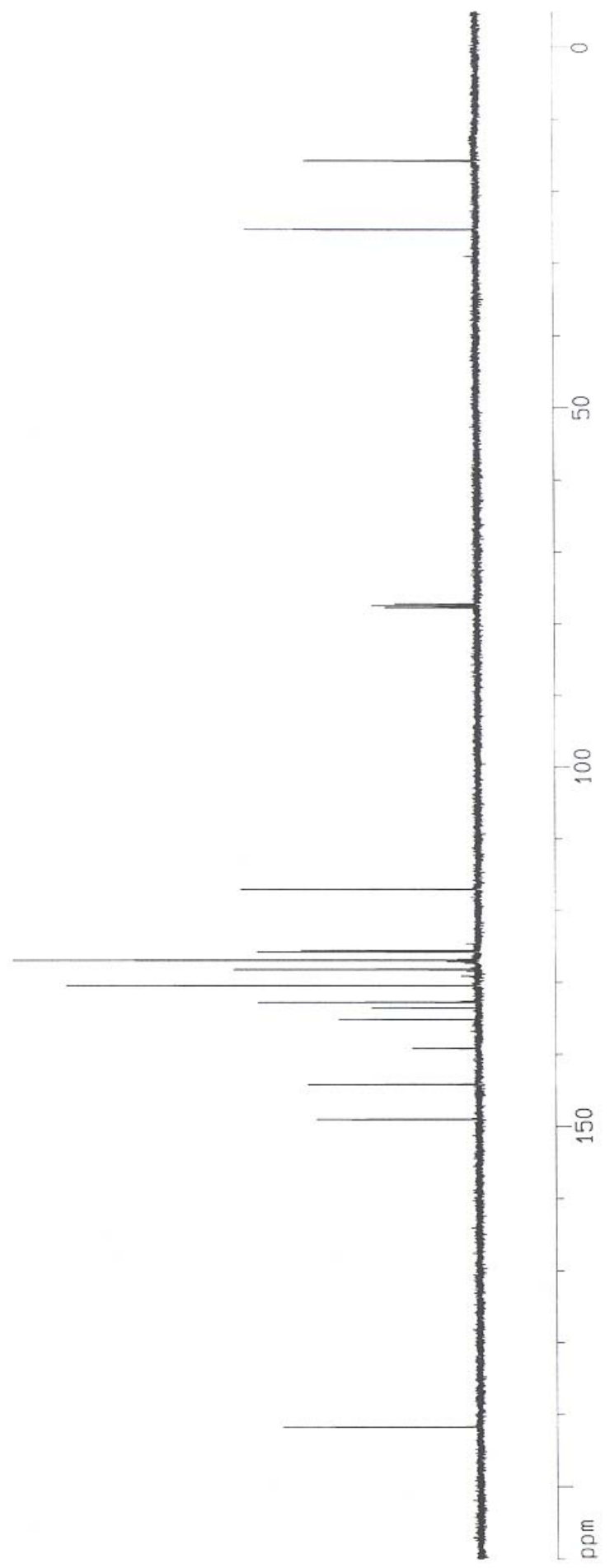

Figure S105: ${ }^{13} \mathrm{C}$ NMR of $\mathbf{4 6}$ 


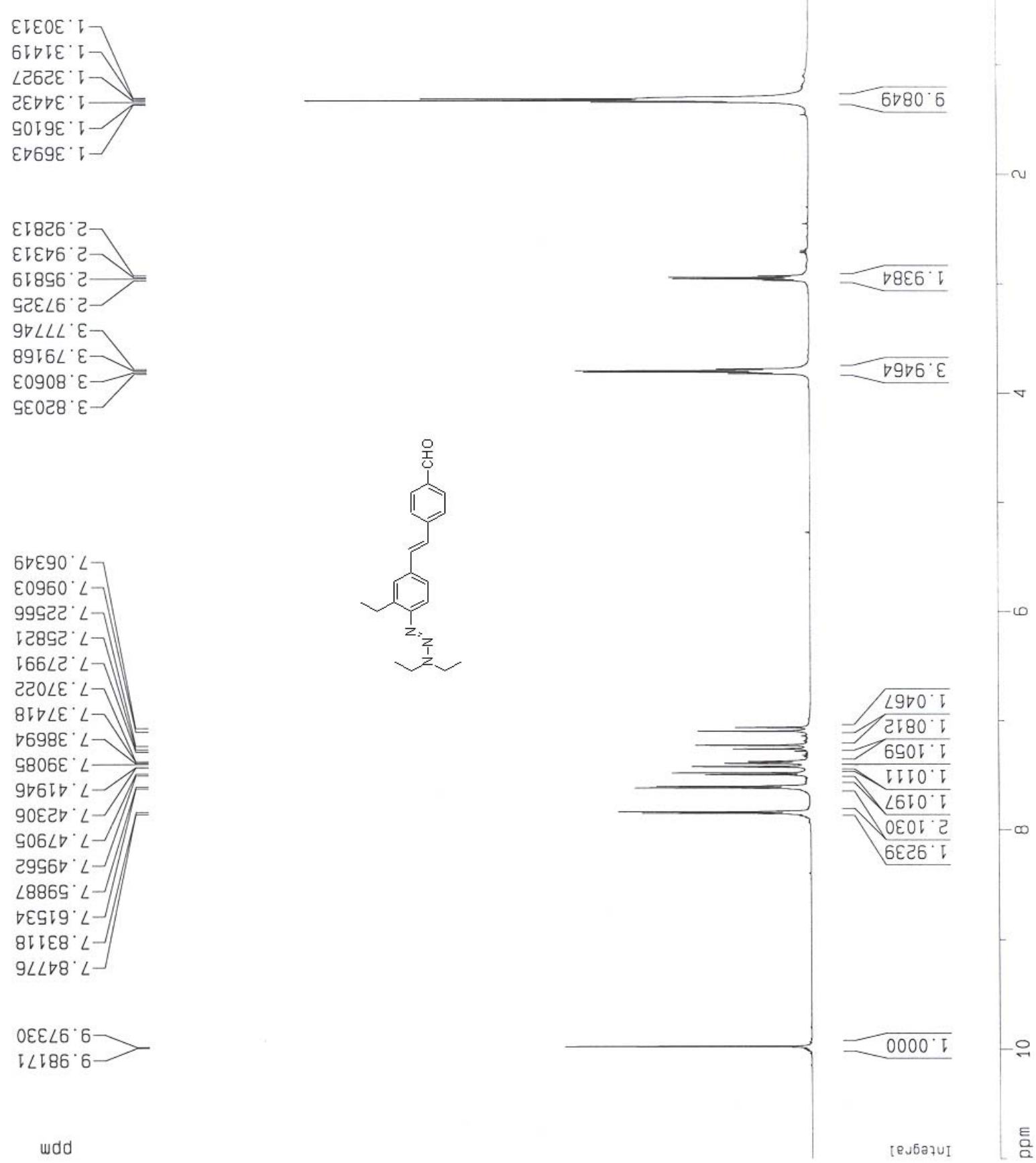

Figure S106: ${ }^{1} \mathrm{H}$ NMR of 46 
$\triangleright 96^{\circ} 91$

$809^{\circ} 92$

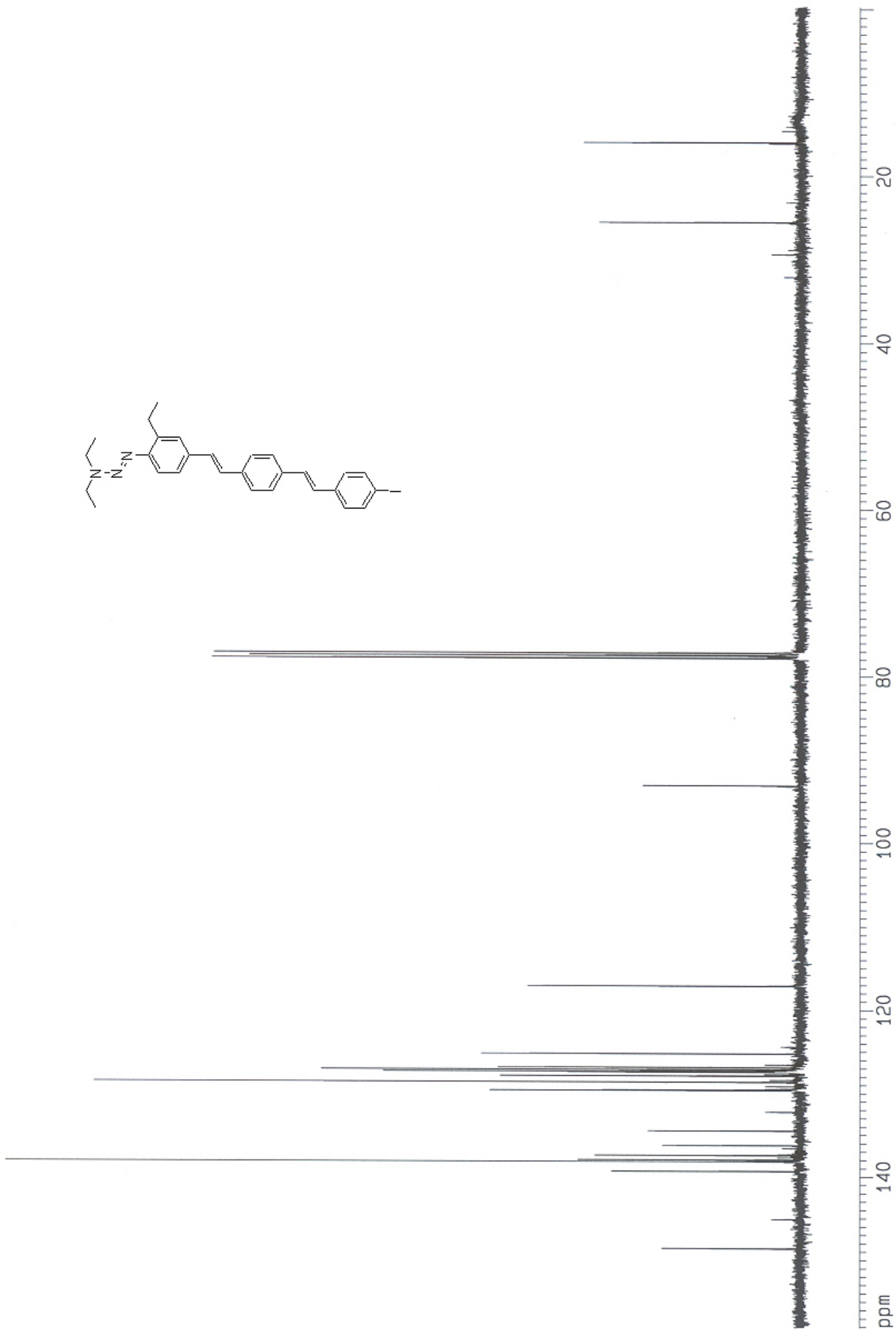

$981^{\circ} L L$ $\varepsilon 0 G^{\circ} \angle L$ Iट8 $\angle L$

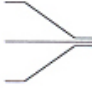

Gโ

$\angle O I^{\circ} \angle I T$

$\checkmark \mathrm{IJ}$.

698 . 951

$0 S I^{\prime} \angle Z I-$

$86 \varepsilon^{\circ} \angle 2 I-$

ट $\angle \nabla^{\circ} \angle C \downarrow$

$906^{\circ} \angle \mathcal{L}$

$979 \cdot 82 \tau$

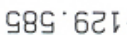

$999^{\circ} 62$ ᄃ

$\varepsilon\left\lceil G^{\circ} \nabla \varepsilon \downarrow\right.$

GEट ' $9 \varepsilon$

$\nabla ट \nabla \cdot \angle E D$

$966^{\circ} \angle \varepsilon \downarrow$

$\checkmark 6 I^{\circ} 8 \varepsilon$ - -

५॰E. $6 \varepsilon \downarrow$

$0 ट 9 \cdot 8 \nabla \downarrow$

udd

Figure S107: ${ }^{13} \mathrm{C}$ NMR of $\mathbf{4 7}$ 


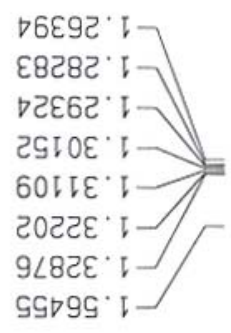

92868. ट $90 \angle 16.2-$

$0 \varepsilon \nabla L L^{\circ} \varepsilon$ टाट6 $L^{\circ} \varepsilon>$ $56608^{\circ} \varepsilon$ $\nabla 8 \angle 28^{\circ} \varepsilon$

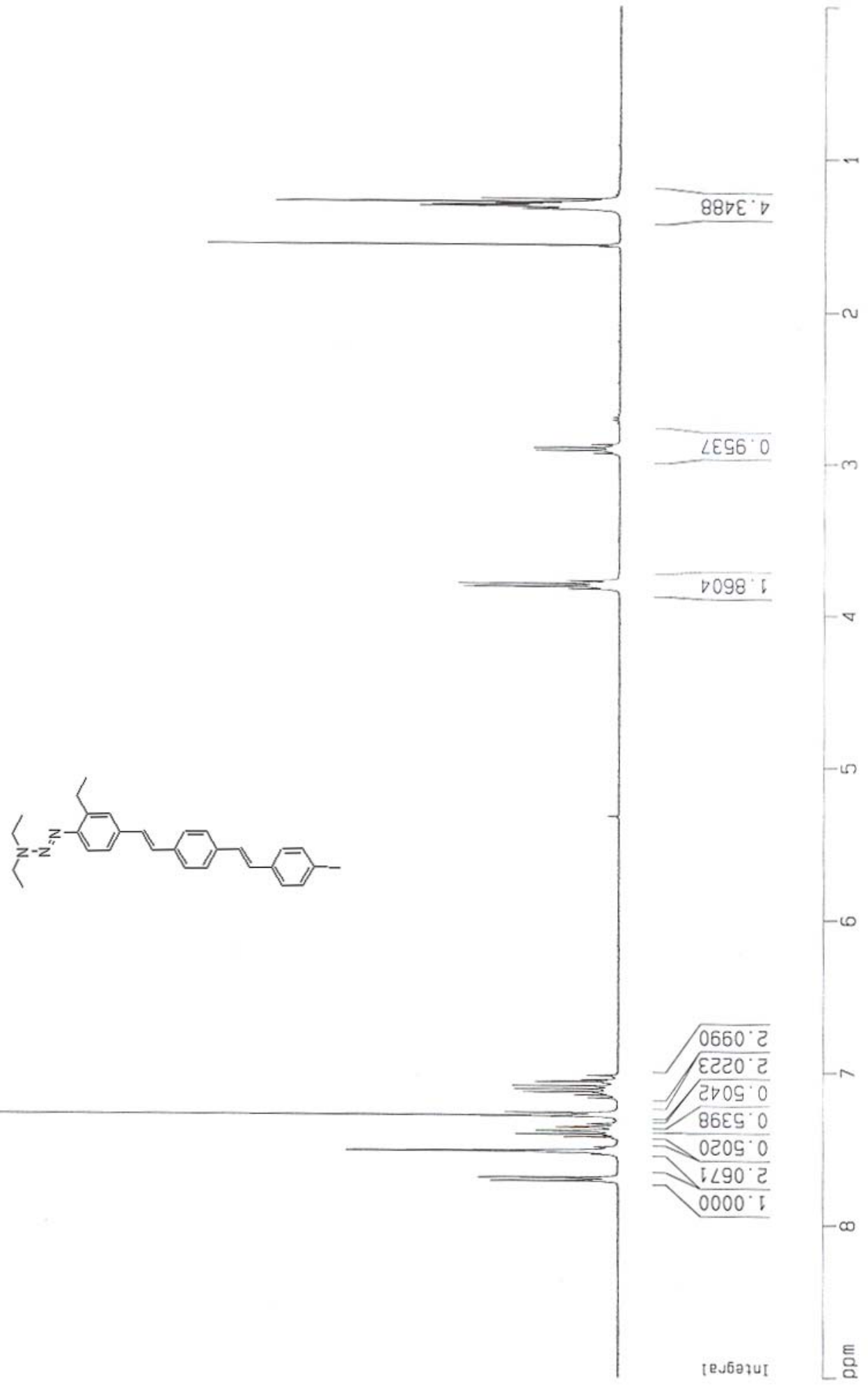

Figure S108: ${ }^{1} \mathrm{H}$ NMR of 47 


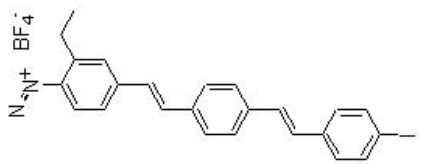

$628^{\circ} \varepsilon I$

619. 92

8ह8.6E

$500^{\circ}$ OD

टLI OD

6हE. O०

9OS. OD

$\varepsilon \angle 9^{\circ} \circ D$

$0 \rightarrow 8 \cdot 07$

โ8L'จ6

081 计

$\nabla \angle 9 \cdot 925$

GSI $\angle 2 I-$

$\nabla ट ा \cdot 85$ Г

$\varepsilon 8{ }^{\prime} 8$ I $^{\circ}$

$\angle 6 I^{\circ} 62 I$

દદ૪ 625 -

E9S 621

द8व.6टा

$98 I^{\circ} D E V$

966 GE

โ $9 \varepsilon^{\circ} \angle \varepsilon$ ᄃ

$90 \mathrm{r} \cdot 8 \varepsilon \mathrm{t}$

$\nabla 08^{\circ} 8 \varepsilon$

$\angle O E^{\circ} \sigma \varepsilon \downarrow$

$696^{\circ} 6 \nabla 1-$

6IS.OSI-

wdd

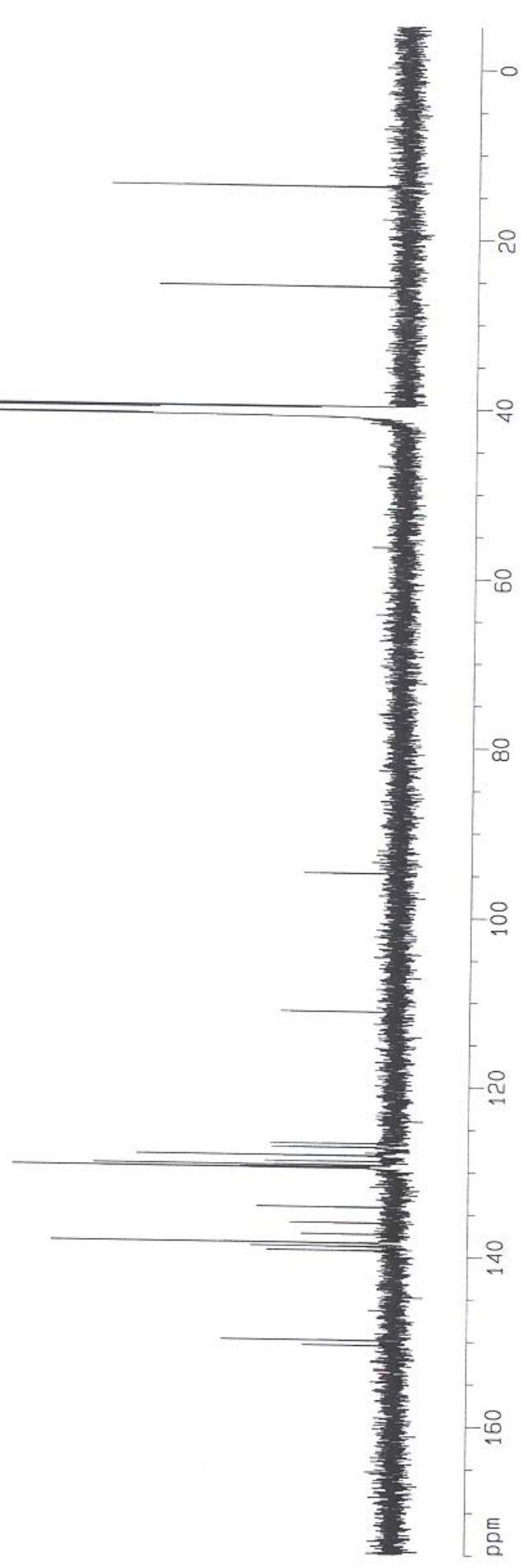

Figure S109: ${ }^{13} \mathrm{C}$ NMR of $\mathbf{4 8}$ 


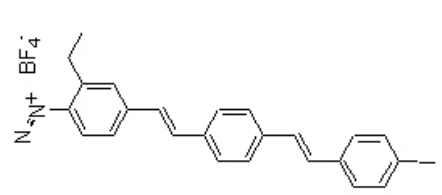

$\nabla I L \angle E^{\prime} \downarrow$

I I $98 \varepsilon^{\circ}$.

9LIOD.

I60'酒

DODIG

$\nabla \nabla \nabla \varepsilon 0^{\circ} \varepsilon$

9в6॰0 $\varepsilon-$

ᄂEจ90 $\varepsilon$

โโ6 $\angle 0^{\circ} \varepsilon-$

$6 \nabla G 29^{\circ} \varepsilon$

GIEGE $\angle$

GOटౌण $\angle-$

6৮89 $\nabla^{\circ} L$

$\checkmark 6 \triangleright \varepsilon G^{\circ} \angle-$

$89 \angle 99^{\circ} L$

$\varepsilon 8 \varepsilon\left[L^{\circ} \angle\right]$

I $\square C 2 L^{\circ} \angle$

G909L' $L$

969G $L$ ' $L$

$9 \angle 2 \angle L^{\circ}$

I $\triangle 6 E 8^{\circ} L$

$\angle 02 \angle 8^{\circ} \angle$

$98900 \cdot 8$

टЕ॰ट0 ० 8-

69E90.8-

$00009^{\circ} 8$

I $\forall<19.8$

wdd

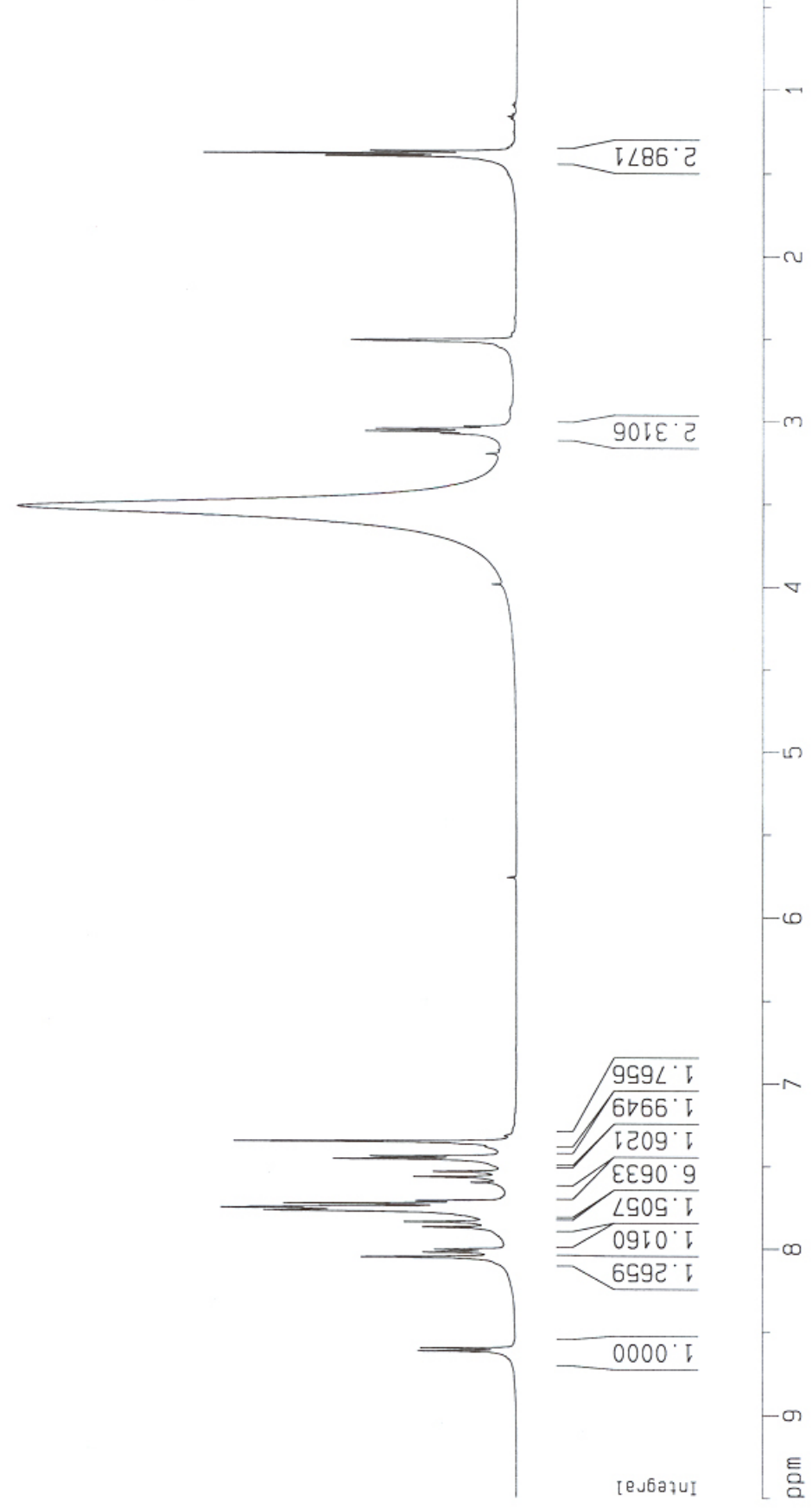

Figure S110: ${ }^{1} \mathrm{H}$ NMR of $\mathbf{4 8}$ 


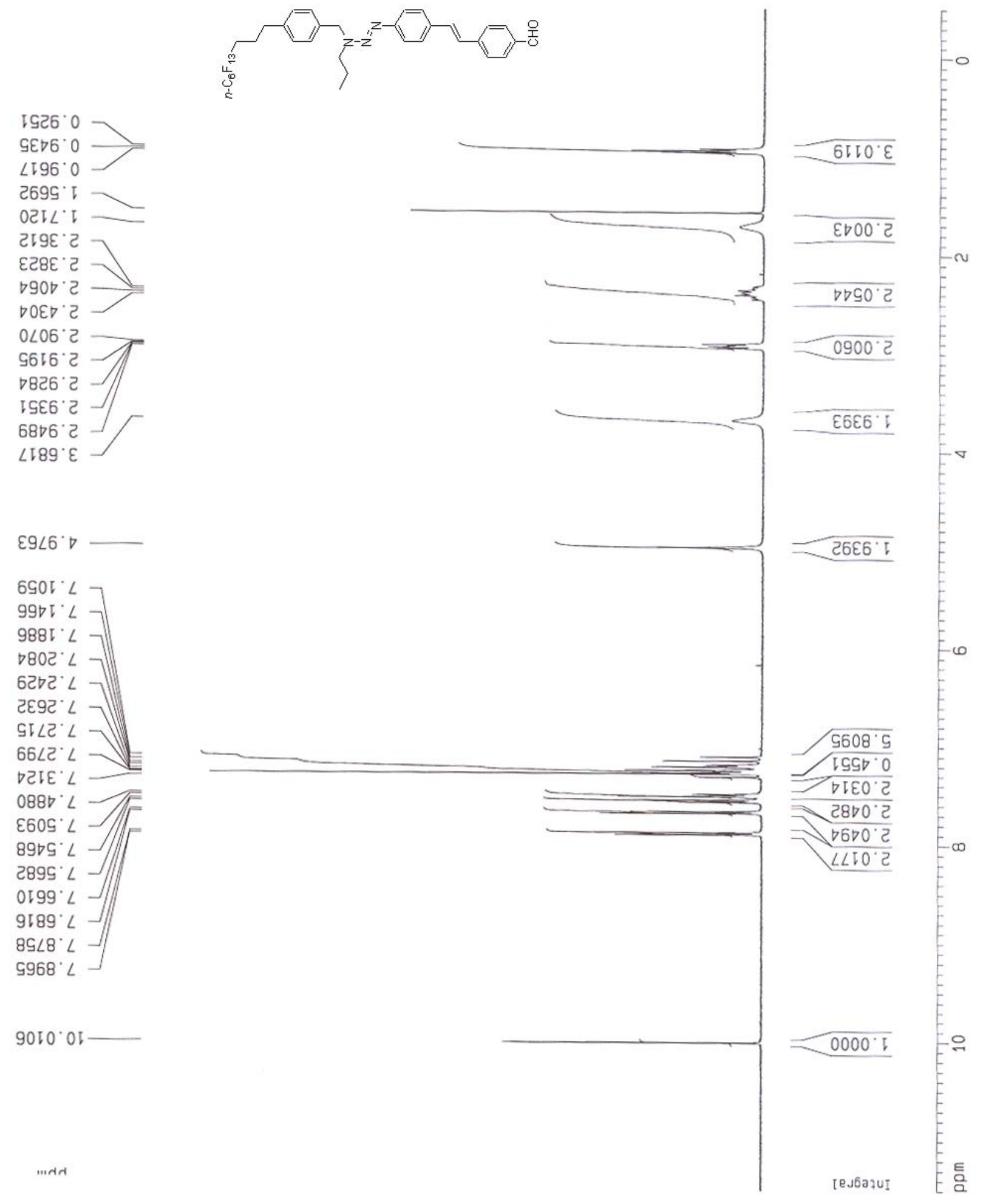

Figure S111: ${ }^{1} \mathrm{H}$ NMR of 49b 
$29 \cdot 951$

$\nabla 9 \cdot 9 \mathrm{t}$

$\varepsilon 9 \cdot 9 \mathrm{I}$

I9. 95 Г-

I0. $\nabla$ टा -

$\angle \varepsilon^{\cdot} \varepsilon ट \downarrow-$

OD.ટटा-

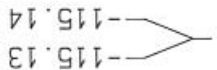

82 เ $18-$ 92 เ 吹 โ8-

wdd

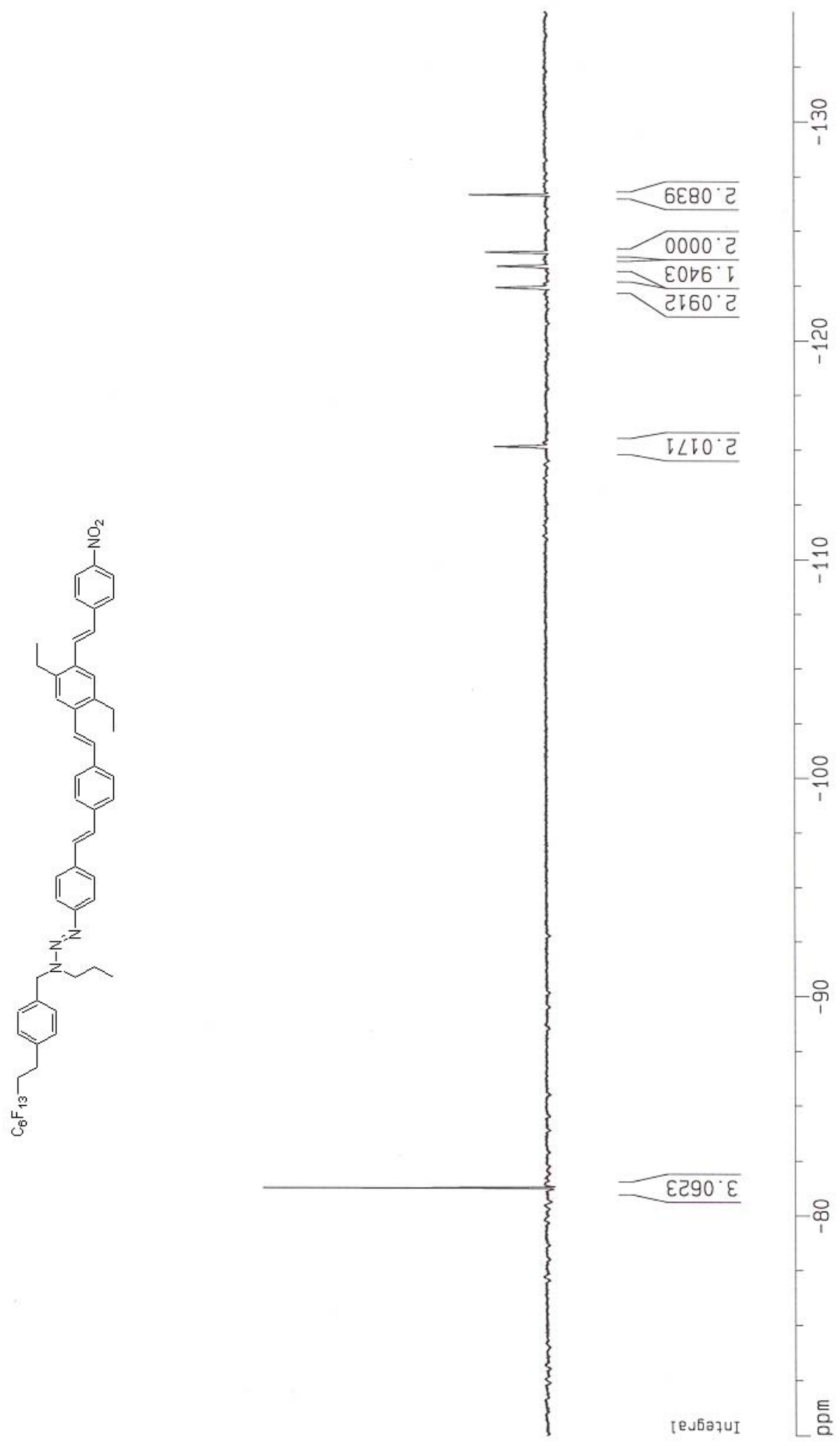

Figure S112: ${ }^{19} \mathrm{~F}$ NMR of $\mathbf{5 1 b}$ 


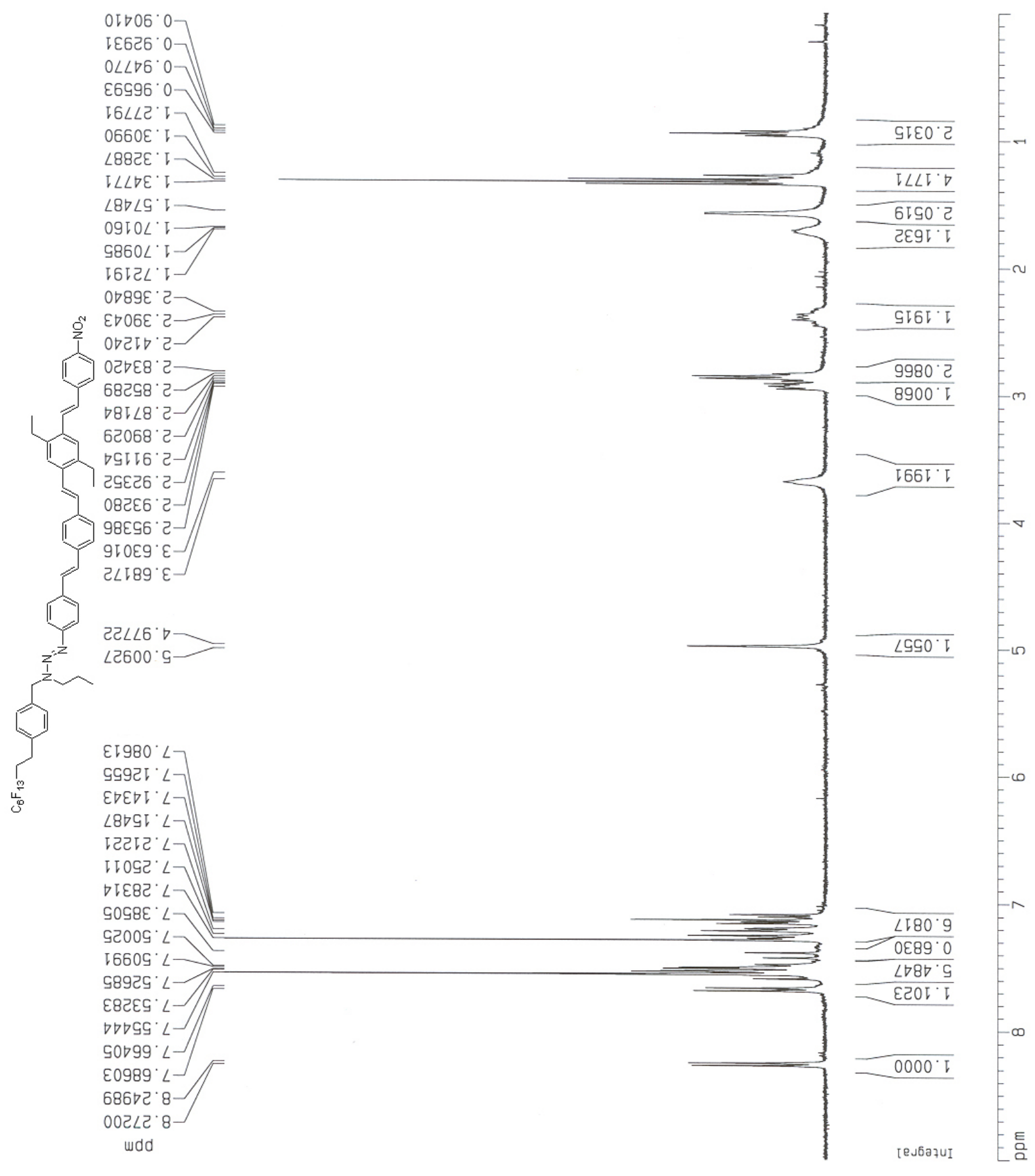

Figure S113: ${ }^{1} \mathrm{H}$ NMR of $\mathbf{5 1 b}$ 

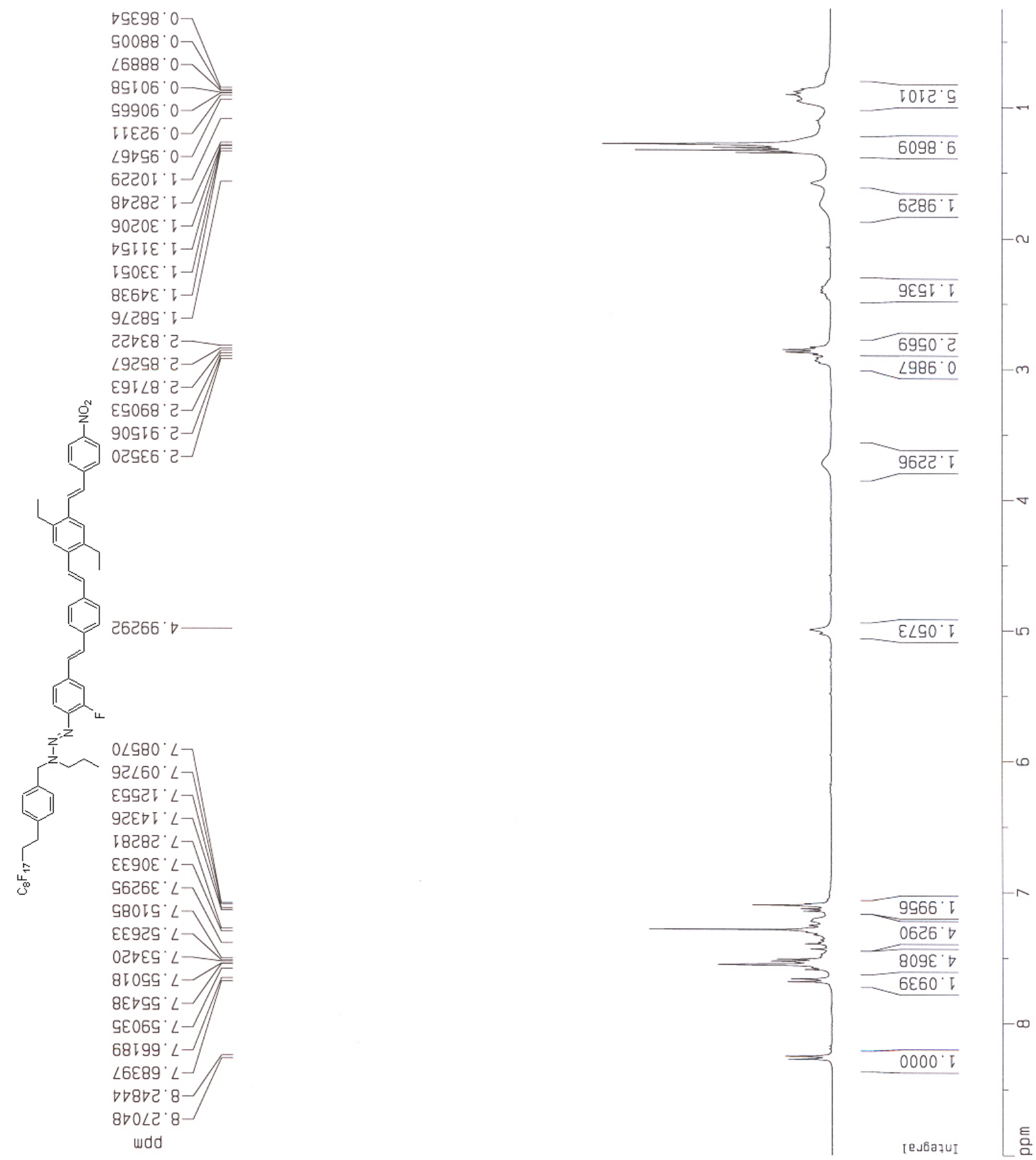

Figure S114: ${ }^{1} \mathrm{H}$ NMR of 51c 

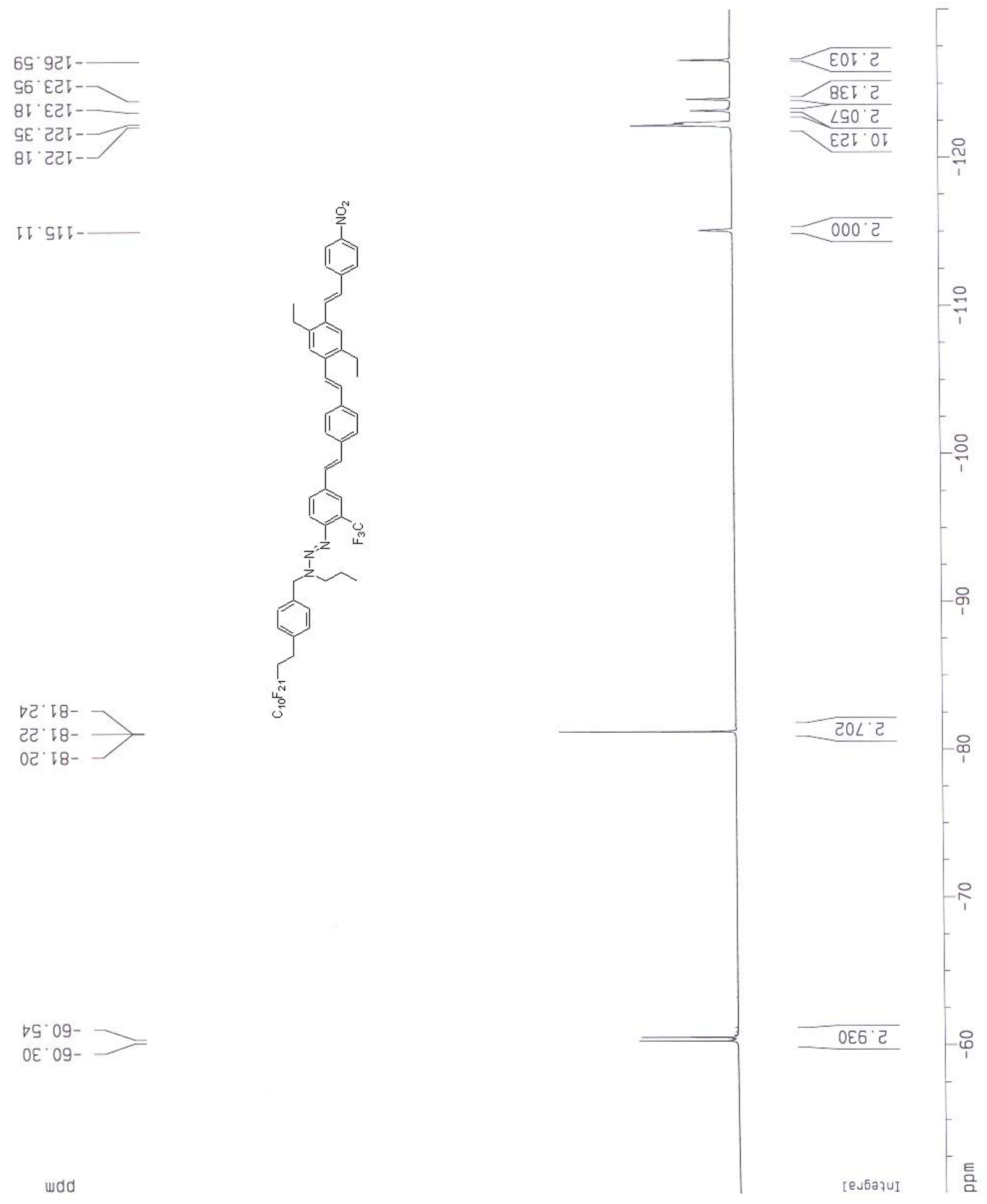

$\nabla S^{\circ} 09-\longrightarrow$

OE'09-

wdd

Figure S115: ${ }^{19} \mathrm{~F}$ NMR of 51d 




Figure S116: ${ }^{1} \mathrm{H}$ NMR of 51d 


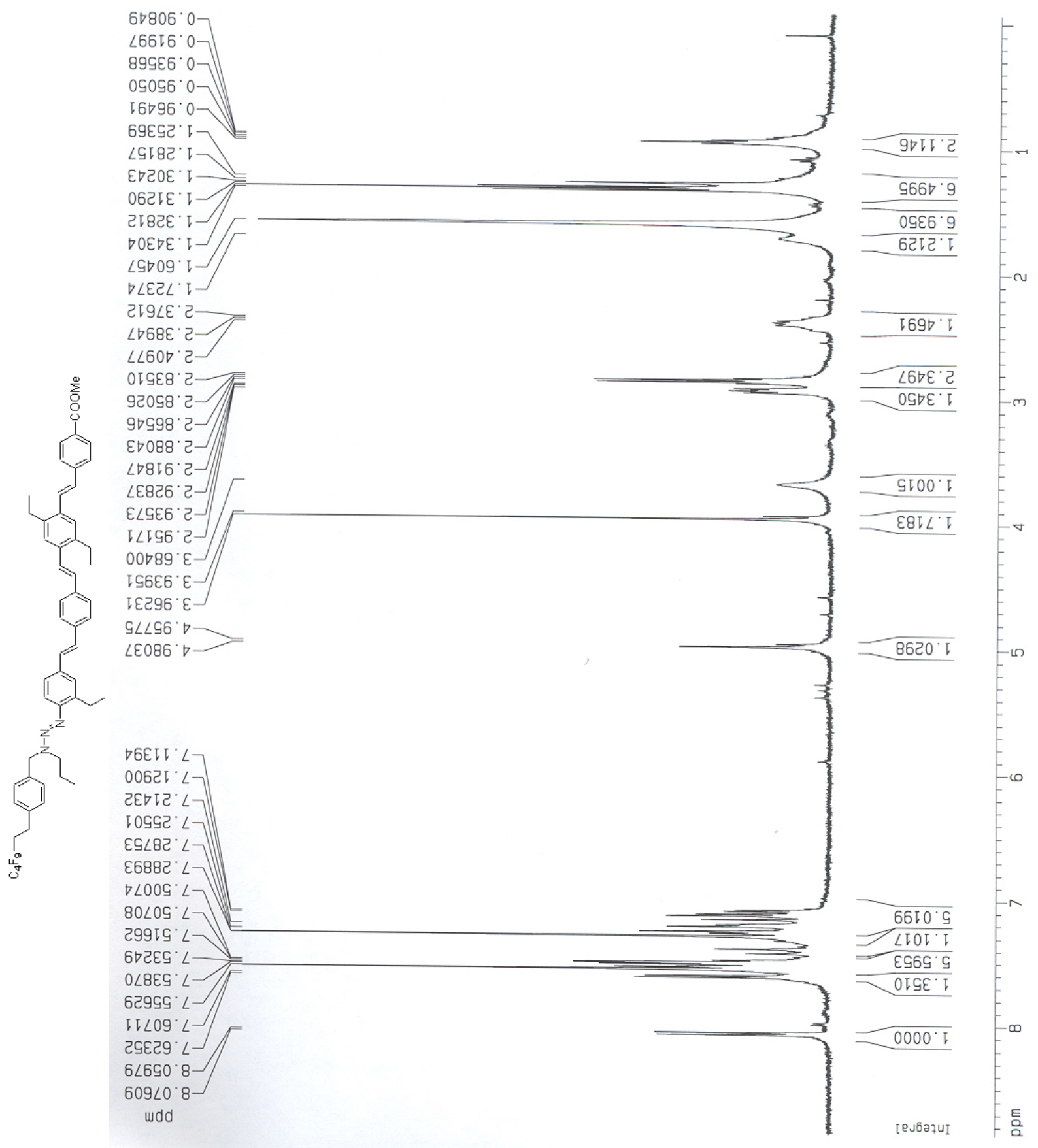

Figure S117: ${ }^{1} \mathrm{H}$ NMR of 52a 


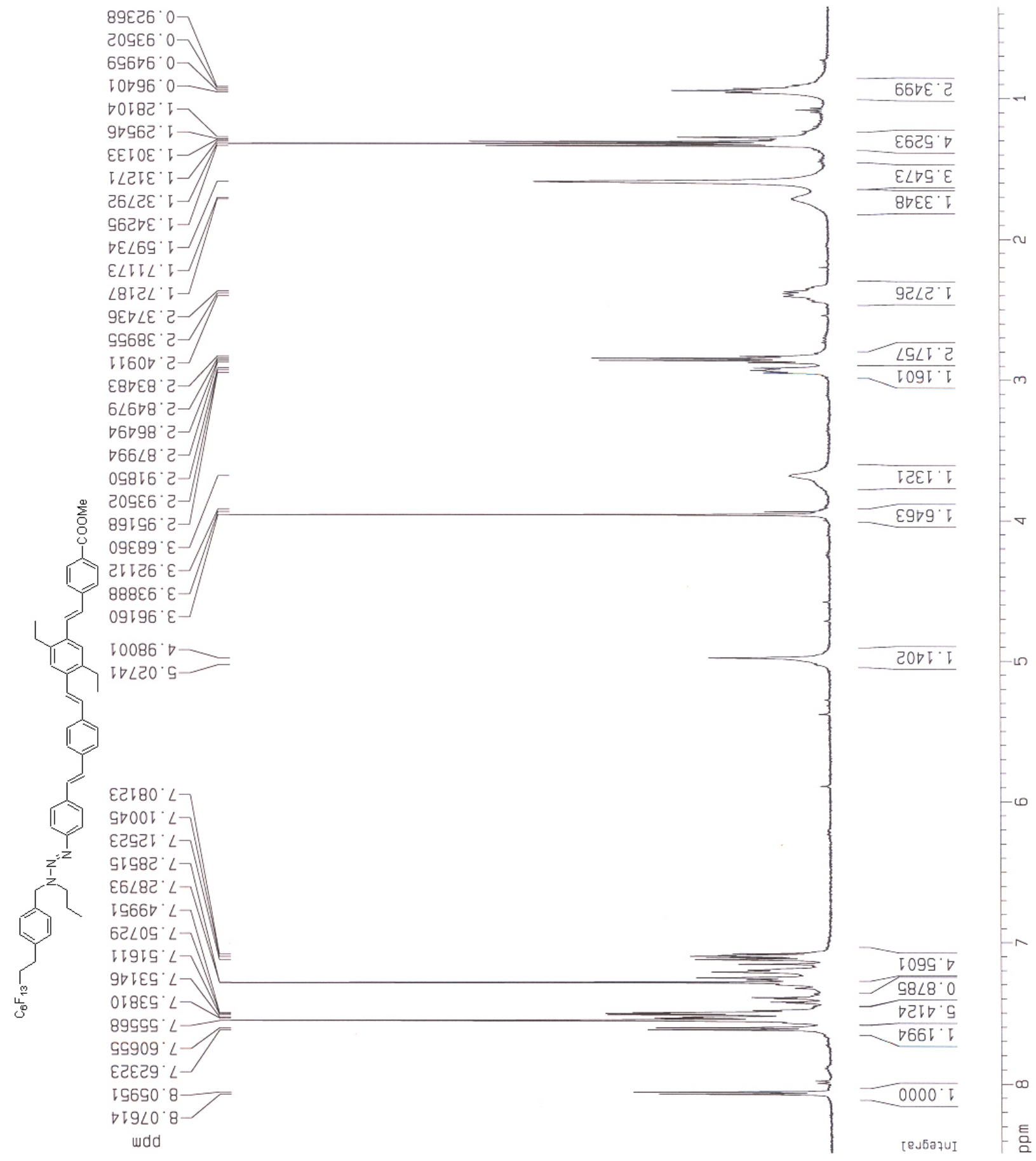

Figure S118: ${ }^{1} \mathrm{H}$ NMR of $\mathbf{5 2 b}$ 

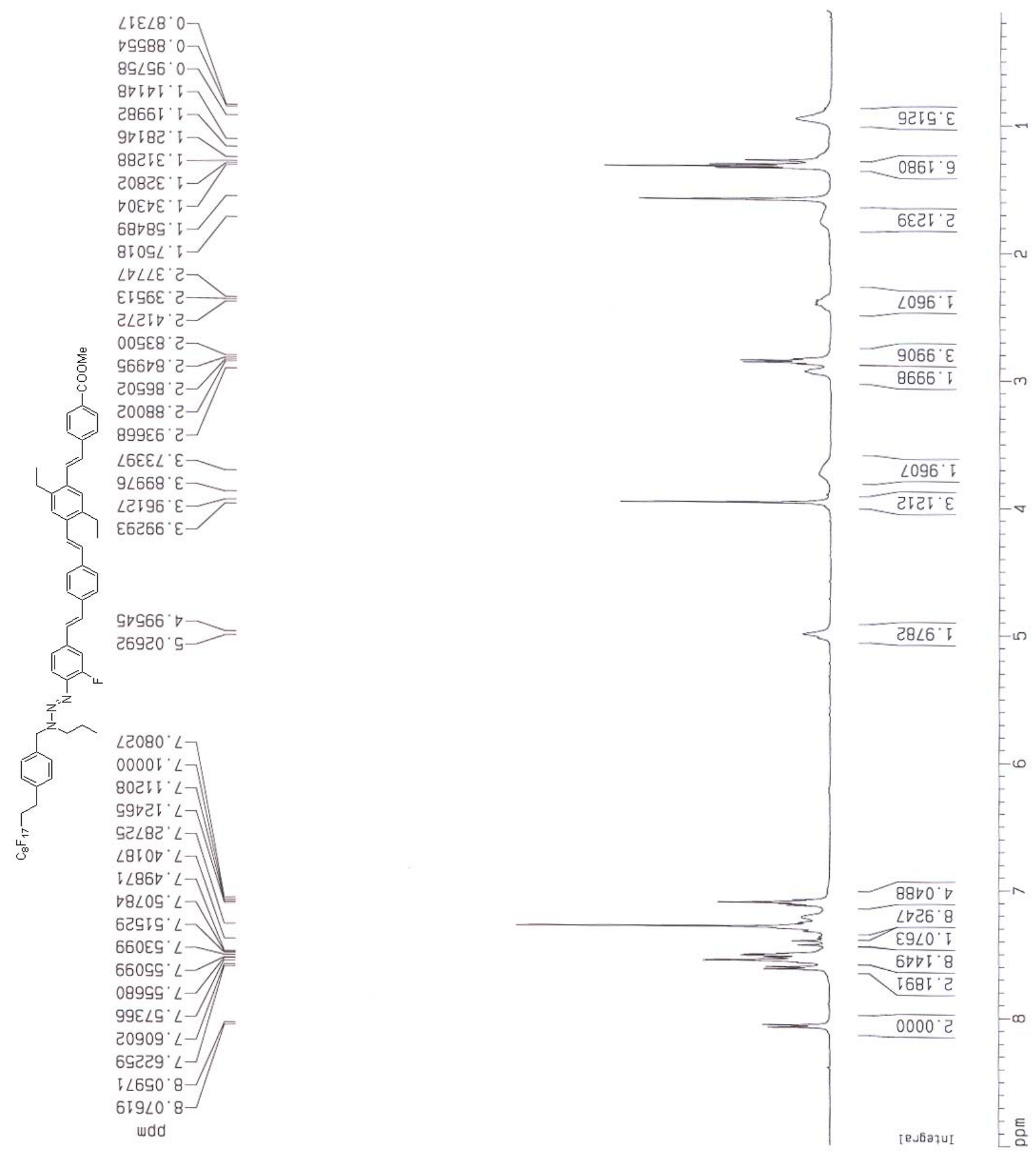

Figure S119: ${ }^{1} \mathrm{H}$ NMR of 52c 

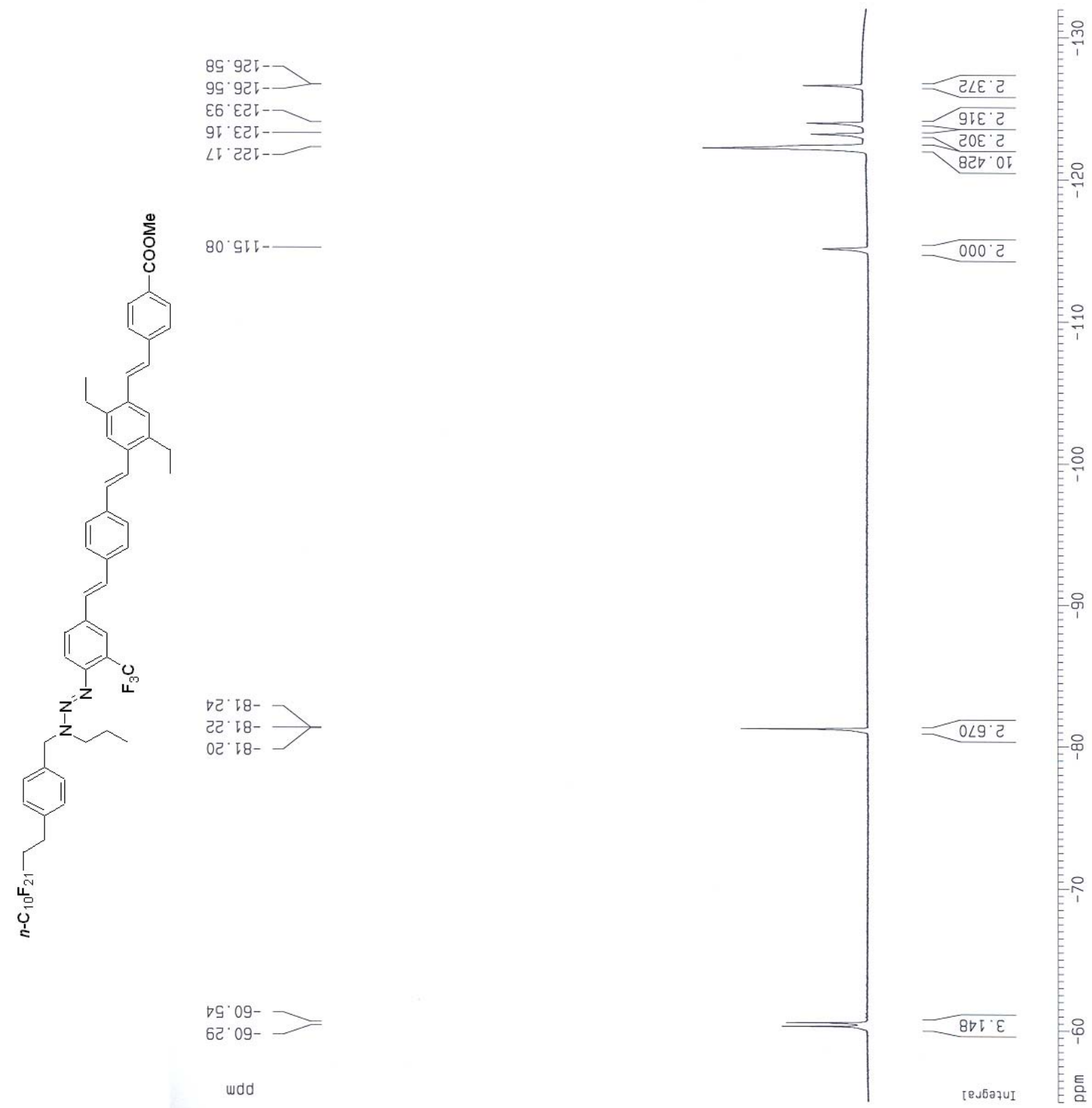

Figure S120: ${ }^{19}$ F NMR of $\mathbf{5 2 d}$ 

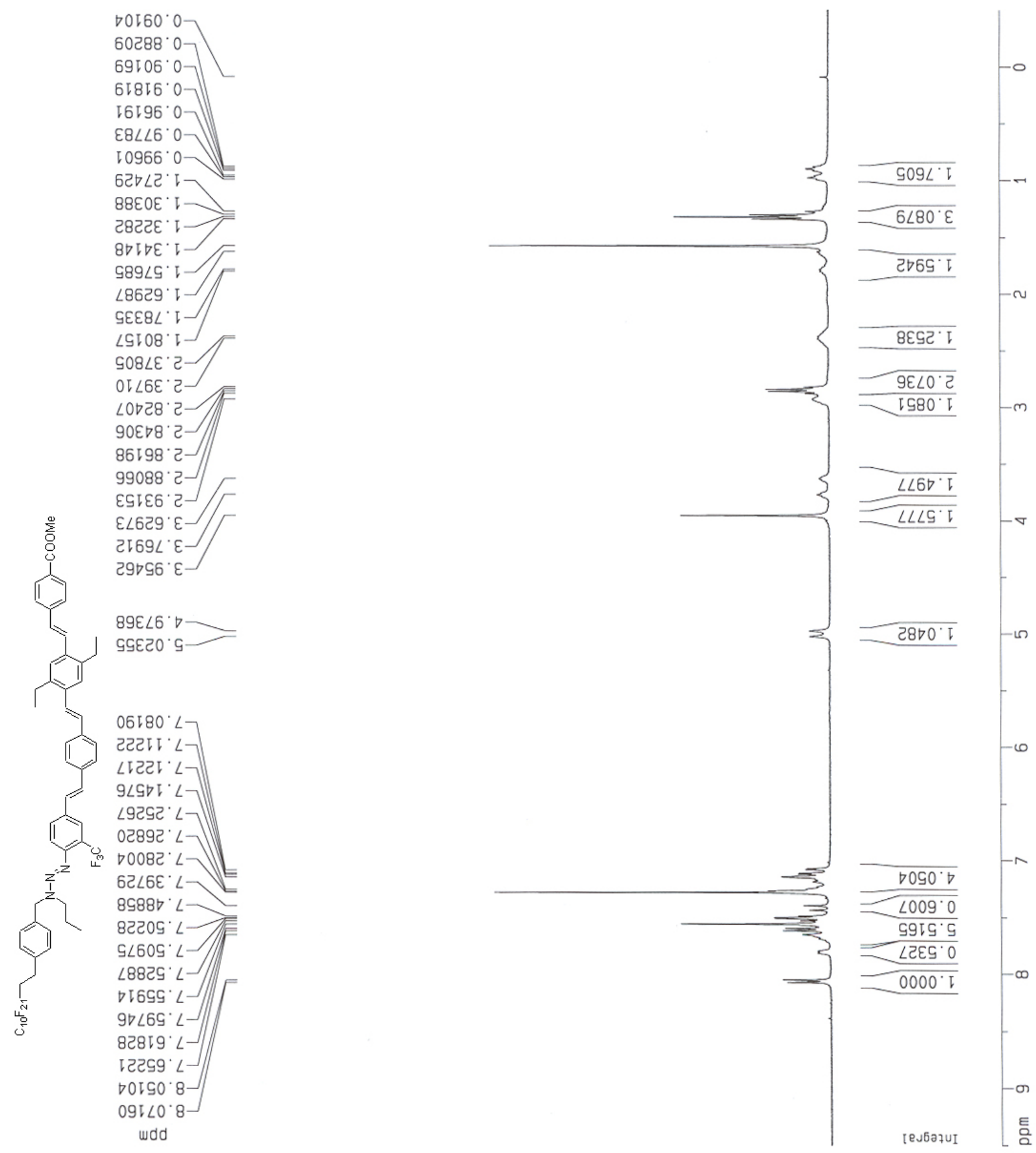

Figure S121: ${ }^{1} \mathrm{H}$ NMR of $\mathbf{5 2 d}$ 

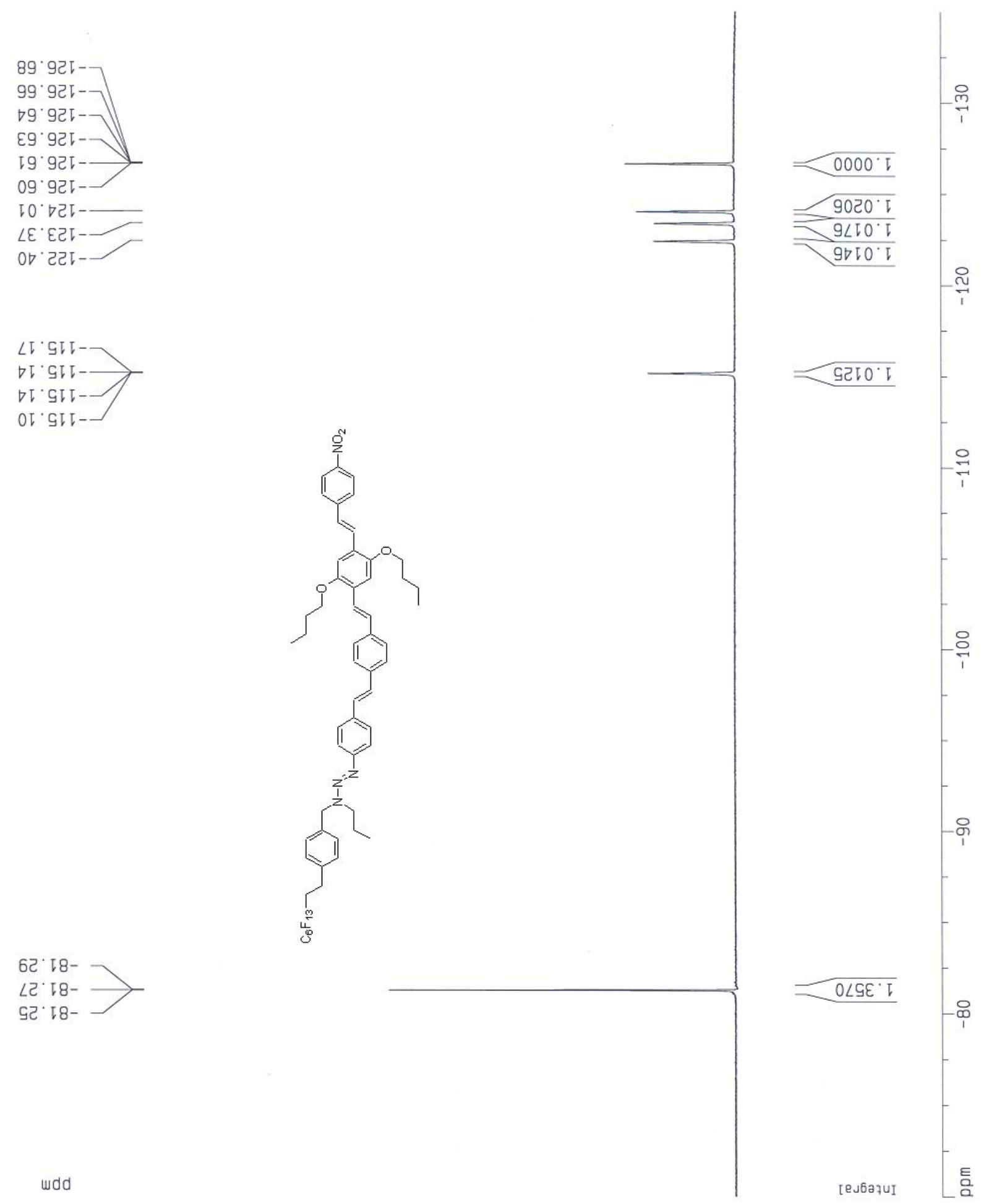

Figure S122: ${ }^{19} \mathrm{~F}$ NMR of $\mathbf{5 4 b}$ 

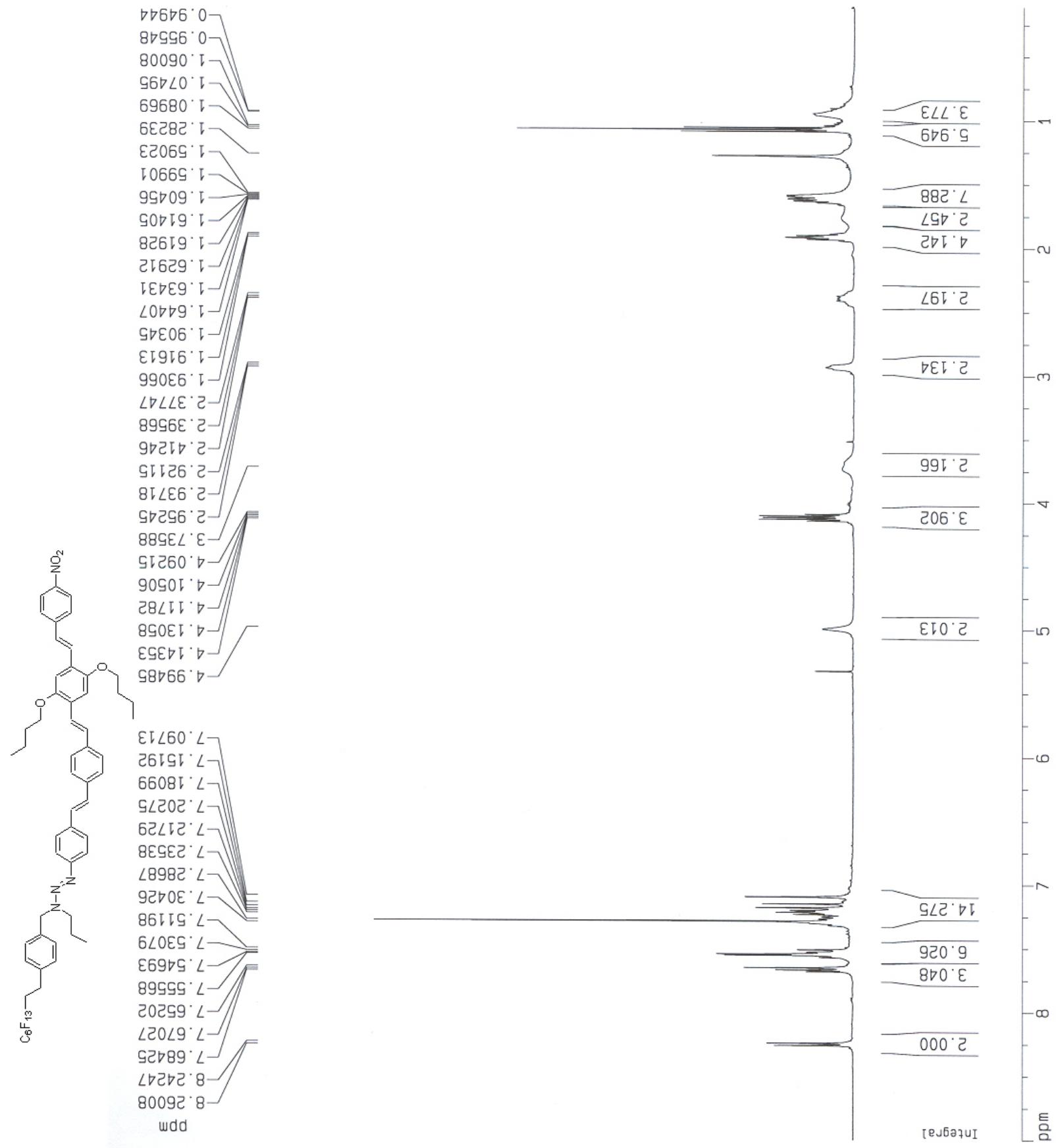

Figure S123: ${ }^{1}$ H NMR of $\mathbf{5 4 b}$ 
OE. $825-$

I 8 '

I9.9टा -

69.9टा-

99.9टा-

૬6. દટ -

0ટ 'દટ -

$O D \cdot 551-7$

9ा टटा--

ปเ SL-

wdd

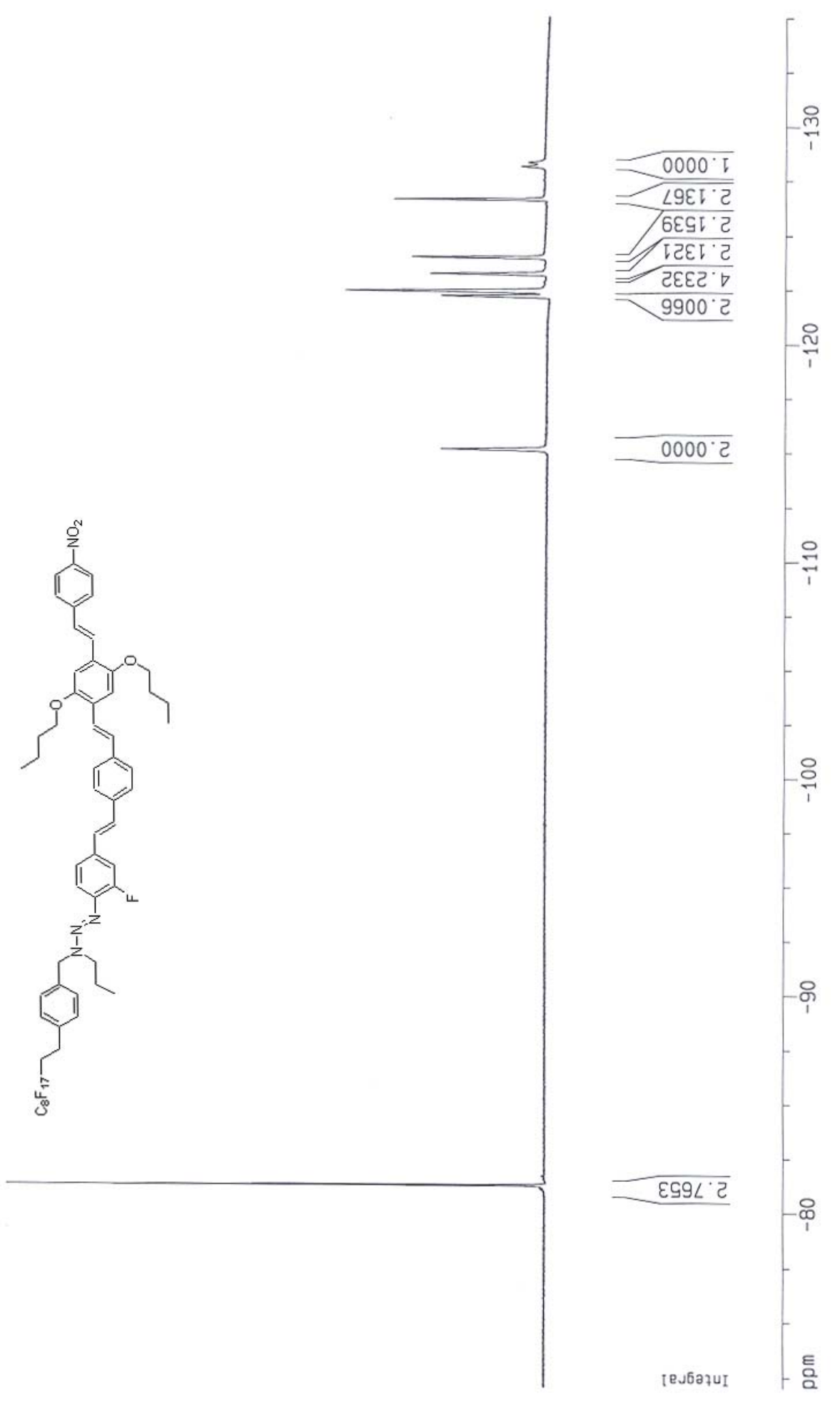

Figure S124: ${ }^{19} \mathrm{~F}$ NMR of $\mathbf{5 4 c}$ 


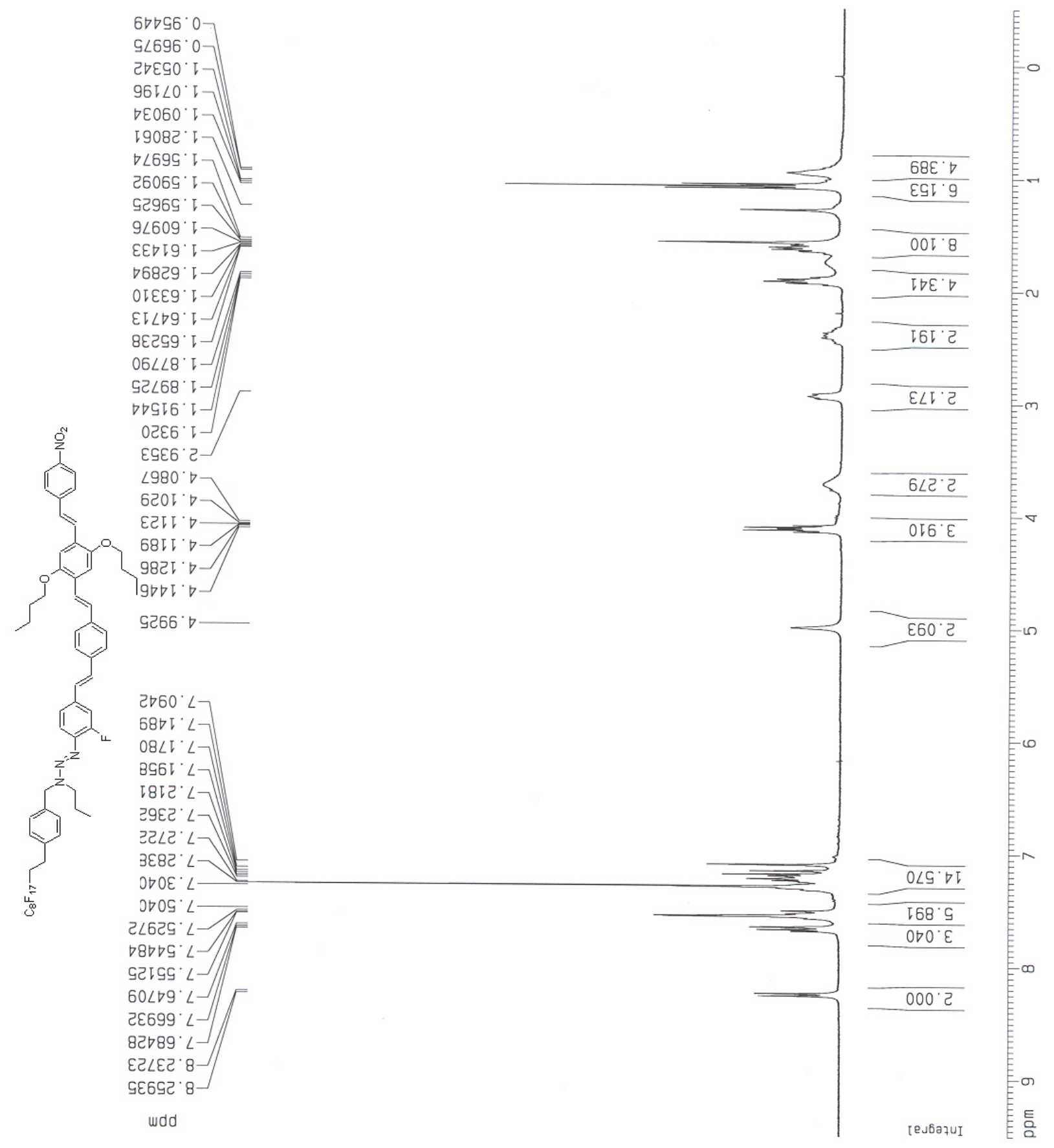

Figure S125: ${ }^{1} \mathrm{H}$ NMR of $\mathbf{5 4 c}$ 

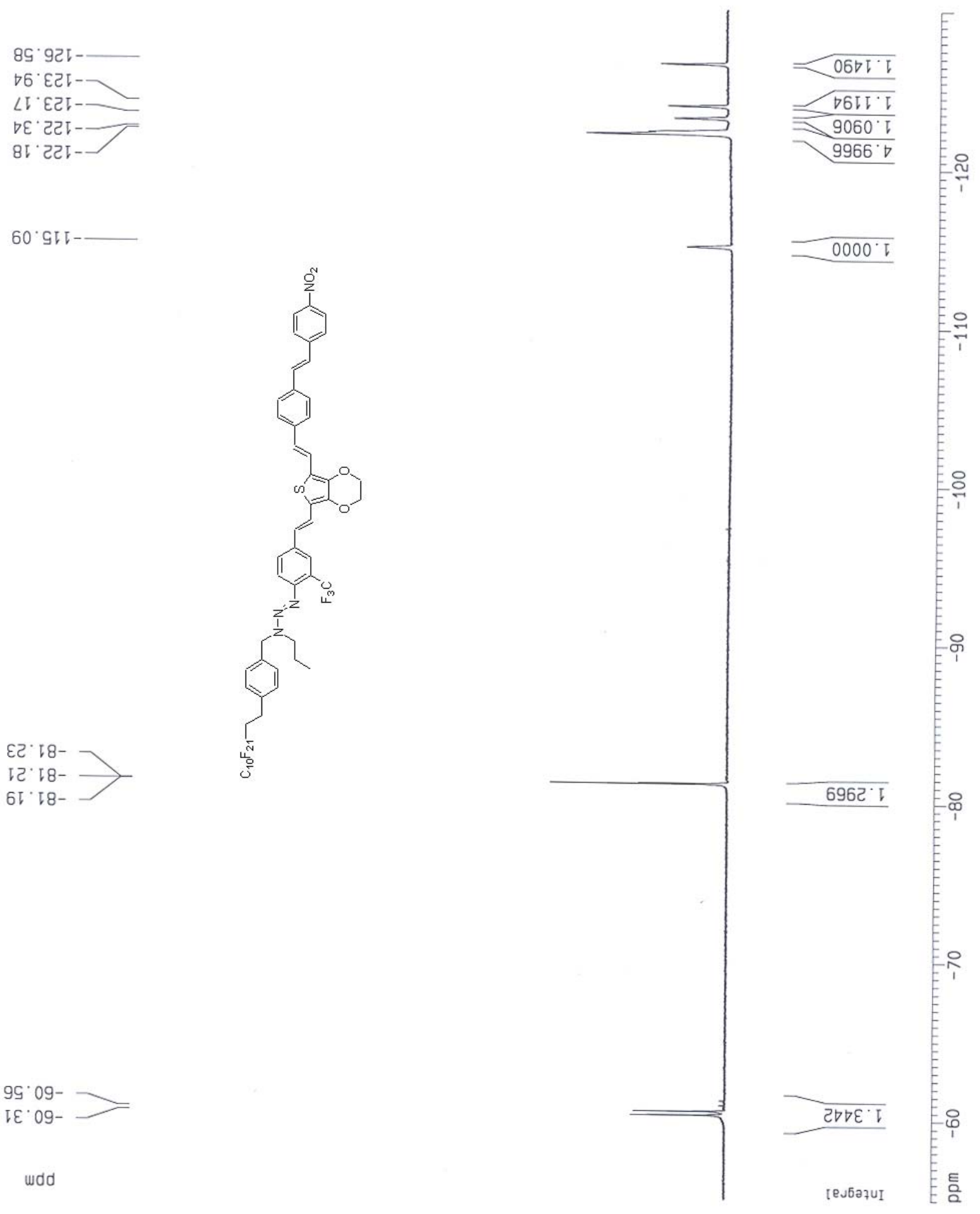

Figure S126: ${ }^{19}$ F NMR of 59d 


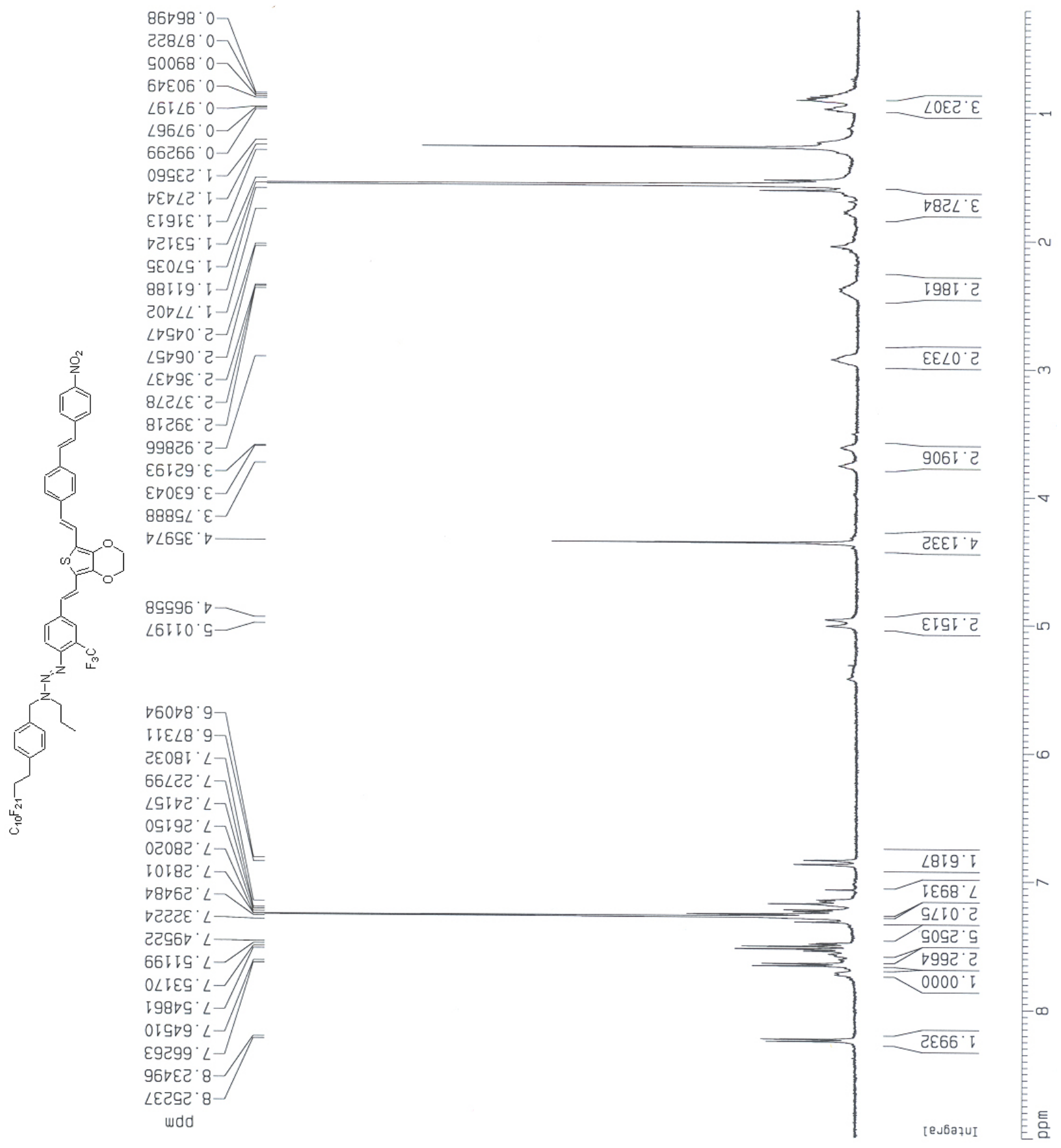

Figure S127: ${ }^{1} \mathrm{H}$ NMR of 59d 

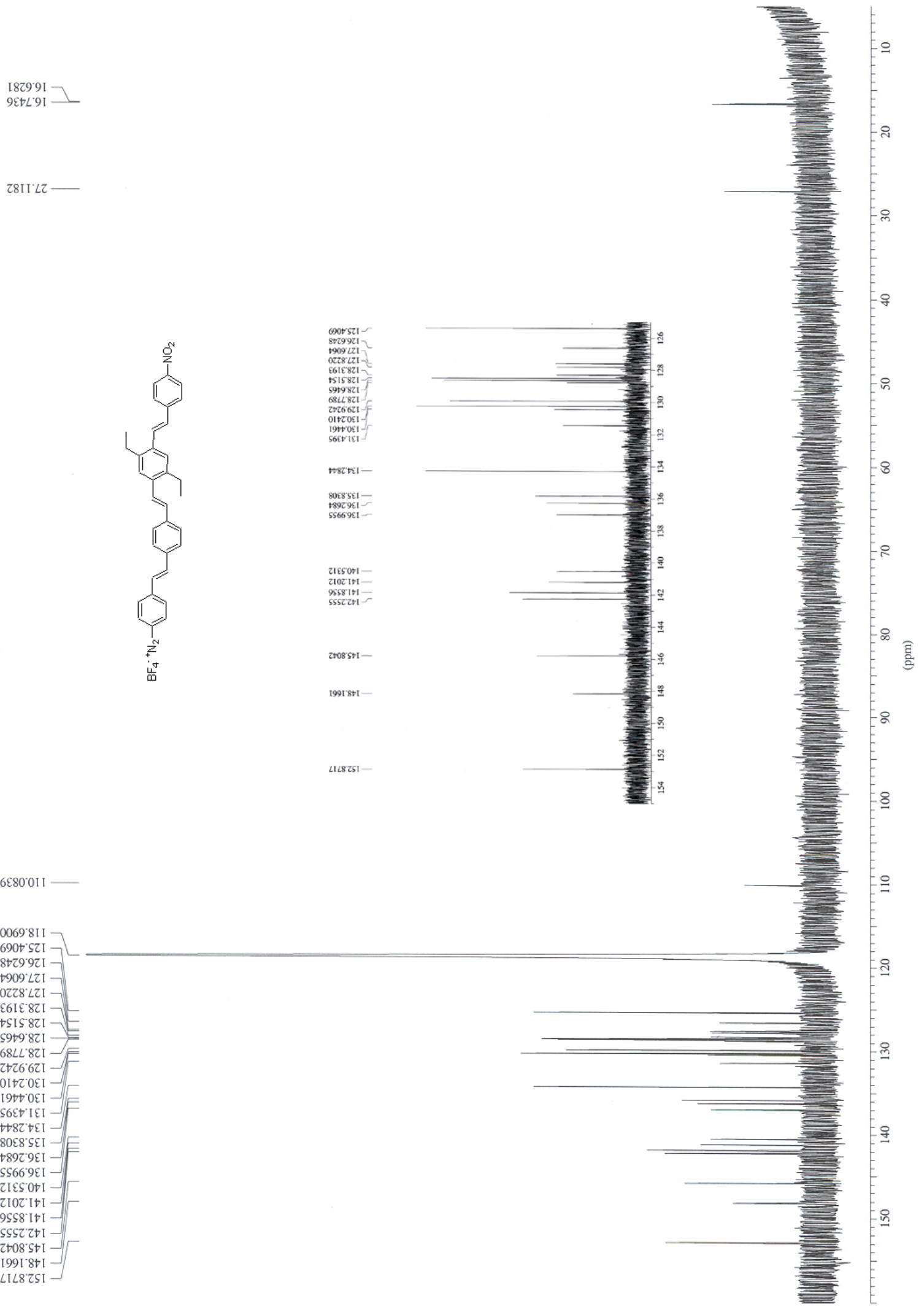

6£8001I -

Figure S128: ${ }^{13} \mathrm{C}$ NMR of $\mathbf{6 2 b}$ 

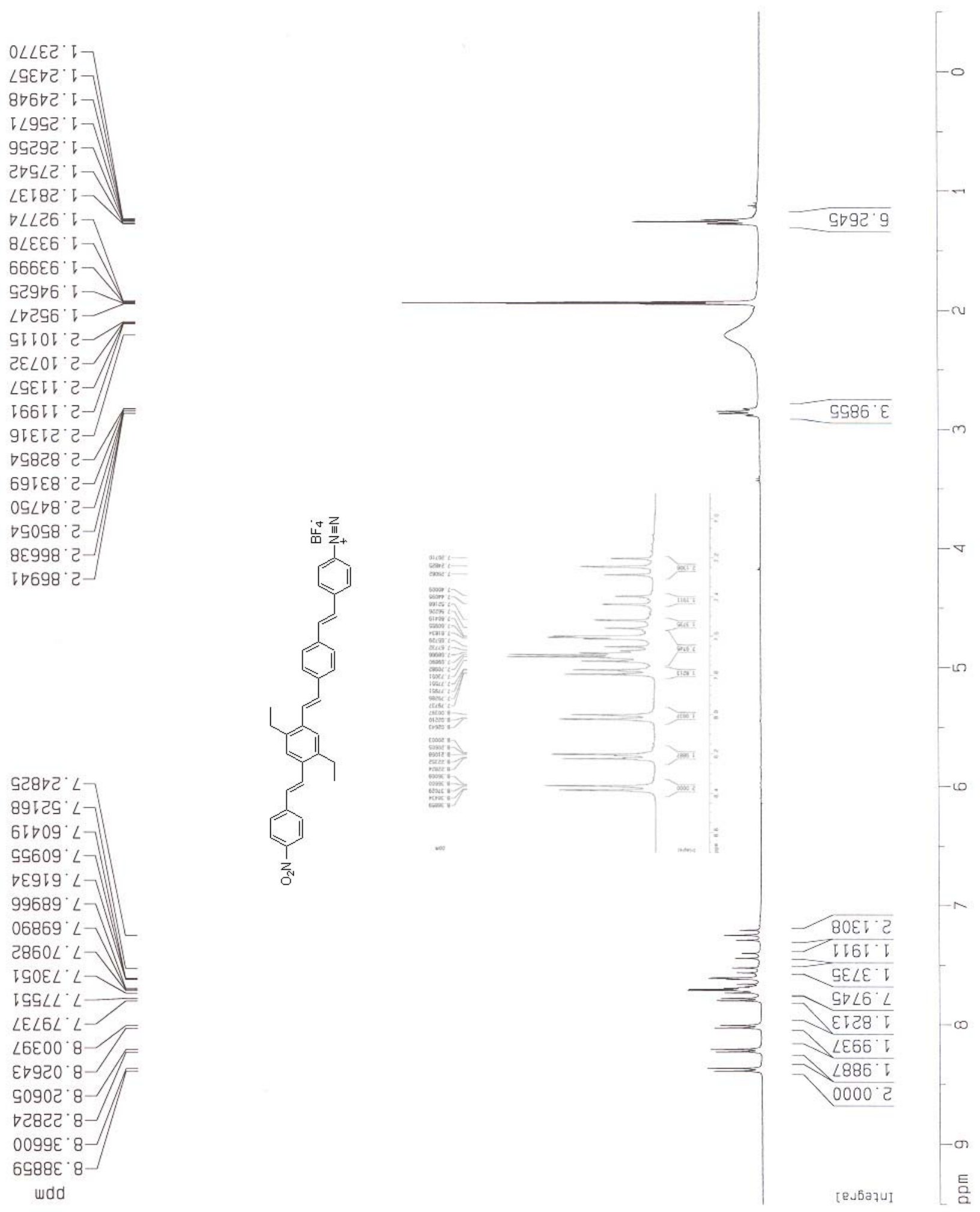

Figure S129: ${ }^{1} \mathrm{H}$ NMR of $\mathbf{6 2 b}$ 